\title{
On Faltings' method of almost étale extensions
}

\author{
Martin C. Olsson
}

\section{Contents}

1. Introduction 1

$\begin{array}{lll}2 . & \text { Almost mathematics and the purity theorem } & 10\end{array}$

3. Galois cohomology 15

\begin{tabular}{lll}
\hline 4. & Logarithmic geometry & 27
\end{tabular}

5. Coverings by $K(\pi, 1)$ 's 30

6. The topos $\mathscr{X}_{\mathrm{K}}^{\circ}$

7. Computing compactly supported cohomology using Galois cohomology 44

8. Proof of $\mid 6.16$. 54

9. $\quad$ The topos $\widehat{\mathscr{X}_{\bar{K}}} \quad 87$

10. An aside on Galois cohomology $\quad 89$

11. Fontaine's big rings 91

\begin{tabular}{lll}
\hline 12. & More computations of cohomology & 102
\end{tabular}

13. Crystalline cohomology and crystalline sheaves 106

14. Proof of the comparison theorem 113

$\begin{array}{lll}15 . & \text { From } \mathrm{B}_{\text {cris }}(\bar{V}) \text { to } \mathrm{B}_{\text {cris }}(\bar{V}) & 118\end{array}$

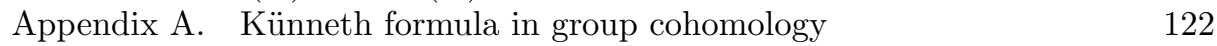

$\begin{array}{ll}\text { References } & 124\end{array}$

\section{Introduction}

1.1. The last decade has been a time of tremendous activity in $p$-adic Hodge Theory. We do not intend to give a complete account of these new developments here, but let us at least mention the surveys 2 providing an overview of recent topics in the theory of $p$-adic representations, the survey of $p$-adic cohomology [34, the survey of $p$-adic Hodge theory for torsion coefficients [8], Tsuji's survey of his proof of the $C_{\mathrm{st}}$-conjecture of Fontaine [51, and for an introduction to the main ideas of $p$-adic Hodge theory the beautiful article [27. We should also note that in the last decade there has been an increasing interest in non-abelian $p$-adic Hodge theory and its relationship to motives [20, [35, [41, [52.

Instead of attempting the impossible task of trying to cover all of these topics in one article we will instead discuss one aspect of the theory: Faltings' method of 
almost étale extensions. Faltings' method is one of the most powerful tools in $p$ adic Hodge theory and yields in particular a proof of the $C_{\text {st }}$-conjecture of Fontaine which was first proven by Tsuji in 49 using syntomic cohomology. It is also to the author's knowledge the best technology currently available to deal with coefficients and cohomology with compact support of open varieties. Unfortunately for various reasons (not the least of which is the very technical nature of Faltings' theory), the approach to $p$-adic Hodge theory using "almost mathematics" does not seem to be so widely studied.

1.2. Let us begin by reviewing the main result of $p$-adic Hodge theory in the simplest situation of good reduction, and the various approaches to the theory in this context.

Let $p$ be a prime, and let $V$ be a complete discrete valuation ring of mixed characteristic $(0, p)$ and perfect residue field $k$. Let $K$ be the fraction field of $V$, and fix an algebraic closure $K \hookrightarrow \bar{K}$. Let $W \subset V$ be the ring of Witt vectors of $k$, and let $K_{0} \subset K$ be the field of fractions of $W$. The ring $W$ comes equipped with a lifting of Frobenius $\sigma: W \rightarrow W$ which also induces an automorphism of $K_{0}$ which we denote by the same letter.

Let $X / V$ be a smooth proper scheme. The theory of $p$-adic Hodge theory concerns the comparison between the étale cohomology $H^{*}\left(X_{\bar{K}}, \mathbb{Q}_{p}\right)$ and the algebraic de-Rham cohomology $H_{\mathrm{dR}}^{*}\left(X_{K}\right)$ of the generic fiber.

To understand this comparison one must consider the additional structures that these cohomology groups are endowed with. The étale cohomology $H^{*}\left(X_{\bar{K}}, \mathbb{Q}_{p}\right)$ is a continuous $G_{K}:=\operatorname{Gal}(\bar{K} / K)$-representation, and the de Rham cohomology $H_{\mathrm{dR}}^{*}\left(X_{K}\right)$ comes equipped with the following data:

(i) A descending filtration $F$ · (the Hodge filtration).

(ii) A graded $K_{0}$-vector space $M_{0}$ with a $\sigma$-semi-linear automorphism $\varphi$ : $M_{0} \rightarrow M_{0}$ (Frobenius) and an isomorphism $K \otimes_{K_{0}} M_{0} \simeq H_{\mathrm{dR}}^{*}\left(X_{K}\right)$. The $K_{0}$-vector space $M_{0}$ is given by the crystalline cohomology of the closed fiber of $X$.

This package is formalized by defining a filtered $\varphi$-module to be a triple $\left(D, \varphi, \operatorname{Fil}_{D}\right)$ consisting of a finite dimensional $K_{0}$-vector space $D$ with a $\sigma$-semi-linear automorphism $\varphi: D \rightarrow D$ and a descending separated and exhaustive filtration Fil $_{D}$ on $D_{K}:=D \otimes_{K_{0}} K$.

To pass between the étale and de Rham cohomologies (with their additional structure) one uses Fontaine's ring $\mathrm{B}_{\text {cris }}(V)$ (see section 11). This is a $K_{0}$-algebra which comes equipped with a Frobenius automorphism $\varphi$ and an action of $G_{K}$. The ring $\mathrm{B}_{\text {cris }}(V)$ is a subring of a larger $K$-algebra $\mathrm{B}_{\mathrm{dR}}(V)$ which is a discrete valuation field. In particular, the valuation on $\mathrm{B}_{\mathrm{dR}}(V)$ defines a filtration on $\mathrm{B}_{\mathrm{dR}}(V)$. If $E$ is a filtered $K$-vector space then we view $E \otimes_{K} \mathrm{~B}_{\mathrm{dR}}(V)$ as filtered using the product filtration.

For a continuous $G_{K}$-representation $W$ define

$$
D(W):=\left(W \otimes_{\mathbb{Q}_{p}} \mathrm{~B}_{\text {cris }}(V)\right)^{G_{K}} .
$$

This is known to be a finite-dimensional $\mathbb{Q}_{p}$-vector space, and it has a natural structure of a filtered $\varphi$-module. Namely, it inherits a semi-linear Frobenius automorphism $\varphi_{D(W)}$ from the automorphism on $\mathrm{B}_{\text {cris }}(V)$, and there is a filtration $\mathrm{Fil}_{D(W)}$ on $D(W)_{K}:=D(W) \otimes_{K_{0}} K$ induced by the filtration on $D(W)_{K} \otimes_{K} \mathrm{~B}_{\mathrm{dR}}(V)$. 
The representation $W$ is called crystalline if the natural map (which is always injective)

$$
D(W) \otimes_{K_{0}} \mathrm{~B}_{\text {cris }}(V) \rightarrow W \otimes_{\mathbb{Q}_{p}} \mathrm{~B}_{\text {cris }}(V)
$$

is an isomorphism. In this case we say that $\left(D(W), \varphi_{D(W)}, \mathrm{Fil}_{D(W)}\right)$ is associated to $W$. If $W$ is crystalline one can recover $W$ from $\left(D(W), \varphi_{D(W)}, \operatorname{Fil}_{D(W)}\right)$ as

$$
\left\{x \in D(W) \otimes_{K_{0}} \mathrm{~B}_{\text {cris }}(V) \mid \varphi(x)=x, \quad x \otimes 1 \in \operatorname{Fil}^{0}\left(D(W)_{K} \otimes_{K} \mathrm{~B}_{\mathrm{dR}}(V)\right)\right\} .
$$

A filtered $\varphi$-module $\left(D, \varphi, \mathrm{Fil}_{D}\right)$ is called admissible if it is isomorphic to $\left(D(W), \varphi_{D(W)}, \operatorname{Fil}_{D(W)}\right)$ for some crystalline representation $W$.

The main result of $p$-adic Hodge theory in the present situation is the following:

Theorem $1.3([\mathbf{1 4}, \mathbf{1 8}, 49])$. The $G_{K}$-representation $H^{*}\left(X_{\bar{K}}, \mathbb{Q}_{p}\right)$ is crystalline and is associated to $H_{\mathrm{dR}}^{*}\left(X_{K}\right)$ with the additional structure (i) and (ii) above.

REMARK 1.4. In the present good reduction setting, theorem 1.3 was first proven by Fontaine and Messing in [18] under the assumption that $p>\operatorname{dim}\left(X_{K}\right)$ and $K_{0}=K$. Subsequently Kato and Messing were able to extend this result to the case when $\operatorname{dim}\left(X_{K}\right)<(p-1) / 2[\mathbf{3 1}, \mathbf{3 2}$. The general result was then proven by Tsuji and Faltings who also proved a comparison result without the assumption of good reduction (where the statement is more complicated).

REMARK 1.5. One application of 1.3 , from which the terminology ' $p$-adic Hodge theory' derives, is the following. As mentioned above the ring $\mathrm{B}_{\mathrm{dR}}(V)$ is a discrete valuation field, and its residue field is $\mathbb{C}_{p}$ (the $p$-adic completion of $\bar{K}$ ). Using this one shows that there is an isomorphism of graded rings with $G_{K}$-action (the subscript HT stands for Hodge-Tate)

$$
\mathrm{B}_{\mathrm{HT}}(V):=\operatorname{gr}^{*} \mathrm{~B}_{\mathrm{dR}}(V) \simeq \oplus_{i \in \mathbb{Z}} \mathbb{C}_{p}(i),
$$

where multiplication on the right is given by the natural isomorphisms $\mathbb{C}_{p}(i) \otimes$ $\mathbb{C}_{p}(j) \rightarrow \mathbb{C}_{p}(i+j)$. Now if $W$ is a crystalline representation, then the isomorphism 1.2.1 induces upon tensoring with $\mathrm{B}_{\mathrm{dR}}(V)$ and passing to the associated graded modules an isomorphism

$$
\operatorname{gr}^{*}\left(D(W)_{K}\right) \otimes \mathrm{B}_{\mathrm{HT}}(V) \simeq W \otimes_{\mathbb{Q}_{p}} \mathrm{~B}_{\mathrm{HT}} .
$$

In the case when $W=H^{m}\left(X_{\bar{K}}, \mathbb{Q}_{p}\right)$ the degree 0 part of this isomorphism yields a $G_{K}$-equivariant isomorphism

$$
H^{m}\left(X_{\bar{K}}, \mathbb{Q}_{p}\right) \otimes \mathbb{C}_{p} \simeq \oplus_{i+j=m} H^{i}\left(X_{K}, \Omega_{X_{K}}^{j}\right) \otimes_{K} \mathbb{C}_{p}(-j)
$$

called the Hodge-Tate decomposition of $H^{m}\left(X_{\bar{K}}, \mathbb{Q}_{p}\right) \otimes \mathbb{C}_{p}$.

1.6. Let $H_{\text {cris }}^{*}\left(X / K_{0}\right)$ denote the crystalline cohomology of the closed fiber of $X$, so we have the Berthelot-Ogus isomorphism

$$
H_{\text {cris }}^{*}\left(X / K_{0}\right) \otimes_{K_{0}} K \simeq H_{\mathrm{dR}}^{*}(X / K) .
$$

The main difficulty in proving 1.3 is to construct maps

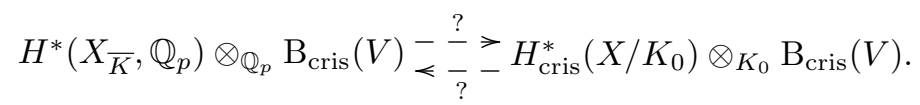

In fact it suffices to construct a map in one direction which respects the various structures and in addition is compatible with cycle classes. For then it is essentially 
formal that it is an isomorphism (as we explain in section 14. Unfortunately there is no known construction of a map relating the two cohomology theories directly, and all known proofs of 1.3 use an intermediate theory to relate the two.

There are three main approaches to proving 1.3 Let $\bar{V} \subset \bar{K}$ be the integral closure of $V$, and let $X_{\bar{V}}$ be the base change of $X$.

(i) (Syntomic cohomology). This approach has been developed by Fontaine, Messing, Hyodo, Kato, and Tsuji among others (see [18, 26, 30, 32, 49, 51]).

Here one defines certain syntomic cohomology groups $H_{\mathrm{syn}}^{*}\left(X \bar{V}, \mathscr{S}(r)_{\mathbb{Q}_{p}}\right)(r \geq$ $0)$. There are maps

$$
\alpha_{r}: H_{\mathrm{syn}}^{*}\left(X_{\bar{V}}, \mathscr{S}(r)_{\mathbb{Q}_{p}}\right) \rightarrow H^{*}\left(X_{\bar{K}}, \mathbb{Q}_{p}(r)\right)
$$

and

$$
\beta_{r}: H_{\mathrm{syn}}^{*}\left(X_{\bar{V}}, \mathscr{S}(r)_{\mathbb{Q}_{p}}\right) \rightarrow H_{\text {cris }}^{*}\left(X / K_{0}\right) \otimes_{K_{0}} \mathrm{~B}_{\text {cris }}(V) .
$$

The key result in this approach is to show that for $r>0$ the map $\alpha_{r}$ is an isomorphism. By inverting the map $\alpha_{r}$ and applying suitable Tate twists one obtains the desired morphism 1.6.1.

The proof that for $r>>0$ the map $\alpha_{r}$ is an isomorphism requires a detailed analysis of $p$-adic vanishing cycles. Let $\bar{Y}$ denote the base change of $X_{\bar{V}}$ to the residue field $\bar{k}$ of $\bar{V}$ so we have a commutative diagram

$$
X_{\bar{K}} \stackrel{\bar{j}}{\longrightarrow} X_{\bar{V}} \stackrel{\bar{i}}{\longleftarrow} \bar{Y} .
$$

By the proper base change theorem we have

$$
H^{*}\left(X_{\bar{K}}, \mathbb{Z} /\left(p^{n}\right)(r)\right) \simeq H^{*}\left(\bar{Y}, \bar{i}^{*} R \bar{j}_{*} \mathbb{Z} /\left(p^{n}\right)(r)\right) .
$$

The syntomic theory provides a crystalline interpretation of the $p$-adic vanishing cycles $\bar{i}^{*} R \bar{j}_{*} \mathbb{Z} /\left(p^{n}\right)(r)$.

Suppose $T$ is a flat and local complete intersection scheme over $W$ (such a scheme $T$ is called syntomic over $W$ ), and assume that $T$ is quasi-projective. Then we can find an immersion $i: T \hookrightarrow Z$, where $Z$ is a smooth $W$-scheme such that there exists a morphism $F_{Z}: Z \rightarrow Z$ lifting the Frobenius morphism on $Z \otimes_{W} k$, and such that the diagram

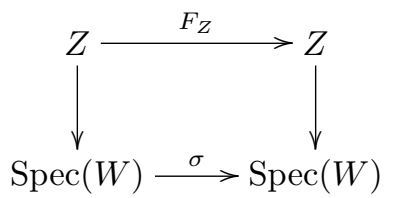

commutes (for example $Z$ can be chosen to be a suitable projective space). Let

$$
i_{n}: T_{n} \hookrightarrow Z_{n}
$$

be the morphism over $W_{n}:=W / p^{n}$ defined by reduction modulo $p^{n}$. Let $D_{n}$ be the divided power envelope of $T_{n}$ in $Z_{n}$ and let $J_{n}^{[r]}(r \geq 0)$ be the $r$-th divided power ideal of $D_{n}$ (which we view as a sheaf on $T_{1}$ ). For $r<0$ we define $J_{n}^{[r]}:=\mathcal{O}_{D_{n}}$. We then get a complex on $T_{1}$

$$
\mathbb{J}_{n}^{[r]}: J_{n}^{[r]} \rightarrow J_{n}^{[r-1]} \otimes \Omega_{Z_{n}}^{1} \rightarrow J_{n}^{[r-2]} \otimes \Omega_{Z_{n}}^{2} \rightarrow \cdots .
$$


In case $r=0$ this is simply the complex computing the crystalline cohomology of $T_{1} / W_{n}$, and in general a crystalline interpretation is given in [4, 7.2]. The map $F_{Z}$ induces a map

$$
f: \mathbb{J}_{n}^{[r]} \rightarrow \mathbb{J}_{n}^{[0]} .
$$

Define $\widetilde{\mathscr{S}_{n}}(r)_{T}$ to be the mapping fiber of the map of complexes

$$
\mathbb{J}_{n}^{[r]} \stackrel{p^{r}-f}{\longrightarrow} \mathbb{J}_{n}^{[0]} .
$$

This complex $\widetilde{\mathscr{S}}_{n}(r)_{T}$ is in fact independent of the choice of $i: T \hookrightarrow Z$ and can be defined globally using hypercovers.

Returning to our smooth proper scheme $X / V$, note that for every finite extension $V \rightarrow V^{\prime}$ the base change $X_{V^{\prime}}$ is syntomic over $W$ and therefore one obtains a complex $\widetilde{\mathscr{S}}_{n}(r)_{X_{V^{\prime}}}$ on the closed fiber of $X_{V^{\prime}}$, and hence also by pullback a complex on $\bar{Y}$. By passing to a suitable limit one then obtains a complex $\widetilde{\mathscr{S}}_{n}(r)_{X_{\bar{V}}}$ on $\bar{Y}$.

Let $\mathbb{Z} / p^{n} \mathbb{Z}(r)^{\prime}$ denote $\left(\frac{1}{p^{a} a !} \mathbb{Z}_{p}(r)\right) \otimes \mathbb{Z} / p^{n} Z$, where $r=(p-1) a+b(a \in \mathbb{Z}$ and $0 \leq b \leq p-2)$. The key result of Tsuji is then the following:

Theorem $1.7(49, \S 3])$. There is a canonical $G_{K}$-equivariant morphism

$$
\widetilde{\mathscr{S}}_{n}(r)_{X_{\bar{V}}} \rightarrow \bar{i}^{*} R \bar{j}_{*} \mathbb{Z} / p^{n} \mathbb{Z}(r)^{\prime} .
$$

If $0 \leq q \leq r$ then there exists an integer $N$ depending only on $p, q$, and $r$ such that the kernel and cokernel of the map

$$
\mathscr{H}^{q}\left(\widetilde{\mathscr{S}}_{n}(r)_{X_{\bar{V}}}\right) \rightarrow \bar{i}^{*} R^{q} \overline{j_{*}} \mathbb{Z} / p^{n} \mathbb{Z}(r)^{\prime}
$$

is annihilated by $p^{N}$.

We define

$$
H_{\text {syn }}^{*}\left(X_{\bar{V}}, \mathscr{S}(r)_{\mathbb{Q}_{p}}\right):=\mathbb{Q}_{p} \otimes \varliminf_{\longleftarrow} H^{*}\left(\bar{Y}, \widetilde{\mathscr{S}}_{n}(r)_{X_{\bar{V}}}\right) .
$$

The map $\alpha_{r}$ in 1.6 .2 is obtained from the map 1.7.1 by passing to the limit in $n$ and tensoring with $\mathbb{Q}_{p}$. It follows from the second statement in 1.7 that for $r>>0$ the map $\alpha_{r}$ is an isomorphism.

On the other hand, by construction there is a natural map

$$
H_{\text {syn }}^{*}\left(X_{\bar{V}}, \widetilde{\mathscr{S}}_{n}(r)_{X_{\bar{V}}}\right) \rightarrow H^{*}\left(\bar{Y}, \mathbb{J}_{n}^{[r]}\right),
$$

where on the right we write $\mathbb{J}_{n}^{[r]}$ for the limit of the complexes obtained from the $X_{V^{\prime}}\left(V \subset V^{\prime}\right.$ a finite extension).

It is fairly easy (at least in comparison to the proof of 1.7 ) to show that for $r \geq m$ there is a canonical isomorphism

$$
\mathrm{B}_{\text {cris }}(V)^{+} \otimes_{W} H_{\text {cris }}^{m}(X / W) \simeq \mathbb{Q}_{p} \otimes{\underset{n}{\lim }}_{n}^{*}\left(\bar{Y}, \mathbb{J}_{n}^{[0]}\right),
$$

where $\mathrm{B}_{\text {cris }}(V)^{+}$is defined in 11.1.6 (the ring $\mathrm{B}_{\text {cris }}(V)$ is a localization of $\mathrm{B}_{\text {cris }}(V)^{+}$). The map $\beta_{r}$ in 1.6 .3 is defined to be the map induced by the natural inclusion $\mathbb{J}_{n}^{[r]} \subset \mathbb{J}_{n}^{[0]}$.

(ii) (K-theory). This approach developed by Niziol [36, 37] offers perhaps the most direct relation among the two cohomology groups. The key point is that there are regulator maps

$$
c_{i j}^{\mathrm{et}}: \operatorname{gr}_{\gamma}^{i} K_{j}\left(X_{\bar{K}}, \mathbb{Z} / p^{n}\right) \rightarrow H^{2 i-j}\left(X_{\bar{K}}, \mathbb{Z} / p^{n}(i)\right),
$$


where $K_{j}\left(X_{\bar{K}}, \mathbb{Z} / p^{n}\right)$ denotes algebraic $K$-theory with coefficients, which is filtered by the $\gamma$-filtration. Using Thomason's comparison theorem between algebraic and étale $K$-theory [48, Niziol then describes the kernel and cokernel of the regulator $\operatorname{map} c_{i j}^{\mathrm{et}}$.

There is also a syntomic regulator map

$$
c_{i j}^{\mathrm{syn}}: \operatorname{gr}_{\gamma}^{i} K_{j}\left(X_{\bar{K}}, \mathbb{Z} / p^{n}\right) \rightarrow H^{2 i-j}\left(\bar{Y}, \widetilde{\mathscr{S}}_{n}(i)\right) .
$$

Upon passing to the limit in $n$, applying a suitable Tate twist, and using the map 1.6 .3 we obtain a diagram

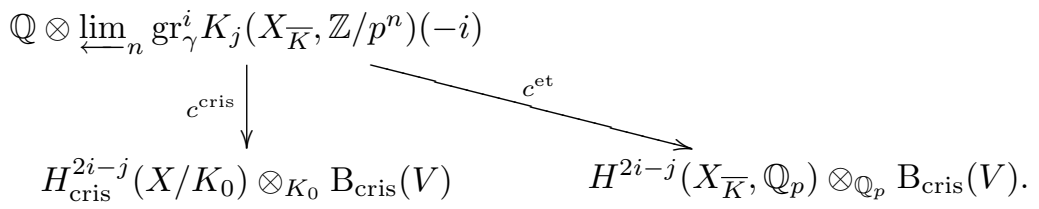

By analyzing the kernel and cokernel of $c^{\text {et }}$ Niziol then shows that this induces the desired homomorphism 1.6.1.

(iii) (Almost mathematics). This approach, which is the subject of the rest of the paper, was developed by Faltings in a series of papers $[13,14,15$. To indicate the flavor of Faltings' approach, let us give a brief sketch of his argument in the simplest case of constant coefficients and a proper scheme.

Faltings' approach is in some sense also based on a calculation of a kind of $p$-adic vanishing cycles. The key result is the almost purity theorem (see 2.17) which enables one to compute Galois cohomology in the local situation. Let $R$ be a smooth $V$-algebra and assume given an étale morphism

$$
\operatorname{Spec}(R) \rightarrow \operatorname{Spec}\left(V\left[T_{1}^{ \pm}, \ldots, T_{d}^{ \pm}\right]\right)
$$

for some integer $d$. Assume that $\operatorname{Spec}(R / p R) \neq \emptyset$ and that $R \otimes_{V} \bar{V}$ is an integral domain. Let $R_{\infty}$ be the algebra obtained by adjoining all the $p^{s}$-roots of $T_{i}$ in $R \otimes_{V} \bar{V}$ for all $i$ and $s$. Let $\bar{R} \subset \bar{K}$ denote the normalization of $R$ in the maximal field extension $\operatorname{Frac}(R) \subset L$ which is unramified over $R \otimes_{V} K$. We then have an inclusion $R_{\infty} \subset \bar{R}$. Let $\Delta$ (resp. $\Delta_{\infty}$ ) denote the Galois group of $\bar{R}$ (resp. $R_{\infty}$ ) over $R_{\bar{V}}$. The main implication (see 3.10 of Faltings' almost purity theorem is that the natural map of Galois cohomology groups

$$
H^{*}\left(\Delta_{\infty}, R_{\infty} / p R_{\infty}\right) \rightarrow H^{*}(\Delta, \bar{R} / p \bar{R})
$$

is an almost isomorphism, which means that the kernel and cokernel is annihilated by any element in the maximal ideal of $\bar{V}$. On the other hand, the Galois group $\Delta_{\infty}$ is easy to describe and in fact is isomorphic to $\mathbb{Z}_{p}(1)^{d}$. This implies that the cohomology groups $H^{*}\left(\Delta_{\infty}, R_{\infty} / p R_{\infty}\right)$ are computed by a certain explicit Koszul complex. In particular, there is a natural map

$$
R_{\bar{V}} \otimes_{\bar{V}} \dot{\Lambda}(\bar{V} / p \bar{V})^{d}(-1) \rightarrow H^{*}\left(\Delta_{\infty}, R_{\infty} / p R_{\infty}\right)
$$

which in fact is a direct summand.

This (almost) description of $H^{*}(\Delta, \bar{R} / p \bar{R})$ of course depends on the choice of chart 1.7.2. It can be made canonical by using the basis $d T_{i} / T_{i}$ for $\Omega_{R / V}^{1}$ to identify the map 1.7.3 with an almost morphism

$$
\dot{\bigwedge}\left(\Omega_{R / V}^{1} \otimes_{R} R_{\bar{V}} / p R_{\bar{V}}(-1)\right) \rightarrow H^{*}(\Delta, \bar{R} / p \bar{R})
$$


which is independent of choices.

These local calculations enable one to define a new cohomology theory which plays a similar role to the syntomic cohomology. First Faltings defines a ringed topos $\left(\mathscr{X}_{\bar{K}}, \mathcal{O}_{\mathscr{X}_{\bar{K}}}\right)$ which maps to $X_{\text {et }}$ and such that locally on $X$ when one has a map 1.7 .2 the cohomology of the structure sheaf modulo $p$ in $\mathscr{X}_{\bar{K}}$ is given by the group cohomology described above. Using these local descriptions one sees that the cohomology groups $H^{*}\left(\mathscr{X}_{\bar{K}}, \mathscr{O}_{\mathscr{X}_{\bar{K}}} /(p)\right)$ are almost finitely generated over $\bar{V} / p \bar{V}$, satisfy Poincaré duality, and there is a theory of Chern classes. There is also a natural map

$$
H^{*}\left(X_{\bar{K}}, \mathbb{Z} /(p)\right) \otimes \bar{V} \rightarrow H^{*}\left(\mathscr{X}_{\bar{K}}, \mathscr{O}_{\mathscr{X}_{\bar{K}}} /(p)\right)
$$

which one then shows is an almost isomorphism.

To go from this isomorphism to a comparison isomorphism between étale and de Rham cohomology then requires a number of steps. Principal among them are the following:

(1) Recall (see section 11) that the ring $\mathrm{B}_{\text {cris }}(V)$ is obtained by localization from a $W$-algebra $\mathrm{A}_{\text {cris }}(V)$ with $G_{K}$-action and Frobenius automorphism. The ring $\mathrm{A}_{\text {cris }}(V)$ contains certain ideals $\mathfrak{m}_{\alpha}$ (where $\alpha$ runs over positive elements of $\mathbb{Z}[1 / p]$ ), and one can consider almost mathematics over the ring $\mathrm{A}_{\text {cris }}(V)$ (and hence also over $\mathrm{B}_{\text {cris }}(V)$ ). A map of $\mathrm{A}_{\text {cris }}(V)$-modules $M \rightarrow N$ is an almost isomorphism if the kernel and cokernel are annihilated by every $\mathfrak{m}_{\alpha}$ for all $\alpha$.

To pass from a mod $p$ theory to a $p$-adic theory, one first replaces the sheaf $\mathscr{O}_{\mathscr{X}_{\bar{K}}} /(p)$ in the above with a suitable sheaf $\mathscr{A}_{\text {cris }}$ defined using the same construction as the construction of $\mathrm{A}_{\text {cris }}(V)$, and then shows that there is an almost isomorphism of $\mathrm{A}_{\text {cris }}(V)$-modules

$$
H^{*}\left(X_{\bar{K}}, \mathbb{Q}_{p}\right) \otimes_{\mathbb{Q}_{p}}\left(\mathrm{~A}_{\text {cris }}(V)_{\mathbb{Q}_{p}}\right) \rightarrow H^{*}\left(\mathscr{X}_{\bar{K}}, \mathscr{A}_{\text {cris }}\right) \otimes \mathbb{Q} .
$$

In fact this is not quite correct, and one has to work instead with the formal completion $\widehat{X}$ of $X$ along the closed fiber and a variant topos $\widehat{\mathscr{X}_{\bar{K}}}$ instead of $\mathscr{X}_{\bar{K}}$.

(2) Construct a map

$$
H_{\text {cris }}^{*}\left(X / K_{0}\right) \otimes_{K_{0}} \mathrm{~B}_{\text {cris }}(V) \rightarrow H^{*}\left(\mathscr{X}_{\bar{K}}, \mathscr{A}_{\text {cris }}\right) \otimes_{\mathrm{A}_{\text {cris }}(V)} \mathrm{B}_{\text {cris }}(V)
$$

thereby obtaining an almost morphism of $\mathrm{B}_{\text {cris }}(V)$-modules

$$
H_{\text {cris }}^{*}\left(X / K_{0}\right) \otimes_{K_{0}} \mathrm{~B}_{\text {cris }}(V) \rightarrow H^{*}\left(X_{\bar{K}}, \mathbb{Q}_{p}\right) \otimes_{\mathbb{Q}_{p}} \mathrm{~B}_{\text {cris }}(V)
$$

which by the usual argument using Chern classes will be an almost isomorphism.

(3) Lift this almost isomorphism to an actual isomorphism over $\mathrm{B}_{\text {cris }}(V)$.

1.8. Our aim in this paper is to give a detailed account of Faltings' method in the case of a variety with good reduction (and also an open variant) using the basics of almost mathematics and the almost Purity theorem as a "black box" (the interested reader should consult the very thorough treatment of the necessary almost mathematics in [21]). We pay particular attention to the $\mathbb{Q}_{p}$-theory which is not discussed in detail in the literature and consider non-constant coefficients (the ability to deal with nonconstant coefficients is one of the big advantages of Faltings' approach). 
1.9. The paper is organized as follows.

In section 2 we give the basic definitions of almost mathematics and state the purity theorem in the form needed in this paper. The main result is 2.17.

As mentioned above, the importance of the purity theorem is that it enables one to (almost) compute certain Galois cohomology groups. We perform these computations in sections 3 and 4 . The main results are 3.11 and 4.7. We also consider in this section cohomology with compact support using the theory of logarithmic structure. These computations are the key local results needed for Faltings' approach to $p$-adic Hodge theory.

We then begin the necessary foundational work for our study of the topos $\mathscr{X}_{\bar{K}}^{o}$ which will serve as the intermediary between étale and crystalline cohomology.

It is well-known (see [1, XI §3]) that if $Y / \bar{K}$ is a smooth $\bar{K}$-scheme, then for every point $y \in Y(\bar{K})$ there exists an open neighborhood $U$ of $y$ such that $U$ is a $K(\pi, 1)$. This means that for any locally constant sheaf of torsion abelian groups the natural map (see section 5 for a description of this map)

$$
H^{*}\left(\pi_{1}(U, y), F\right) \rightarrow H^{*}\left(U_{\text {et }}, F\right)
$$

is an isomorphism, where $H^{*}\left(\pi_{1}(U, y), F\right)$ denotes the continuous group cohomology of $F$ viewed as a representation of $\pi_{1}(U, y)$. In Faltings' approach a generalization of this result is needed. Namely, if $X / V$ is a smooth proper scheme then we need that for any geometric point $\bar{x} \rightarrow X$ of the closed fiber, there exists an étale neighborhood $U$ of $\bar{x}$ such that the geometric generic fiber $U_{\bar{K}}$ of $U$ is a $K(\pi, 1)$. We prove this result in section 5 as well as variants for open varieties.

We then introduce the topos $\mathscr{X}_{\bar{K}}$ and its open variant $\mathscr{X}_{\bar{K}}^{o}$ in section 6 . In the proper case there are morphisms of topoi

$$
\nu_{X}: \mathscr{X}_{\bar{K}} \rightarrow X_{\text {et }}
$$

and

$$
u_{X}: X_{\bar{K}, \text { et }} \rightarrow \mathscr{X}_{\bar{K}}
$$

Any locally constant sheaf $L$ of $\mathbb{Z} /\left(p^{r}\right)$-modules on $X_{\bar{K}}$ defines a sheaf $\mathscr{L}$ on $\mathscr{X}_{\bar{K}}$ such that $\nu_{X}^{*} \mathscr{L}=L$. Furthermore we show in section 6 that the natural map

$$
H^{*}\left(\mathscr{X}_{\bar{K}}, \mathscr{L}\right) \rightarrow H^{*}\left(X_{\bar{K}}, L\right)
$$

is an isomorphism. On the other hand, the topos $\mathscr{X}_{\bar{K}}$ has a structure sheaf $\mathscr{O}_{\mathscr{X}_{\bar{K}}}$ and there is a natural map

$$
H^{*}\left(\mathscr{X}_{\bar{K}}, \mathscr{L}\right) \rightarrow H^{*}\left(\mathscr{X}_{\bar{K}}, \mathscr{L} \otimes \mathscr{O}_{\mathscr{X}_{\bar{K}}}\right) .
$$

Perhaps the main result in the whole theory is 6.16 which implies that this map is an almost isomorphism (and in above situation provides the isomorphism 1.7.4).

The proof of 6.16 occupies the following two sections 7 and 8 . The introduction of cohomology with compact support poses some additional technical problems. In particular, we spend some time explaining how to compute compactly supported cohomology using group cohomology in section 7 . Then in section 8 we prove 6.16 by developing the necessary theory of Chern classes and verifying that the comparison maps between the various cohomology theories are compatible with Chern classes. For technical reasons we consider in this section a more general theory of cohomology with partial compact support along a boundary.

We then turn to lifting this mod $p$ result to a theory with $\mathbb{Q}_{p}$-coefficients. In section 9 we introduce a variant $\widehat{\mathscr{X}_{\bar{K}}^{o}}$ of the topos $\mathscr{X}_{\bar{K}}^{o}$ which is necessary to deal 
with $\mathbb{Q}_{p}$-coefficients. The main point is to replace $X$ by its $p$-adic completion. Fortunately the almost purity theorem also holds in this context and as we prove in 9.4 the cohomologies of $\widehat{\mathscr{X}_{K}^{o}}$ and $\mathscr{X}_{K}^{o}$ are almost isomorphic.

Then in sections 10 through 15 we explain Faltings' proof of the $C_{\text {crys }}-$ conjecture of Fontaine relating $p$-adic étale cohomology and crystalline cohomology.

Locally the comparison map is obtained by on the one hand computing crystalline cohomology using an embedding into a smooth scheme, and on the other hand computing étale cohomology using group cohomology. Of course to define the map globally requires simplicial techniques. In section 10 we explain how to compute étale cohomology using group cohomology using simplicial techniques (the only real issue is how to deal with base points for simplicial schemes).

In section 11 we review the necessary facts about Fontaine's rings $A_{c r i s}$ and $\mathrm{B}_{\text {cris }}$. We also consider a certain localization $\widetilde{B}_{\text {cris }}$ of $\mathrm{B}_{\text {cris }}$ which arises when one considers almost mathematics over $\mathrm{B}_{\text {cris }}$.

In section 12 we extend our earlier computations of cohomology in the topos $\mathscr{X}_{\bar{K}}^{o}$ to cohomology with coefficients in sheafified versions of Fontaine's rings $\mathrm{A}_{\text {cris }}$ and $\mathrm{B}_{\text {cris }}$. The main point is to show that the notion of almost isomorphism behaves well upon taking various inverse limits.

Then in 13 we define the transformation from crystalline to étale cohomology over $\widetilde{\mathrm{B}}_{\text {cris }}$ and state the comparison theorem over $\widetilde{\mathrm{B}}_{\text {cris }}$ (see 13.21), and then in section 14 we complete the proof of the comparison theorem over $\mathrm{B}_{\text {cris }}$. Again the key point is to show that the comparison map is compatible with Chern classes.

Fnally we explain in section 15 how to pass from the comparison theorem over $\widetilde{\mathrm{B}}_{\text {cris }}$ to the comparison theorem over $\mathrm{B}_{\text {cris }}$. This follows from some formal properties of these rings and Berger's theorem that any de Rham representation is potentially semistable [3, 0.7]. The main result is 15.5 .

REMARK 1.10. The results of Faltings discussed in this paper suffice for the applications we studied in 41 .

REMARK 1.11. Faltings has proven a version of the purity theorem in the substantially more general case of a variety with a certain kind of toroidal reduction [13. section 2b] (in particular it applies to varieties with semistable reduction). This enables Faltings to prove the $C_{\mathrm{st}}$-conjecture of Fontaine. While the main ideas in this more general context remain the same, there are several additional technical complications and for the sake of exposition we restrict ourselves to the good reduction case in this paper.

Also as discussed in [13, p. 258] there are some additional subtleties in developing the $\mathbb{Q}_{p}$-theory for coefficients in the semistable reduction case. The main problem lies with the construction of the so-called Hyodo-Kato isomorphism which requires certain finiteness properties of crystalline cohomology over the divided power envelope of the closed immersion $\operatorname{Spec}(V / p V) \hookrightarrow \operatorname{Spec}(W[t])$ obtained by sending $t$ to the class of a uniformizer $\pi \in V$. As this divided power envelope is not noetherian such finiteness of cohomology does not follow from standard techniques. In the case of constant coefficients the necessary finiteness properties follow from a comparison with the de Rham-Witt complex, but for general coefficients this method does not apply.

1.12. Prerequisites. In order to study open varieties, we work in this paper with logarithmic geometry in the sense of Fontaine, Illusie, and Kato [29. This is 
not necessary for the proper case, so the reader not familiar with this theory can still read the paper restricting themselves to the case of empty boundary divisor. For the discussion of the comparison theorem the reader is assumed familiar with crystalline cohomology as well as the convergent topos [39], 45].

1.13. Acknowledgements. It is a pleasure to thank A. Abbes for encouragement to write up these notes on Faltings' papers, and for pointing out several important corrections in an earlier version. I initially learned about the approach to $p$-adic Hodge theory using almost mathematics during a visit to U. Paris-Sud during summer of 2003. I would like to thank L. Illusie for the invitation and helpful conversations, and O. Gabber and T. Tsuji for very interesting lectures. The paper also benefitted from very helpful comments from the referees.

\section{Almost mathematics and the purity theorem}

2.1. Fix a ring $\bar{V}$ together with a sequence of principal ideals $\mathfrak{m}_{\alpha} \subset \bar{V}$ indexed by the positive elements $\Lambda_{+}$of some subgroup $\Lambda \subset \mathbb{Q}$ which is dense in $\mathbb{R}$ and contains 1. Let $\pi$ denote a generator of $\mathfrak{m}_{1}$, and for $\alpha \in \Lambda_{+}$let $\pi^{\alpha}$ denote a generator of $\mathfrak{m}_{\alpha}$. We assume that $\pi^{\alpha}$ is not a zero-divisor in $\bar{V}$ and that for every $\alpha, \beta \in \Lambda_{+}$there exists a unit $u \in \bar{V}^{*}$ such that $\pi_{\alpha} \cdot \pi_{\beta}=u \pi_{\alpha+\beta}$. We let $\mathfrak{m}$ denote the ideal $\cup_{\alpha>0} \mathfrak{m}_{\alpha}$.

Observe that since each $\pi^{\alpha}$ is a nonzero divisor in $\bar{V}$ the ideal $\mathfrak{m}_{\alpha}$ is a flat $\bar{V}$-module. This implies that $\mathfrak{m}$ is also a flat $\bar{V}$-module, as it is the filtering direct limit of the flat $\bar{V}$-modules $\mathfrak{m}_{\alpha}$. This implies in particular that the multiplication map $\mathfrak{m} \otimes_{\bar{V}} \mathfrak{m} \rightarrow \mathfrak{m}$ is an isomorphism.

EXAMPLE 2.2. The most important example for this paper is the following. Let $V$ be a complete discrete valuation ring of mixed characteristic $(0, p)$, field of fractions $K$, and perfect residue field $k$. Let $\bar{K}$ be an algebraic closure of $K$ and let $\bar{V}$ be the integral closure of $V$ in $\bar{K}$. We then take $\pi$ to be a uniformizer of $V$, $\Lambda=\mathbb{Q}$, and $\mathfrak{m}_{\alpha}$ the ideal of elements of valuation $\geq \alpha$.

2.3. Let $\bar{V}$ - Mod denote the category of $\bar{V}$-modules, and let $\Sigma \subset \bar{V}-\operatorname{Mod}$ denote the full subcategory of modules annihilated by $\mathfrak{m}$. The category $\Sigma$ is a Serre subcategory in the sense of [23, Chapitre III, §1], and therefore one can form the quotient category of $\bar{V}-$ Mod by the category $\Sigma$. We denote this quotient category by $\bar{V}^{a}-$ Mod. By [23, Chapitre III, Proposition 1] the category $\bar{V}^{a}-\operatorname{Mod}$ is an abelian category. The objects of $\bar{V}^{a}-$ Mod are called almost $\bar{V}$-modules and the objects of $\Sigma$ are called almost zero. It follows immediately from the definition that the tensor structure on $\bar{V}-$ Mod induces a tensor structure on $\bar{V}^{a}-$ Mod. There is a natural localization functor

$$
\bar{V}-\operatorname{Mod} \rightarrow \bar{V}^{a}-\operatorname{Mod}
$$

which is compatible with the tensor structure.

Morphisms in $\bar{V}^{a}-$ Mod have the following simple description. If $M \in \bar{V}-\operatorname{Mod}$, then the category of almost isomorphisms $\phi: M^{\prime} \rightarrow M$ has an initial object given by the morphism $\mathfrak{m} \otimes M \rightarrow M$. From this and the definition of the localized category $\bar{V}^{a}-$ Mod given in [23. Chapitre III, §1] we have

$$
\operatorname{Hom}_{\bar{V}^{a}-\operatorname{Mod}}(M, N)=\operatorname{Hom}_{\bar{V}-\operatorname{Mod}}(\mathfrak{m} \otimes M, N)
$$

for any $M, N \in \bar{V}-$ Mod. 
Definition 2.4. Let $R$ be a $\bar{V}$-algebra and let $M$ be an $R$-module.

(i) $M$ is almost projective if $\operatorname{Ext}_{R}^{i}(M, N) \in \Sigma$ for all $R$-modules $N$ and $i>0$.

(ii) $M$ is almost flat if $\operatorname{Tor}_{i}^{R}(M, N) \in \Sigma$ for all $R$-modules $N$ and $i>0$.

(iii) $M$ is almost faithfully flat if $M$ is almost flat and for any two $R$-modules $N$ and $N^{\prime}$ the map

$$
\operatorname{Hom}_{R}\left(N, N^{\prime}\right) \rightarrow \operatorname{Hom}_{R}\left(N \otimes M, N^{\prime} \otimes M\right)
$$

has almost zero kernel.

(iv) $M$ is almost finitely generated (resp. almost finitely presented) if for every $\alpha \in \Lambda_{+}$there exists a finitely generated (resp. finitely presented) $R$-module $N_{\alpha}$ and maps $\psi_{\alpha}: N_{\alpha} \rightarrow M$ and $\phi_{\alpha}: M \rightarrow N_{\alpha}$ such that $\psi_{\alpha} \circ \phi_{\alpha}=\pi^{\alpha}$. id and $\phi_{\alpha} \circ \psi_{\alpha}=\pi^{\alpha} \cdot$ id.

Lemma 2.5. If $f: A \rightarrow B$ is a morphism of $\bar{V}$-algebras whose underlying morphism of $\bar{V}$-modules is almost faithfully flat, then for any $A$-module $M$ the natural map $M \rightarrow M \otimes_{A} B$ is almost injective.

Proof. Let $K$ be the kernel of $M \rightarrow M \otimes_{A} B$. We need to show that the inclusion $K \hookrightarrow M$ is the zero map. For this it suffices to show that $K \otimes_{A} B \rightarrow$ $M \otimes_{A} B$ is the zero map. This follows from noting that we have a diagram

$$
K \otimes_{A} B \stackrel{a}{\longrightarrow} M \otimes_{A} B \stackrel{b}{\longrightarrow} M \otimes_{A} B \otimes_{A} B \stackrel{c}{\longrightarrow} M \otimes_{A} B,
$$

where $c$ is the map obtained from multiplication $B \otimes_{A} B \rightarrow B, b \circ a$ is the zero map, and $c \circ b$ is the identity.

2.6. Let $A$ be a $\bar{V}$-algebra, let $P$ be an $A$-module, and let $\omega_{P / A}$ denote the canonical morphism

$$
P \otimes_{A} \operatorname{Hom}(P, A) \rightarrow \operatorname{Hom}(P, P)
$$

By [21, 2.4.29 (1b)], if $P$ is almost finitely generated and almost projective then the map $\omega_{P / A}$ is an almost isomorphism. In particular, if

$$
\operatorname{ev}_{P / A}: P \otimes_{A} \operatorname{Hom}(P, A) \rightarrow A
$$

is the evaluation morphism then we obtain an almost trace map in $\bar{V}^{a}-\operatorname{Mod}$

$$
\operatorname{Tr}_{P / A}: \operatorname{Hom}(P, P) \rightarrow A
$$

by sending $\varphi: P \rightarrow P$ to $\operatorname{ev}_{P / A}\left(\omega_{P / A}^{-1}(\varphi)\right)$.

Definition 2.7. A morphism $A \rightarrow B$ of $\bar{V}$-algebras is an almost étale covering if the following two conditions hold:

(i) $B$ is almost finitely generated, almost faithfully flat, and almost projective as an $A$-module,

(ii) $B$ is almost finitely generated and almost projective as a $B \otimes_{A} B$-module.

2.8. Let $A \rightarrow B$ be an almost étale covering. For every $b \in B$ multiplication by $b$ induces a morphism of $A$-modules $\mu_{b}: B \rightarrow B$. Let

$$
\operatorname{tr}_{B / A}: B \rightarrow A
$$

be the morphism in $\bar{V}^{a}-\operatorname{Mod}$ sending $b$ to $\operatorname{Tr}_{B / A}\left(\mu_{b}\right)$. 
Lemma 2.9. Let $A \rightarrow B$ be an almost étale covering. Then the trace map $\operatorname{tr}_{B / A}: B \rightarrow A$ induces an epimorphism in $\bar{V}^{a}-$ Mod. In particular, for any $a \in A$ and $\alpha>0$ the element $\pi^{\alpha} a$ is in the image of the trace map $\mathfrak{m} \otimes B \rightarrow A$ (this is the morphism in $\bar{V}$ - Mod obtained from the trace map and 2.3.2).

Proof. Let $Q$ denote the cokernel of the trace map $B \rightarrow A$. Since $A \rightarrow B$ is almost faithfully flat, the vertical arrows in the following diagram

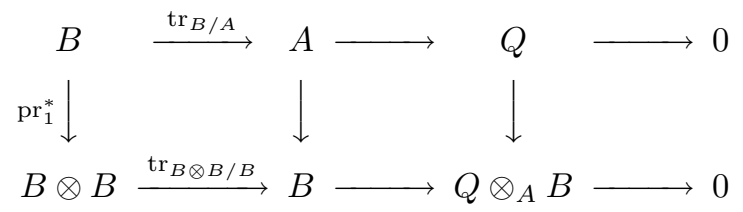

induce monomorphisms in $\bar{V}^{a}-$ Mod. It follows that to show that $Q$ is almost zero it suffices to show that the trace map $B \otimes B \rightarrow B$ is almost surjective. This is seen by observing that the composite morphism

$$
B \stackrel{\operatorname{tr}_{B / B} \otimes B}{\longrightarrow} B \otimes B \stackrel{\operatorname{tr}_{B \otimes B} \otimes B}{\longrightarrow} B
$$

is the identity in $\bar{V}^{a}-$ Mod.

Definition 2.10. Let $A \rightarrow B$ be an almost étale covering, and let $G$ be a finite group of automorphisms of $B$ over $A$. We say that $B / A$ is Galois with group $G$ if the natural map

$$
B \otimes B \rightarrow \prod_{g \in G} B, \quad b \otimes c \mapsto(\ldots, b \cdot g(c), \ldots)
$$

is an almost isomorphism.

2.11. If $A \rightarrow B$ is an almost étale Galois covering with group $G$, then $\operatorname{tr}_{B / A}$ : $B \rightarrow A$ in $\bar{V}^{a}-$ Mod can be described as follows. Momentarily let $T: B \rightarrow B$ denote the morphism $b \mapsto \sum_{g \in G} g(b)$. The map $T$ is a morphism of $A$-modules. We claim first of all that the induced morphism $T^{a}: B \rightarrow B$ in $\bar{V}^{a}-\operatorname{Mod}$ factors through $A$ (since $A \rightarrow B$ is almost faithfully flat the map $A \rightarrow B$ induces a monomorphism in $\bar{V}^{a}-$ Mod). Indeed let $Q$ denote the cokernel in the category of $A$-modules of $A \rightarrow B$ so that there is a commutative diagram with exact rows

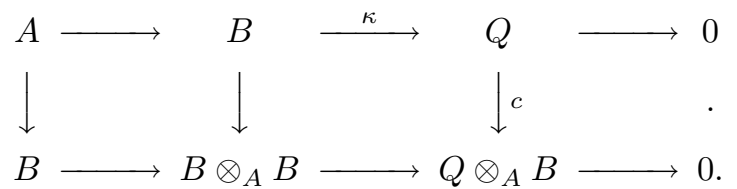

Since $A \rightarrow B$ is almost injective, it suffices to show that $c \circ \kappa \circ T$ is almost zero and hence it suffices to consider the morphism $B \rightarrow B \otimes_{A} B$. Since $B / A$ is almost Galois we can replace $B \otimes B$ by $\prod_{g \in G} B$. This reduces the problem to the case when $B=\prod_{g \in G} A$ in which case the statement is clear.

Lemma 2.12. The two morphisms $T^{a}, \operatorname{tr}_{B / A}: B \rightarrow A$ in $\bar{V}^{a}-\operatorname{Mod}$ are equal.

Proof. We think of these morphisms as actual morphisms $\mathfrak{m} \otimes B \rightarrow A$. Then using the fact that $A \rightarrow B$ is almost faithfully flat as above, one reduces to the case when $B=\prod_{g \in G} A$ in which case the result is immediate. 
2.13. In addition there is a canonical morphism $e: \bar{V} \rightarrow B \otimes B$ in $\bar{V}^{a}-\operatorname{Mod}$ defined as follows. Namely, since $B$ is almost projective viewed as a $B \otimes B$-module via the diagonal, we obtain a morphism in $\bar{V}^{a}-\operatorname{Mod}$

$$
\operatorname{End}_{B \otimes B}(B) \stackrel{\omega_{B / B \otimes B}^{-1}}{\longrightarrow} B \otimes_{B \otimes B} \operatorname{Hom}(B, B \otimes B) \stackrel{\mathrm{ev}_{B / B \otimes B}}{\longrightarrow} B \otimes B .
$$

We define $e$ to be the morphism obtained by composing this map with the natural map $\bar{V} \rightarrow \operatorname{End}_{B \otimes B}(B)$. In what follows, we will usually view $e$ as an element of $\operatorname{Hom}(\mathfrak{m}, B \otimes B)$ using 2.3.2. We often write $\pi^{\alpha} e$ for the value of $e: \mathfrak{m} \rightarrow B \otimes B$ on $\pi^{\alpha}$. Note that the composite of $e$ with the multiplication map $B \otimes B \rightarrow B$ is simply the structural morphism $\bar{V} \rightarrow B$.

Example 2.14. Suppose $A \rightarrow B$ is actually an étale morphism (in the usual sense). Then the diagonal $\operatorname{Spec}(B) \rightarrow \operatorname{Spec}(B \otimes B)$ is an open and closed immersion, and hence defined by an idempotent $e \in B \otimes B$. An elementary verification shows that the above defined morphism $\bar{V} \rightarrow B \otimes B$ is equal to the map sending 1 to $e$.

ExAmple 2.15. Let $\bar{V}$ be as in 2.2, and let $A \rightarrow B$ be a morphism of $\bar{V}$ algebras. Assume that $A$ and $B$ have no $p$-torsion. Then $(2.7$ (i)) implies that $A[1 / p] \rightarrow B[1 / p]$ is finite étale. Then for any $\alpha>0$ the element $\pi^{\alpha} e \in B \otimes B$ is equal in $B \otimes_{A} B[1 / p]$ to $\pi^{\alpha}$ times the idempotent defining the diagonal.

2.16. We can now state the version of the almost purity theorem needed for the good reduction case (see $[\mathbf{1 3}, \S 2 \mathrm{~b}]$ for the most general statement). Let $V$ be a complete discrete valuation ring of mixed characteristic $(0, p)$, let $K$ be the field of fractions of $V$, and assume that the residue field $k$ of $V$ is perfect. Let $d \geq 0$ be an integer, and consider a flat, formally étale morphism of rings

$$
V\left[T_{1}, \ldots, T_{d}\right] \rightarrow R
$$

with $R$ noetherian, and $\operatorname{Spec}(R / p R) \neq \emptyset$. Assume further that

$$
R_{\bar{V}}:=R \otimes_{V} \bar{V}
$$

is an integral domain. Let $\bar{V}\left[T_{1}^{1 / p^{\infty}}, \ldots, T_{d}^{1 / p^{\infty}}\right]$ denote the $V\left[T_{1}, \ldots, T_{d}\right]$-algebra obtained by first extending scalars to the normalization $\bar{V}$ of $V$ in $\bar{K}$, and then adjoining $p^{n}$-th roots $T_{i}^{1 / p^{n}}$ of $T_{i}$ for all $i$ and $n$. Denote by $R_{\infty}$ the base change

$$
R_{\infty}:=R \otimes_{V\left[T_{1}, \ldots, T_{d}\right]} \bar{V}\left[T_{1}^{1 / p^{\infty}}, \ldots, T_{d}^{1 / p^{\infty}}\right] .
$$

Let $R_{\infty}^{o}$ denote the localization

$$
R_{\infty}^{o}:=R_{\infty} \otimes_{V\left[T_{1}, \ldots, T_{d}\right]} V\left[T_{1}^{ \pm}, \ldots, T_{d}^{ \pm}\right]
$$

and let $R_{\infty, \bar{K}}^{o}$ denote $R_{\infty}^{o} \otimes_{V} K \simeq R_{\infty}^{o} \otimes_{\bar{V}} \bar{K}$.

Theorem 2.17 (Almost Purity). Let $R_{\infty, \bar{K}}^{o} \rightarrow S_{\bar{K}}^{o}$ denote a finite étale morphism, and let $S_{\infty}$ denote the normalization of $R_{\infty}$ in $S_{\bar{K}}^{o}$. Then the morphism $R_{\infty} \rightarrow S_{\infty}$ is an almost étale covering. If $R_{\infty, \bar{K}}^{o} \rightarrow S_{\bar{K}}^{o}$ is Galois then $R_{\infty} \rightarrow S_{\infty}$ is also an almost étale Galois covering.

REMARK 2.18. Throughout the remainder of the paper we will repeatedly return to the setup of 2.16. In what follows, if $T \rightarrow \operatorname{Spec}(R)$ is a morphism of schemes, then we write $T^{o}$ for the fiber product

$$
T^{o}:=T \times_{\operatorname{Spec}\left(V\left[T_{1}, \ldots, T_{d}\right]\right)} \operatorname{Spec}\left(V\left[T_{1}^{ \pm}, \ldots, T_{d}^{ \pm}\right]\right) .
$$

If $T$ is affine, say $T=\operatorname{Spec}(A)$, we also write $A^{o}$ for the coordinate ring of $T^{o}$. 
2.19. In order to deal systematically with cohomology, it will also be useful to have an almost version of the derived category. Let $(T, A)$ be a ringed topos with $A$ a sheaf of $\bar{V}$-algebras. As in the punctual case discussed above, define an object $M \in A-$ Mod to be almost zero if $M$ is annihilated by $\pi^{\alpha}$ for all $\alpha \in \Lambda_{+}$. Let $A^{a}-$ Mod denote the localization of $A$ - Mod by the full subcategory of almost zero modules.

Let $D(A)$ denote the derived category of $A$-modules in $T$, and let $\Sigma$ denote the multiplicative set of morphisms in $D(A)$ which induce almost isomorphisms on cohomology sheaves. By [53, 10.3.7] one can then form the localized category $\Sigma^{-1} D(A)$ which we denote by $\widetilde{D}(A)$. The category $\widetilde{D}(A)$ is naturally a triangulated category and the localization functor $D(A) \rightarrow \widetilde{D}(A)$ is a triangulated functor. Furthermore, the $t$-structure on $D(A)$ induces a $t$-structure on $\widetilde{D}(A)$ whose heart is the category $A^{a}-$ Mod. For any $* \in\{+,-, b,[a, b]\}$ we also have the corresponding subcategories $\widetilde{D}^{*}(A) \subset \widetilde{D}(A)$.

Let $f:\left(T^{\prime}, A^{\prime}\right) \rightarrow(T, A)$ be a morphism of ringed topoi with $A^{\prime}$ also a $\bar{V}$ algebra. If $f: M \rightarrow N$ is a morphism in $D\left(A^{\prime}\right)$ inducing almost isomorphisms on all cohomology sheaves, then the morphism $R f_{*}: R f_{*} M \rightarrow R f_{*} N$ also induces almost isomorphisms on all cohomology sheaves. It follows that the functor $R f_{*}$ descends to a functor, which we denote by the same symbols, $R f_{*}: \widetilde{D}\left(A^{\prime}\right) \rightarrow \widetilde{D}(A)$.

Remark 2.20. As in 2.3.2, for any objects $M, N \in A$-Mod there is a canonical isomorphism

$$
\operatorname{Hom}_{A^{a}-\operatorname{Mod}}(M, N) \simeq \operatorname{Hom}_{A-\operatorname{Mod}}(\mathfrak{m} \otimes M, N) .
$$

This implies in particular that morphisms in $A^{a}$ - Mod can be constructed locally in $T$ by gluing.

We conclude this section with the following three results about projective systems which will be used in what follows.

LEMma 2.21. Let $\rho .:\left\{F_{n}\right\} \rightarrow\left\{G_{n}\right\}$ be a morphism of projective systems of $\bar{V}$-modules such that for every $n$ the map $\rho_{n}: F_{n} \rightarrow G_{n}$ is an almost isomorphism. Then $\rho: \underset{\lim }{\longleftarrow} F_{n} \rightarrow \underset{\lim }{\longleftarrow} G_{n}$ is an almost isomorphism.

Proof. Say $f=\left\{f_{n}\right\} \in \lim F_{n}$ maps to zero under $\rho$, and let $\epsilon \in \Lambda^{+}$be an element. Then for every $n$ the element $f_{n} \in F_{n}$ is annihilated by $\pi^{\epsilon}$ whence $f$ is also annihilated by $\pi^{\epsilon}$. Therefore $\rho$ is almost injective.

For almost surjectivity, consider an element $g=\left\{g_{n}\right\} \in \lim G_{n}$. Choose any element $\epsilon \in \Lambda_{+}$and write $\epsilon=\epsilon_{0}+\epsilon_{1}$ with $\epsilon_{0}, \epsilon_{1}$ also in $\Lambda_{+}$. Choose for each $n$ an element $f_{n} \in F_{n}$ such that $\rho_{n}\left(f_{n}\right)=\pi^{\epsilon_{0}} g_{n}$. If $\bar{f}_{n+1} \in F_{n}$ denotes the image of $f_{n+1}$, then the element $\bar{f}_{n+1}-f_{n}$ is in the kernel of $\rho_{n}$ and is therefore annihilated by $\pi^{\epsilon_{1}}$. It follows that the sequence $\left\{\pi^{\epsilon_{1}} f_{n}\right\}$ defines an element of $\lim F_{n}$ mapping to $\pi^{\epsilon} g$.

Lemma 2.22. Let $\left\{C_{n}^{\bullet}\right\}$ be a projective systems of bounded below complexes of $\bar{V}$-modules such that for every $n$ and $j$ the map $C_{n+1}^{j} \rightarrow C_{n}^{j}$ is almost surjective. Then the cone of the natural map

$$
\varliminf_{\longleftarrow} C_{n}^{\bullet} \rightarrow R \varliminf_{\longleftarrow} C_{n}^{\bullet}
$$

is almost zero. 
PROOF. The spectral sequence associated to the "stupid filtration" on the complex of projective systems $\left\{C_{n}^{\bullet}\right\}$ gives a spectral sequence [10, 1.4.5]

$$
E_{1}^{p q}=R^{q} \varliminf_{\longleftarrow} C^{p} \Longrightarrow R^{p+q} \varliminf_{\longleftarrow} C^{\bullet} .
$$

From this it follows that it suffices to show that $R^{i} \lim C^{p}$ is almost zero for all $p$ and all $i>0$. This reduces the proof to the case when $\left\{C_{n}^{\bullet}\right\}$ is just a projective system of $\bar{V}$-modules with almost surjective transition maps. For every $n$, let $D_{n}$ denote the cokernel of the inclusion $\mathfrak{m} C_{n} \subset C_{n}$. Then each $D_{n}$ is almost zero so for every $i$ the map

$$
R^{i} \varliminf_{\longleftarrow}\{\mathfrak{m} C .\} \rightarrow R^{i} \varliminf_{\longleftarrow}\{C .\}
$$

is an almost isomorphism. The projective system $\mathfrak{m} C$. has surjective transition maps. Indeed if $\pi^{\alpha} c \in C_{n}$ for some $\alpha \in \Lambda_{+}$, then write $\alpha=\alpha_{1}+\alpha_{2}$ with $\alpha_{1}, \alpha_{2} \in$ $\Lambda_{+}$. By our assumptions the element $\pi^{\alpha_{1}} c$ lifts to $C_{n+1}$ so $\pi^{\alpha} c$ lifts to $\mathfrak{m} C_{n+1}$. It

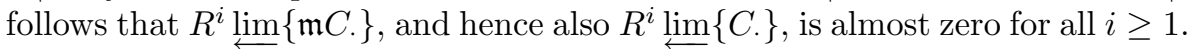

Lemma 2.23. Let $\left\{C_{n}^{\bullet}\right\}$ be a projective system of bounded below complexes of $\bar{V}$-modules such that each of the maps $C_{n+1}^{j} \rightarrow C_{n}^{j}$ is almost surjective. Assume that for every integer $i$ the module $R^{1} \lim _{\longleftarrow} H^{i}\left(C_{n}\right)$ is almost zero. Then the natural map

$$
H^{i}\left(\varliminf_{\varlimsup} C_{n}\right) \rightarrow \varliminf_{\lim ^{i}} H^{(}\left(C_{n}\right)
$$

is an almost isomorphism.

Proof. The stupid filtration on the complex of projective systems $\left\{C_{n}\right\}$ induces a spectral sequence (the "second spectral sequence of hypercohomology") [10, 1.4.5]

$$
E_{2}^{p q}=R \varliminf^{q} H^{p}\left(C_{n}\right) \Longrightarrow H^{p+q}\left(R \varliminf_{\longleftarrow} C_{n}\right) .
$$

By our assumptions $R \lim ^{q} H^{p}\left(C_{n}\right)$ is almost zero unless $q=0$ so this shows that the right side of 2.23.1 is almost isomorphic to $H^{i}\left(R \lim C_{n}\right)$. On the other hand, by the preceding lemma $\lim C_{n}$ is almost isomorphic to $R \lim C_{n}$ which implies the lemma.

\section{Galois cohomology}

The main application of the Almost Purity Theorem is to the computation of Galois cohomology.

The key lemma is the following:

Lemma 3.1. Let $G$ be a finite group, $A$ a ring, and $f: A \rightarrow B$ an $A$-algebra with $G$-action over $A$. For $b \in B$ define the trace of $b$, denoted $\operatorname{tr}(b)$, to be the element $\operatorname{tr}(b):=\sum_{g \in G} g(b)$. Let $b \in B$ be an element with $\operatorname{tr}(b)$ in the image of $A$, and let a map to $\operatorname{tr}(b)$. Then for any $B$-module $M$ with semi-linear action of $G$ and $i>0$, the cohomology groups $H^{i}(G, M)$ are annihilated by a.

Proof. First let us make some standard remarks about injective objects in the category of $B$-modules with semi-linear action of $G$.

Let $B\{G\}$ be the non-commutative ring with basis $\left\{e_{g}\right\}_{g \in G}$ and multiplication given by $\left(b e_{g}\right) \cdot\left(b^{\prime} e_{g^{\prime}}\right)=b g\left(b^{\prime}\right) e_{g g^{\prime}}$. Then the category of $B$-modules with semilinear action of $G$ is canonically equivalent to the category of left $B\{G\}$-modules. 
In particular, this category has enough injectives. In fact, injective modules can be constructed as follows. The forgetful functor

$$
F:(\text { left } B\{G\} \text {-modules }) \rightarrow \text { (left } B \text {-modules })
$$

has a right adjoint $I$ given by sending a left $B$-module $M$ to

$$
I(M):=\operatorname{Hom}_{\text {left } B \text {-modules }}(B\{G\}, M) .
$$

Here $I(M)$ is given the structure of a left $B\{G\}$ module by having $e \in B\{G\}$ act by sending $\varphi: B\{G\} \rightarrow M$ to the map

$$
\tau \mapsto \varphi(\tau \cdot e), \quad \tau \in B\{G\} .
$$

Since the functor $F$ is exact, the functor $I$ takes injectives to injectives. It follows that if $M$ is a left $B\{G\}$-module, and $F(M) \hookrightarrow N$ is an inclusion of the underlying $B$-module into an injective $B$-module, then the resulting inclusion $M \hookrightarrow I(N)$ is an inclusion of $M$ into an injective $B\{G\}$-module. Note also that $I$ is an exact functor. In particular,

Applying this discussion with $B=\mathbb{Z}$, we see that if $M$ is any abelian group then since $I$ is an exact functor taking injectives to injectives we have

$$
H^{i}(G, I(M))=0, \text { for } i>0 .
$$

Also note that for an arbitrary ring with $G$-action $B$ and left $B$-module $M$, the underlying $\mathbb{Z}\{G\}$-module of $I(M)$ is isomorphic to $\operatorname{Hom}_{\mathbb{Z}}(\mathbb{Z}\{G\}, M)$. In particular, if $N$ is an injective object in the category of left $B\{G\}$-modules then

$$
H^{i}(G, N)=0 \text {, for } i>0 \text {. }
$$

We conclude that if $M$ is a $B$-module with semi-linear action of $G$, then $H^{*}(G, M)$ can be computed as follows: Choose an injective resolution $M \rightarrow I^{*}$ in the category of left $B\{G\}$-modules, and let $I^{\vee}, G$ be the complex obtained by taking $G$-invariants. Then

$$
H^{i}(G, M)=H^{i}\left(I^{,}, G\right) .
$$

Returning to the proof of the lemma, let $M \rightarrow I^{\cdot}$ be an injective resolution by $B$-modules with semi-linear $G$-action. Let $m \in\left(I^{i}\right)^{G}$ be an element defining a class in $H^{i}(G, M)$. Then since $I^{\prime}$ is a resolution there exists an element $m^{\prime} \in I^{i-1}$ mapping to $m$. Then $\operatorname{tr}\left(b m^{\prime}\right) \in\left(I^{i-1}\right)^{G}$. On the other hand, the image of $\operatorname{tr}\left(b m^{\prime}\right)$ in $I^{i}$ is equal to $\operatorname{tr}(b m)$. Since $m$ is $G$-invariant, we have $\operatorname{tr}(b m)=\operatorname{tr}(b) \cdot m=f(a) \cdot m$. Therefore $f(a) \cdot m$ is a boundary which proves the lemma.

3.2. Let $\bar{V}$ be as in 2.2 and let $f: A \rightarrow B$ be an almost étale covering. Assume that $A$ and $B$ are integral domains flat over $\bar{V}$ and integrally closed in their fields of fractions. Then since $A \otimes_{V} K \rightarrow B \otimes_{V} K$ is étale, we get a morphism between the field of fractions $\operatorname{Frac}(A) \rightarrow \operatorname{Frac}(B)$. Assume that this field extension is Galois with group $G$. Then $G$ also acts on $B$ over $A$ since $B$ is integrally closed in its field of fractions.

Lemma 3.3. The extension $A \rightarrow B$ is Galois with group $G$.

Proof. We have to show that the map

$$
B \otimes_{A} B \rightarrow \prod_{g \in G} B, \quad b \otimes c \mapsto(\ldots, b g(c), \ldots)_{g \in G}
$$

is an almost isomorphism. Let $e_{g} \in \prod_{g \in G} B$ be the idempotent $(0, \ldots, 1,0, \ldots, 0)$ with a 1 in the $g$-th component and zeros in the other spots. We first show that 
for every $\alpha \in \Lambda_{+}$the element $\pi^{\alpha} e_{g}$ is in the image. This will imply that 3.3.1 is almost surjective.

For this note that the map 3.3.1 is naturally a $G$-equivariant morphism where $h \in G$ acts on $B \otimes_{A} B$ by $b \otimes c \mapsto b \otimes h(c)$, and on $\prod_{g \in G} B$ by sending $\left(f_{g}\right)_{g \in G}$ to the element with $g$-component $f_{g h}$. This implies that it suffices to show that for every $\alpha \in \Lambda_{+}$the element $\pi^{\alpha} e_{1}$ is in the image, where $1 \in G$ denotes the identity element. This element is in fact equal to the image of the element $\pi^{\alpha} e$ defined in 2.13 To verify this note that the map $B \rightarrow B[1 / \pi]$ is injective so it suffices to show that the image of $\pi^{\alpha} e$ in $\prod_{g \in G} B[1 / \pi]$ is equal to $\pi^{\alpha} e_{1}$ which follows from the fact that $A[1 / \pi] \rightarrow B[1 / \pi]$ is étale and Galois with group $G$. This completes the proof of the almost surjectivity of 3.3 .1 .

For the almost injectivity, let $M$ denote the kernel of 3.3.1, so we have an almost exact sequence

$$
0 \rightarrow M \rightarrow B \otimes_{A} B \rightarrow \prod_{g \in G} B \rightarrow 0 .
$$

Using this sequence and the fact that $B \otimes_{A} B$ and $\prod_{g \in G} B$ are almost flat over $A$, one sees that $M$ is almost flat over $A$.

Now observe that since $A[1 / \pi] \rightarrow B[1 / \pi]$ is étale and Galois in the usual sense, every element of $M$ is annihilated by some element $\pi^{\alpha}$ for some $\alpha \in \Lambda_{+}$. We therefore get that

$$
M=\cup_{\alpha \in \Lambda_{+}} \operatorname{Tor}_{1}^{A}\left(M, A /\left(\pi^{\alpha}\right)\right) .
$$

Now since $M$ is almost flat each of the modules $\operatorname{Tor}_{1}^{A}\left(M, A /\left(\pi^{\alpha}\right)\right)$ is almost zero, which implies that $M$ is also almost zero.

Proposition 3.4. Let $M$ be a $B$-module with semi-linear action of $G$. Then $H^{i}(G, M)$ is almost zero for all $i>0$ and $M$ is almost isomorphic to $B \otimes_{A} M^{G}$.

Proof. By 2.9 and 2.12 for any $\alpha \in \Lambda_{+}$the element $\pi^{\alpha} \in A$ is in the image of the trace map. From this and 3.1 it follows that $\pi^{\alpha}$ annihilates all higher cohomology groups.

For $\alpha \in \Lambda_{+}$, let $\pi^{\alpha} e \in B \otimes B$ be the element defined in 2.13. Write $\pi^{\alpha} e=$ $\sum_{i} b_{i} \otimes c_{i}$ with $b_{i}, c_{i} \in B$. Since $\pi^{\alpha} e$ maps to $\pi^{\alpha}$ in $B$ under multiplication we have

$$
\pi^{\alpha}=\sum_{i} b_{i} \cdot c_{i}
$$

On the other hand,

$$
\sum_{i} b_{i} \cdot g\left(c_{i}\right)=0
$$

for any nontrivial $g \in G$. Indeed this can be verified after tensoring with $K$ in which case it is immediate. Define a map

$$
r: M \rightarrow B \otimes_{A} M^{G}, \quad m \mapsto \sum_{i} b_{i} \otimes \operatorname{tr}\left(c_{i} \cdot m\right),
$$

and let $s: B \otimes_{A} M^{G} \rightarrow M$ be the natural map. Then

$$
r \circ s(b \otimes m)=\sum_{i} b_{i} \otimes \operatorname{tr}\left(c_{i} b m\right)=\sum_{i} b_{i} \otimes \operatorname{tr}\left(c_{i} b\right) m,
$$


since for $m \in M^{G}$ we have $\operatorname{tr}(b m)=\operatorname{tr}(b) m$. Now for any $\epsilon \in \Lambda_{+}$we have $\pi^{\epsilon} \operatorname{tr}\left(c_{i} b\right) \in A$ so we also have

$$
\pi^{\epsilon} \sum_{i} b_{i} \otimes \operatorname{tr}\left(c_{i} b\right) m=\pi^{\epsilon} \sum_{i} b_{i} \operatorname{tr}\left(c_{i} b\right) \otimes m .
$$

On the other hand, using 3.4 .2 we have $(3.4 .5)$

$\sum_{i} b_{i} \cdot \operatorname{tr}\left(c_{i} b\right)=\sum_{i} b_{i} \cdot\left(\sum_{g} g\left(c_{i}\right) \cdot g(b)\right)=\sum_{g} \sum_{i} b_{i} \cdot g\left(c_{i}\right) g(b)=\sum_{i} b_{i} \cdot c_{i} b=\pi^{\alpha} \cdot b$.

This shows that $\pi^{\epsilon} r \circ s$ is equal to multiplication by $\pi^{\epsilon+\alpha}$ for any $\alpha, \epsilon \in \Lambda_{+}$. The same argument shows that $r$ is $B$-linear. Computing we also find that

$$
s \circ r(m)=\sum_{i} b_{i} \cdot \operatorname{tr}\left(c_{i} \cdot m\right)=\sum_{i} \sum_{g} b_{i} \cdot g\left(c_{i}\right) g(m)=\sum_{i} b_{i} \cdot c_{i} m=\pi^{\alpha} m .
$$

To conclude this section, we record some calculations which will be used later. Let $\bar{K}$ denote an algebraically closed field of characteristic 0 , let $p$ be a prime, and for an integer $n>0$ let $\mu_{p^{n}}$ denote the group of $p^{n}$-th roots of unity in $\bar{K}$, and let $\mu_{p^{\infty}}:=\cup_{n} \mu_{p^{n}} \subset \bar{K}$. We view $\mu_{p^{\infty}}$ as a topological group with the discrete topology.

Raising to the $p$-th power defines a homomorphism $\mu_{p^{n+1}} \rightarrow \mu_{p^{n}}$ and we let $\mathbb{Z}_{p}(1)$ denote $\lim _{n} \mu_{p^{n}}$. We view $\mathbb{Z}_{p}(1)$ as a topological group with the profinite topology. Let $d \geq 1$ be an integer, and let $\Delta$ denote $\mathbb{Z}_{p}(1)^{d}$. Let $\bar{V} \subset \bar{K}$ denote a subring containing $\mu_{p^{\infty}}$. If $M$ is a $\bar{V}$-module and $n$ is an integer we write $M(n)$ for $M \otimes_{\mathbb{Z}_{p}} \mathbb{Z}_{p}(1)^{\otimes n}$ (if $n<0$ then $\mathbb{Z}_{p}(1)^{\otimes n}$ is defined to be the continuous dual of $\left.\mathbb{Z}_{p}(1)^{\otimes-n}\right)$.

Proposition 3.5. Let $\mu: \Delta \rightarrow \mu_{p^{\infty}}$ be a continuous homomorphism, $r$ a positive integer, and let $L_{\mu}$ denote the corresponding rank 1 module over $\bar{V} / p^{r} \bar{V}$ with continuous action of $\Delta$.

(i) If $\mu$ is trivial, then the $\bar{V} / p^{r} \bar{V}$-module $H^{*}\left(\Delta, L_{\mu}\right)$ is isomorphic to the exterior algebra $\wedge^{\bullet}\left(\bar{V} / p^{r} \bar{V}\right)^{d}(-1)$.

(ii) If $\mu$ is non-trivial, then there exists an integer $n>0$ such that if $\zeta$ is a primitive $p^{n}$-th root of 1 then $H^{*}\left(\Delta, L_{\mu}\right)$ is annihilated by $\zeta-1$.

Proof. For $1 \leq i \leq d$ let $\mu_{i}: \mathbb{Z}_{p}(1) \rightarrow \mu_{p^{\infty}}$ denote the restriction of $\mu$ to the $i$-th factor of $\mathbb{Z}_{p}(1)^{d}$. Then $L_{\mu}$ is isomorphic to tensor product of the representations $L_{\mu_{i}}$ of $\mathbb{Z}_{p}(1)$. By the Künneth formula A.7 it therefore suffices to prove the proposition in the case when $d=1$.

Since $\mu$ is continuous there exists an integer $n$ such that the image of $\mathbb{Z}_{p}(1)$ is contained in $\mu_{p^{n}}$. Let $\mathfrak{g}_{s}$ denote the quotient $\mathbb{Z}_{p}(1) / p^{n s} \mathbb{Z}_{p}(1)$. For each $s$ the action of $\mathbb{Z}_{p}(1)$ on $L_{\mu}$ factors through $\mathfrak{g}_{s}$ so

$$
H^{i}\left(\mathbb{Z}_{p}(1), L_{\mu}\right)=\underset{s}{\lim } H^{i}\left(\mathfrak{g}_{s}, L_{\mu}\right) .
$$

Choose a generator $\sigma \in \mathbb{Z}_{p}(1)$, and let $D: L_{\mu} \rightarrow L_{\mu}$ be the map sending $l \in L_{\mu}$ to $\sigma(l)-l$. Let $N_{s}: L_{\mu} \rightarrow L_{\mu}$ denote the map $l \mapsto \sum_{g \in \mathfrak{g}_{s}} g(l)$. Then as in 43, VIII, 
$\S 4]$ for $i>0$ the cohomology groups $H^{i}\left(\mathfrak{g}_{s}, L_{\mu}\right)$ are equal to the $i$-th cohomology group of the complex

$$
L_{\mu} \stackrel{D}{\longrightarrow} L_{\mu} \stackrel{N_{s}}{\longrightarrow} L_{\mu} \stackrel{D}{\longrightarrow} L_{\mu} \stackrel{N_{s}}{\longrightarrow} \cdots .
$$

Furthermore, the maps $H^{i}\left(\mathfrak{g}_{s}, L_{\mu}\right) \rightarrow H^{i}\left(\mathfrak{g}_{s+1}, L_{\mu}\right)$ occuring in the direct limit 3.5 .1 are given by the morphisms of complexes

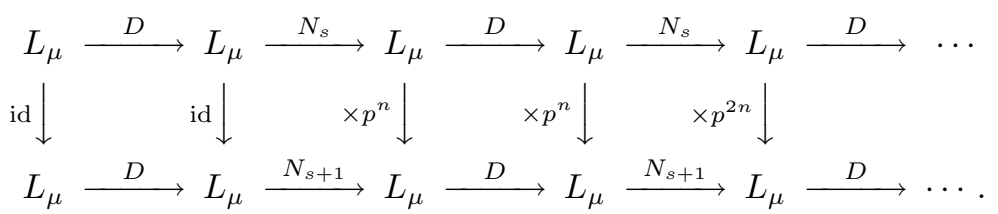

Since $L_{\mu}$ is annihilated by a power of $p$ this implies that $H^{i}\left(\mathbb{Z}_{p}(1), L_{\mu}\right)=0$ for all $i \geq 2$.

If $L_{\mu}$ is the trivial representation, then $N_{s}$ is multiplication by $p^{n s}$ and $D$ is the zero map. Since $L_{\mu}$ is annihilated by a power of $p$ it follows that $H^{1}\left(\mathbb{Z}_{p}(1), L_{\mu}\right)=$ $\bar{V} / p^{r} \bar{V}$. Now this isomorphism depends on the choice of generator $\sigma \in \mathbb{Z}_{p}(1)$. We can make this more canonical as follows. An element $\tau \in \mathbb{Z}_{p}(-1)$ can be thought of as a morphism $\tau: \mathbb{Z}_{p}(1) \rightarrow \mathbb{Z}_{p}$. For any such morphism $\tau$, we obtain an extension of $\mathbb{Z}_{p}(1)$-modules

$$
0 \rightarrow \mathbb{Z}_{p} \rightarrow E \rightarrow \mathbb{Z}_{p} \rightarrow 0
$$

where $E$ is the $\mathbb{Z}_{p}$-space $\mathbb{Z}_{p}^{2}$ with action of $\sigma \in \mathbb{Z}_{p}(1)$ given by $(a, b) \mapsto(a, b+a \cdot \tau(\sigma))$. This defines an isomorphism $\mathbb{Z}_{p}(-1) \rightarrow H^{1}\left(\mathbb{Z}_{p}(1), L_{\mu}\right)$ proving (i).

For (ii), let $\sigma \in \mathfrak{g}_{s}$ be a generator, let $a \in L_{\mu}$ be a nonzero element, and let $\zeta$ be a primitive $p^{n}$-th root of 1 . By choosing $n$ appropriately we have $\sigma(a)=\zeta^{\alpha} a$ for some positive integer $\alpha$ prime to $p$ (since $L_{\mu}$ is nontrivial). If $u \in \bar{V}^{*}$ denotes the unit $1+\zeta+\cdots+\zeta^{\alpha-1}$ then we can also write this as $D(a)=(\zeta-1) u a$. It follows that $(\zeta-1) a$ is equal to $D\left(u^{-1} a\right)$. In particular for any element $a \in L_{\mu}$ in the kernel of $N_{s}$ the corresponding cohomology class is killed by $(\zeta-1)$.

3.6. In fact the proof of 3.5 shows more. Let $\Gamma_{\Delta}$ denote the functor taking $\Delta$-invariants. Fix a basis $\sigma_{1}, \ldots, \sigma_{d}$ for $\Delta$, and let $L_{\mu_{i}}$ denote the representation of $\mathbb{Z}_{p}(1)$ obtained from $\mu$ by restricting to the $i$-th factor. Let $D_{i}: L_{\mu_{i}} \rightarrow L_{\mu_{i}}$ be the map $\sigma_{i}-1$ Then the proof of 3.5 shows that

$$
R \Gamma_{\Delta}\left(L_{\mu}\right)
$$

is represented by the tensor product of the complexes (concentrated in degrees 0 and 1)

$$
L_{\mu_{i}} \stackrel{D_{i}}{\longrightarrow} L_{\mu_{i}} .
$$

This tensor product is an example of a Koszul complex. Let $F$ be a ring, let $n \geq 0$ be an integer, and let $F\left[x_{1}, \ldots, x_{n}\right]$ be the polynomial ring over $F$. For an $F$-module $M$, we define the Koszul complex (see for example [12, §17.2])

$$
\operatorname{Kos}(M):=\operatorname{Hom}_{F\left[x_{1}, \ldots, x_{n}\right]}(\mathscr{K} \cdot M),
$$

where $\mathscr{K}^{\cdot}$ is the standard Koszul resolution of $F$ viewed as an $F\left[x_{1}, \ldots, x_{n}\right]$-algebra by sending all the $x_{i}$ to 0 . By definition, $\mathscr{K}$ is a complex concentrated in degrees 
$[-n, 0], \mathscr{K}^{-i}=\bigwedge^{i}\left(F^{n}\right)$, and the differential

$$
d: \mathscr{K}^{-i} \rightarrow \mathscr{K}^{-i+1}
$$

sends $m_{1} \wedge \cdots \wedge m_{i}$ to

$$
\sum_{j=1}^{i}(-1)^{j-1} \varphi\left(m_{j}\right) \cdot m_{1} \wedge \cdots \wedge \hat{m}_{j} \wedge \cdots \wedge m_{i},
$$

where $\varphi: F^{n} \rightarrow F$ is the map

$$
\left(a_{1}, \ldots, a_{n}\right) \mapsto \sum_{j} a_{j} x_{j} .
$$

As explained in [12, 17.15], the complex $\mathscr{K}^{\cdot}$ is self-dual in the sense that there is a canonical isomorphism

$$
\mathscr{K} \cdot[-d] \simeq \operatorname{Hom}_{F}(\mathscr{K}, F) .
$$

If $M$ is any $F\left[x_{1}, \ldots, x_{n}\right]$-module, we then get isomorphisms

$$
\begin{aligned}
\operatorname{Hom}^{\prime}(\operatorname{Kos}(M), F) & =\operatorname{Hom}\left(\operatorname{Hom}^{\prime}(\mathscr{K} \cdot M), F\right) \\
& \simeq \operatorname{Hom}(\operatorname{Hom}(\mathscr{K} \cdot F) \otimes M, F) \\
& \simeq \operatorname{Hom}\left(\mathscr{K}^{\cdot}[-d] \otimes M, F\right) \\
& \simeq \operatorname{Hom}\left(\mathscr{K}^{\cdot}[-d], \operatorname{Hom}(M, F)\right) \\
& \simeq \operatorname{Kos}(\operatorname{Hom}(M, F))[d] .
\end{aligned}
$$

Now if $L$ is any continuous representation of $\Delta$ on a finitely generated free $\bar{V} / p^{r}$-module, then a generalization of the above argument, which we leave to the reader, shows that the cohomology

$$
R \Gamma_{\Delta}(L)
$$

is represented by the $\operatorname{Koszul}$ complex $\operatorname{Kos}(L)$ (taking $F=\bar{V} / p^{r} V$ ), where we view $L$ as a module over $\bar{V} / p^{r}\left[x_{1}, \ldots, x_{d}\right]$ by choosing a basis $\sigma_{1}, \ldots, \sigma_{d}$ for $\Delta$ and letting $x_{i}$ act on $L$ by $\sigma_{i}-1$.

The duality 3.6 .2 then yields an isomorphism

$$
R \operatorname{Hom}\left(R \Gamma_{\Delta}(L), \bar{V} / p^{r}\right) \simeq R \Gamma_{\Delta}\left(\operatorname{Hom}\left(L, \bar{V} / p^{r}\right)\right)[d] .
$$

This isomorphism of course depends on the choice of basis for $\Delta$. It can be made canonical as follows.

From $3.5(\mathrm{i}))$, we get a trace map

$$
t: R \Gamma_{\Delta}\left(\bar{V} / p^{r}\right) \rightarrow \bar{V} / p^{r} \bar{V}(-d)[-d],
$$

which induces a pairing

$$
R \Gamma_{\Delta}(L) \otimes R \Gamma_{\Delta}\left(\operatorname{Hom}\left(L, \bar{V} / p^{r}\right)\right) \longrightarrow R \Gamma_{\Delta}\left(\bar{V} / p^{r}\right) \stackrel{t}{\longrightarrow} \bar{V} / p^{r}(-d)[-d],
$$

where the first map is induced by the natural pairing

$$
L \otimes \operatorname{Hom}\left(L, \bar{V} / p^{r}\right) \rightarrow \bar{V} / p^{r} .
$$

The map 3.6 .4 induces a morphism

$$
R \Gamma_{\Delta}\left(\operatorname{Hom}\left(L, \bar{V} / p^{r}\right)\right) \rightarrow R \operatorname{Hom}\left(R \Gamma_{\Delta}(L), \bar{V} / p^{r}(-d)[-d]\right),
$$

which upon choosing a basis becomes identified with the map 3.6.3. Therefore 3.6.5 is an isomorphism. 
3.7. Now let $\bar{V}, R$, etc. be as in 2.16. Recall that $\operatorname{Spec}\left(R_{K}\right)$ is assumed geometrically connected. Let $k(R)$ denote the field of fractions of $R$, fix an algebraic closure $k(R) \subset \overline{k(R)}$, let $k(R)^{u} \subset \overline{k(R)}$ denote the union of the finitely generated subfields $k(R) \subset L \subset \overline{k(R)}$ for which the normalization of $R_{K}^{o}$ in $L$ is étale over $R_{K}^{o}$. Let $\bar{R}$ denote the normalization of $R$ in $k(R)^{u}$.

Let $\eta \rightarrow \operatorname{Spec}\left(R_{K}\right)$ denote the geometric generic point $\operatorname{Spec}(\overline{k(R)})$ and let $\Delta$ denote the fundamental group $\pi_{1}\left(\operatorname{Spec}\left(R_{\bar{K}}^{o}\right), \eta\right)$. Also let $\Delta_{\infty}$ denote the Galois group of $R_{\infty}$ over $R_{\bar{V}}$. Note that $\Delta_{\infty}$ is isomorphic to $\mathbb{Z}_{p}(1)^{d}$. The natural map $R_{\infty} \rightarrow \bar{R}$ defines a homomorphism $\delta: \Delta \rightarrow \Delta_{\infty}$. Let $\Sigma$ denote its kernel.

Remark 3.8. The $R_{\infty}$-algebra $\bar{R}$ is almost faithfully flat, being a filtering direct limit of almost étale coverings of $R_{\infty}$ by 2.17

Lemma 3.9. The morphism $\delta: \Delta \rightarrow \Delta_{\infty}$ is surjective.

Proof. Let $z \in \operatorname{Spec}\left(R_{\bar{V}}\right)$ be a point in the closed fiber, let $R^{\prime}$ denote the Zariski local ring of $z$, and let $\Delta^{\prime}$ denote the fundamental group of $\operatorname{Spec}\left(R^{\prime}\right) \times_{\operatorname{Spec}\left(R_{\bar{V}}\right)}$ $\operatorname{Spec}\left(R_{\bar{K}}^{o}\right)$. Then there is a canonical map $\Delta^{\prime} \rightarrow \Delta$ and it suffices to prove that $\Delta^{\prime} \rightarrow \Delta_{\infty}$ is surjective. This is equivalent to showing that for any collections of nonnegative integers $\underline{a}=\left(a_{1}, \ldots, a_{d}\right)$ the ring

$$
R_{\underline{a}}:=R^{\prime}\left[x_{1}, \ldots, x_{r}\right] /\left(x_{1}^{p^{a_{1}}}-t_{1}, \ldots, x_{r}^{p^{a_{d}}}-t_{d}\right)
$$

is an integral domain, where $t_{i} \in R^{\prime}$ denotes the image of $T_{i}$ in $R^{\prime}$ under 2.16.1. Since $R^{\prime}$ is a local ring, for this it suffices to show that the closed fiber $\operatorname{Spec}\left(R_{\underline{a}} / p R_{\underline{a}}\right)$ is connected. This is clear because $\operatorname{Spec}\left(R_{\underline{a}} / p R_{\underline{a}}\right) \rightarrow \operatorname{Spec}\left(R^{\prime} / p R^{\prime}\right)$ is a homeomorphism, and $\left(R^{\prime} / p R^{\prime}\right)_{\text {red }}$ is an integral domain being a localization of the ring $\left(R_{\bar{V}} / p R_{\bar{V}}\right)_{\text {red }}$ which is an integral domain by assumption.

Lemma 3.10. For any $\bar{R}$-module $M$ with continuous action of $\Delta$ and $i \geq 0$, the natural map $H^{i}\left(\Delta_{\infty}, M^{\Sigma}\right) \rightarrow H^{i}(\Delta, M)$ is an almost isomorphism.

Proof. Consider the spectral sequence

$$
E_{1}^{p q}=H^{p}\left(\Delta_{\infty}, H^{q}(\Sigma, M)\right) \Longrightarrow H^{p+q}(\Delta, M)
$$

arising from writing the functor $M \mapsto M^{\Delta}$ as the composite of functors

$$
\operatorname{Rep}_{\text {cts }}(\Delta) \stackrel{M \mapsto M^{\Sigma}}{\longrightarrow} \operatorname{Rep}_{\text {cts }}\left(\Delta_{\infty}\right) \stackrel{N_{\mapsto} N^{\Delta} \infty}{\longrightarrow} \text { (abelian groups). }
$$

The map in the Lemma is then the natural map $E_{1}^{i 0} \rightarrow H^{i}(\Delta, M)$. To prove the lemma it therefore suffices to show that for $q>0$ the group $H^{q}(\Sigma, M)$ is almost zero. For this note that

$$
H^{q}(\Sigma, M)=\underset{H}{\lim _{H}} H^{q}\left(\Sigma / H, M^{H}\right),
$$

where the limit is taken over normal subgroups $H \subset \Sigma$ of finite index, so it suffices to prove that each $H^{q}\left(\Sigma / H, M^{H}\right)$ is almost zero. Let $R_{\infty} \rightarrow S_{H}$ be the finite extension corresponding to $H$. Then by 2.17 the ring $S_{H}$ is almost étale and Galois over $R_{\infty}$. The result therefore follows from 3.4 .

3.11. Let $r>0$ be an integer. We can use the above results to (almost) compute the cohomology groups $H^{*}\left(\Delta, \bar{R} / p^{r} \bar{R}\right)$.

For this note that the natural map

$$
R_{\infty} / p^{r} R_{\infty} \rightarrow\left(\bar{R} / p^{r} \bar{R}\right)^{\Sigma}
$$


is an almost isomorphism. Inded this map becomes an almost isomorphism after applying $\otimes_{R_{\infty}} \bar{R}$ by 3.4 , and since $R_{\infty} \rightarrow \bar{R}$ is almost faithfully flat $(3.8)$, this implies that 3.11 .1 is an almost isomorphism. Using 3.10 it follows that the natural map

$$
H^{*}\left(\Delta_{\infty}, R_{\infty} / p^{r} R_{\infty}\right) \rightarrow H^{*}\left(\Delta, \bar{R} / p^{r} \bar{R}\right)
$$

is an almost isomorphism. It therefore suffices to compute $H^{*}\left(\Delta_{\infty}, R_{\infty} / p^{r} R_{\infty}\right)$.

Lemma 3.12. let $S \rightarrow S^{\prime}$ be a flat ring homomorphism, and let $M$ be an $S-$ module with continuous action of a profinite group $G$ over $S$. Then the natural map

$$
H^{*}(G, M) \otimes_{S} S^{\prime} \rightarrow H^{*}\left(G, M \otimes_{S} S^{\prime}\right)
$$

is an isomorphism.

Proof. Since $S \rightarrow S^{\prime}$ is flat, we have

$$
\left(M \otimes_{S} S^{\prime}\right)^{H}=M^{H} \otimes_{S} S^{\prime}
$$

for any subgroup $H \subset G$. By the definition of continuous group cohomology, we have

$$
H^{i}(G, M)=\underset{H \subset G}{\lim _{\vec{C} G}} H^{i}\left(G / H, M^{H}\right), \quad H^{i}\left(G, M \otimes_{S} S^{\prime}\right)=\underset{H \subset G}{\lim _{H}}\left(G / H, M^{H} \otimes_{S} S^{\prime}\right)
$$

where the limit is taken over normal subgroups $H \subset G$ of finite index. This therefore reduces the proof to the case when $G$ is finite.

Let $P_{\bullet} \rightarrow \mathbb{Z}$ denote the canonical resolution of $\mathbb{Z}$ defined in [43, VII, §3]. Recall that $P_{j}$ is isomorphic to $\mathbb{Z}^{G^{j+1}}$. Then by loc. cit. the cohomology groups $H^{i}(G, M)$ are equal to the the cohomology groups of $\operatorname{Hom}\left(P_{\bullet}, M\right)$ and similarly for $H^{i}\left(G, M \otimes_{S} S^{\prime}\right)$. The lemma then follows by observing that the natural map

$$
\operatorname{Hom}\left(P_{\bullet}, M\right) \otimes_{S} S^{\prime} \rightarrow \operatorname{Hom}\left(P_{\bullet}, M \otimes_{S} S^{\prime}\right)
$$

is an isomorphism since each $P_{j}$ is a finitely generated free module.

3.13. It follows that

$$
H^{*}\left(\Delta_{\infty}, R_{\infty} / p^{r} R_{\infty}\right) \simeq H^{*}\left(\Delta_{\infty}, S_{\infty} / p^{r} S_{\infty}\right) \otimes_{S_{\bar{V}}} R_{\bar{V}}
$$

where $S=V\left[T_{1}, \ldots, T_{d}\right]$. Now the Galois cohomology of $S_{\infty} / p^{r} S_{\infty}$ can be computed directly as follows. For positive numbers $\underline{a}=\left(a_{1}, \ldots, a_{d}\right)$ lying in $\mathbb{Z}[1 / p]$ we have a well-defined element $T^{\underline{a}}=T^{a_{1}} \cdots T^{a_{d}} \in \bar{S}_{\infty}$. Let $\mu_{\underline{a}}: \mathbb{Z}_{p}(1)^{d} \rightarrow \mu_{p^{\infty}}$ be the character giving the action on $T^{\underline{a}}$. If we consider an element $\lim _{n}\left(\zeta_{1, n}, \ldots, \zeta_{d, n}\right) \in$ $\mathbb{Z}_{p}(1)^{d}$, where $\zeta_{i, n}$ is an $n$-th root of unity, and if we write $a_{i}=b_{i} / p^{m}$ for some $m$, then $\mu_{\underline{a}}$ sends $\lim _{n}\left(\zeta_{1, n}, \ldots, \zeta_{d, n}\right)$ to $\zeta_{1, m}^{b_{1}} \cdots \zeta_{d, m}^{b_{d}}$. As in 3.5 let $L_{\mu_{\underline{a}}}$ denote the corresponding rank 1 free $\left(\bar{V} / p^{r} \bar{V}\right)$-module with continuous $\Delta_{\infty}$-action. Then as an $S$-module with continuous $\Delta_{\infty}$-action, we have

$$
S_{\infty}=\left(\oplus_{\underline{a}, 0 \leq a_{i}<1 \text { for all } i} L_{\mu_{\underline{a}}}\right) \otimes_{\bar{V}} S_{\bar{V}} .
$$

Using 3.12 again we have

$$
H^{*}\left(\Delta_{\infty}, S_{\infty} / p^{r} S_{\infty}\right) \simeq H^{*}\left(\Delta_{\infty}, \oplus_{\underline{a}} L_{\mu_{\underline{a}}}\right) \otimes_{\bar{V}} S_{\bar{V}} .
$$

Consider the decomposition

$$
\oplus_{\underline{a}} L_{\mu_{\underline{a}}}=L_{\mu_{0}} \oplus\left(\oplus_{\underline{a} \neq \underline{0}} L_{\mu_{\underline{a}}}\right) .
$$


By $3.5(\mathrm{i}))$ we have an isomorphism

$$
H^{*}\left(\Delta_{\infty}, L_{\mu_{0}}\right) \simeq \dot{\bigwedge}\left(\bar{V} / p^{r} \bar{V}\right)^{d}(-1)
$$

and by 3.5 (ii)) for any $\alpha \in \Lambda_{+}$the cohomology

$$
H^{*}\left(\Delta_{\infty}, L_{\mu_{\underline{a}}}\right)
$$

is annihilated by $\mathfrak{m}^{\alpha}$ for all but finitely many $\underline{a}$. From this we conclude the following:

Corollary 3.14. The $R_{\bar{V}}$-module $H^{*}\left(\Delta, \bar{R} / p^{r} \bar{V}\right)$ is almost finitely presented, $H^{i}\left(\Delta, \bar{R} / p^{r} \bar{R}\right)$ is almost zero for $i>d$, and for every $i$ we have an almost isomorphism

$$
H^{i}\left(\Delta, \bar{R} / p^{r} \bar{R}\right) \simeq\left(R_{\bar{V}} \otimes \bigwedge^{i}\left(\bar{V} / p^{r} \bar{V}\right)^{d}(-1)\right) \oplus N
$$

where $N$ is annihilated by $\zeta-1$, for a primitive $p$-th root of unity $\zeta$.

3.15. Let $J \subset R$ be the ideal $\left(T_{1} \cdots T_{d}\right)$, and let $\bar{J} \subset \bar{R}$ be the ideal of elements mapping to nilpotent elements in $\bar{R} /\left(T_{1} \cdots T_{d}\right)$. Then we can also use the above techniques to (almost) compute $H^{*}\left(\Delta, \bar{J} / p^{r} \bar{J}\right)$. Let $J_{\infty}^{(S)} \subset S_{\infty}$ be the ideal generated by elements $T_{1}^{a_{1}} \cdots T_{d}^{a_{d}}$ with $a_{i} \in \mathbb{Z}[1 / p]$ and $a_{i}>0$ for all $i$, and let $J_{\infty} \subset R_{\infty}$ denote the ideal $J_{\infty}^{(S)} \cdot R_{\infty}$. Note the following about these ideals:

(i) We have $J_{\infty}=\bar{J} \cap R_{\infty}$. Therefore by 3.4 the natural map

$$
J_{\infty} \otimes_{R_{\infty}} \bar{R} \rightarrow \bar{J}
$$

is an almost isomorphism.

(ii) Let $A_{\infty}$ denote the quotient $R_{\infty} / J_{\infty}$. Note that $A_{\infty}=R_{\infty} \otimes_{S_{\infty}}\left(S_{\infty} / J_{\infty}^{(S)}\right)$, and therefore is étale over $S_{\infty} / J_{\infty}^{(S)}$. It follows that $A_{\infty}$ is $p$-torsion free, since $S_{\infty} / J_{\infty}^{(S)} \simeq \bar{V}$. In particular, the sequence

$$
0 \rightarrow J_{\infty} / p^{r} J_{\infty} \rightarrow R_{\infty} / p^{r} R_{\infty} \rightarrow A_{\infty} / p^{r} A_{\infty} \rightarrow 0
$$

is exact.

(iii) We have a commutative diagram

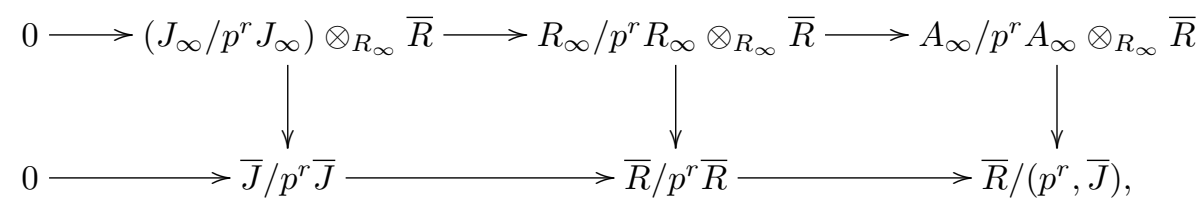

where the middle vertical arrow is an almost isomorphism, and the left vertical arrow is almost surjective. It follows that the natural map

$$
\left(J_{\infty} / p^{r} J_{\infty}\right) \otimes_{R_{\infty}} \bar{R} \rightarrow \bar{J} / p^{r} \bar{J}
$$

is an almost isomorphism. As in 3.11 this implies that the natural map

$$
J_{\infty} / p^{r} J_{\infty} \rightarrow\left(\bar{J} / p^{r} \bar{J}\right)^{\Sigma}
$$

is also an almost isomorphism.

Using these observations, 3.10 and 3.12 the group $H^{*}\left(\Delta, \bar{J} / p^{r} \bar{J}\right)$ is almost isomorphic to

$$
H^{*}\left(\Delta_{\infty}, J_{\infty}^{(S)} / p^{r} J_{\infty}^{(S)}\right) \otimes_{S_{\bar{V}}} R_{\bar{V}} .
$$

Going through the last step in the above proof one obtains: 
Proposition 3.16. The $R_{\bar{V}}$-module $H^{*}\left(\Delta, \bar{J} / p^{r} \bar{J}\right)$ is almost finitely presented, $H^{i}\left(\Delta, \bar{J} / p^{r} \bar{J}\right)$ is almost zero for $i>d$, and for every $i$ we have an almost isomorphism

$$
\left.H^{i}\left(\Delta, \bar{J} / p^{r} \bar{J}\right) \simeq J \otimes \bigwedge^{i}\left(\bar{V} / p^{r} \bar{V}\right)^{d}(-1)\right) \oplus N
$$

where $N$ is annihilated by $\zeta-1$, for a primitive $p$-th root of unity $\zeta$.

3.17. In particular, projection onto the first factor of $H^{d}\left(\Delta, \bar{J} / p^{r} \bar{J}\right)$ defines a trace map

$$
\operatorname{tr}: H^{d}(\Delta, \bar{J}) \rightarrow J \otimes_{V} \bar{V} / p^{r} \bar{V}(-d) .
$$

This can be described more canonically as follows. If we write

$$
H^{d}\left(\Delta, \bar{J} / p^{r} \bar{J}\right) \simeq J \otimes\left(\bar{V} / p^{r} \bar{V}\right)(-d) \oplus N_{r}
$$

where $N_{r}$ is annihilated by $\zeta-1$, then as $r$ varies we get a compatible collection of maps

$$
\operatorname{tr}_{r}: H^{d}(\Delta, \bar{J}) \rightarrow J \otimes_{V} \bar{V} / p^{r} \bar{V}(-d)
$$

such that the composite map

$$
J \otimes_{V} \bar{V} / p^{r} \bar{V}(-d) \rightarrow H^{d}(\Delta, \bar{J}) \rightarrow J \otimes_{V} \bar{V} / p^{r} \bar{V}(-d)
$$

is the identity. Since each $N_{r}$ is annihilated by $\zeta-1$, there is a unique such compatible collection of maps $\operatorname{tr}_{r}$.

3.18. Let $L$ be a representation of $\Delta$ on a finitely generated free $\mathbb{Z} / p^{r}$-module.

Proposition 3.19. (i) The $R_{\infty}$-module $(L \otimes \bar{R})^{\Sigma}$-module is almost finitely presented.

(ii) For every $i$ the cohomology group $H^{i}(\Delta, L \otimes \bar{R})$ is almost finitely presented over $R_{\bar{V}}$, and these groups are almost zero for $i>d$.

Proof. For (i), note that by the second statement in 3.4 the natural map

$$
(L \otimes \bar{R})^{\Sigma} \otimes_{R_{\infty}} \bar{R} \rightarrow L \otimes \bar{R}
$$

is an almost isomorphism. From this it follows that $(L \otimes \bar{R})^{\Sigma}$ is almost finitely presented since $\bar{R} / R_{\infty}$ is almost faithfully flat by 3.8 (see [21, 3.4.1] for descent in the almost category).

For (ii), let $R_{n} \subset R_{\infty}$ denote the subring generated by $\bar{V}$ and $T_{i}^{1 / p^{n}}$ (notation as in 2.16. Let $\Delta_{n, \infty} \subset \Delta_{\infty}$ denote the subgroup of elements acting trivially on $R_{n}$, so the quotient $\Delta_{\infty} / \Delta_{n, \infty}$ is finite. Since $(L \otimes \bar{R})^{\Sigma}$ is almost finitely presented over $R_{\infty}$, for every $\alpha \in \Lambda_{+}$there exists an integer $n$ and a finitely presented free module $M_{\alpha}$ over $R_{n}$ with a morphism

$$
M_{\alpha} \otimes_{R_{n}} R_{\infty} \rightarrow(L \otimes \bar{R})^{\Sigma}
$$

whose cokernel is annihilated by $\mathfrak{m}^{\alpha}$. By possibly increasing $n$, we may assume that the map is $\Delta_{n, \infty}$-invariant, where $\Delta_{n, \infty}$ acts trivially on $M_{\alpha}$. Applying induction from $\Delta_{n, \infty}$ to $\Delta_{\infty}$ we obtain a morphism of $\Delta_{\infty}$-modules

$$
N_{\alpha} \otimes_{R_{n}} R_{\infty} \rightarrow(L \otimes \bar{R})^{\Sigma}
$$

where $N_{\alpha}$ is a finitely presented free $R_{n}$-module, the cokernel is annihilated by $\mathfrak{m}_{\alpha}$, and $\Delta_{n, \infty}$ acts trivially on $N_{\alpha}$. The kernel of this map is again almost finitely presented over $R_{\infty}$. Repeating the argument (and possibly replacing $n$ by a bigger 
integer), we find that there exists a finitely presented $R_{n}$-module $M_{\alpha}$ with semilinear $\Delta_{\infty}$-action (which restricts to the trivial action of $\Delta_{n, \infty}$ ) and a morphism

$$
M_{\alpha} \otimes_{R_{n}} R_{\infty} \rightarrow(L \otimes \bar{R})^{\Sigma}
$$

whose kernel and cokernel is annihilated by $\mathfrak{m}^{\alpha}$.

By the same argument used in 3.13 , we can then find a decomposition as a $R_{n}$-module with $\Delta_{n, \infty}$-action

$$
M_{\alpha} \otimes_{R_{n}} R_{\infty} \simeq M_{\alpha} \oplus H_{\alpha},
$$

where $R \Gamma_{\Delta_{n, \infty}}\left(H_{\alpha}\right)$ is annihilated by $\zeta_{p^{n}}-1$ for some primitive $p^{n}$-th root of unity $\zeta_{p^{n}}$. After possibly increasing $n$, we may further assume that the $p$-adic valuation of $\zeta_{p^{n}}-1$ is less than $\alpha$. In fact, we have a decomposition

$$
M_{\alpha} \otimes_{R_{n}} R_{\infty} \simeq \oplus_{\underline{a}} M_{\alpha} \otimes_{R_{n}} L_{\mu_{\underline{a}}},
$$

where each $L_{\mu_{\underline{a}}}$ is a rank 1 representation over $R_{n}$, and $H_{\alpha}$ is obtained by taking the factors corresponding to $L_{\mu_{\underline{a}}}$ with $\underline{a}$ nontrivial. Since $\Delta_{\infty}$ is commutative, the decomposition 3.19 .1 is even $\Delta_{\infty}$-invariant. Since

$$
R \Gamma_{\Delta_{\infty}}\left(H_{\alpha}\right)=R \Gamma_{\Delta_{\infty} / \Delta_{n, \infty}} R \Gamma_{\Delta_{n, \infty}}\left(H_{\alpha}\right),
$$

this implies that $R \Gamma_{\Delta_{\infty}}\left(H_{\alpha}\right)$ is annihilated by $\mathfrak{m}^{\alpha}$. From this we conclude that up to $\mathfrak{m}^{\alpha}$-torsion, the cohomology $R \Gamma_{\Delta}(L \otimes \bar{R})$ is represented by the Koszul complex of the $\Delta_{\infty}$-representation $M_{\alpha}$, after choosing a basis for $\Delta_{\infty}$. This implies (ii).

REMARK 3.20. By a similar argument, the cohomology groups $H^{i}(\Delta, L \otimes \bar{J})$ are almost finitely presented, and zero for $i>d$.

3.21. The method used to prove 3.19 can also be used to study the natural duality morphism

$$
R \Gamma_{\Delta}(L \otimes \bar{R}) \rightarrow R \operatorname{Hom}\left(R \Gamma_{\Delta}\left(L^{*} \otimes \bar{J}\right), J \otimes_{V} \bar{V} / p^{r} \bar{V}(-d)[-d]\right)
$$

defined in $\widetilde{D}(\bar{R})$. Here $L^{*}$ denotes the dual $\operatorname{Hom}\left(L, \mathbb{Z} / p^{r}\right)$ and

$$
\Gamma_{\Delta}: \operatorname{Rep}^{\text {cts }}(\Delta) \rightarrow(\text { Groups })
$$

is the functor taking $\Delta$-invariants.

With notation as in the proof, we compute

$$
R \Gamma_{\Delta}\left(L^{*} \otimes \bar{J}\right)
$$

as follows. Note first of all that since the natural maps

$$
(L \otimes \bar{R})^{\Sigma} \otimes_{R_{\infty}} \bar{R} \rightarrow L \otimes \bar{R}, \quad J_{\infty} \otimes_{R_{\infty}} \bar{R} \rightarrow \bar{R}
$$

are almost isomorphisms, $(L \otimes \bar{R})^{\Sigma}$ is almost finitely presented, and $\bar{R} / R_{\infty}$ is almost faithfully flat, the natural map

$$
\operatorname{Hom}_{R_{\infty}}\left((L \otimes \bar{R})^{\Sigma}, J_{\infty}\right) \otimes_{R_{\infty}} \bar{R} \rightarrow \operatorname{Hom}_{\bar{R}}(L \otimes \bar{R}, \bar{J})
$$

is an almost isomorphism. It follows that the natural map

$$
\operatorname{Hom}_{R_{\infty}}\left((L \otimes \bar{R})^{\Sigma}, J_{\infty}\right) \rightarrow\left(L^{*} \otimes \bar{J}\right)^{\Sigma}
$$

becomes an almost isomorphism after tensoring with $\bar{R}$, and hence is already an almost isomorphism. On the other hand, since $(L \otimes \bar{R})^{\Sigma}$ is almost finitely presented, we have an almost isomorphism

$$
\operatorname{Hom}_{R_{\infty}}\left((L \otimes \bar{R})^{\Sigma}, J_{\infty}\right) \simeq \operatorname{Hom}_{R_{\infty}}\left((L \otimes \bar{R})^{\Sigma}, R_{\infty}\right) \otimes J_{\infty} .
$$


Now up to $\mathfrak{m}^{\alpha}$-torsion, this is equal to

$$
\operatorname{Hom}_{R_{n}}\left(M_{\alpha}, R_{n}\right) \otimes_{R_{n}} J_{\infty} .
$$

Now decomposing $J_{\infty}$ into $J_{n}:=J_{\infty} \cap R_{n}$ plus a part whose cohomology is annihilated by $\mathfrak{m}^{\alpha}$, we find that up to $\mathfrak{m}^{\alpha}$-torsion the cohomology

$$
R \Gamma_{\Delta}\left(L^{*} \otimes \bar{J}\right)
$$

is given by

$$
R \Gamma_{\Delta_{\infty}}\left(\operatorname{Hom}_{R_{n}}\left(M_{\alpha}, R_{n}\right) \otimes_{R_{n}} J_{n}\right) .
$$

Since $R_{n} / R_{\bar{V}}$ is a finite flat covering there is a trace map

$$
\operatorname{tr}: R_{n} \rightarrow R_{\bar{V}}
$$

which sends $J_{n}$ to $J_{\bar{V}}$. For a collection of integers $\left(a_{1}, \ldots, a_{d}\right)$, this trace map sends

$$
T_{1}^{a_{1} / p^{n}} \cdots T_{d}^{a_{d} / p^{n}} \mapsto\left(\sum_{\underline{i}=\left(i_{1}, \ldots, i_{d}\right)} \zeta^{i_{1} a_{1}} \cdots \zeta^{i_{d} a_{d}}\right) T_{1}^{a_{1} / p^{n}} \cdots T_{d}^{a_{d} / p^{n}},
$$

where $\zeta$ is a primitive $p^{n}$-th root of unity, and the right sum is taken over $\underline{i} \in$ $\left(\mathbb{Z} / p^{n}\right)^{d}$. Since we have

$$
\left(\sum_{\underline{i}=\left(i_{1}, \ldots, i_{d}\right)} \zeta^{i_{1} a_{1}} \cdots \zeta^{i_{d} a_{d}}\right)=\prod_{j=1}^{d}\left(1+\zeta^{a_{j}}+\left(\zeta^{a_{j}}\right)^{2}+\cdots+\left(\zeta^{a_{j}}\right)^{p^{n}-1}\right),
$$

it follows that

$$
\operatorname{tr}\left(T_{1}^{a_{1} / p^{n}} \cdots T_{d}^{a_{d} / p^{n}}\right)= \begin{cases}\operatorname{deg}\left(R_{n} / R_{\bar{V}}\right) \cdot T_{1}^{a_{1} / p^{n}} \cdots T_{d}^{a_{d} / p^{n}} & \text { if } p^{n} \mid a_{j} \text { for all } j \\ 0 & \text { otherwise. }\end{cases}
$$

We therefore can describe the projection onto the $\mu_{0}$-component (notation as in 3.13 as the map

$$
\operatorname{tr}_{n}:=\frac{1}{\operatorname{deg}\left(R_{n} / R_{\bar{V}}\right)} \cdot \operatorname{tr}: R_{n} \rightarrow R_{\bar{V}}
$$

As before this map sends $J_{n}$ to $J_{\bar{V}}$, and in fact it follows from this description that the map

$$
J_{n} \rightarrow \operatorname{Hom}_{R_{\bar{V}}}\left(R_{n}, J_{\bar{V}}\right), \quad x \mapsto\left(y \mapsto \operatorname{tr}_{n}(x y)\right)
$$

is an isomorphism of free $R_{n}$-modules of rank 1. We therefore obtain an isomorphism between 3.21 .3 and

$$
R \Gamma_{\Delta_{\infty}}\left(\operatorname{Hom}_{R_{n}}\left(M_{\alpha}, R_{n}\right) \otimes_{R_{n}} \operatorname{Hom}_{R_{\bar{V}}}\left(R_{n}, J_{\bar{V}}\right)\right) .
$$

REMARK 3.22. Note that the trace maps $\operatorname{tr}_{n}$ are compatible, and induce a trace map

$$
\operatorname{tr}_{\infty}: R_{\infty} \rightarrow R_{\bar{V}}
$$

which again is just the projection onto the $\mu_{0}$-component.

Lemma 3.23. For any finitely presented $R_{n}$-module $W$, the natural map

$$
\operatorname{Hom}_{R_{n}}\left(W, R_{n}\right) \otimes_{R_{n}} \operatorname{Hom}_{R_{\bar{V}}}\left(R_{n}, J_{\bar{V}}\right) \rightarrow \operatorname{Hom}_{R_{\bar{V}}}\left(W, J_{\bar{V}}\right)
$$

is an isomorphism. 
Proof. If $W$ is a finitely generated free module, the result is immediate. For the general case, write $W$ is a cokernel

$$
W=\operatorname{Coker}\left(F_{1} \rightarrow F_{2}\right)
$$

of a morphism between finitely presented free $R_{n}$-modules. We then have an exact sequence

$$
0 \rightarrow \operatorname{Hom}_{R_{n}}\left(W, R_{n}\right) \rightarrow \operatorname{Hom}_{R_{n}}\left(F_{2}, R_{n}\right) \rightarrow \operatorname{Hom}_{R_{n}}\left(F_{1}, R_{n}\right) .
$$

Tensoring this sequence with $\operatorname{Hom}_{R_{\bar{V}}}\left(R_{n}, J_{\bar{V}}\right)$ and comparing it with

$$
0 \rightarrow \operatorname{Hom}_{R}\left(W, J_{\bar{V}}\right) \rightarrow \operatorname{Hom}_{R}\left(F_{2}, J_{\bar{V}}\right) \rightarrow \operatorname{Hom}\left(F_{1}, J_{\bar{V}}\right)
$$

then gives the result.

3.24. We therefore obtain that up to $\mathfrak{m}^{\alpha}$-torsion, $R \Gamma_{\Delta}\left(L^{*} \otimes \bar{J}\right)$ is equal to

$$
R \Gamma_{\Delta_{\infty}}\left(\operatorname{Hom}_{R_{\bar{V}}}\left(M_{\alpha}, J_{\bar{V}}\right)\right) \text {. }
$$

By Koszul duality 3.6.2, this induces an isomorphism up to $\mathfrak{m}^{\alpha}$-torsion

$$
\left.R \operatorname{Hom}\left(R \Gamma_{\Delta}(L \otimes \bar{R}), J_{\bar{V}}\right) \simeq R \Gamma_{\Delta}\left(L^{*}, \bar{J}\right)\right)[d] .
$$

We leave to the reader the verification that up to $\mathfrak{m}^{\alpha}$-torsion this map induces the map 3.21.1, thereby proving the following theorem:

THEOREM 3.25. The morphism 3.21.1 is an almost isomorphism.

3.26. More generally if $E \subset\{1, \ldots, n\}$ is a subset and $D_{E} \subset D$ the union of the divisors $D_{i}$ with $i \in E$, then we define $\bar{J}_{E} \subset \bar{R}$ to be the ideal of elements mapping to nilpotent elements in the coordinate ring of $D_{E} \times_{X} \operatorname{Spec}(\bar{R})$.

If $F \subset\{1, \ldots, n\}$ denotes the complement of $E$, then there is a canonical map $\bar{J}_{F} \otimes \bar{J}_{E} \rightarrow \bar{J}$ which induces for any representation $L$ of $\Delta$ a canonical map

$$
R \Gamma_{\Delta}\left(L \otimes \bar{J}_{E}\right) \otimes^{\mathbb{L}} R \Gamma_{\Delta}\left(L^{*} \otimes \bar{J}_{F}\right) \rightarrow R \Gamma_{\Delta}(\bar{J}) .
$$

Composing with the trace map we obtain a morphism

$$
R \Gamma_{\Delta}\left(L \otimes \bar{J}_{E}\right) \rightarrow R \operatorname{Hom}\left(R \Gamma_{\Delta}\left(L^{*} \otimes \bar{J}_{F}\right), J \otimes_{V} \bar{V} / p^{r} \bar{V}(-d)[-d]\right) .
$$

A local calculation as in the proof of 3.25 shows that this is an almost isomorphism.

\section{Logarithmic geometry}

4.1. In order to globalize the results of the preceding section, it is useful to introduce a bit of log geometry in the sense of Fontaine, Illusie, and Kato [29.

4.2. Let $S$ be a scheme which we view as a prelog scheme with the trivial log structure, and let $\left(X, M_{X}\right) \rightarrow S$ be a morphism of prelog schemes (we usually write $\alpha: M_{X} \rightarrow \mathscr{O}_{X}$ for the map in the definition of a prelog structure). Let $E$ be a quasi-coherent $\mathscr{O}_{X}$-module.

Definition $4.3([38,1.1 .2])$. A log derivation of $\left(\mathscr{O}_{X}, M_{X}\right)$ with values in $E$ is a pair $\partial=(D, \delta)$, where $\delta: \mathscr{O}_{X} \rightarrow E$ is a derivation and $D: M_{X} \rightarrow E$ is an additive map such that for every local section $m \in M_{X}$ we have $\alpha(m) D(m)=\delta(\alpha(m)$ ) (so $D(m)$ should be viewed as " $d \log (\alpha(m))$ ").

Remark 4.4. Note that the map $D$ and the equation $\alpha(m) D(m)=\delta(\alpha(m))$ determines the derivation $\delta$ since a derivation is determined by its value on the sections of $\mathscr{O}_{X}^{*}$. 
4.5. If $\Omega_{\left(X, M_{X}\right) / S}^{1}$ denotes the logarithmic differentials [29, 1.7], then there is a universal $\log$ derivation (see for example [38, 1.1])

$$
d: \mathscr{O}_{X} \rightarrow \Omega_{\left(X, M_{X}\right) / S}^{1}, \quad D: M_{X} \rightarrow \Omega_{\left(X, M_{X}\right) / S}^{1} .
$$

In other words, if $E$ is a quasi-coherent $\mathscr{O}_{X}$-module then there is a canonical bijection

$\left(\log\right.$ derivations of $\left(\mathscr{O}_{X}, M_{X}\right)$ with values in $\left.E\right) \simeq \operatorname{Hom}_{\mathscr{O}_{X}}\left(\Omega_{\left(X, M_{X}\right) / S}^{1}, E\right)$.

Note in particular that if $X$ is affine and $P \rightarrow \Gamma\left(X, M_{X}\right)$ is a chart, then since $\Omega_{(X, P) / S}^{1} \simeq \Omega_{\left(X, M_{X}\right) / S}^{1}$ (this equality is explained in [29, 1.7]), to give a morphism $\Omega_{\left(X, M_{X}\right) / S}^{1} \rightarrow E$ is equivalent to giving an additive map $D: P \rightarrow \Gamma(X, E)$ and a derivation $\delta: \Gamma\left(X, \mathscr{O}_{X}\right) \rightarrow \Gamma(X, E)$ so that for all $p \in P$ we have $\alpha(p) D(p)=$ $\delta(\alpha(p))$.

4.6. Now let the notation be as in 3.11, let $X=\operatorname{Spec}(R)$, let $M_{X}$ be the log structure defined by the divisor $\operatorname{Spec}(R)-\operatorname{Spec}\left(R^{o}\right)$, let $M_{R}$ denote $\Gamma\left(X, M_{X}\right)$, and let $\Omega_{\left(R, M_{R}\right) / V}^{1}$ denote the global sections of $\Omega_{\left(X, M_{X}\right) / \operatorname{Spec}(V)}^{1}$. Since there exists a morphism as in 2.16.1 the image of $M_{R}$ under the map $d \log$ generates $\Omega_{\left(R, M_{R}\right) / V}^{1}$, and therefore for any $R$-module $E$, a homomorphism $\Omega_{\left(R, M_{R}\right) / V}^{1} \rightarrow E$ is determined by an additive map

$$
D: M_{R} \rightarrow E .
$$

As in 3.11 let $r$ be an integer. We now construct a morphism

$$
c: \Omega_{\left(R, M_{R}\right) / V}^{1} \rightarrow H^{1}\left(\Delta, \bar{R} / p^{r} \bar{R}(1)\right)
$$

depending only on $\operatorname{Spec}(R)$ with its log structure. For this we first construct an additive map

$$
D: M_{R} \rightarrow H^{1}\left(\Delta, \bar{R} / p^{r} \bar{R}(1)\right) .
$$

Note that the map $M_{R} \rightarrow R$ is an inclusion. For every $s \geq 0$ let $M_{s} \subset \bar{R}$ denote the set of elements $f \in \bar{R}$ with $f^{p^{s}} \in M_{R}$. There is then a natural exact sequence in the category of integral monoids

$$
0 \longrightarrow \mu_{p^{s}} \longrightarrow M_{s} \stackrel{\times p^{s}}{\longrightarrow} M_{R} \longrightarrow 0 .
$$

Raising to the $p$-th power induces a surjection $M_{s+1} \rightarrow M_{s}$. Let $E:=\varliminf_{\lim } M_{s}$ denote the projective limit, and note that the sequences 4.6 .4 induce an exact sequence (again in the category of integral monoids)

$$
0 \longrightarrow \mathbb{Z}_{p}(1) \longrightarrow M_{R} \longrightarrow 0 \text {. }
$$

The action of $\Delta$ on $\bar{R}$ induces an action of $\Delta$ on $E$ which makes this an extension in the category of monoids with continuous $\Delta$-action. The sequence 4.6 .5 therefore induces an additive map

$$
M_{R} \rightarrow H^{1}\left(\Delta, \mathbb{Z}_{p}(1)\right) .
$$

Composing with the natural map $H^{1}\left(\Delta, \mathbb{Z}_{p}(1)\right) \rightarrow H^{1}\left(\Delta, \bar{R} / p^{s} \bar{R}(1)\right)$ we obtain a map

$$
D: M_{R} \rightarrow H^{1}\left(\Delta, \bar{R} / p^{s} \bar{R}(1)\right)
$$


TheOREM 4.7. The map $M_{R}^{g p} \otimes_{\mathbb{Z}} R / p^{s} \bar{R} \rightarrow H^{1}\left(\Delta, R / p^{s} \bar{R}(1)\right)$ induced by 4.6 .7 descends to $\Omega_{\left(R, M_{R}\right) / V}^{1}$, and the resulting map

$$
\Omega_{\left(R, M_{R}\right) / V}^{1} \otimes_{R} R_{\bar{V}} / p^{s} R_{\bar{V}}(-1) \rightarrow H^{1}\left(\Delta, \bar{R} / p^{s} \bar{R}\right)
$$

induces an almost morphism

$$
\dot{\bigwedge}\left(\Omega_{\left(R, M_{R}\right) / V}^{1} \otimes_{R} R_{\bar{V}} / p^{s} R_{\bar{V}}(-1)\right) \rightarrow H^{*}\left(\Delta, \bar{R} / p^{s} \bar{R}\right)
$$

Proof. For $f \in R^{*}$, let $T_{f}$ denote the $\mathbb{Z}_{p}(1)$-torsor with $\Delta$-action inducing $c(f) \in H^{1}\left(\Delta, \bar{R} / p^{s} \bar{R}(1)\right)$. The torsor $T_{f}$ is equal to the projective limit $T_{f}=$ $\varliminf_{f, n} T_{f, n}$, where $T_{f, n}$ is the set of elements $g \in \bar{R}^{*}$ such that $g^{p^{n}}=f$ and the map $\overleftarrow{T}_{f, n+1} \rightarrow T_{f, n}$ sends $g$ to $g^{p}$.

Lemma 4.8. The map $\left.c\right|_{R^{*}}: R^{*} \rightarrow H^{1}\left(\Delta, \bar{R} / p^{s} \bar{R}(1)\right)$ factors through the image of $d \log : R^{*} \rightarrow \Omega_{\left(R, M_{R}\right) / V}^{1}$.

Proof. It suffices to show that if $d \log (f)=d \log (g)$ then the torsors with $\Delta$-action $T_{f}$ and $T_{g}$ are isomorphic, or equivalently that the torsor with $\Delta$-action $T_{f g^{-1}} \simeq T_{f} \wedge T_{g}^{-1}$ is trivial. This is clear, for if $d \log (f)=d \log (g)$ then

$$
d \log \left(f g^{-1}\right)=d \log (f)-d \log (g)=0
$$

and hence $d\left(f g^{-1}\right)=0$. Since $\operatorname{Spec}\left(R \otimes_{V} K\right) \rightarrow \operatorname{Spec}(K)$ is formally étale this implies that $f g^{-1} \in R \cap K=V$. This in turn implies that the action of $\Delta$ on $T_{f g^{-1}}$ is trivial which proves the lemma.

For the proof of 4.7, consider first the case when $R=S:=V\left[T_{1}, \ldots, T_{d}\right]$. In this case $M_{R}=V^{*} \oplus \mathbb{N}^{d}$, where the $i$-th generator $e_{i} \in \mathbb{N}^{d}$ maps to the element $t_{i}$ in $S$. The class $c\left(e_{i}\right) \in H^{1}\left(\Delta, \bar{S} / p^{r} \bar{S}\right)$ is then given by the $\mathbb{Z}_{p}$-torsor of $p$-power roots of $t_{i}$ in $\bar{S}$. Note that the action of $\Delta$ on this torsor factors through $\Delta_{\infty}$. It follows that in this case the map $c$ factors through a map

$$
\tilde{c}: V^{*} \oplus \mathbb{N}^{d} \rightarrow H^{1}\left(\Delta_{\infty}, S_{\infty} / p^{r} S_{\infty}(1)\right) .
$$

Furthermore, $\tilde{c}\left(V^{*}\right)=0$ which proves that $\tilde{c}$ factors through $\Omega_{\left(S, M_{S}\right) / V}^{1}$. Chasing through the construction of the isomorphism

$$
\left(S_{\bar{V}} / p^{r} S_{\bar{V}}\right)^{d} \simeq H^{1}\left(\Delta_{\infty}, S_{\infty} / p^{r} S_{\infty}(1)\right)
$$

given in 3.11, one sees that the map $\tilde{c}$ induces an isomorphism

$$
\Omega_{\left(S, M_{S}\right) / V}^{1} \otimes_{S}\left(S_{\bar{V}} / p^{r} S_{\bar{V}}\right) \simeq\left(S_{\bar{V}} / p^{r} S_{\bar{V}}\right) \otimes_{\mathbb{Z}} \mathbb{Z}^{d} \rightarrow H^{1}\left(\Delta_{\infty}, S_{\infty} / p^{r} S_{\infty}(1)\right) .
$$

This proves the theorem in the case when $R=S$.

For the general case choose a formally étale map $S \rightarrow R$ as in 2.16.1.

Lemma 4.9. The natural map $R^{*} \oplus_{S^{*}} M_{S} \rightarrow M_{R}$ is surjective.

Proof. Let $t_{i} \in R$ be the image of $T_{i}$. Then to prove the lemma it suffices to show that any element $m \in M_{R} \subset R$ can be written as $u t_{1}^{a_{1}} \cdots t_{d}^{a_{d}}$ where $u \in R^{*}$. This is clear, for if $m \in M_{R}$ let $a_{i}$ denote the valuation of the image of $m$ in the local ring of the generic point of the divisor of $\operatorname{Spec}(R)$ lying over $\operatorname{Spec}\left(S /\left(T_{i}\right)\right) \subset \operatorname{Spec}(S)$ (if the inverse image is empty then set $a_{i}=1$ ). Since $R$ is regular, the elements $m$ and $t_{1}^{a_{1}} \cdots t_{d}^{a_{d}}$ must differ by a unit. 
By the discussion at the beginning of the proof the composite

$$
M_{S} \oplus_{S^{*}} R^{*} \longrightarrow M_{R} \stackrel{c}{\longrightarrow} H^{1}\left(\Delta, \bar{R} / p^{r} \bar{R}(1)\right)
$$

is equal to the composite

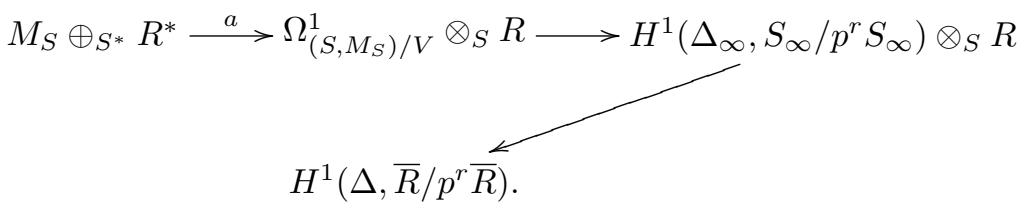

where the map $a$ is induced by the map $d \log : R^{*} \rightarrow \Omega_{\left(R, M_{R}\right) / V}^{1} \simeq \Omega_{\left(S, M_{S}\right) / V} \otimes_{S} R$. By the surjectivity in 4.9 this implies 4.7 .

Corollary 4.10. There is a canonical (i.e. depending only on $\operatorname{Spec}(R)$ with its boundary divisor) almost morphism, called the trace map,

$$
H^{d}\left(\Delta, \bar{J} / p^{r} \bar{J}\right) \rightarrow \Omega_{R / V}^{d} \otimes_{V} \bar{V} / p^{r} \bar{V}(-d) .
$$

Proof. This follows from 3.16, 4.7 and the observation that there is a canonical isomorphism $J \otimes \Omega_{\left(R, M_{R}\right) / V}^{1} \simeq \Omega_{R / V}^{1}$.

Restating 3.25 we obtain:

COROLLARY 4.11. The trace map induces a canonical isomorphism in $\widetilde{D}\left(R_{\bar{V}}\right)$

$$
R \Gamma_{\Delta}(L \otimes \bar{R}) \rightarrow R \operatorname{Hom}\left(R \Gamma_{\Delta}\left(L^{*} \otimes \bar{J}\right), \Omega_{R / V}^{d} \otimes_{V} \bar{V} / p^{r} \bar{V}(-d)[-d]\right)
$$

More generally, for a subset $E \subset\{1, \ldots, n\}$ with complementary set $F$ there is a canonical isomorphism in $\widetilde{D}(\bar{R})$

$$
R \Gamma_{\Delta}\left(L \otimes \bar{J}_{E}\right) \rightarrow R \operatorname{Hom}\left(R \Gamma_{\Delta}\left(L^{*} \otimes \bar{J}_{F}\right), \Omega_{R / V}^{d} \otimes_{V} \bar{V} / p^{r} \bar{V}(-d)[-d]\right)
$$

\section{Coverings by $K(\pi, 1)$ 's}

5.1. The key to globalizing the above computations is the notion of a $K(\pi, 1)$. If $X$ is a scheme, let $\operatorname{Fet}(X)$ denote the site whose underlying category is the category of finite étale morphisms $U \rightarrow X$, and whose coverings are surjective morphisms. We write $X_{\mathrm{Fet}}$ for the associated topos. The inclusion $\operatorname{Fet}(X) \subset \operatorname{Et}(X)$ induces a morphism of topoi

$$
\pi: X_{\mathrm{et}} \rightarrow X_{\mathrm{Fet}}
$$

If $X$ is connected and $\bar{x} \rightarrow X$ is a geometric point, then $X_{\mathrm{Fet}}$ is equivalent to the category of sets $F_{\bar{x}}$ with continuous action of the fundamental group $\pi_{1}(X, \bar{x})$ so in particular for an abelian sheaf $F$ the cohomology $H^{*}\left(X_{\mathrm{Fet}}, F\right)$ is isomorphic to group cohomology $H^{*}\left(\pi_{1}(X, \bar{x}), F_{\bar{x}}\right)$.

In general, $\pi^{*}$ identifies the category $X_{\text {Fet }}$ with the category of sheaves $F \in X_{\text {et }}$ for which $F$ is equal to the union of its locally constant subsheaves.

In what follows let $\Lambda$ denote $\mathbb{Z} /(n)$ for some integer $n$ invertible on $X$.

REMARK 5.2. It is always true that for a locally constant sheaf $L$ of $\Lambda$-modules on $X$ the natural map $H^{1}\left(X_{\mathrm{Fet}}, L\right) \rightarrow H^{1}\left(X_{\mathrm{et}}, \pi^{*} L\right)$ is an isomorphism. This follows from interpreting these groups as classifying extensions of $\Lambda$ by $L$ and noting that any such extension is also locally constant. 
Definition 5.3. A scheme $X$ is a $K(\pi, 1)$ if for integer $n$ invertible on $X$ and every locally constant sheaf $F$ of $\mathbb{Z} /(n)$-modules the natural map $F \rightarrow R \pi_{*} \pi^{*} F$ is an isomorphism.

THEOREM 5.4. Let $V$ be a discrete valuation ring with field of fractions $K$ of characteristic 0 and perfect residue field $k$. Let $X / V$ be a smooth scheme. Then for every point $x \in X$ in the closed fiber of $X$ there exists a Zariski open neighborhood $U \subset X$ of $x$ such that the geometric generic fiber $U_{\bar{K}}$ is a $K(\pi, 1)$. In particular, if $X / V$ is also proper then every point $x \in X$ is contained in an open subset $U \subset X$ such that $U_{\bar{K}}$ is a $K(\pi, 1)$.

Proof. By induction on the relative dimension $d$ of $X / V$. We can without loss of generality assume that $X$ is affine.

The case $d=1$ can be seen as follows. Choose an embedding $X \hookrightarrow \mathbb{P}_{V}$ into some projective space and let $\bar{X}$ be the normalization of the closure. The generic fiber $\bar{X}_{K}$ is then a normal proper 1-dimensional $K$-scheme whence a smooth proper curve. Since $\bar{X}_{K}-X_{K}$ is not empty, the cohomological dimension of $X_{\bar{K}}$ is 1 . This case therefore follows from 5.2 .

The case $d=1$ can also be relativized. As in [1, XI.3.1] define a morphism $f: Z \rightarrow W$ of $K$-schemes to be an elementary fibration if it extends to a proper smooth morphism $\bar{f}: \bar{Z} \rightarrow W$ such that the map $\bar{Z}-Z \rightarrow W$ (where $\bar{Z}-Z$ is given the reduced structure) is finite étale and surjective and for every geometric point $\bar{w} \rightarrow W$ the geometric fiber $\bar{Z}_{\bar{w}}$ is a smooth connected (and automatically proper) curve. Then $Z_{\bar{w}}$ is a smooth affine curve. We extend this notion slightly by defining a morphism $f: X \rightarrow Y$ of $V$-schemes to be an elementary fibration if the generic fiber $X_{K} \rightarrow Y_{K}$ is an elementary fibration.

Lemma 5.5. Let $f: Z \rightarrow W$ be an elementary fibration of smooth $K$-schemes. If $W$ is a $K(\pi, 1)$ then $Z$ is also a $K(\pi, 1)$.

Proof. We have to show that for any locally constant constructible sheaf $L$ on $Z$ and $i>0$ the sheaf $R^{i} \pi_{*} L$ on $Z_{\mathrm{Fet}}$ is zero. For this we may clearly replace $W$ by a finite étale cover, and hence making the base change $(\bar{Z}-Z) \rightarrow W$ we can assume that the complement $\bar{Z}-Z$ is given by a nonempty collection of sections $s: W \rightarrow \bar{Z}$. If $U \rightarrow Z$ is finite étale and surjective and $\bar{U}$ denotes the normalization of $\bar{Z}$ in $U$, then it follows from Abhyankar's lemma and the assumption $\operatorname{char}(K)=0$ that $\bar{U} \rightarrow W$ is also smooth and proper and that $\bar{U}-U \rightarrow W$ is finite étale and surjective. Replacing $Z$ by a finite étale covering where $L$ is trivial we may therefore assume that $L=\Lambda$. We may also base change to $\bar{K}$ and hence may assume that $K$ is algebraically closed. Furthermore, it suffices to show that for any cohomology class $\alpha \in H^{i}\left(Z_{\mathrm{et}}, \Lambda\right)$ there exists a finite étale surjective morphism $Z^{\prime} \rightarrow Z$ such that the pullback of $\alpha$ to $Z^{\prime}$ is trivial.

Next consider the Leray spectral sequence

$$
E_{2}^{i j}=H^{i}\left(W, R^{j} f_{*} \Lambda\right) \Longrightarrow H^{i+j}(Z, \Lambda) .
$$

Let $j: Z \hookrightarrow \bar{Z}$ and $i:(\bar{Z}-Z) \hookrightarrow \bar{Z}$ be the inclusions. Since $(\bar{Z}-Z) \subset \bar{Z}$ is a smooth divisor, there is by cohomological purity [1, XVI.3.9] a distinguished triangle on $\bar{Z}$

$$
\Lambda \rightarrow R j_{*} j^{*} \Lambda \rightarrow i_{*} \Lambda(-1)[-1] \rightarrow \Lambda[1]
$$


This induces a long exact sequence

$$
\cdots \rightarrow R^{s} \bar{f}_{*} \Lambda \rightarrow R^{s} f_{*} \Lambda \rightarrow R^{s-1} \bar{f}_{*}\left(i_{*} \Lambda(-1)\right) \rightarrow \cdots .
$$

Now the sheaves $R^{s} \bar{f}_{*} \Lambda$ and $R^{s-1}\left(i_{*} \Lambda(-1)\right)$ are locally constant constructible and their formation commutes with arbitrary base change [1, XVI.2.2]. In particular, the boundary map

$$
R^{0} \bar{f}_{*} i_{*} \Lambda(-1) \rightarrow R^{2} \bar{f}_{*} \Lambda
$$

is an isomorphism as this can be checked fiber by fiber. Also since $\bar{f} \circ i$ is étale we have $R^{i} \bar{f}_{*}\left(i_{*} \Lambda(-1)\right)=0$ for $i>0$. This implies that

$$
R^{0} f_{*} \Lambda \simeq R^{0} \bar{f}_{*} \Lambda, \quad R^{1} f_{*} \Lambda \simeq R^{1} \bar{f}_{*} \Lambda, \quad R^{s} f_{*} \Lambda=0 \text { for } s>1 .
$$

In particular, the sheaves $R^{s} f_{*} \Lambda$ are locally constant constructible. Since $W$ is a $K(\pi, 1)$ any cohomology class in $H^{i}\left(W, R^{j} f_{*} \Lambda\right)$ for $i>0$ is killed by a finite étale extension of $W$. We may therefore assume that $\alpha$ is given by a class in $H^{0}\left(W, R^{j} f_{*} \Lambda\right)$. Furthermore, since $R^{j} f_{*} \Lambda$ is zero for $j \geq 2$ it suffices to consider the case of $j=1$ which follows from 5.2 .

To prove 5.4 it therefore suffices by induction on $d$ to show that for any point $x \in X$ of the closed fiber there exists a neighborhood $U$ of $x$ and an elementary fibration $f: U \rightarrow W$ with $W$ smooth. For if $W^{\prime} \subset W$ is an open neighborhood of $f(x)$ which is a $K(\pi, 1)$ then 5.5 implies that $\left(U \times_{W} W^{\prime}\right)_{\bar{K}}$ is also a $K(\pi, 1)$. For this we may as well replace $V$ by its maximal unramified extension and hence may assume that the residue field $k$ is algebraically closed. Furthermore it suffices to consider the case of a $k$-rational point $x$ of $X$.

We can without loss of generality assume that $X$ is affine. Choose an immersion $X \hookrightarrow \mathbb{P}^{r}$ for some $r$, and let $\bar{X}$ be the normalization of the closure of $X$. Choose a very ample sheaf $M$ on $\bar{X}$ and let $\bar{X} \hookrightarrow \mathbb{P}_{V}^{N}$ be the embedding into projective space provided by $M^{\otimes r}$ for some $r \geq 2$. Let $Y=\bar{X}-X$ with the reduced subscheme structure, and let $Y^{o} \subset Y$ denote the open subset where $Y$ is smooth over $V$. Note that the generic fiber of $Y^{o}$ is dense in the generic fiber $Y_{K}$.

Lemma 5.6. There exist hyperplanes $H_{1}, \ldots, H_{d-1}$ in $\mathbb{P}_{V}^{N}$ containing $x$ such that the intersection $L=H_{1} \cap \cdots \cap H_{d-1}$ has relative dimension $N-d+1$ over $V$ and $L$ meets $X$ transversally. We can furthermore choose the hyperplanes $H_{1}, \ldots, H_{d-1}$ such that the intersection $L_{K} \cap \bar{X}_{K}$ is contained in the smooth locus of $\bar{X}_{K}, L_{K} \cap Y_{K}$ is contained in $Y_{K}^{o}$, and $L_{K}$ meets $\bar{X}_{K}$ and $Y_{K}^{o}$ transversally. In fact, if $\mathbb{P}$ denotes the space classifying $(d-1)$-hyperplanes in $\mathbb{P}_{V}^{N}$ containing $x$ (see the proof for a precise description of this space), then there is an open subset $\mathscr{U} \subset \mathbb{P}$ dense in every fiber such that for any morphism $\operatorname{Spec}(V) \rightarrow \mathscr{U}$ the corresponding hyperplanes $H_{1}, \ldots, H_{d-1}$ satisfy the above conditions.

Proof. Let $x: \operatorname{Spec}(V) \rightarrow X$ be a morphism sending the closed point to the previously denoted point $x$. The functor classifying hyperplanes in $\mathbb{P}^{N}$ through $x$ is easily seen to be represented by a projective smooth scheme over $V$ (and in particular with equidimensional fibers). It follows that the functor classifying $(d-1)$ hyperplanes in $\mathbb{P}^{N}$ containing $x$ is also represented by a projective smooth $V$-scheme $\mathbb{P}$.

There is an open subset $\mathscr{U}_{1} \subset \mathbb{P}$ classifying the condition that the intersection $L$ has dimension $N-d+1$ in each fiber, and the open subset $\mathscr{U}_{1}$ is dense in every fiber. The condition that $L$ meets $X$ transversally is equivalent to the condition 
that $X \cap L$ is smooth over $V$. Consider the universal intersection $\mathscr{Z}=(X \times \mathbb{P}) \cap \mathscr{L}$ over $\mathbb{P}$ where $\mathscr{L}$ denotes the intersection of the universal hyperplanes over $\mathbb{P}$. Let $\mathscr{R} \subset \mathscr{Z}$ be the closed subset where $\mathscr{Z}$ is not smooth over $\mathbb{P}$ and let $\mathscr{W} \subset \mathbb{P}$ be its image. The subset $\mathscr{W}$ is a constructible subset of $\mathbb{P}$ whose complement is dense in both the generic and closed fiber by [1, XI.2.1]. Therefore the complement $\mathscr{U}_{2} \subset \mathbb{P}$ of the closure of $\mathscr{W}$ is also dense in every fibre whence $\mathscr{U}_{1} \cap \mathscr{U}_{2}$ is also nonempty and dense in every fiber. Furthermore as in the proof of [1, XI.3.1] there exists a dense open subset $\mathscr{V} \subset \mathbb{P}_{K}$ classifying hyperplanes such that $L_{K} \cap \bar{X}_{K}$ is contained in the smooth locus of $\bar{X}_{K}$ and $L_{K} \cap Y_{K}$ is contained in $Y_{K}^{o}$. Let $Q \subset \mathbb{P}$ be the closure of the complement of $\mathscr{V}$ (with the reduced structure). The scheme $Q$ is flat over $V$ and hence the dimension of the components of the closed fiber of $Q$ is strictly smaller than the dimension of $\mathbb{P}_{k}$. In particular the intersection $Q^{c} \cap \mathscr{U}_{1} \cap \mathscr{U}_{2}$ is dense in every fiber. This implies the lemma.

By a similar reasoning we can also choose a hyperplane $H_{0}$ not containing $x$ meeting $X \cap L$ transversally and such that the intersection $Y \cap\left(H_{0} \cap L\right)_{K}=\emptyset$. If $\mathbb{P}^{\prime}$ denotes the space classifying $d$ hyperplanes $H_{0}, \ldots, H_{d-1}$, then as in the proof of 5.6 one sees that there exists an open subset $\mathscr{U}^{\prime} \subset \mathbb{P}^{\prime}$ dense in every fiber such that $H_{1}, \ldots, H_{d-1}$ satisfy the conditions of the lemma, $H_{0}$ does not contain $x, H_{0}$ meets $X \cap L$ transversally, and the intersection $Y \cap\left(H_{0} \cap L\right)_{K}$ is empty.

On $Y^{o} \subset Y$ the map $\left.\Omega_{\mathbb{P}^{N} / V}^{1}\right|_{Y^{o}} \rightarrow \Omega_{Y^{o} / V}^{1}$ is surjective. Let $\operatorname{Gr}_{\mathbb{P}^{N}}\left(\Omega_{\mathbb{P}^{N} / V}^{1}, d-1\right)$ denote the scheme over $\mathbb{P}^{N}$ classifying rank $d-1$ quotients of $\Omega_{\mathbb{P}^{N} / V}^{1}$. We then obtain a morphism $Y^{o} \rightarrow \operatorname{Gr}_{\mathbb{P}^{N}}\left(\Omega_{\mathbb{P}^{N} / V}^{1}, d-1\right)$ over $\mathbb{P}^{N}$. Let $Z \subset \operatorname{Gr}_{\mathbb{P}^{N}}\left(\Omega_{\mathbb{P}^{N} / V}^{1}, d-1\right)$ be the closure of $Y^{o}$ and let $\pi: Z \rightarrow Y$ be the projection. Then on $Z$ there is a quotient $\left.\Omega_{\mathbb{P}^{N} / V}^{1}\right|_{Z} \rightarrow \mathscr{E}$.

Lemma 5.7. As in 5.6, let $\mathbb{P}$ denote the $V$-scheme parametrizing $(d-1)$ hyperplanes in $\mathbb{P}^{N}$ through $x$, and for a (scheme-valued) point of $\mathbb{P}$ corresponding to hyperplanes $H_{1}, \ldots, H_{d-1}$ let $L$ denote the intersection $H_{1} \cap \cdots \cap H_{d-1}$. Then there exists an open subset $\mathscr{V} \subset \mathbb{P}$ dense in every fiber such that if $H_{1}, \ldots, H_{d-1}$ define a point of $\mathscr{V}$ and $z \in Z$ with image $y \in Y$ then the natural map $\Omega_{\mathbb{P}_{V}^{N} / V}^{1}(y) \rightarrow$ $\mathscr{E}(z) \oplus \Omega_{L / V}^{1}(y)$ is an isomorphism.

Similarly, if $\mathbb{P}^{\prime \prime}$ denotes the space of $(d-1)$ hyperplanes in $\mathbb{P}_{V}^{N}$ (not necessarily through $x)$ then there exists an open subset $\mathscr{V} \subset \mathbb{P}^{\prime \prime}$ dense in every fiber such that if $H_{1}, \ldots, H_{d-1}$ define a point of $\mathscr{V}$ and $z \in Z$ with image $y \in Y$ then the natural $\operatorname{map} \Omega_{\mathbb{P}_{V}^{N} / V}^{1}(y) \rightarrow \mathscr{E}(z) \oplus \Omega_{L / V}^{1}(y)$ is an isomorphism.

Proof. We give the proof of the first statement leaving to the reader the task of proving the second statement using the same argument.

Let $\mathscr{V} \subset \mathbb{P}$ be the subfunctor associating to any $V$-scheme $T$ the subset of $\mathbb{P}(T)$ classifying hyperplanes $H_{1}, \ldots, H_{d-1}$ such that the map $\left.\left.\Omega_{\mathbb{P}_{V}^{N} / V}^{1}\right|_{Z_{T}} \rightarrow \mathscr{E}\right|_{Z_{T}} \oplus$ $\left.\Omega_{L / T}^{1}\right|_{Z_{T}}$ is an isomorphism. The fact that $Z$ is proper over $V$ implies that in fact $\mathscr{V}$ is represented by an open subset of $\mathbb{P}$. We claim that $\mathscr{V}$ is dense in every fiber.

To verify this it suffices to consider the base change of $Z$ by a map $V \rightarrow \Omega$, where $\Omega$ is an algebraically closed field. We claim that for any nonempty affine open subset $U \subset Z_{\Omega}$ there exists a dense open subset $U^{\prime} \subset U$ classifying classifying hyperplanes $H_{1}, \ldots, H_{d-1}$ such that the map $\Omega_{\mathbb{P}_{V}^{N} / V}^{1}(y) \rightarrow \mathscr{E}(z) \oplus \Omega_{L / V}^{1}(y)$ is an isomorphism for every $z \in U^{\prime}$ mapping to $y \in Y_{\Omega}$. Let $X_{0}, \ldots, X_{N}$ denote the 
coordinates of $\mathbb{P}^{N}$. After shrinking on $U$ and possibly changing coordinates we can assume that $U$ maps to the open subset $\mathbb{A}_{\Omega}^{N} \subset \mathbb{P}_{\Omega}^{N}$ given by $X_{N} \neq 0$ and that $\left.\mathscr{E}\right|_{U}$ is isomorphic to $\mathscr{O}_{U}^{d-1}$. There is also a dense open subset $A \subset \mathbb{P}$ classifying hyperplanes $H_{1}, \ldots, H_{d-1}$ none of which are contained in $\left\{X_{N}=0\right\}$. A point of $A$ is given by the coefficients of linear equations

$$
\sum_{i=0}^{N-1} a_{i \nu} X_{i}=c_{\nu}
$$

where $\nu=1, \ldots, d-1$ and $c_{\nu} \in \Omega$ (in the case when we require the hyperplanes to pass through $x$ the constants $c_{\nu}$ will be fixed). Let $R$ be the coordinate ring of $U$. The basis $d X_{i}$ induces an isomorphism $\left.\Omega_{\mathbb{P}_{\Omega}^{N} / \Omega}^{1}\right|_{U} \simeq R^{N}$ and the quotient $\left.\Omega_{\mathbb{P}_{\Omega}^{N} / \Omega}^{1}\right|_{U} \rightarrow$ $\left.\Omega_{L / \Omega}^{1}\right|_{U}$ has kernel the submodule generated by the vectors $v_{\nu}=\left(a_{0 \nu}, \ldots, a_{N-1 \nu}\right)$. Let $F \subset R^{N}$ be the kernel of the surjection $\left.R^{N} \rightarrow \mathscr{E}\right|_{U}$. The intersection $I:=$ $F \cap \Omega^{N} \subset R^{N}$ must have dimension less than or equal to $N-d+1$ since the map $I \otimes_{\Omega} R \rightarrow F$ is injective (because $I \otimes_{\Omega} R \rightarrow R^{N}$ is injective) and $F$ has rank equal to $N-d+1$. From this it is clear that there exists a dense open subset of $A$ such that

$$
I \cap \operatorname{Span}\left(v_{\nu}\right)_{\nu=1}^{d-1}=\{0\} .
$$

This gives the desired dense open subset.

We can therefore choose hyperplanes $H_{0}, \ldots, H_{d-1}$ such that the conditions in 5.6 are satisfied, such that for every $z \in Z$ with image $y \in Y$ the natural map $\Omega_{\mathbb{P}_{V}^{N} / V}^{1}(y) \rightarrow \mathscr{E}(z) \oplus \Omega_{L / V}^{1}(y)$ is an isomorphism, and such that $H_{0}$ does not contain $x, H_{0}$ meets $X \cap L$ transversally and such that the intersection $Y \cap\left(H_{0} \cap L\right)_{K}$ is empty. After changing coordinates, we can further assume that $H_{0}$ is the hyperplane $\left\{X_{0}=0\right\}$. Let $C$ denote the intersection $H_{0} \cap \cdots \cap H_{d-1}$. Choosing coordinates write

$$
H_{i}: \sum_{\nu=0}^{N} a_{i \nu} X_{\nu}=0
$$

and consider the map

$$
\mathbb{P}^{N}-C \rightarrow \mathbb{P}^{d-1}, \quad y_{i}=\sum_{\nu} a_{i \nu} X_{\nu}
$$

Let $U \subset \bar{X}$ be the neighborhood of $x$ which is the complement of $H_{0} \cap \bar{X}$. We then obtain a morphism $\epsilon: U \rightarrow \mathbb{P}^{d-1}$. The fiber $\epsilon^{-1} \epsilon(x)$ is isomorphic to $U \cap L$. In fact, let $\mathbb{A}^{d-1} \subset \mathbb{P}^{d-1}$ denote the open subset where $y_{0} \neq 0$. By associating to a point $\left(y_{1}, \ldots, y_{d-1}\right)$ the hyperplanes $y_{i}-a_{i 0}=\sum_{\nu \geq 1} a_{i \nu} X_{\nu}$ we obtain a morphism $\mathbb{A}^{d-1} \rightarrow \mathbb{P}^{\prime \prime}$ over $V$. For $s \in \mathbb{A}^{d-1}$ let $L_{s}$ denote the intersection of the $(d-1)$ hyperplanes corresponding to the image of $s$. Then the fiber of $\epsilon$ over $s$ is equal to $U \cap L_{s}$. By 5.7. for general choice of $H_{0}, \ldots, H_{d-1}$, there exists an open subset $W \subset \mathbb{A}^{d-1}$ containing $\epsilon(x)$ such that for every $s \in W$ the conditions of 5.7 are satisfied for the image of $s$ in $\mathbb{P}^{\prime \prime}$. It follows that for a point $s \in W_{K}$ of the generic fiber the intersection $\bar{X} \cap L_{s}$ is smooth. Using the argument given in [1, XI, page 7 last paragraph] one shows that the map $X_{K} \rightarrow W_{K}$ is an elementary fibration.

Corollary 5.8. Let $X / V$ be a smooth proper scheme. Then for any closed point $x \in X$ the scheme $\operatorname{Spec}\left(\mathscr{O}_{X, \bar{x}}\right)_{\bar{K}}$ is a $K(\pi, 1)$. 
Proof. The scheme $\operatorname{Spec}\left(\mathscr{O}_{X, \bar{x}}\right)$ is a projective limit of $K(\pi, 1)$ 's and a standard limit argument shows that such a projective limit is also a $K(\pi, 1)$.

EXAmPLE 5.9. Let $\bar{K}$ be an algebraically closed field of characteristic 0 , and $X / \bar{K}$ a smooth $\bar{K}$-scheme with a divisor $D \subset X$ with normal crossings. Let $x \in X(\bar{K})$ be a point and let $Y$ denote $\operatorname{Spec}\left(\mathscr{O}_{X, \bar{x}}\right)$. Then if $Y^{o}$ denotes $Y \times_{X}(X-D)$ then $Y^{\circ}$ is a $K(\pi, 1)$.

For this note first that we are immediately reduced to the case when $X=$ $\mathbb{A}^{d}, x$ is the origin, and $D$ is the standard hyperplanes. By Abhyankar's lemma the fundamental group $\Delta$ of $Y^{o}$ is isomorphic to $\widehat{\mathbb{Z}}(1)^{r}$ where $r$ is the number of components of $D$ passing through $x$. Let $L$ be a locally constant sheaf of $\Lambda$-modules on $Y^{o}$. By devissage we may assume that $\Lambda=\mathbb{Z} /(p)$ and that $L$ corresponds to a simple representation of $\Delta$. In this case $L$ is isomorphic to a tensor product $L_{\mu_{1}} \otimes \cdots \otimes L_{\mu_{r}}$ where $L_{\mu_{j}}$ is a representation of the $j$-th factor of $\widehat{\mathbb{Z}}(1)$ in $\Delta$. By the Künneth formula this further reduces the proof to the case when $X=\mathbb{A}^{1}$ and $D$ is the origin. In this case $Y^{o}$ is a projective limit of $K(\pi, 1)$ 's and hence is also a $K(\pi, 1)$.

This argument also proves the following. Assume given an étale morphism

$$
\pi: X \rightarrow \operatorname{Spec}\left(\bar{K}\left[X_{1}, \ldots, X_{d}\right]\right)
$$

such that $D$ is equal to the inverse image of the standard hyperplanes. Let $j$ : $X^{o}:=(X-D) \hookrightarrow X$ be the inclusion and let $j$ be an integer. For $\underline{n}=\left(n_{1}, \ldots, n_{d}\right)$ let $X_{n} \rightarrow X$ denote the scheme obtained by taking the $n_{i}$-th roots of $X_{i}$, and let $j_{n}: X_{n}^{o} \hookrightarrow X_{n}$ be the inclusion. Then for any locally constant constructible sheaf $L$ and cohomology class $\alpha \in R^{i} j_{*} L(i>0)$ there exists integers $\underline{n}$ such that the image of $\alpha$ in $R^{i} j_{n *} L$ is zero, $j_{n *} L$ is locally constant constructible, and the natural map $j_{n}^{*} j_{n *} L \rightarrow L$ is an isomorphism. Indeed this can be verified after passing to the strict henselization of $X$ at a point in which case it follows from the above computations.

Corollary 5.10. Let $X$ be a smooth proper $V$-scheme and $D \subset X$ a divisor with normal crossings relative to $V$. Let $X^{o}$ denote the complement of $D$. Then for any closed point $x \in X$ the scheme $\left(\operatorname{Spec}\left(\mathscr{O}_{X, \bar{x}}\right)_{\bar{K}}\right)^{o}:=X_{\bar{K}}^{o} \times_{X} \operatorname{Spec}\left(\mathscr{O}_{X, \bar{x}}\right)$ is a $K(\pi, 1)$.

Proof. Let $X^{\dagger}$ denote $\operatorname{Spec}\left(\mathscr{O}_{X, \bar{x}}\right)$ and let $L$ be a locally constant constructible sheaf on $X_{\bar{K}}^{\dagger o}$. We have to show that for any cohomology class $\alpha \in H^{i}\left(X_{\frac{\dagger o}{K}}^{\dagger}, L\right)$ with $i>0$ there exists a finite étale surjection $V \rightarrow X_{\bar{K}}^{\dagger o}$ over which $\alpha$ becomes zero. Choose a formally étale morphism

$$
\pi: X^{\dagger} \rightarrow \operatorname{Spec}\left(V\left[X_{1}, \ldots, X_{d}\right]\right)
$$

such that $D$ is equal to the inverse image of $\left\{X_{1} \cdots X_{d}=0\right\}$. Let $j: X^{\dagger o} \hookrightarrow X^{\dagger}$ be the inclusion. By 5.9 and consideration of the Leray spectral sequence

$$
E_{2}^{p q}=H^{p}\left(X_{\bar{K}}^{\dagger}, R^{q} j_{*} L\right) \Longrightarrow H^{p+q}\left(X_{\frac{\dagger o}{K}}^{\dagger o}, L\right)
$$

there exists integers $\underline{n}=\left(n_{1}, \ldots, n_{d}\right)$ such that if $X_{n}^{\dagger}$ denotes the scheme

$$
X \times_{\operatorname{Spec}\left(V\left[X_{1}, \ldots, X_{d}\right]\right)} \operatorname{Spec}\left(V\left[X_{1}^{1 / n_{1}}, \ldots, X_{d}^{1 / n_{d}}\right]\right)
$$

with inclusion $j_{n}: X_{n}^{\dagger o} \hookrightarrow X_{n}^{\dagger}$ then $\alpha$ is given by a class in $H^{i}\left(X_{n, \bar{K}}^{\dagger}, j_{n *} L\right), j_{n *} L$ is locally constant constructible, and $j_{n}^{*} j_{n *} L \rightarrow L$ is an isomorphism. Replacing $X^{\dagger}$ 
by $X_{n}^{\dagger}$ we may therefore assume that $L$ extends to a sheaf on $X_{\bar{K}}^{\dagger}$ which we again denote by $L$ and that $\alpha$ is obtained from a cohomology class in $H^{i}\left(X_{\bar{K}}^{\dagger}, L\right)$. Since $X_{\bar{K}}^{\dagger}$ is a $K(\pi, 1)$ the result follows.

In fact 5.4 can be generalized as follows to also take into account a boundary.

TheOREM 5.11. With notation as in 5.4, let $D \subset X$ be a divisor with simple normal crossings relative to $V$, set $X^{o}:=X-D$. Let $D=D_{1} \cup \cdots \cup D_{n}$ with each $D_{i}$ an irreducible divisor. For a subset $A \subset\{1, \ldots, n\}$ (possibly empty) let $D_{A}$ denote the intersection $\cap_{i \in A} D_{i}$, and let $D_{A}^{o}$ denote $D_{A}-\left(\cup_{j \notin A} D_{j}\right)$. Then for any point $x \in X$ in the closed fiber there exists a Zariski open neighborhood $U$ of $x$ such that each $D_{A, \bar{K}}^{o} \cap U_{\bar{K}}$ is a $K(\pi, 1)$.

Proof. Note first that after possibly replacing the polarization $M$ giving the embedding $X \hookrightarrow \mathbb{P}^{N}$ by $M^{\otimes r}$ for some $r$, we may assume that the closure $\bar{D}_{i} \subset \bar{X}$ of each irreducible component $D_{i}$ is normal. Let $\epsilon: X \rightarrow W$ be the elementary fibration constructed in the proof of 5.4 by projection from the hyperplanes $H_{i}$. Then an examination of the proof and standard Bertini type arguments shows that we can furthermore choose the hyperplanes $H_{1}, \ldots, H_{d-1}$ so that if $L=H_{1} \cap \cdots \cap$ $H_{d-1}$ then the intersection $L_{\bar{K}} \cap \bar{D}_{i, \bar{K}}$ is transverse for all $i$ so that this intersection is an étale $\bar{K}$-scheme, and furthermore can choose the $H_{i}$ 's such that the projection of the double intersections $D_{i} \cap D_{j}$ have image a divisor with simple normal crossings in $W$. Furthermore, we can choose the hyperplane $H_{0}$ so that it does not meet the intersections $L_{\bar{K}} \cap \bar{D}_{i, \bar{K}}$. Then it follows from the proof that after some shrinking on $W$ the schemes $\bar{D}_{i, \bar{K}}$ are finite and étale over $W_{\bar{K}}$. This in turn implies that the map $X_{\bar{K}}^{o} \rightarrow W_{\bar{K}}$ is also an elementary fibration whence $X_{\bar{K}}^{o}$ is a $K(\pi, 1)$, and the statement about the higher intersections follows by induction.

\section{The topos $\mathscr{X}_{\bar{K}}^{o}$}

6.1. Let $X / V$ be a smooth $V$-scheme, and let $D \subset X$ be a divisor with normal crossings relative to $V$. For an $X$-scheme $\pi: U \rightarrow X$ let $U^{o}$ denote $U-\pi^{-1}(D)$.

Define a site $\mathscr{S}$ as follows. The objects of $\mathscr{S}$ are pairs $(U, N)$, where $U$ is an étale separated $X$-scheme of finite type and $N \rightarrow U_{\bar{K}}^{o}$ is a finite and étale morphism. A morphism $\left(U^{\prime}, N^{\prime}\right) \rightarrow(U, N)$ is given by a commutative diagram of $X$-morphisms

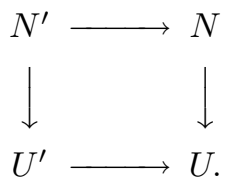

A collection of morphisms $\left\{\left(U_{i}, N_{i}\right) \rightarrow(U, N)\right\}$ is defined to be a covering if $\left\{U_{i} \rightarrow\right.$ $U\}$ is a covering in $\operatorname{Et}(X)$ and $\left\{N_{i} \rightarrow N\right\}$ is a covering in $\operatorname{Et}\left(X_{\bar{K}}^{o}\right)$. We write $\mathscr{X}_{\bar{K}}^{o}$ for the resulting topos.

Lemma 6.2. The category $\mathscr{S}$ has finite projective limits.

Proof. By [1, I.2.3], it suffices to show that finite products and equalizers are representable in $\mathscr{S}$. If $\left\{\left(U_{i}, N_{i}\right)\right\}_{i=1}^{n}$ is a finite set of objects in $\mathscr{S}$, then their 
product is given by the pair

$$
\left(U_{1} \times{ }_{X} U_{2} \times \cdots \times{ }_{X} U_{n}, N_{1} \times X_{K}^{o} N_{2} \times \cdots \times \times_{X_{K}^{o}} N_{n}\right) .
$$

For the existence of equalizers, consider two morphisms $f, g:\left(U_{1}, N_{1}\right) \rightarrow\left(U_{2}, N_{2}\right)$ in $\mathscr{S}$, and let $N^{\dagger}$ (resp. $U^{\dagger}$ ) denote the equalizer of the morphisms $N_{1} \rightrightarrows N_{2}$ (resp. $\left.U_{1} \rightrightarrows U_{2}\right)$. Then $\left(U^{\dagger}, N^{\dagger}\right)$ represents the equalizer of $f$ and $g$. The only point that needs verification is that $N^{\dagger}$ is finite over $U_{\bar{K}}^{\dagger o}$. For this note that $N^{\dagger}$ is equal to the fiber product of the diagram

$$
N_{1} \stackrel{a \times b}{\longrightarrow} N_{2}{ }_{X_{\bar{K}}^{o}}^{N_{2}} N_{2},
$$

where we write $a, b: N_{1} \rightarrow N_{2}$ for the two morphisms. Since $N_{2} \rightarrow X_{\bar{K}}^{o}$ is separated this implies that $N^{\dagger}$ is a closed subscheme of $N_{1}$. By a similar argument $U^{\dagger}$ is a closed subscheme of $U_{1}$. We therefore have a commutative diagram

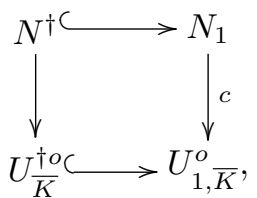

where the horizontal arrows are closed immersions, and $c$ is finite. It follows that $N^{\dagger} \rightarrow U_{\bar{K}}^{\dagger o}$ is also finite.

REMARK 6.3. A similar argument to the one in the proof of 6.2 shows that a finite projective limit of finite étale morphisms is finite and étale. Indeed to verify this it suffices to consider finite products (immediate) and equalizers (proof of 6.2).

6.4. There is a continuous morphism of sites $\mathscr{S} \rightarrow \operatorname{Et}\left(X_{\bar{K}}^{o}\right)$ sending $(U, N)$ to the composite $N \rightarrow U_{\bar{K}}^{o} \rightarrow X_{\bar{K}}^{o}$. This functor preserves finite inverse limits since it preserves products and equalizers, and therefore by [1, IV.4.9.2] induces a morphism of topoi

$$
u_{X}: X_{\bar{K}, \text { et }}^{o} \rightarrow \mathscr{X}_{\bar{K}}^{o}
$$

There is also a continuous morphism of sites $\operatorname{Et}(X) \rightarrow \mathscr{S}$ sending $U \rightarrow X$ to $\left(U\right.$, id $: U_{\bar{K}}^{o} \rightarrow U_{\bar{K}}^{o}$ ). This functor also preserves finite projective limits, and induces a morphism of topoi

$$
\nu_{X}: \mathscr{X}_{\bar{K}}^{o} \rightarrow X_{\text {et }} .
$$

Note that the composite $\nu_{X} \circ u_{X}: X_{\bar{K}}^{o}$,et $\rightarrow X_{\text {et }}$ is just the morphism of topoi induced by the morphism of schemes $X_{\bar{K}}^{o} \rightarrow X$.

Finally there is a morphism of $\operatorname{sites} \operatorname{Fet}\left(X_{\bar{K}}^{o}\right) \rightarrow \mathscr{S}$ sending $N \rightarrow X_{\bar{K}}^{o}$ to the object $(X, N) \in \mathscr{S}$. This morphism induces a morphism of topoi

$$
\epsilon_{X}: \mathscr{X}_{\bar{K}}^{o} \rightarrow X_{\bar{K}, \mathrm{Fet}}^{o} \cdot
$$

Observe that $\epsilon_{X} \circ u_{X}$ is the natural projection $X_{\bar{K}, \text { et }}^{o} \rightarrow X_{\bar{K}, \mathrm{Fet}}^{o}$.

Lemma 6.5. Let $L$ be a locally constant constructible abelian sheaf on $X_{\bar{K}}^{o}$. Then $R^{i} u_{X *} L=0$ for $i>0$ and $u_{X *} L$ is the sheaf $(U, N) \mapsto L(N)$. 
Proof. Fix an integer $i>0$. By 5.11 any object of $\mathscr{S}$ can be covered by objects $(U, N)$ with $U^{o}$ a $K(\pi, 1)$. To prove that $R^{i} u_{X *} L=0$, it therefore suffices to show that for any such $(U, N) \in \mathscr{S}$ and class $o \in H^{i}\left(\left.X_{\bar{K}, \mathrm{et}}^{o}\right|_{u_{X}^{-1}(U, N)}, L\right)$, there exists a covering $\left(U^{\prime}, N^{\prime}\right) \rightarrow(U, N)$ such that the pullback of $o$ to $H^{i}\left(\left.X_{\bar{K}, \mathrm{et}}^{o}\right|_{u_{X}^{-1}\left(U^{\prime}, N^{\prime}\right)}, L\right)$ is zero.

For this note that for any $(U, N) \in \mathscr{S}$ there is a natural isomorphism

$$
H^{i}\left(\left.X_{\bar{K}, \text { et }}^{o}\right|_{u_{X}^{-1}(U, N)}, L\right) \simeq H^{i}\left(N_{\text {et }}, L\right) .
$$

Now if $U^{o}$ is a $K(\pi, 1)$, then $N$ is also a $K(\pi, 1)$. This implies that there exists a finite étale covering $N^{\prime} \rightarrow N$ such that $o$ maps to zero in $H^{i}\left(N_{\text {et }}^{\prime}, L\right)$. The resulting covering $\left(U, N^{\prime}\right) \rightarrow(U, N)$ in $\mathscr{S}$ then kills $o$.

The description of $u_{X *} L$ is immediate from the definitions.

Lemma 6.6. Assume that $X^{o}$ is a $K(\pi, 1)$. Then for any abelian sheaf $F$ in $X_{\bar{K}, \text { Fet }}^{o}$, the natural map $F \rightarrow R \epsilon_{X *} \epsilon_{X}^{*} F$ is an isomorphism.

Proof. Note that by the preceding lemma, $R \epsilon_{X *} \epsilon_{X}^{*} F \simeq R\left(\epsilon_{X} \circ u_{X}\right)_{*}\left(\left.F\right|_{X_{\bar{K}, \text { et }}^{o}}\right)$. The lemma therefore follows from the fact that $X^{o}$ is a $K(\pi, 1)$ which implies that the adjunction map $F \rightarrow R\left(\epsilon_{X} \circ u_{X}\right)_{*}\left(\left.F\right|_{X_{K, \text { et }}^{o}}\right)$ is an isomorphism.

6.7. An annoying technical difficulty about the topos $\mathscr{X}_{\bar{K}}^{o}$ is that most of the sheaves we will consider will be obtained from presheaves by sheafification. This means that if $U \in \operatorname{Et}(X)$ then it is rather difficult to describe the restriction of the sheaf to $U_{\bar{K}, \text { Fet }}^{o}$.

We can overcome this as follows. Recall from [1, V, §7] (in particular [1, V.7.4.1 (4)]) the following way of computing cohomology using hypercovers. Let $S$ be a site with representable finite products and fiber products and let $S^{\sim}$ be the associated topos. Let $H R_{S}$ (or just $H R$ if the reference to the site $S$ is clear) denote the homotopy category of hypercovers in $S$. This is the category whose objects are hypercovers by objects of $S$ of the initial object of the topos $S^{\sim}$ and whose morphisms are homotopy classes of morphisms of hypercovers (see [1, V.7.3.1.6] for the notion of a homotopy between two morphisms of hypercovers). By [1, V.7.3.2 (1)] the opposite category $H R^{o}$ is filtering (while the category of hypercovers before passing to the homotopy category is not filtering). Let $G$ be a presheaf of abelian groups on $S$ with associated sheaf $G^{a}$. For any hypercover $U$. of the initial object in $S^{\sim}$, let $G\left(U\right.$.) denote the complex obtained by evaluating $G$ on each of the $U_{n}$ to get a cosimplicial abelian group and then taking the total complex. Two homotopic morphisms $f, g: U^{\prime} \rightarrow U$. induce the same map $H^{*}\left(G\left(U^{\prime}\right)\right) \rightarrow H^{*}\left(G\left(U^{\prime}\right)\right)$ and hence one can form the limit

$$
\lim _{U \cdot \overrightarrow{\in H} R} H^{*}(G(U .))
$$

There is a natural morphism

$$
\lim _{U . \overrightarrow{\in H} R} H^{*}(G(U .)) \rightarrow H^{*}\left(S, G^{a}\right)
$$

which by [1, 7.4.1 (4)] is an isomorphism, where $\mathrm{sq}_{k}$ (resp. $\operatorname{cosq}_{k}$ ) denotes the $k$-th skeleton (resp. coskeleton) functor. For a fixed $q$, one can do slightly better. Namely, recall that for an integer $k$ an object $U . \in H R$ is $k$-truncated if the 
adjunction map

$$
U . \rightarrow \operatorname{cosq}_{k} \mathrm{sq}_{k} U \text {. }
$$

is an isomorphism. We denote by $H R_{k} \subset H R$ the full subcategory of $k$-truncated objects. By [1, V.7.3.2 (1)] the category $H R_{k}^{o}$ is also filtering, and by [1, 7.4.1 (3)] the natural map

$$
\lim _{U . \overrightarrow{\in H} R_{k}} H^{q}(G(U .)) \rightarrow H^{q}(S, F)
$$

is an isomorphism for any $k>q$.

6.8. Now let $\mathscr{S}$ be as in 6.1. Let $F$ be a presheaf on $\mathscr{S}$ such that for every $U \in \operatorname{Et}(X)$ the restriction $F_{U}$ of $F$ to $\operatorname{Fet}\left(U_{\bar{K}}^{o}\right)$ is a sheaf. Let $\nu_{X}: \mathscr{X}_{\bar{K}}^{o} \rightarrow X_{\text {et }}$ be the morphism of topoi defined in 6.4. For any $U \in \operatorname{Et}(X)$ let $\left.\mathscr{S}\right|_{U}$ denote the site of objects in $\mathscr{S}$ with a morphism to the object $\left(U, U_{\bar{K}}^{o}\right)$. The topos associated to $\left.\mathscr{S}\right|_{U}$ is the localized topos $\left.\mathscr{X}_{\frac{o}{K}}^{o}\right|_{U}$. We also have the morphism of topoi

$$
\epsilon_{U}:\left.\mathscr{X}_{\bar{K}}^{o}\right|_{U} \rightarrow U_{\bar{K}, \mathrm{Fet}}^{o} .
$$

The map of presheaves $F \rightarrow F^{a}$ induces a morphism $F_{U} \rightarrow R \epsilon_{U *} F^{a}$. Applying the functor $H^{q}\left(U_{\bar{K}, \text { Fet }}^{o},-\right)$ we obtain a morphism

$$
H^{q}\left(U_{\bar{K}, \mathrm{Fet}}^{o}, F_{U}\right) \rightarrow H^{q}\left(\left.\mathscr{X}_{\bar{K}}^{o}\right|_{U}, F^{a}\right) .
$$

This map is functorial in $U$, so if $\mathscr{H}^{q}(F)$ denotes the sheaf on $\operatorname{Et}(X)$ associated to the presheaf

$$
U \mapsto H^{q}\left(U_{\bar{K}, \mathrm{Fet}}^{o}, F_{U}\right)
$$

we obtain a morphism of sheaves on $\operatorname{Et}(X)$

$$
\mathscr{H}^{q}(F) \rightarrow R^{q} \nu_{X *} F^{a} .
$$

Proposition 6.9. The morphism 6.8.4 is an isomorphism.

Proof. The key point is the following lemma:

Lemma 6.10. Let $U \in \operatorname{Et}(X)$ and let $\left(U_{.}^{\prime}, N_{.}^{\prime}\right) \rightarrow\left(U, U_{\bar{K}}^{o}\right)$ be a $k$-truncated hypercover in $\mathscr{S}$. Then after replacing $U$ by an étale cover $W \rightarrow U$ and $\left(U^{\prime}, N^{\prime}\right) \rightarrow$ $\left(U, U_{\bar{K}}^{o}\right)$ by $\left(U^{\prime} \times_{U} W, N^{\prime} \times_{U} W\right) \rightarrow\left(W, W_{\bar{K}}^{o}\right)$ there exists a morphism of $k$-truncated hypercovers $\left(U^{\prime \prime}, N_{.}^{\prime \prime}\right) \rightarrow\left(U^{\prime}, N_{.}^{\prime}\right)$ over $\left(U, U_{\bar{K}}^{o}\right)$ such that each $N_{n}^{\prime \prime}$ is finite and étale over $U_{\bar{K}}^{o}$.

Proof. By a standard limit argument, it suffices to consider the case when $U$ is the spectrum of a strictly henselian local ring (indeed the assumption that the hypercovers are $k$-truncated ensures that they are given by finitely many schemes and maps, and hence if we prove the lemma over the strict henselization of a geometric point $\bar{x} \rightarrow U$ we can "spread out" to get the lemma in some étale neighborhood of $\bar{x})$.

In this case any étale $U$-scheme $Z \rightarrow U$ decomposes canonically as $Z^{g} \amalg Z^{f}$, where $Z^{f} \rightarrow U$ is finite étale and the inclusion $Z^{f} \hookrightarrow Z$ reduces to an isomorphism over the closed point of $U$. This decomposition is functorial in $Z$. Let $U^{\prime f}$ be the simplicial $U$-scheme $[n] \mapsto U_{n}^{\prime f}$. The simplicial scheme $U^{\prime f}$ is again a hypercover 
of $U$. Indeed for any integer $n \geq 0$ to verify that the morphism of finite étale $U$-schemes

$$
U_{n+1}^{\prime f} \rightarrow\left(\operatorname{cosq}_{n} \mathrm{sq}_{n} U^{\prime f}\right)_{n+1}
$$

is surjective, it suffices to check that it induces a surjection over the closed point of $U$ which follows from the fact that $U^{\prime} \rightarrow U$ is a hypercover.

We claim that in this local case we can find a morphism $\left(U_{.}^{\prime \prime}, N_{.}^{\prime \prime}\right) \rightarrow\left(U_{.}^{\prime}, N^{\prime}\right)$ as in the lemma with $U_{!^{\prime \prime}}=U^{\prime f}$. We prove this stronger statement by induction on $k$.

For the case $k=0$, we have a commutative diagram

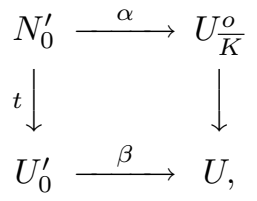

where $\alpha$ and $\beta$ are étale and surjective, and $\left(U^{\prime}, N^{\prime}\right) \rightarrow\left(U, U_{\bar{K}}^{o}\right)$ is obtained by taking the 0 -coskeletons of the horizontal arrows. Set $N_{0}^{\prime f}:=N_{0}^{\prime} \times_{U_{0}^{\prime}} U_{0}^{\prime f}$. We then obtain a commutative diagram mapping to 6.10 .2

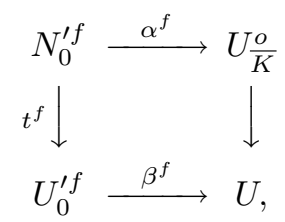

where the map $N_{0}^{\prime f} \rightarrow U_{0, \bar{K}}^{\prime f o}$ induced by $t^{f}$ is finite and étale, and $\alpha^{f}$ and $\beta^{f}$ are étale surjections. Taking 0 -coskeletons of the horizontal arrows in 6.10 .3 we obtain the desired hypercover in $\mathscr{S}$ mapping to $\left(U_{.}^{\prime}, N_{.}^{\prime}\right) \rightarrow\left(U, U_{\bar{K}}^{o}\right)$.

For the inductive step we assume the lemma holds for $k$ and prove it for $k+1$. By induction we can find a hypercover

$$
\left(\operatorname{cosq}_{k} \operatorname{sq}_{k} U^{\prime f}, Z .\right) \rightarrow\left(U, U \frac{o}{K}\right)
$$

and a commutative diagram over $U$

Set

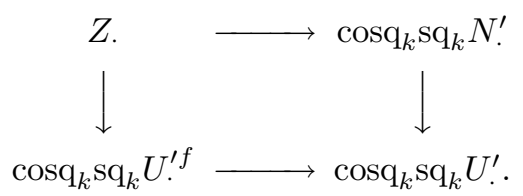

$$
W:=N_{k+1} \times\left(\operatorname{cosq}_{k} \mathrm{sq}_{k} N .\right)_{k+1}\left(\operatorname{cosq}_{k} \mathrm{sq}_{k} Z .\right)_{k+1}
$$

so that there is a commutative diagram (whose top square is cartesian)

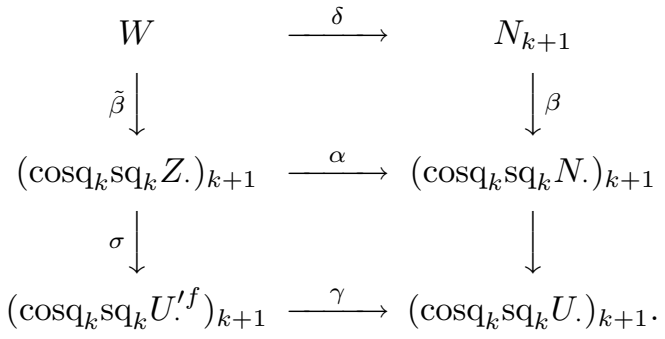


The morphism

$$
\sigma^{\prime}:\left(\operatorname{cosq}_{k} \mathrm{sq}_{k} Z .\right)_{k+1} \rightarrow\left(\operatorname{cosq}_{k} \mathrm{sq}_{k} U_{,, \bar{K}}^{\prime f o}\right)_{k+1}
$$

induced by $\sigma$ is finite and étale being an inverse limit of finite étale morphisms 6.3. It follows that $\left(\operatorname{cosq}_{k} \mathrm{sq}_{k} Z \text {. }\right)_{k+1}$ is proper over $U_{\bar{K}}^{o}$, and therefore the morphism $\alpha$ is also finite and étale. Since the top square is cartesian we conclude that $\delta$ is also finite étale. The morphism $\tilde{\beta}$ is surjective since $\beta$ is surjective (since $N$. is a hypercover).

Next set

$$
\widetilde{W}:=U_{k+1}^{\prime f} \times\left(\operatorname{cosq}_{k} \mathrm{sq}_{k} U^{\prime f}\right)_{k+1} W .
$$

Since $U^{\prime f}$ is a hypercover of $U$, the morphism $U_{k+1}^{\prime f} \rightarrow\left(\operatorname{cosq}_{k} \operatorname{sq}_{k} U^{\prime f}\right)_{k+1}$ is surjective and finite. This implies that the map $\widetilde{W} \rightarrow W$ is also surjective and that $\widetilde{W}$ is finite over $U_{\bar{K}}^{o}$. Define

Let $\underline{\Delta}_{k+1} \subset \underline{\Delta}$ denote the full subcategory whose objects are $\{[s] \mid s \leq k+1\}$.

$$
R: \underline{\Delta}_{k+1}^{o} \rightarrow\left(U_{\bar{K}}^{o} \text { schemes }\right)
$$

as follows. For $s<k+1$ set $R_{s}:=Z_{s}$, and set $R_{k+1}:=\widetilde{W}$. For a morphism $\delta:[s] \rightarrow[k+1]$ with $s<k+1$ define $\delta^{*}: R_{k+1} \rightarrow R_{s}$ to be the composite

$$
\widetilde{W} \longrightarrow\left(\operatorname{cosq}_{k} \mathrm{sq}_{k} Z\right)_{k+1} \longrightarrow Z_{s},
$$

where the second morphism is the one given by the simplicial structure on $\operatorname{cosq}_{k} \mathrm{sq}_{k} Z$.. For a morphism $\delta:[k+1] \rightarrow[s]$ let $\delta^{*}: R_{s} \rightarrow R_{k+1}=\widetilde{W}$ be the morphism defined as follows. First the commutative diagram

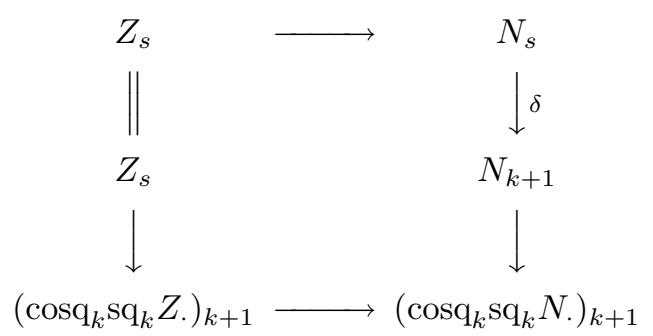

defines a morphism $h: Z_{s} \rightarrow W$. This morphisms sits in a commutative diagram

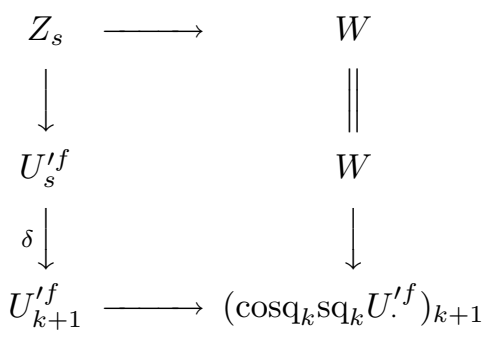

and hence factors through a morphism $\delta^{*}: R_{s}=Z_{s} \rightarrow \widetilde{W}=R_{k+1}$. We leave to the reader the verification that these definitions are compatible with compositions of morphisms in $\underline{\Delta}_{k+1}$. Since all the morphisms in the diagram

$$
\widetilde{W} \longrightarrow W \longrightarrow\left(\operatorname{cosq}_{k} \mathrm{sq}_{k} Z \cdot\right)_{k+1}
$$


are surjections the truncated simplicial scheme $R$ is a hypercover of $U_{\bar{K}}^{o}$. Furthermore we have a commutative diagram of truncated simplicial schemes

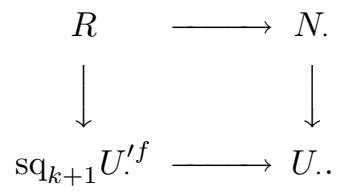

Taking $(k+1)$-coskeletons we obtain the desired morphism of hypercovers in $\mathscr{S}$.

To deduce 6.9 from this, let $s \in R^{q} \epsilon_{X *} F^{a}$ be a section over some $U \in \operatorname{Et}(X)$. After shrinking on $U$ we can find a hypercover $\left(U^{\prime}, N^{\prime}\right) \rightarrow\left(U, U_{\bar{K}}^{o}\right)$ and a section $\tilde{s} \in F_{U_{q}^{\prime}}\left(N_{q}^{\prime}\right)$ representing the class $s$. By 6.10, after further shrinking on $U$, we can assume that each $U_{n}^{\prime}$ is finite étale over $U$. In this case $N$. is a hypercover of $U_{\bar{K}}^{o}$

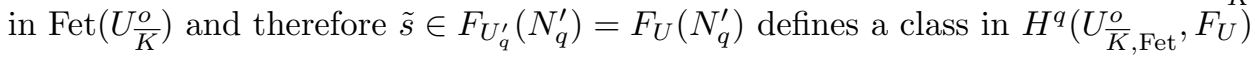
mapping to $s$. This shows that 6.8.4 is surjective.

For the injectivity of 6.8.4 consider $U \in \operatorname{Et}(X)$ and an element

$$
\sigma \in H^{q}\left(U_{\bar{K}, \mathrm{Fet}}^{o}, F_{U}\right)
$$

mapping to zero in $R^{q} \nu_{X *} F^{a}$. After shrinking on $U$, we can find an étale hypercover $N$. $\rightarrow U_{\bar{K}}^{o}$ in $\operatorname{Fet}\left(U_{\bar{K}}^{o}\right)$, a section $\tilde{\sigma} \in F_{U}\left(N_{q}\right)$ defining $\sigma$, and a morphism of hypercovers in $\mathscr{S}$

$$
\left(U_{.}^{\prime}, N^{\prime}\right) \rightarrow(U, N .)
$$

such that $\tilde{\sigma}$ maps to a boundary in $F_{U^{\prime}}\left(N^{\prime}\right)$. By 6.10 after shrinking on $U$ we can assume each $U_{n}^{\prime}$ is finite and étale over $U$. In this case $N^{\prime} \rightarrow N$. is a morphism of hypercovers in $U_{\bar{K}}^{o}$, Fet such that the image of $\tilde{\sigma}$ under the map $F_{U}(N$. $) \rightarrow F_{U}\left(N^{\prime}\right)$ is a boundary. Therefore $\tilde{\sigma}$ defines the zero section of $\mathscr{H}^{q}$. This completes the proof of 6.9 .

Corollary 6.11. With notation and assumptions as in 6.8, the natural map (6.11.1)

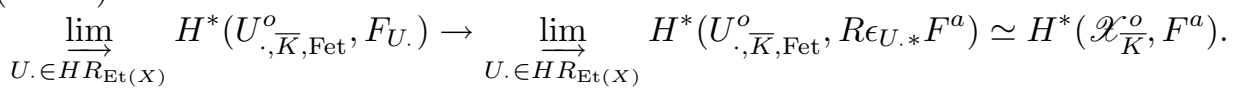

is an isomorphism.

Proof. For any $U . \in H R_{\mathrm{Et}(X)}$ there is a canonical spectral sequence

$$
E_{1}^{p q}=H^{q}\left(U_{p, \bar{K}, \mathrm{Fet}}^{o}, F_{U_{p}}\right) \Longrightarrow H^{p+q}\left(U_{\cdot, \bar{K}, \mathrm{Fet}}^{o}, F_{U}\right)
$$

and similarly

$$
E_{1}^{p q}=H^{q}\left(U_{p, \bar{K}, \mathrm{Fet}}^{o}, R \epsilon_{U_{p} *} F^{a}\right) \Longrightarrow H^{p+q}\left(U_{\cdot, \bar{K}, \mathrm{Fet}}^{o}, R \epsilon_{U . *} F^{a}\right),
$$

and it follows from the construction that the morphism 6.11.1 is induced by a morphism of spectral sequences from 6.11.2 to 6.11.3. The $E_{2}^{p q}$-term of 6.11 .2 computes the $p$-th Cech cohomology of the presheaf

$$
U \mapsto H^{q}\left(U_{\bar{K}, \text { Fet }}^{o}, F_{U}\right)
$$

and the $E_{2}^{p q}$-term of 6.11 .3 computes the $p$-th Cech cohomology of the presheaf

$$
U \mapsto H^{q}\left(\left.\mathscr{X}_{\bar{K}}^{o}\right|_{U}, F^{a}\right) .
$$

Proposition 6.9 therefore shows that the map from 6.11.2 to 6.11 .3 induces an isomorphism on $E_{2}$-terms when passing to the limit over all $U$. $\in H R_{\operatorname{Et}(X)}$. 
6.12. Let $\mathscr{O}$ denote the sheaf on $\mathscr{S}$ which to any $(U, N)$ associates $\Gamma\left(U, \mathscr{O}_{U}\right)$. Define $\mathscr{O}_{\mathscr{X}_{\frac{o}{K}}}$ to be the sheaf associated to the presheaf $\widetilde{\mathscr{O}}_{\mathscr{X}_{\frac{o}{K}}}$ sending $(U, N)$ to the global sections of the normalization of $U$ in $N$. Also define $\mathscr{J}_{X} \subset \mathscr{O}_{\mathscr{X}_{\frac{o}{K}}}$ to be the sheaf of ideals associated to the presheaf $\widetilde{\mathscr{J}}_{X}$ which to any $(U, N)$ associates the ideal of the inverse image (with the reduced structure) of $D$ in the normalization $\bar{N}$ of $U_{\bar{K}}$ in $N$.

If $U=\operatorname{Spec}(R)$ is an affine étale $X$-scheme admitting a morphism as in 2.16.1 and if $\Delta_{U}$ denotes the fundamental group of $U_{\bar{K}}^{o}$ with respect to a generic base point, then the sheaf $\widetilde{\mathscr{O}}_{\mathscr{X}_{\bar{K}}, U}$ (resp. $\widetilde{\mathscr{J}}_{X, U}$ ) is the sheaf on $U_{\bar{K} \text {, Fet }}^{o}$ corresponding to the $\Delta_{U}$-representation $\bar{R}$ (resp. $\bar{J}_{X}$ ) defined in 3.7 . From this, 4.7 , and 3.17 we conclude:

Corollary 6.13. For any $r \geq 1$, the sheaves

$$
R^{i} \nu_{X *} \mathscr{O}_{\mathscr{X}_{\frac{o}{K}}} / p^{r} \mathscr{O}_{\mathscr{X}_{\frac{o}{K}}}, \quad \text { and } R^{i} \nu_{X *} \mathscr{J}_{X} / p^{r} \mathscr{J}_{X}
$$

are almost isomorphic to quasi-coherent sheaves on $X_{\mathrm{et}}$, are almost zero for $i>d$, and there is a canonical trace morphism

$$
\operatorname{tr}: R^{d} \nu_{X *} \mathscr{J}_{X} / p^{r} \mathscr{J}_{X} \rightarrow \Omega_{X / V}^{d} \otimes_{V}\left(\bar{V} / p^{r}\right)(-d)
$$

defined in $\mathscr{O}_{X}^{a}-$ Mod.

Corollary 6.14. Assume that $X$ is smooth and proper over $\operatorname{Spec}(V)$. Then $H^{i}\left(\mathscr{X}_{\bar{K}}^{o}, \mathscr{J}_{X} / p^{r} \mathscr{J}_{X}\right)$ is almost zero for $i>2 d$ and there is a canonical trace morphism defined in $\bar{V}^{a}-$ Mod

$$
\operatorname{tr}: H^{2 d}\left(\mathscr{X}_{\bar{K}}^{o}, \mathscr{J}_{X} / p^{r} \mathscr{J}_{X}\right) \rightarrow \bar{V} / p^{r} \bar{V}(-d)
$$

PROOF. By the preceding corollary and standard facts about cohomology of quasi-coherent sheaves, we obtain that $H^{i}\left(\mathscr{X}_{\bar{K}}^{o}, \mathscr{J}_{X} / p^{r} \mathscr{J}_{X}\right)$ is almost zero for $i>2 d$ and that for $i=2 d$ this group maps to $H^{d}\left(X, \Omega^{d} \otimes \bar{V} / p^{r} \bar{V}\right)(-d)$. By Serre duality there is a canonical isomorphism

$$
H^{d}\left(X, \Omega^{d} \otimes \bar{V} / p^{r} \bar{V}\right)(-d) \simeq H^{0}\left(X \otimes\left(V / p^{r}\right), \mathscr{O}_{X \otimes V / p^{r}}\right) \otimes \bar{V}(-d)
$$

which implies the result.

By the same argument one obtains the following:

Corollary 6.15. Assume that $X$ is smooth and proper over $\operatorname{Spec}(V)$. Let $L$ be a locally constant sheaf of free $\mathbb{Z} / p^{r} \mathbb{Z}-$ modules on $X_{\bar{K}}^{o}$, let $L^{*}:=\mathscr{H}$ om $\left(L, \mathbb{Z} / p^{r}\right)$ denote its dual, and set $\mathscr{L}:=u_{X *} L$ and $\mathscr{L}^{*}:=u_{X *} L^{*}$.

(i) The sheaves $R^{i} \nu_{X *}\left(\mathscr{L} \otimes \mathscr{O}_{\mathscr{X}_{\frac{o}{K}}}\right)$ and $R^{i} \nu_{X *}\left(\mathscr{L} \otimes \mathscr{J}_{X}\right)$ are almost isomorphic to quasi-coherent sheaves on $X_{\mathrm{et}}$, and are almost zero for $i>d$.

(ii) The map in $\widetilde{D}\left(\mathscr{O}_{X_{\bar{V}}}\right)$

$$
R \nu_{X *}\left(\mathscr{L} \otimes \mathscr{O}_{\mathscr{X}_{\bar{K}}}\right) \rightarrow R \mathscr{H} \operatorname{om}\left(R \nu_{X *}\left(\mathscr{L}^{*} \otimes \mathscr{J}_{X}\right), \Omega_{X / V}^{d} \otimes \bar{V} / p^{r}(-d)\right)
$$

induced by the trace map 6.13.1, is an isomorphism.

(iii) The trace map induces a canonical isomorphism in $\widetilde{D}(\bar{V})$

$$
R \Gamma\left(\mathscr{X}_{\bar{K}}^{o}, \mathscr{L} \otimes \mathscr{O}_{\mathscr{X}_{\frac{o}{K}}}\right) \rightarrow R \operatorname{Hom}\left(R \Gamma\left(\mathscr{X}_{\bar{K}}^{o}, \mathscr{L}^{*} \otimes \mathscr{J}_{X}\right), \bar{V} / p^{r} \bar{V}(-d)[-2 d]\right)
$$


Proof. Statement (i) follows from the same argument giving 6.13, and this argument combined with 4.11 also gives (ii). For (iii), note that by Serre duality and the fact that the complex $R \nu_{X *}\left(\mathscr{L}^{*} \otimes \mathscr{J}_{X}\right)$ is almost isomorphic to a bounded complex with coherent cohomology sheaves, the canonical pairing

$$
R \operatorname{Hom}\left(R \nu_{X *}\left(\mathscr{L}^{*} \otimes \mathscr{J}_{X}\right), \Omega_{X / V}^{d} \otimes \bar{V} / p^{r}\right) \otimes R \Gamma R \nu_{X *}\left(\mathscr{L}^{*} \otimes \mathscr{J}_{X}\right) \rightarrow \bar{V} / p^{r} \bar{V}
$$

is perfect. Now by (ii) we have

$$
R \operatorname{Hom}\left(R \nu_{X *}\left(\mathscr{L}^{*} \otimes \mathscr{J}_{X}\right), \Omega_{X / V}^{d} \otimes \bar{V} / p^{r}\right) \simeq R \Gamma\left(\mathscr{L} \otimes \mathscr{O}_{\mathscr{X}_{\bar{K}}}\right)
$$

which implies (iii).

By 6.5. the natural map

$$
H^{*}\left(\mathscr{X}_{\bar{K}}^{o}, \mathscr{L}\right) \rightarrow H^{*}\left(X_{\bar{K}, \text { et }}^{o}, L\right)
$$

is an isomorphism. The map of sheaves $\mathscr{L} \rightarrow \mathscr{L} \otimes \mathscr{O}_{\mathscr{X}_{\frac{o}{K}}}$ therefore induces a map on cohomology

$$
H^{*}\left(X_{\bar{K}, \mathrm{et}}^{o}, L\right) \otimes \bar{V} \rightarrow H^{*}\left(\mathscr{X}_{\bar{K}}^{o}, \mathscr{L} \otimes \mathscr{O}_{\mathscr{X}_{\bar{K}}^{o}}\right) .
$$

The following theorem will be proven in section 8 .

THEOREM 6.16. The morphism 6.15.3 is an isomorphism. In addition there is a canonical almost isomorphism

$$
H_{c}^{*}\left(X_{\bar{K}, \mathrm{et}}^{o}, L\right) \otimes \bar{V} \simeq H^{*}\left(\mathscr{X}_{\bar{K}}^{o}, \mathscr{L} \otimes \mathscr{J}_{X}\right),
$$

and these isomorphisms are compatible with the pairing 6.15.1 and the usual Poincaré duality for étale cohomology.

6.17. The isomorphism 6.15.1 can also be generalized to a statement with partial compact supports (this generalization is in fact crucial for the proof). Suppose given a decomposition $D=E \cup F$, where $E$ and $F$ are divisors on $X$ with no common irreducible component. Define $\mathscr{J}_{E} \subset \mathscr{O}_{\mathscr{X}_{\frac{O}{K}}}$ to be the sheaf of ideals which to any $(U, N) \in \mathscr{S}$ associates the ideal of $\left(E \times_{X} \bar{N}\right)_{\text {red }} \subset \bar{N}$, where $\bar{N}$ denotes the normalization of $U_{\bar{K}}$ in $N$. Note that multiplication induces a map $\mathscr{J}_{E} \otimes \mathscr{J}_{F} \rightarrow \mathscr{J}_{X}$ and hence using Poincaré duality we obtain for any locally constant sheaf of $\mathbb{Z} / p^{r}$ modules $L$ a map

$$
R \Gamma\left(\mathscr{X}_{\bar{K}}^{o}, \mathscr{L} \otimes \mathscr{J}_{E}\right) \rightarrow R \operatorname{Hom}\left(R \Gamma\left(\mathscr{X}_{\bar{K}}^{o}, \mathscr{L}^{*} \otimes \mathscr{J}_{F}\right), \bar{V} / p^{r} \bar{V}(-d)[-2 d]\right) .
$$

By the same argument used to prove 6.15 (iii) this map is an isomorphism.

\section{Computing compactly supported cohomology using Galois cohomology}

Before proving 6.16, we make in this section some general comments about how to compute compactly supported cohomology using Galois cohomology. These observations will only be used in the proof of 6.16 and can be omitted by the reader who skips this proof.

Throughout this section $\Lambda$ denotes the $\operatorname{ring} \mathbb{Z} / p^{n}$ for some $n$, and we consider $\Lambda$-modules unless otherwise stated. 
7.1. Let $Y$ be a smooth $\bar{K}$-scheme, $D=D_{1} \cup \cdots \cup D_{n}$ a divisor with simple normal crossings on $Y$, let $A \subset\{1, \ldots, n\}$ be a subset, and let $D_{A} \subset Y$ denote the intersection $\cap_{a \in A} D_{A}$. Let $D_{A}^{o}$ denote the complement in $D_{A}$ of $\cup_{i \notin A}\left(D_{i} \cap D_{A}\right)$, and let $j_{A}: D_{A}^{o} \hookrightarrow Y$ and $j: Y^{o} \hookrightarrow Y$ be the inclusions. Assume that $Y=\operatorname{Spec}(R)$ is affine, and that each $D_{A}^{o}$ is a $K(\pi, 1)$. We will further assume that the exists a formally étale morphism

$$
Y \rightarrow \operatorname{Spec}\left(\bar{K}\left[X_{1}, \ldots, X_{d}\right]\right)
$$

for some $d \geq 0$ such that $D$ is equal to the inverse image of $X_{1} \cdots X_{n}=0$ for some $n \leq d$.

7.2. We will also sometimes consider the following additional assumption (but will always state explicitly when we assume it):

(R): For every nonempty $A \subset\{1, \ldots, n\}$ there exists a retraction of the inclusion $D_{A} \hookrightarrow Y$

$$
r_{A}: Y \rightarrow D_{A}
$$

such that the inverse image of $D_{A}^{o}$ contains $Y^{o}$.

Example 7.3. The relevance of condition (R) for our purposes is the following. Let $X / V$ be a smooth proper scheme with $D \subset X$ a divisor with simple normal crossings relative to $V$, and let $\bar{x} \rightarrow X$ be a geometric point. Then $Y:=\operatorname{Spec}\left(\mathscr{O}_{X, \bar{x}} \otimes_{V} \bar{K}\right)$ with the pullback of the divisor $D$ satisfies condition (R).

7.4. For a finite étale morphism $Z^{o} \rightarrow Y^{o}$, let $Z \rightarrow Y$ be the normalization of $Y$ in $Z^{o}$, and let $Z_{A}^{o} \subset Z$ denote the maximal reduced closed subscheme of $D_{A}^{o} \times_{Y} Z$. We then have inclusions

$$
Z_{A}^{o} \stackrel{j_{A}^{Z}}{\longrightarrow} Z \stackrel{j^{Z}}{\longleftarrow} Z^{o}
$$

Lemma 7.5. The morphism $Z_{A}^{o} \rightarrow D_{A}^{o}$ is finite and étale.

Proof. The assertion is étale local on $Y$ so it suffices to consider the case when $Y$ is the spectrum of a strictly henselian local ring. In this case the result follows from Abhyankar's lemma [25, Appendice 1, 5.2].

Lemma 7.6. For any locally constant constructible sheaf of $\Lambda$-modules $L$ on $Y^{o}$ and $\nu \geq 0$ the sheaf $j_{A}^{*} R^{\nu} j_{*} L$ is locally constant constructible sheaf on $D_{A}^{o}$.

Proof. If $f: Y^{\prime o} \rightarrow Y^{o}$ is a finite étale morphism, then $f_{*} \Lambda$ is a locally constant constructible sheaf of $\Lambda$-modules on $Y^{o}$. Furthermore, for any locally constant constructible sheaf $L$ on $Y^{o}$ there exists a finite étale covering $f: Y^{\prime o} \rightarrow Y^{o}$ such that $f^{*} L$ is trivial. If we fix an isomorphism $f^{*} L \simeq \Lambda^{r}$ for some $r$ then the adjunction map $L \rightarrow f_{*} \Lambda^{r}$ is an inclusion. From this one deduces that for any locally constant constructible sheaf of $\Lambda$-modules $L$ on $Y^{o}$, there exists a resolution $L \rightarrow K^{\bullet}$ in the category of locally constant constructible sheaves of $\Lambda$-modules on $Y^{o}$ such that each $K^{n}$ is a direct sum of sheaves of the form $f_{*} \Lambda$ for some finite étale morphisms $f: Y^{\prime o} \rightarrow Y^{o}$. The spectral sequence corresponding to the "stupid filtration" on $K^{\bullet}$ yields

$$
E_{1}^{p q}=R^{q} j_{*} K^{p} \Longrightarrow R^{p+q} j_{*} L .
$$

From this it follows that it suffices to prove the lemma for $L=f_{*} \Lambda$ for some finite étale surjection $f: Y^{\prime o} \rightarrow Y^{o}$. 
In this case, let $\bar{f}: Y^{\prime} \rightarrow Y$ be the normalization of $Y$ in $Y^{\prime o}$, and let $D_{A}^{\prime o}$ (resp. $\left.D_{A}^{\prime}\right)$ denote the maximal reduced subscheme of $Y^{\prime} \times_{Y} D_{A}^{o}\left(\right.$ resp. $\left.Y^{\prime} \times_{Y} D_{A}\right)$ so that we have

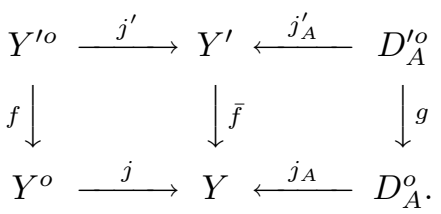

As in 7.5 the subscheme $D_{A}^{\prime} \subset Y^{\prime}$ is a divisor with normal crossings. We then have

$$
\begin{aligned}
j_{A}^{*} R^{\nu} j_{*} L & =j_{A}^{*} R^{\nu} j_{*}\left(f_{*} \Lambda\right) \\
& \simeq j_{A}^{*} R^{\nu} j_{*}\left(R f_{*} \Lambda\right) \\
& \simeq j_{A}^{*} R^{\nu} \bar{f}_{*}\left(R j_{*}^{\prime} \Lambda\right) \\
& \simeq g_{*} j_{A}^{\prime *} R^{\nu} j_{*}^{\prime} \Lambda,
\end{aligned}
$$

where the last isomorphism is by the proper base change theorem (note that the right square in 7.6 .2 is not cartesian but that $D_{A}^{\prime} \rightarrow Y^{\prime} \times_{Y} D_{A}$ is a closed immersion defined by a nilpotent ideal so that étale cohomology of a sheaf on $Y^{\prime} \times_{Y} D_{A}$ is equal to the étale cohomology of its pullback to $D_{A}^{\prime}$ ). It follows that it suffices to prove that $j_{A}^{\prime *} R^{\nu} j_{*}^{\prime} \Lambda$ is locally constant constructible on $D_{A}^{\prime o}$. This further reduces the proof to the case when $L=\Lambda$.

So let us finally prove the result for $L=\Lambda$. By replacing $Y$ by $Y-\cup_{i \notin A} D_{i}$ we may assume that in fact $A=\{1, \ldots, n\}$. Furthermore, the assertion is evidently étale local on $Y$ so we may assume that there is a smooth map

$$
\pi: Y \rightarrow \operatorname{Spec}\left(\bar{K}\left[T_{1}, \ldots, T_{n}\right]\right)
$$

such that $D_{i}$ is the inverse image of the zero locus of $T_{i}$ so that $D_{A}=\pi^{-1}(0)$. This morphism $\pi$ defines a morphism

$$
H^{\nu}\left(\mathbb{G}_{m}^{n}, \Lambda\right) \rightarrow j_{A}^{*} R^{\nu} j_{*} \Lambda
$$

which we claim is an isomorphism. To prove this we may replace $Y$ by $\operatorname{Spec}\left(\mathscr{O}_{Y, \bar{y}}\right)$ for a geometric point $\bar{y} \rightarrow D_{A}$. In this case $Y^{o}$ is a $K(\pi, 1)$ by 5.10 with fundamental group $\mathbb{Z}(1)^{n}$ so $\left(j_{A}^{*} R^{\nu} j_{*} \Lambda\right)_{\bar{y}}$ is isomorphic to the group cohomology $H^{\nu}\left(\mathbb{Z}(1)^{n}, L\right)$. On the other hand, using Künneth and the fact that $\mathbb{G}_{m}$ is a $K(\pi, 1)$ one sees that $H^{\nu}\left(\mathbb{G}_{m}^{n}, \Lambda\right)$ is also isomorphic to $H^{\nu}\left(\mathbb{Z}(1)^{n}, \Lambda\right)$ and under these identifications the map 7.6 .4 is the identity map.

7.7. Fix a geometric generic point $\operatorname{Spec}(\Omega) \rightarrow Y$, and let $\bar{R} \subset \Omega$ be the integral closure of $R$ in the compositum of all field extensions $k(Y) \subset L \subset \Omega$ for which the normalization of $R$ in $L$ is étale over $Y^{o}$. Let $\Delta$ denote the étale fundamental group of $Y^{o}$ with respect to the chosen base point so that $\Delta$ acts on $\operatorname{Spec}(\bar{R})$.

Let $A \subset\{1, \ldots, n\}$ be a subset and let $\bar{\eta} \rightarrow D_{A}^{o}$ be a geometric generic point. For any choice of lifting $\bar{\eta} \rightarrow \operatorname{Spec}(\bar{R})$ we obtain a decomposition group $H_{A} \subset \Delta$ defined to be the subgroup of elements fixing the image of $\bar{\eta}$. The subgroup $H_{A} \subset \Delta$ is closed. Let $\bar{R}_{A}$ be the quotient of $\bar{R}$ by the prime ideal corresponding to the image of $\bar{\eta}$. Then $\operatorname{Spec}\left(\bar{R}_{A}\right) \rightarrow D_{A}^{o}$ is an inverse limit of finite étale morphisms, and $H_{A}$ acts on the inverse limit. Let $I_{A} \subset H_{A}$ denote the subgroup of elements which act trivially on $\bar{R}_{A}$ (the inertia group). If $\Delta_{A}$ denotes the étale fundamental group of 
$D_{A}^{o}$ with respect to the base point $\bar{\eta}$, then the action of $H_{A} / I_{A}$ on $\bar{R}_{A}$ defines a surjection

$$
\Delta_{A} \rightarrow H_{A} / I_{A} .
$$

Lemma 7.8. If condition ( $R$ ) holds, then the homomorphism 7.7.1 is an isomorphism.

Proof. It suffices to show that any finite set with continuous $\Delta_{A}$-action is obtained by pullback from a set with continous $H_{A} / I_{A}$-action. This is clear for if $S$ is a set with continuous $\Delta_{A}$-action corresponding to an étale morphism $V \rightarrow D_{A}^{o}$, then $r_{A}^{*} V \rightarrow Y^{o}$ is an étale morphism, and the corresponding $H_{A} / I_{A^{-}}$set pulls back to $S$.

7.9. In terms of Galois representations, the sheaf $j_{A}^{*} j_{*} L$ can be described as follows. Let $M$ be the $\Delta$-representation corresponding to $L$. Then $j_{A}^{*} j_{*} L$ is the sheaf corresponding to the representation of $\Delta_{A}$ acting on $M^{I_{A}}$ via the surjection $\Delta_{A} \rightarrow H_{A} / I_{A}$. This implies in particular the following:

Corollary 7.10. Let $A \subset B \subset\{1, \ldots, n\}$ so that $D_{B} \subset D_{A}$. Then for any locally constant constructible sheaf of $\Lambda$-modules $L$ on $Y^{o}$ the natural map $j_{B}^{*} j_{*} L \rightarrow j_{B}^{*} j_{A *} j_{A}^{*} j_{*} L$ is an isomorphism.

7.11. Let $L$ be a locally constant sheaf of abelian groups on $Y_{\text {Fet }}^{o}$. For $A \subset$ $\{1, \ldots, n\}$ define a presheaf $\psi_{A}(L)$ on $\operatorname{Fet}\left(Y^{\circ}\right)$ by

$$
\left(Z^{o} \rightarrow Y^{o}\right) \mapsto \Gamma\left(Z_{A}^{o}, j_{A}^{Z *} j_{*}^{Z}\left(\left.L\right|_{Z^{o}}\right)\right) .
$$

In terms of Galois representations the presheaf $\psi_{A}(L)$ can be described as follows. Let $M$ be the $\Delta$-representation corresponding to $L$, and let $H_{A} \subset \Delta$ be the decomposition group of $D_{A}$ obtained by making suitable choices as in 7.7. Then we claim that $\psi_{A}(L)$ is the sheaf corresponding to the $\Delta$-representation

$$
\operatorname{Ind}_{H_{A}}^{\Delta}\left(\left.M\right|_{H_{A}}\right):=\operatorname{Hom}_{H_{A}}^{\mathrm{cts}}\left(\Delta,\left.M\right|_{H_{A}}\right) \text {. }
$$

Here $\operatorname{Ind}_{H_{A}}^{\Delta}(-)$ is the right adjoint to the restriction functor from $\Delta$-modules to $H_{A}$-modules.

To see this let $Z^{o} \rightarrow Y^{o}$ be a finite étale morphism corresponding to a $\Delta$-set $S$. The restriction of $S$ to $H_{A}$ decomposes into $H_{A}$-orbits

$$
\left.S\right|_{H_{A}}=\coprod_{i} S_{i}
$$

For each $i$ let $H_{i} \subset H_{A}$ denote the kernel of the map $H_{A} \rightarrow \operatorname{Aut}\left(S_{i}\right)$. We then have

$$
\begin{aligned}
\operatorname{Hom}_{\Delta}\left(S, \operatorname{Ind}_{H_{A}}^{\Delta}\left(\left.M\right|_{H_{A}}\right)\right) & \simeq \operatorname{Hom}_{H_{A}}(S, M) \\
& \simeq \prod_{i} \operatorname{Hom}_{H_{A}}\left(S_{i}, M\right) \\
& \simeq \prod_{i} M^{H_{i}} \\
& \simeq \Gamma\left(Z_{A}^{o}, j_{A}^{Z *} j_{*}^{Z} L\right) .
\end{aligned}
$$

Corollary 7.12. The presheaf $\psi_{A}(L)$ is a sheaf on $Y_{\text {Fet }}^{o}$.

COROLlary 7.13. The functor $\psi_{A}(-)$ is an exact functor on the category of sheaves on $Y_{\mathrm{Fet}}^{o}$. 
Proof. This is because $\operatorname{Ind}_{H_{A}}^{\Delta}(-)$ is an exact functor (this follows immediately from the definition).

Lemma 7.14. Let $\Delta$ be a profinite group and $D \subset \Delta$ a closed subgroup.

(i) Any injective $\Delta$-representation I is a direct summand of an induced module $\operatorname{Ind}_{D}^{\Delta}(N)$ with $N$ an injective $D$-representation.

(ii) For any injective $\Delta$-representation $I$ and $i>0$ the group $H^{i}\left(D,\left.I\right|_{D}\right)$ is zero.

Proof. Let $\widetilde{I}$ denote the restriction of $I$ to $D$ and choose an inclusion $\widetilde{I} \hookrightarrow$ $N$ with $N$ an injective $D$-representation. The adjunction map $I \rightarrow \operatorname{Ind}_{D}^{\Delta}(\widetilde{I}) \rightarrow$ $\operatorname{Ind}_{D}^{\Delta}(N)$ is then injective. Since $I$ is injective this inclusion $I \hookrightarrow \operatorname{Ind}_{D}^{\Delta}(N)$ is split which proves (i).

For (ii) note first that by (i) applied to the inclusion of the trivial group into $\Delta$, it suffices to consider the case of $I=\operatorname{Ind}^{\Delta}(N)$ (induction for the trivial subgroup $\{e\} \hookrightarrow \Delta$ ) for some injective abelian group $N$. Let $K_{n} \subset \Delta$ be a decreasing system of closed subgroups of finite index defining a base for the topology so that $\Delta=\lim _{\longleftarrow} \Delta / K_{n}$ and $D=\lim _{n} D /\left(D \cap K_{n}\right)$ (note since $D$ is closed in $\Delta$ such a system of subgroups $K_{n}$ exists). We then have

$$
\begin{aligned}
& H^{i}\left(D,\left.I\right|_{D}\right)=\lim _{n} H^{i}\left(D /\left(D \cap K_{n}\right), \operatorname{Hom}^{\text {cts }}(\Delta, N)^{D \cap K_{n}}\right) \\
& =\lim _{n} \underset{m}{\lim _{m}} H^{i}\left(D /\left(D \cap K_{n}\right), \operatorname{Hom}\left(\Delta / K_{m}, N\right)^{D \cap K_{n}}\right) .
\end{aligned}
$$

We can write this double limit as the single limit

$$
\underset{n}{\lim _{n}} H^{i}\left(D /\left(D \cap K_{n}\right), \operatorname{Hom}\left(\Delta / K_{n}, N\right)\right)
$$

The $D$-module $\operatorname{Hom}\left(\Delta / K_{n}, N\right)$ is a direct sum of modules $\operatorname{Hom}\left(D /\left(D \cap K_{n}\right), N\right)$ which is injective in the category of $D /\left(D \cap K_{n}\right)$-modules. From this it follows that the higher cohomology groups are zero.

COROLlary 7.15. Let $M$ be an injective object in the category of continuous representations of $\Delta$ on $\Lambda$-modules. Then the underlying $\Lambda$-module of $M$ is flat.

Proof. Taking $D=\{1\}$ in 7.14 we see that $M$ is a direct summand of $\operatorname{Ind}_{\{1\}}^{\Delta}(N)$ for some injective $\Lambda$-module $N$. Therefore it suffices to show that any injective $\Lambda$-module is flat over $\Lambda$. This is a standard consequence of the fact that $\Lambda$ is Gorenstein of dimension 0, which implies that $\Lambda$ is injective as a $\Lambda$-module (see for example [12, §21.2]). For the convenience of the reader let us recall the argument. It suffices to show that for any nonzero element $m \in M$ there exists an inclusion $i: \Lambda \hookrightarrow M$ such that $i(\Lambda)$ contains $m$. For since $\Lambda$ is injective such an inclusion $i$ is split so this will show that $M$ is isomorphic to filtered colimit of finitely generated free $\Lambda$-modules, and hence flat. Since $M$ is a $\Lambda$-module there exists a minimal integer $s$ such that $p^{s} m=0$, and this gives an inclusion $j: \mathbb{Z} /\left(p^{s}\right) \hookrightarrow M$ sending 1 to $m$. Since $M$ is injective there exists a dotted arrow $g$ filling in the diagram

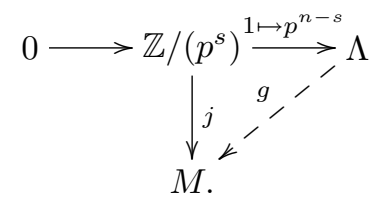


Because $j$ is an inclusion the map $g$ is also an inclusion, since by construction $g(1)$ has exact order $p^{n}$.

Lemma 7.16. With notation as in 7.7, let $M$ be an injective representation of $\Delta$. Then

(i)

$$
H^{i}\left(H_{A},\left.M\right|_{H_{A}}\right)=0
$$

for any $i>0$, and $H^{0}\left(H_{A},\left.M\right|_{H_{A}}\right)=H^{0}\left(\Delta_{A}, M^{I_{A}}\right)$.

(ii) If in addition condition $(R)$ holds then for any $i>0$ we have

$$
H^{i}\left(\Delta_{A}, M^{I_{A}}\right)=0 \text {. }
$$

Proof. Since $H_{A} \subset \Delta$ is closed the vanishing of $H^{i}\left(H_{A},\left.M\right|_{H_{A}}\right)$ follows from 7.14 The statement about $H^{0}$ is immediate.

For the vanishing of $H^{i}\left(\Delta_{A}, M^{I_{A}}\right)$ in the case when $(R)$ holds, note that the short exact sequence

$$
1 \rightarrow I_{A} \rightarrow H_{A} \rightarrow \Delta_{A} \rightarrow 1
$$

induces a spectral sequence

$$
E_{2}^{p q}=H^{p}\left(\Delta_{A}, H^{q}\left(I_{A}, M\right)\right) \Longrightarrow H^{p+q}\left(H_{A}, M\right) .
$$

Since $I_{A} \subset \Delta$ is also closed we have $H^{q}\left(I_{A}, M\right)=0$ for $q>0$ by 7.14 , and therefore there is a canonical isomorphism

$$
H^{p}\left(\Delta_{A}, M^{I_{A}}\right) \simeq H^{p}\left(H_{A}, M\right) .
$$

From this (ii) follows.

We can in fact sharpen the above lemma as follows.

Lemma 7.17. Let $\Delta$ be a profinite group. A filtering direct limit of injective continuous $\Delta$-representations is an injective continuous $\Delta$-representation.

Proof. Let $I=\underline{\lim } I_{\lambda}$ with each $I_{\lambda}$ an injective $\Delta$-representation. Consider an inclusion of $\Delta$-representations $N \hookrightarrow M$ and a map $\rho: N \rightarrow I$. We need to show that $\rho$ extends to a morphism $M \rightarrow I$. For this write $M=\lim M_{k}$ with each $M_{k}$

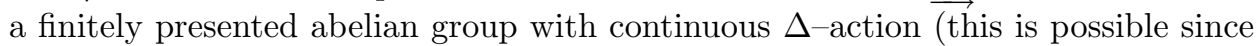
the action is continuous). Let $N_{k}$ denote $N \cap M_{k}$. We then inductively extend the maps $\rho_{k}: N_{k} \rightarrow I$ obtained from $\rho$.

For this fix some $k_{0}$. The map $N_{k_{0}} \rightarrow I$ factors through some $I_{\lambda}$ since $N_{k_{0}}$ is finitely presented and therefore there exists an extension $\tilde{\rho}_{k_{0}}: M_{k_{0}} \rightarrow I$.

Now assume that $\tilde{\rho}_{k}: M_{k} \rightarrow I$ has been constructed. By the definition of $N_{k}$ the map

$$
N_{k+1} \oplus_{N_{k}} M_{k} \rightarrow M_{k+1}
$$

is injective. Using the above argument applied to the map

$$
\rho_{k+1} \oplus \tilde{\rho}_{k}: N_{k+1} \oplus_{N_{k}} M_{k} \rightarrow I
$$

we then obtain the extension $\tilde{\rho}_{k+1}$.

Lemma 7.18. Let $\Delta$ be a profinite group and let $D \subset \Delta$ be a closed subgroup. If $M$ is an injective $\Delta$-representation then $\left.M\right|_{D}$ is an injective $D$-representation. 
Proof. Write $\Delta=\lim \Delta / K_{n}$ and set $S_{n}=\Delta /\left(D, K_{n}\right)$. Then the set of cosets $S:=\Delta / D$ is equal to $\lim S_{n}$ (and in particular has the profinite topology). Choose a continuous section $\sigma$ of the projection $\Delta \rightarrow \Delta / D$. Then for any continuous $D$-representation $N$ we have an isomorphism of $D$-representations

$$
\left.\operatorname{Hom}_{D}^{\text {cts }}(\Delta, N)\right|_{D} \rightarrow \operatorname{Hom}^{\text {cts }}(S, N), \quad(f: \Delta \rightarrow N) \mapsto(s \mapsto f(\sigma(s))) .
$$

Since

$$
\operatorname{Hom}^{\text {cts }}(S, N)=\underset{n}{\lim } \operatorname{Hom}\left(S_{n}, N\right)
$$

this, together with 7.17 proves the result in the case when $M=\operatorname{Ind}_{D}^{\Delta}(N)$ for some injective $D$-representation $N$. For the general case, note that by 7.14 any injective $M$ is a direct summand of a representation $\operatorname{Ind}_{D}^{\Delta}(N)$ with $N$ an injective $D$-representation.

Lemma 7.19. Let $\Delta$ be a profinite group and let $D \subset \Delta$ be a closed subgroup. Assume that $D \subset \Delta$ is normal, and let $G$ be the quotient. If $M$ is an injective $\Delta$-representation, then $M^{D}$ is an injective $G$-representation.

Proof. Immediate since the functor $M \mapsto M^{D}$ takes injectives to injectives.

Corollary 7.20. Let $\Delta$ be a profinite group, let $D_{1}, D_{2} \subset \Delta$ be two closed subgroups, and let $M$ be an injective $\Delta$-representation. Then for any $i>0$ we have

$$
H^{i}\left(D_{2},\left.\operatorname{Ind}_{D_{1}}^{\Delta}\left(\left.M\right|_{D_{1}}\right)\right|_{D_{2}}\right)=0 .
$$

7.21. Let $L$ be a locally constant sheaf of abelian groups on $Y_{\mathrm{et}}^{o}$. Define a complex of sheaves $\bar{\Psi}(L)$ on $\operatorname{Et}(Y)$ as follows. For $r \geq 0$ set

$$
\bar{\Psi}(L)^{r}:=\oplus_{A \subset\{1, \ldots, n\},|A|=r} j_{A *} j_{A}^{*} j_{*} L .
$$

For $1 \leq j \leq r$ and $A=\left\{i_{1}<i_{2}<\cdots<i_{r}\right\} \subset\{1, \ldots, n\}$ let $A_{j}$ denote the set $\left\{i_{1}, i_{2}, \ldots, \hat{i}_{j}, i_{j+1}, \ldots, i_{r}\right\}$ so that $A_{j} \subset A$. Using 7.10 we then have a map

$$
\partial_{j}: j_{A_{j} *} j_{A_{j}}^{*} j_{*} L \rightarrow j_{A *} j_{A}^{*} j_{A_{j} *} j_{A_{j}}^{*} j_{*} L \simeq j_{A *} j_{A}^{*} j_{*} L .
$$

Taking the sum of these maps we get a morphism

$$
\partial_{j}: \bar{\Psi}(L)^{r-1} \rightarrow \bar{\Psi}(L)^{r} .
$$

Define

$$
\partial: \bar{\Psi}(L)^{r-1} \rightarrow \bar{\Psi}(L)^{r}
$$

to be the alternating sum $\sum_{j=1}^{r+1}(-1)^{j} \partial_{j}$. One verifies immediately that $\partial^{2}=0$ so we get a complex $\bar{\Psi}(L)$ of sheaves on $\operatorname{Et}(Y)$.

We have $\bar{\Psi}(L)^{0}=j_{*} L$ and the composite

$$
j_{!} L \longrightarrow j_{*} L=\bar{\Psi}(L)^{0} \stackrel{\partial}{\longrightarrow} \bar{\Psi}(L)^{1}
$$

is zero.

Lemma 7.22. The map of complexes of sheaves

$$
j_{!} L \rightarrow \bar{\Psi}(L)
$$

is a quasi-isomorphism. 
Proof. It suffices to show that for any geometric point $\bar{y} \rightarrow Y$ the map of complexes of abelian groups

$$
\left(j_{!} L\right)_{\bar{y}} \rightarrow \bar{\Psi}(L)_{\bar{y}}
$$

is a quasi-isomorphism. If $\bar{y}$ maps to $Y^{o}$ this is immediate. If the image of $\bar{y}$ is in the boundary, the ring $\mathscr{O}_{Y, \bar{y}}$ is isomorphic to the strict henselization of the ring $K\left[X_{1}, \ldots, X_{d}\right]$ at the origin and $D$ is the zero locus of $X_{1} \cdots X_{r}$ for some $r \leq d$. In this case the fundamental group $\Delta$ of $\operatorname{Spec}\left(\mathscr{O}_{Y, \bar{y}}\right)^{o}$ is isomorphic to $\mathbb{Z}(1)^{r}$. If $M$ denotes the representation of $\Delta$ corresponding to $L$, then it follows from the construction that $\bar{\Psi}(L)_{\bar{y}}$ is isomorphic to the complex

$$
M^{\Delta} \otimes\left(\mathbb{Z} \rightarrow \oplus_{1 \leq i \leq r} \mathbb{Z} \rightarrow \oplus_{1 \leq i_{1}<i_{2} \leq r} \mathbb{Z} \rightarrow \cdots\right),
$$

where

$$
\oplus_{1 \leq i \leq r} \mathbb{Z} \rightarrow \oplus_{1 \leq i_{1}<i_{2} \leq r} \mathbb{Z} \rightarrow \cdots
$$

is the acyclic complex computing the Cech cohomology of $\operatorname{Spec}(\mathbb{Z})$ with respect to the covering

$$
\coprod_{i=1}^{r} \operatorname{Spec}(\mathbb{Z}) \rightarrow \operatorname{Spec}(\mathbb{Z})
$$

Lemma 7.23. Let $L$ be an injective $\Lambda$-module in $Y_{\text {Fet }}^{o}$. For any subset $A \subset$ $\{1, \ldots, n\}$ the natural map $j_{A *} j_{A}^{*} j_{*} L \rightarrow R j_{A *} j_{A}^{*} j_{*} L$ is an isomorphism.

Proof. Let $\bar{y} \rightarrow Y$ be a geometric point, and let $\widetilde{Y}$ denote $\operatorname{Spec}\left(\mathscr{O}_{Y, \bar{y}}\right)$. Then it suffices to show that for any $\nu>0$ we have

$$
H^{\nu}\left(\widetilde{Y}_{A}^{o}, j_{A}^{*} j_{*} L\right)=0 .
$$

The existence of the map 7.1.1 ensures that the morphism of fundamental groups $\pi_{1}\left(\widetilde{Y}^{o}\right) \rightarrow \pi_{1}\left(Y^{o}\right)$ (obtained by choosing suitable base points) is injective. Using 7.18 this implies that it suffices to prove the lemma with $Y$ replaced by $\tilde{Y}$. In this case $j_{A}^{*} j_{*} L$ is given by an injective representation of $\pi_{1}\left(Y_{A}^{o}\right)$ which implies the lemma.

LEmma 7.24. Let $L$ be an injective $\Lambda$-module in $Y_{\mathrm{Fet}}^{o}$ and assume condition $(R)$ holds. Then for any $i>0$ and any integer $r$ the group $H^{i}\left(Y_{\mathrm{et}}, \bar{\Psi}(L)^{r}\right)$ is zero.

Proof. For any $A \subset\{1, \ldots, n\}$ of size $r$ we have by 7.23

$$
H^{i}\left(Y_{\mathrm{et}}, j_{A *} j_{A}^{*} j_{*} L\right)=H^{i}\left(D_{A, \mathrm{et}}^{o}, j_{A}^{*} j_{*} L\right),
$$

which since $D_{A}^{o}$ is a $K(\pi, 1)$ and $j_{A}^{*} j_{*} L$ is locally constant is isomorphic to

$$
H^{i}\left(D_{A, \mathrm{Fet}}^{o}, j_{A}^{*} j_{*} L\right) .
$$

In the notation of 7.7, if $M$ is the $\Delta$-representation corresponding to $L$ we then want

$$
H^{i}\left(\Delta_{A}, M^{I_{A}}\right)=0
$$

for $i>0$. This follows from 7.16 
7.25. It follows that for a locally constant constructible sheaf $L$ of $\Lambda$-modules on $Y^{o}$, we can compute $H^{*}\left(Y_{\text {et }}, j_{!} L\right)$ as follows. Choose an injective resolution $L \rightarrow I^{\bullet}$ in the category of $\Lambda$-modules in $Y_{\text {Fet }}^{o}$, and let $\bar{\Psi}\left(I^{\bullet}\right)$ be the complex on $Y_{\text {et }}$ obtained by taking the total complex of the double complex obtained by applying $\bar{\Psi}(-)$ to each $I^{p}$. We then have a commutative diagram

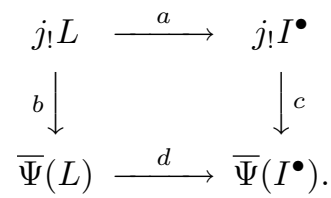

Since $j$ ! is an exact functor the morphism $a$ is a quasi-isomorphism, and $b$ and $c$ are quasi-isomorphisms by 7.22 . It follows that $d$ is also a quasi-isomorphism. We then have a map

$$
\Gamma\left(Y_{\text {et }}, \bar{\Psi}\left(I^{\bullet}\right)\right) \rightarrow R \Gamma\left(Y_{\text {et }}, j_{!} L\right)
$$

which by 7.24 is an isomorphism if $(\mathrm{R})$ holds.

7.26. For a sheaf of $\Lambda$-modules $L$ in $Y_{\text {Fet }}^{o}$ we can also define a complex $\Psi(L)$ in $Y_{\text {Fet }}^{o}$ as follows. We define

$$
\Psi(L)^{r}:=\bigoplus_{A \subset\{1, \ldots, n\},|A|=r} \psi_{A}(L),
$$

where $\psi_{A}(L)$ is defined as in 7.11 .

If $A \subset B \subset\{1, \ldots, n\}$, then there is a natural map

$$
\psi_{A}(L) \rightarrow \psi_{B}(L) .
$$

Indeed for any $Z^{o} \rightarrow Y^{o}$ with inclusions

$$
j^{Z}: Z^{o} \hookrightarrow Z, \quad j_{A}^{Z}: Z_{A}^{o} \hookrightarrow Z \text { etc. }
$$

we have a map

$$
\psi_{A}(L)\left(Z^{o}\right)=j_{A *}^{Z} j_{A}^{Z *} j_{*}^{Z} L(Z) \rightarrow j_{B}^{*} j_{A *}^{Z} j_{A}^{Z *} j_{*}^{Z} L\left(Z_{B}^{o}\right)=j_{B}^{Z *} j_{*}^{Z} L\left(Z_{B}^{o}\right)=\psi_{B}(L)\left(Z^{o}\right),
$$

where the second to last isomorphism is by 7.10 . Taking the alternating sum as in 7.21 we obtain a map

$$
\Psi(L)^{r-1} \rightarrow \Psi(L)^{r}
$$

and we write $\Psi(L)^{\bullet}$ for the resulting complex of sheaves on $Y_{\mathrm{Fet}}^{o}$.

We can compute $R \Gamma\left(Y_{\mathrm{Fet}}^{o}, \Psi(L)^{\bullet}\right)$ as follows. Let $L \rightarrow I^{\bullet}$ be an injective resolution in the category of $\Lambda$-modules in $Y_{\mathrm{Fet}}^{o}$, and let $\Psi\left(I^{\bullet}\right)$ be the total complex of the double complex obtained by applying $\Psi(-)$ to each $I^{p}$. By 7.13 the functor $\Psi(-)^{r}$ is exact, so we have a quasi-isomorphism

$$
\Psi(L)^{\bullet} \rightarrow \Psi\left(I^{\bullet}\right) .
$$

On the other hand, it follows from 7.16 that for any integer $i>0$ we have $H^{i}\left(Y_{\mathrm{Fet}}^{o}, \Psi\left(I^{\bullet}\right)^{r}\right)=0$. Therefore we obtain an isomorphism

$$
R \Gamma\left(Y_{\mathrm{Fet}}^{o}, \Psi\left(I^{\bullet}\right)\right) \simeq \Gamma\left(Y_{\mathrm{Fet}}^{o}, \Psi\left(I^{\bullet}\right)\right),
$$

and hence also an isomorphism

$$
R \Gamma\left(Y_{\mathrm{Fet}}^{o}, \Psi(L)\right) \simeq \Gamma\left(Y_{\mathrm{Fet}}^{o}, \Psi\left(I^{\bullet}\right)\right) .
$$


Now observe that by the definition of the sheaves $\psi_{A}(L)$ we have

$$
\Gamma\left(Y_{\mathrm{Fet}}^{o}, \psi_{A}\left(I^{p}\right)\right)=\Gamma\left(D_{A}^{o}, j_{A}^{*} j_{*} I^{p}\right) .
$$

It follows that

$$
\Gamma\left(Y_{\mathrm{Fet}}^{o}, \Psi\left(I^{\bullet}\right)\right)=\Gamma\left(Y_{\mathrm{et}}, \bar{\Psi}\left(I^{\bullet}\right)\right) .
$$

Combining 7.25 .2 and 7.26 .8 we obtain the following:

THEOREM 7.27. For any locally constant constructible sheaf $L$ in $Y_{\mathrm{Fet}}^{o}$, there is a canonical morphism

$$
R \Gamma\left(Y_{\mathrm{Fet}}^{o}, \Psi(L)\right) \rightarrow R \Gamma\left(Y_{\mathrm{et}}, j_{!} L\right),
$$

which is an isomorphism if condition $(R)$ holds.

REMARK 7.28. In the above we have chosen an injective resolution of $L$. One verifies immediately that the morphism in 7.27 does not depend on this choice.

REMARK 7.29. The preceding constructions are compatible with finite étale base change $Y^{\prime} \rightarrow Y$. In particular, if $a: Y_{\mathrm{Fet}}^{o} \rightarrow Y_{\mathrm{Fet}}$ and $b: Y_{\mathrm{et}} \rightarrow Y_{\mathrm{Fet}}$ are the natural morphisms of topoi, then $\Psi\left(I^{\bullet}\right)$ (resp. $\bar{\Psi}\left(I^{\bullet}\right)$ ) is a complex of sheaves acyclic for $a_{*}\left(\right.$ resp. $\left.b_{*}\right)$ and there is a canonical isomorphism

$$
a_{*} \Psi\left(I^{\bullet}\right) \simeq b_{*} \bar{\Psi}\left(I^{\bullet}\right)
$$

which induces the morphism 7.27.1 by applying $R \Gamma$.

7.30. Let $i: Z \subset Y$ be a smooth divisor meeting $D$ transversally, and let $Z^{o}$ denote $Z \times_{Y} Y^{o}$. Assume that $Z^{o}$ is a $K(\pi, 1)$ and that there is a retraction $r: Y^{o} \rightarrow Z^{o}$ of the inclusion $Z^{o} \hookrightarrow Y^{o}$. Then for any geometric point $\bar{y} \rightarrow Z^{o}$ the identity map on $\pi_{1}\left(Z^{o}, \bar{y}\right)$ factors through the map $\pi_{1}\left(Z^{o}, \bar{y}\right) \rightarrow \pi_{1}\left(Y^{o}, \bar{y}\right)$. In particular, the map $\pi_{1}\left(Z^{o}, \bar{y}\right) \rightarrow \pi_{1}\left(Y^{o}, \bar{y}\right)$ is injective and identifies $\pi_{1}\left(Z^{o}, \bar{y}\right)$ with a closed subgroup of $\pi_{1}\left(Y^{o}, \bar{y}\right)$.

Corollary 7.31. Let $L$ be an injective sheaf on $Y_{\mathrm{Fet}}^{o}$ and assume condition (R) holds. Then for any $A \subset\{1, \ldots, n\}$ the sheaf $i^{*} \Psi_{A}(L)$ on $Z_{\mathrm{Fet}}^{o}$ is acyclic for the global section functor.

Proof. If $\Delta$ denotes the fundamental group of $Y^{o}$ and $\Delta_{Z}$ the fundamental group of $Z^{o}$ then we have an inclusion $\Delta_{Z} \subset \Delta$ (well-defined up to conjugation). We also have the decomposition group $H_{A} \subset \Delta$ (also well-defined up to conjugation) and if $M$ is the representation of $\Delta$ corresponding to $L$ then $\psi_{A}(L)$ corresponds to the representation $\operatorname{Ind}_{H_{A}}^{\Delta}(M)$. The corollary therefore follows from 7.20

COROLlary 7.32. Let $L$ be an injective sheaf on $Y_{\mathrm{Fet}}^{o}$ and assume condition (R) holds. Then for any subset $A \subset\{1, \ldots, n\}$ the sheaf $j_{A}^{*} j_{*} L$ corresponds to a injective representation of $\pi_{1}\left(D_{A}^{o}\right)$.

Proof. Let $\Delta$ denote the fundamental group of $Y^{o}$, let $H_{A} \subset \Delta$ denote the decomposition group, and let $I_{A} \subset H_{A}$ be the inertia group. We then have $H_{A} / I_{A}$ isomorphic to the fundamental group of $D_{A}^{o}$. If $M$ is the $\Delta$-representation corresponding to $L$, then $j_{A}^{*} j_{*} L$ corresponds to the representation $\left(\left.M\right|_{H_{A}}\right)^{I_{A}}$ of $H_{A} / I_{A}$. By 7.18 the $H_{A}-$ representation $\left.M\right|_{H_{A}}$ is injective, and therefore the corollary follows from 7.19 . 


\section{Proof of 6.16}

Let $\Lambda$ denote the ring $\mathbb{Z} / p^{n}$ for some $n$.

8.1. Note first the if $Y$ is another smooth proper $V$-scheme with a divisor $D_{Y} \subset Y$ with relative normal crossings, and if $f: Y \rightarrow X$ is a proper morphism such that $f^{-1}\left(D_{X}\right) \subset D_{Y}$, then there is an induced morphism of topoi

$$
f: \mathscr{Y}_{\bar{K}}^{o} \rightarrow \mathscr{X}_{\bar{K}}^{o}
$$

such that the diagram

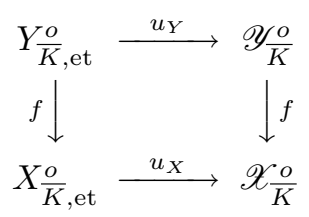

commutes.

8.2. We use this to reduce the proof of 6.16 to the case when $D$ has simple normal crossings as follows. By [33, 4.2.12] there exists a blow-up $f: Y \rightarrow X$ with support in $D$ such that $Y / V$ is smooth, $D_{Y}:=f^{-1}(D)$ is a divisor with simple normal crossings on $Y$ and the induced morphism of $\log$ schemes $f:\left(Y, M_{Y}\right) \rightarrow$ $\left(X, M_{X}\right)$ is log étale. This last observation implies that $f^{*} \Omega_{\left(X, M_{X}\right) / V}^{1} \simeq \Omega_{\left(Y, M_{Y}\right) / V}^{1}$. The morphism 8.1.1 induces morphisms

$$
H^{*}\left(\mathscr{X}_{\bar{K}}^{o}, \mathscr{L} \otimes \mathscr{O}_{\mathscr{X}_{\frac{o}{K}}}\right) \rightarrow H^{*}\left(\mathscr{Y}_{\bar{K}}^{o}, f^{*} \mathscr{L} \otimes \mathscr{O}_{\mathscr{Y} \frac{o}{K}}\right)
$$

and

$$
H^{*}\left(\mathscr{X}_{K}^{o}, \mathscr{L}^{*} \otimes \mathscr{J}_{X}\right) \rightarrow H^{*}\left(\mathscr{Y}_{K}^{o}, f^{*} \mathscr{L}^{*} \otimes \mathscr{J}_{Y}\right) .
$$

We claim that 8.2.1 and 8.2.2 are monomorphisms in $\bar{V}^{a}-$ Mod.

This follows from noting that by construction of the trace map, the diagrams

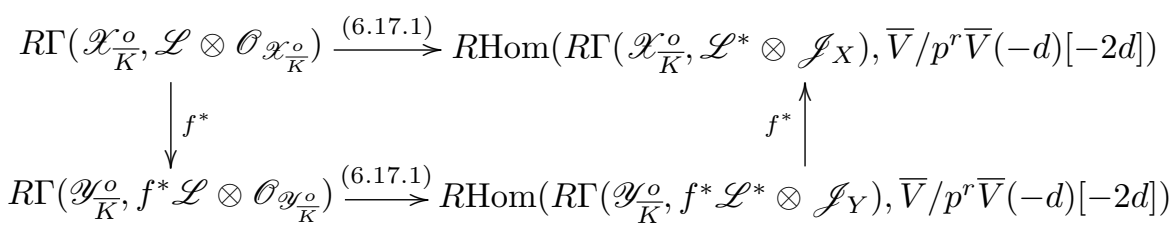

and

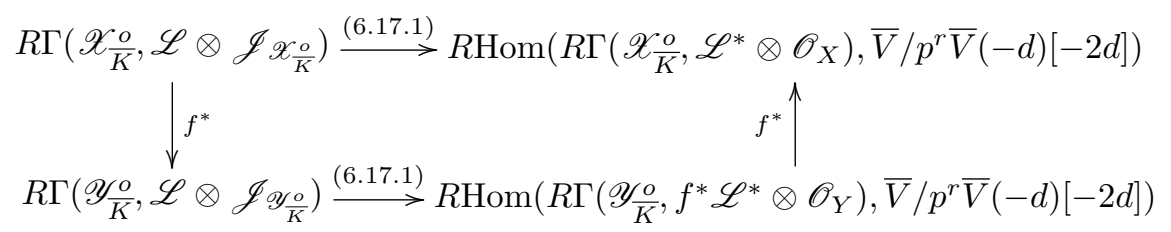

commute.

In particular, to prove 6.16 it suffices to show that the composite morphism

$$
H^{*}\left(X_{\bar{K}}^{o}, L\right) \rightarrow H^{*}\left(\mathscr{X}_{\bar{K}}^{o}, \mathscr{L} \otimes \mathscr{O}_{\mathscr{X}_{\bar{K}}^{o}}\right) \rightarrow H^{*}\left(\mathscr{Y}_{\bar{K}}^{o}, f^{*} \mathscr{L} \otimes \mathscr{O}_{\mathscr{Y}_{\bar{K}}}\right)
$$

is an isomorphism.

We may therefore assume that the divisor $D$ has simple normal crossings. Write $D=D_{1} \cup \cdots \cup D_{n}$ with each $D_{i} \subset X$ a smooth irreducible divisor. For a subset 
$A \subset\{1, \ldots, n\}$ we write $D_{A}$ for the intersection $\cap_{a \in A} D_{a}$. If $A=\emptyset$ then $D_{A}$ denotes $X$.

8.3. Let $\mathscr{S}$ denote the site defining $\mathscr{X}_{\bar{K}}^{o}$. For an abelian sheaf $\mathscr{L}$ in $\mathscr{X}_{\bar{K}}^{o}$ define a complex of presheaves $\Psi(\mathscr{L})$ on $\mathscr{S}$ by associating to any $U \in \operatorname{Et}(X)$ the complex of sheaves $\Psi\left(\mathscr{L}_{U}\right)$ on $U_{\bar{K}}^{o}$, Fet obtained by applying the construction in 7.26 to the sheaf $\mathscr{L}_{U}$ in $U_{\bar{K}}^{o}$, Fet. We denote by $\Psi^{a}(\mathscr{L})$ the associated complex of sheaves in $\mathscr{X}_{\bar{K}}^{o}$.

More generally if $\mathscr{L}^{\bullet}$ is a bounded below complex of abelian sheaves in $\mathscr{X}_{\bar{K}}^{o}$ we define $\Psi\left(\mathscr{L}^{\bullet}\right)$ to be the total complex of the bicomplex obtained by applying $\Psi(-)$ to each component of $\mathscr{L}^{\bullet}$. Passing to the associated sheaves we also define $\Psi^{a}\left(\mathscr{L}^{\bullet}\right)$. It follows from 7.13 that if $\mathscr{L}^{\bullet} \rightarrow \mathscr{M}^{\bullet}$ is a quasi-isomorphism, then the induced morphism

$$
\Psi^{a}\left(\mathscr{L}^{\bullet}\right) \rightarrow \Psi^{a}\left(\mathscr{M}^{\bullet}\right)
$$

is also a quasi-isomorphism.

8.4. Let $L$ be a locally constant constructible sheaf of $\Lambda$-modules in $X_{\bar{K}}^{o}$,et, and let $\mathscr{L}:=u_{X *} L$ denote the induced sheaf in $\mathscr{X}_{\bar{K}}^{o}$. Let $H R(X)^{\prime} \subset H R(X)$ be the full subcategory of hypercoverings $U$. $\rightarrow X$ such that for every $n$ the scheme $U_{n, \bar{K}}$ satisfies the assumptions of 7.1 . It follows from 5.11 that the subcategory $H R(X)^{\prime}$ is cofinal in $H R(X)$.

Let $\mathscr{L} \rightarrow I^{\bullet}$ be an injective resolution in $\mathscr{X}_{\bar{K}}^{o}$. For $U . \in H R(X)^{\prime}$ let

$$
\epsilon_{U}: \mathscr{X}_{\bar{K}}^{o} \rightarrow U_{\cdot, \bar{K}, \mathrm{Fet}}^{o}
$$

be the projection.

Lemma 8.5. For every $n$ the map

$$
\left.L\right|_{U_{n, \bar{K}, \mathrm{Fet}}^{o}}=\epsilon_{U_{n} *} \mathscr{L} \rightarrow \epsilon_{U_{n} *} I^{\bullet}
$$

is a quasi-isomorphism. Consequently, $\left.L\right|_{U^{\circ}, \bar{K}, \mathrm{Fet}} \rightarrow \epsilon_{U . *} I^{\bullet}$ is an injective resolution in the category of $\Lambda$-modules in the simplicial topos $U_{\cdot, \bar{K}, \mathrm{Fet}}^{o}$.

Proof. By 6.5 the natural map $\mathscr{L} \rightarrow R u_{X *} L$ is an isomorphism. It follows that if $\pi: U_{n, \bar{K}, \text { et }}^{o} \rightarrow U_{n, \bar{K}, \text { Fet }}^{o}$ is the projection then the right side of 8.5.1 computes $R \pi_{*} L$. The result then follows from the fact that $U_{n, \bar{K}}^{o}$ is a $K(\pi, 1)$.

8.6. We can also apply the $\bar{\Psi}(-)$-construction in 7.21 to the simplicial sheaves $\left.L\right|_{U^{\circ}, \bar{K}, \text { Fet }}$ and $\epsilon_{U, *} I^{\bullet}$. This gives complexes of sheaves $\Psi_{U .}(L)$ and $\bar{\Psi}\left(\epsilon_{U *} I^{\bullet}\right)$ on $U_{\bar{K}, \text { et }}$ and a commutative diagram of quasi-isomorphisms

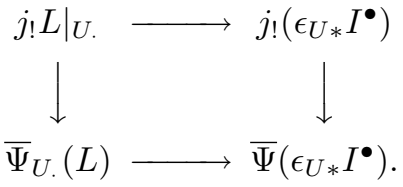

In particular we have

$$
R \Gamma\left(U_{\cdot, \bar{K}, \text { et }},\left.j_{!} L\right|_{U_{\cdot, \bar{K}}}\right) \simeq R \Gamma\left(U_{\cdot, \bar{K}, \text { et }}, \bar{\Psi}\left(\epsilon_{U *} I^{\bullet}\right)\right) .
$$

There is a canonical map

$$
\Gamma\left(U_{\cdot, \bar{K}, \mathrm{et}}, \bar{\Psi}\left(\epsilon_{U *} I^{\bullet}\right)\right) \rightarrow R \Gamma\left(U_{\cdot, \bar{K}, \mathrm{et}}, \bar{\Psi}\left(\epsilon_{U *} I^{\bullet}\right)\right)
$$


LEMma 8.7. The map

(8.7.1)

$\operatorname{hocolim}_{U . \in H R(X)^{\prime}} \Gamma\left(U_{\cdot, \bar{K}, \text { et }}, \bar{\Psi}\left(\epsilon_{U *} I^{\bullet}\right)\right) \rightarrow \operatorname{hocolim}_{U . \in H R(X)^{\prime}} R \Gamma\left(U_{\cdot, \bar{K}, \text { et }}, \bar{\Psi}\left(\epsilon_{U *} I^{\bullet}\right)\right)$

induced by 8.6 .3 is an isomorphism.

Proof. Write just $\bar{\Psi}\left(I^{\bullet}\right)$ for $\bar{\Psi}\left(\epsilon_{U *} I^{\bullet}\right)$. The map on cohomology obtained from 8.7.1 extends to a map of spectral sequences from

$$
E_{1}^{p q}=\underset{U .}{\lim _{\longrightarrow}} H^{q}\left(\Gamma\left(U_{p, \bar{K}}, \bar{\Psi}\left(I^{\bullet}\right)\right)\right) \Longrightarrow \underset{\lim _{.}}{\lim ^{p+q}}\left(\Gamma\left(U_{\cdot, \bar{K}}, \bar{\Psi}\left(I^{\bullet}\right)\right)\right)
$$

to

$$
E_{1}^{p q}=\underset{U .}{\lim _{U}} H^{q}\left(U_{p, \bar{K}}, \bar{\Psi}\left(I^{\bullet}\right)\right) \Longrightarrow \underset{U .}{\lim _{\longrightarrow}} H^{p+q}\left(U_{\cdot, \bar{K}}, \bar{\Psi}\left(I^{\bullet}\right)\right) .
$$

It follows that it suffices to show that for every $(p, q)$ the map

$$
\underset{U .}{\lim _{U}} H^{q}\left(\Gamma\left(U_{p, \bar{K}}, \bar{\Psi}\left(I^{\bullet}\right)\right)\right) \rightarrow \underset{U .}{\lim _{\longrightarrow}} H^{q}\left(U_{p, \bar{K}}, \bar{\Psi}\left(I^{\bullet}\right)\right)
$$

is an isomorphism. For this in turn it suffices to show that for every $(p, q)$ with $q>0$ and $j, r \in \mathbb{Z}$ we have

$$
\underset{U .}{\lim _{U}} H^{q}\left(U_{p, \bar{K}}, \bar{\Psi}\left(I^{j}\right)^{r}\right)=0 .
$$

To verify this it suffices by a standard limit argument to consider the case of the strict henselization of $X$ at a point. In this case condition (R) in 7.2 holds so the result follows from 7.24 .

8.8. By 7.26.10 we also have

$$
\Gamma\left(U_{\cdot, \bar{K}, \text { et }}, \bar{\Psi}\left(\epsilon_{U *} I^{\bullet}\right)\right)=\Gamma\left(U_{\cdot, \bar{K}, \text { Fet }}^{o}, \Psi\left(\epsilon_{U *} I^{\bullet}\right)\right)
$$

We then have

$$
\begin{aligned}
R \Gamma\left(\mathscr{X}_{\bar{K}}^{o}, \Psi^{a}(\mathscr{L})\right) & =R \Gamma\left(\mathscr{X}_{\bar{K}}^{o}, \Psi^{a}\left(I^{\bullet}\right)\right) \quad\left(\text { by exactness of } \Psi^{a}\right) \\
& =\operatorname{hocolim}_{U, \in H R(X)^{\prime}} R \Gamma\left(U_{\cdot, \bar{K}, \mathrm{Fet}}^{o}, \Psi\left(\epsilon_{U . *} I^{\bullet}\right)\right) \quad \text { by 6.11) } \\
& =\operatorname{hocolim}_{U, \in H R(X)^{\prime}} \Gamma\left(U_{\cdot, \bar{K}, \mathrm{Fet}}, \Psi\left(\epsilon_{U \cdot *} I^{\bullet}\right)\right) \quad \text { by 7.26.7) } \\
& =\operatorname{hocolim}_{U, \in H R(X)^{\prime}} \Gamma\left(U_{\cdot, \bar{K}, \mathrm{et}}, \bar{\Psi}\left(\epsilon_{U, *} I^{\bullet}\right)\right) \quad \text { by 8.8.1) } \\
& =\operatorname{hocolim}_{U, \in H R(X)^{\prime}} R \Gamma\left(U_{\cdot, \bar{K}, \mathrm{et}},\left.j_{!} L\right|_{U_{\bar{K}}}\right) \quad \text { by 8.7 and 8.6.2) } \\
& =R \Gamma\left(X_{\bar{K}}, j_{!} L\right) .
\end{aligned}
$$

Summarizing:

THEOREM 8.9. There is a canonical isomorphism

$$
H_{c}^{*}\left(X_{\bar{K}}^{o}, L\right) \simeq H^{*}\left(\mathscr{X}_{\bar{K}}^{o}, \Psi^{a}(\mathscr{L})\right)
$$

functorial in $L$.

REMARK 8.10. In the above we have chosen the injective resolution $\mathscr{L} \rightarrow I^{\bullet}$. A standard verification shows that the isomorphism in 8.9 is independent of this choice. 
8.11. In fact this isomorphism is even compatible with cup products. Let $\mathscr{L}$ be the sheaf on $\mathscr{X}_{\bar{K}}^{\circ}$ associated (by $u_{X *}$ ) to a locally constant constructible sheaf $L$ of projective $\Lambda$-modules on $X_{\bar{K}}^{o}$ and let $L^{*}$ be the dual of $L$ with corresponding sheaf $\mathscr{L}^{*}:=u_{X *} L^{*}$. Then it follows from the construction that there are canonical maps

$$
\mathscr{L} \otimes \Psi\left(\mathscr{L}^{*}\right) \rightarrow \Psi\left(\mathbb{Z} / p^{r}\right)
$$

and

$$
j_{*} L \otimes \bar{\Psi}\left(L^{*}\right) \rightarrow \bar{\Psi}\left(\mathbb{Z} / p^{r}\right)
$$

on $\mathscr{X}_{\bar{K}}^{o}$ and $X_{\bar{K}}$ respectively, which induce cup products

$$
\cup: H^{*}\left(\mathscr{X}_{\bar{K}}^{o}, \mathscr{L}\right) \times H^{*}\left(\mathscr{X}_{\bar{K}}^{o}, \Psi^{a}\left(\mathscr{L}^{*}\right)\right) \rightarrow H^{*}\left(\mathscr{X}_{\bar{K}}^{o}, \Psi^{a}\left(\mathbb{Z} / p^{r}\right)\right),
$$

and

$$
\cup: H^{*}\left(X_{\bar{K}}^{o}, L\right) \times H_{c}^{*}\left(X_{\bar{K}}^{o}, L^{*}\right) \rightarrow H_{c}^{*}\left(X_{\bar{K}}^{o}, \mathbb{Z} / p^{r}\right)
$$

and the isomorphisms obtained from 6.5 and 8.9 are compatible with these pairings.

8.12. There is also a finer variant of the above construction. Let $D=E \cup F$ be a decomposition of $D$ into two divisors, and let $\Psi_{E}(\mathscr{L}) \subset \Psi(\mathscr{L})$ denote the subcomplex obtained by taking in 8.3 only the sum over divisors lying in $E$ and intersections of such divisors. Then by the same argument used to prove 8.9 , one gets a canonical isomorphism in the derived category

$$
R \Gamma\left(\mathscr{X}_{\bar{K}}^{o}, \Psi_{E}^{a}(\mathscr{L})\right) \simeq R \Gamma\left((X-F)_{\bar{K}}, j_{!} L\right) .
$$

In what follows we denote the right side of this equation by $R \Gamma_{E, F}\left(X_{\bar{K}}^{o}, L\right)$ and by $H_{E, F}^{*}\left(X_{\bar{K}}^{o}, L\right)$ the corresponding cohomology groups (though of course these groups depend on the compactification $X$ of $X^{o}$ ).

These cohomology groups with partial compact support also satisfy Poincaré duality:

Proposition 8.13. With notation as above, there is a canonical isomorphism

$$
R \Gamma_{E, F}\left(X_{\bar{K}}^{o}, L\right) \rightarrow R \operatorname{Hom}\left(R \Gamma_{F, E}\left(X_{\bar{K}}^{o}, L^{*}\right), \Lambda(-d)[-2 d]\right) .
$$

Proof. Consider the commutative diagram

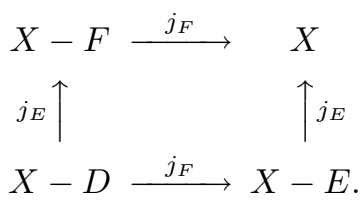

Lemma 8.14. For any locally constant constructible sheaf $H$ of $\Lambda$-modules on $(X-D)_{\bar{K}}$ there is a canonical isomorphism

$$
j_{E !} R j_{F *}(H) \rightarrow R j_{F *} j_{E !}(H) .
$$

Proof. Note first there there is a canonical map $j_{E !} j_{F *} \rightarrow j_{F *} j_{E !}$, induced by adjunction from the natural isomorphism $j_{E}^{*} j_{F *} j_{E !} \simeq j_{F *}$. By the universal property of derived functors we therefore obtain a morphism of functors

$$
j_{E !} R j_{F *} \rightarrow R j_{F *} j_{E !}
$$

This defines the morphism 8.14.1. To verify that it is an isomorphism for $H$ locally constant constructible, we may pass to the strict henselization of $X_{\bar{K}}$ at a geometric 
point. Let $d_{E}$ (resp. $d_{F}$ ) denote the number of components of $E$ (resp. $F$ ). Then the fundamental group $\Delta_{E}$ of $X-E$ is isomorphic to $\widehat{\mathbb{Z}}(1)^{d_{E}}$ and the fundamental group $\Delta_{F}$ of $X-F$ is isomorphic to $\widehat{\mathbb{Z}}(1)^{d_{F}}$, and the fundamental group $\Delta$ of $X-D$ is isomorphic to $\Delta_{E} \times \Delta_{F}$. By filtering $H$ first by subsheaves of the form $n H(n \in \mathbb{Z})$, and then further by irreducible subrepresentations the proof is then further reduced to the case when $H$ is of the form $H_{E} \otimes H_{F}$ for some representations $H_{E}$ (resp. $\left.H_{F}\right)$ of $\Delta_{E}$ (resp. $\left.\Delta_{F}\right)$. This reduces the proof the case when $X=\mathbb{A}^{d_{E}} \times \mathbb{A}^{d_{F}}$ for some integers $d_{E}$ and $d_{F}, X-E=\mathbb{G}_{m}^{d_{E}} \times \mathbb{A}^{d_{F}}, X-F=\mathbb{A}^{d_{E}} \times \mathbb{G}_{m}^{d_{F}}$, and $H$ is obtained from a sheaf $H_{E}$ on $\mathbb{G}_{m}^{d_{E}}$ tensored with a sheaf $H_{F}$ pulled back from $\mathbb{G}_{m}^{d_{F}}$. In this case the result follows from the Künneth formula [24, III, 1.6 .4 and $1.7 .1]$.

For a locally constant constructible sheaf $H$ of projective $\Lambda$-modules on $(X-$ $D)_{\bar{K}}$ we have

$$
R j_{F *} j_{E !}(H) \otimes^{\mathbb{L}} R j_{E *} j_{F !} H^{*} \simeq j_{!}\left(H \otimes H^{*}\right) .
$$

The trace map therefore induces a morphism

$$
R \Gamma\left(X_{\bar{K}}, R j_{F *} j_{E !}(H)\right) \otimes^{\mathbb{L}} R \Gamma\left(X_{\bar{K}}, R j_{E *} j_{F !}\left(H^{*}\right)\right) \rightarrow \Lambda(-d)[-2 d]
$$

which defines the map 8.13.1. To see that it is an isomorphism, one can either proceed by a local group cohomology computation, or as follows using local duality. Let $f: X_{\bar{K}} \rightarrow \operatorname{Spec}(\bar{K})$ momentarily denote the structure morphism, and let $\omega_{X_{\bar{K}}}:=f^{!} \Lambda \simeq \Lambda(-d)[-2 d]$ denote the dualizing complex [24, I]. Write $D_{X}(-)$ for the functor $R \mathscr{H}$ om $\left(-, \omega_{X_{\bar{K}}}\right)$ and $D_{\bar{K}}(-)$ for $R \operatorname{Hom}(-, \Lambda)$.

By duality 9, Th. Finitude, 4.3] and 8.14 above we then have

$$
\begin{aligned}
R j_{F *} j_{E !}(H) & \simeq j_{E !} R j_{F *}(H) \\
& \simeq D_{X} R j_{E *} D_{X-E} R j_{F *} D_{X-D}\left(D_{X-D}(H)\right) \\
& \simeq D_{X} R j_{E *} j_{F !}\left(D_{X-D}(H)\right) .
\end{aligned}
$$

Since $f$ is proper so that $R f_{!}=D_{\bar{K}} R f_{*} D_{X}$ is equal to $R f_{*}$, we get (8.14.5)

$$
D_{\bar{K}} R f_{*} R j_{F *} j_{E !}(H) \simeq R f_{*} R j_{E *} j_{F_{!}}\left(D_{X-D}(H)\right) \simeq R f_{*} R j_{E *} j_{F_{!}}\left(H^{*}\right)(-d)[-2 d] .
$$

8.15. Next we define another complex $\Phi\left(\mathscr{O}_{\mathscr{X}_{\bar{K}}}\right)$ on $\mathscr{X}_{\bar{K}}^{o}$.

For $A \subset\{1, \ldots, n\}$ the $V$-scheme $D_{A}$ is smooth, and the closed subscheme

$$
\bigcup_{i \notin A}\left(D_{i} \cap D_{A}\right) \subset D_{A}
$$

is a divisor with normal crossings in $D_{A}$. We can therefore apply the discussion in section 6 to $D_{A}$ with respect to the divisor 8.15.1 to get a topos $\mathscr{D}_{A, \bar{K}}^{o}$. We write $\mathscr{S}_{A}$ for the site defining $\mathscr{D}_{A, \bar{K}}^{o}$.

For an object $(U, N)$ of $\mathscr{S}$ (the site defining $\mathscr{X}_{\bar{K}}^{o}$ ), let $\bar{N} \rightarrow U_{\bar{K}}$ be the normalization of $U_{\bar{K}}$ in $N$, and let $\bar{N}_{A}^{O}$ denote the maximal reduced subscheme of the pullback of $\bar{N}$ to $D_{A}^{o} \times_{X} \bar{N}$. Then $\bar{N}_{A}^{o} \rightarrow D_{A, \bar{K}}^{o}$ is finite and étale by 7.5 , and therefore $\left(U_{A}, \bar{N}_{A}^{O}\right)$ is an object of the site $\mathscr{S}_{A}$. 
Define $\Phi\left(\mathscr{O}_{\mathscr{X}_{\frac{o}{K}}}\right)^{r}$ to be the presheaf

$$
(U, N) \mapsto \oplus_{A \subset\{1, \ldots, n\},|A|=r} \Gamma\left(\left(U_{A}, \bar{N}_{A}^{o}\right), \mathscr{O}_{\mathscr{D}_{A, \bar{K}}^{o}}\right),
$$

and let $\Phi^{a}\left(\mathscr{O}_{\mathscr{X}_{\frac{o}{K}}}\right)^{r}$ be the associated sheaf. We define a map

$$
d: \Phi^{a}\left(\mathscr{O}_{\mathscr{X}_{\frac{o}{K}}}\right)^{r} \rightarrow \Phi^{a}\left(\mathscr{O}_{\mathscr{X}_{\frac{o}{K}}}\right)^{r+1}
$$

as follows. Say $A=\left\{i_{1}, \ldots, i_{r+1}\right\}$ and for $1 \leq j \leq r+1$ let $A_{j}=\left\{i_{1}, \ldots, \hat{i}_{j}, \ldots, i_{r+1}\right\}$.

Let $(U, N) \in \mathscr{S}$ be an object with $U=\operatorname{Spec}(R)$ affine, and let $S$ denote the coordinate ring of the normalization of $U$ in $N$. If $I_{A} \subset R$ (resp. $I_{A_{j}} \subset R$ ) denotes the ideal of $D_{A}$ (resp. $\left.D_{A_{j}}\right)$, then $\Gamma\left(\left(U_{A}, \bar{N}_{A}^{o}\right), \widetilde{\mathscr{O}}_{\mathscr{D}_{A}^{o}}\right)$ is equal to the normalization of $\left(S / I_{A} S\right)_{\text {red }}$, and $\Gamma\left(\left(U_{A_{j}}, \bar{N}_{A_{j}}^{o}\right), \widetilde{\mathscr{O}}_{\mathscr{D}_{A_{j}}^{o}}\right)$ is equal to the normalization of $\left(S / I_{A_{j}} S\right)_{\text {red }}$ (here $\widetilde{\mathscr{O}}_{\mathscr{D}_{A}^{o}}$ and $\widetilde{\mathscr{O}}_{\mathscr{D}_{A j}^{o}}$ are as in 6.12. Since $D_{A} \subset D_{A_{j}}$ we have $I_{A_{j}} \subset I_{A}$, and hence there is a canonical map

$$
\partial_{j}: \Gamma\left(\left(U_{A_{j}}, \bar{N}_{A_{j}}^{o}\right), \widetilde{\mathscr{O}}_{\mathscr{D}_{A_{j}, \bar{K}}^{o}}\right)=\left(S / I_{A_{j}} S\right)^{\sim} \rightarrow\left(S / I_{A} S\right)^{\sim}=\Gamma\left(\left(U_{A}, \bar{N}_{A}^{o}\right), \widetilde{\mathscr{O}}_{\mathscr{D}_{A, \bar{K}}^{o}}\right) .
$$

Taking the alternating sums $\sum_{j=1}^{r+1}$ for various choices of $A$ and sheafifying we obtain the map 8.15.3. It follows immediately from the construction that $d^{2}=0$ so we get a complex denoted $\Phi^{a}\left(\mathscr{O}_{\mathscr{X}_{\frac{o}{K}}}\right)$.

Note that $\Phi^{a}\left(\mathscr{O}_{\mathscr{X}_{\frac{o}{K}}}\right)^{0}=\mathscr{O}_{\mathscr{X}_{\frac{o}{K}}}$. In particular, there is a canonical map $\mathscr{J}_{X} \rightarrow$ $\Phi^{a}\left(\mathscr{O}_{\mathscr{X}_{\frac{o}{K}}}\right)^{0}$ and it follows immediately from the above computations that $d\left(\mathscr{J}_{X}\right)=$ 0 . We therefore get a morphism of complexes

$$
\mathscr{J}_{X} \rightarrow \Phi^{a}\left(\mathscr{O}_{\mathscr{X}_{\frac{o}{K}}}\right)
$$

Similarly, for any integer $r \geq 1$ we get a complex $\Phi^{a}\left(\mathscr{O}_{\mathscr{X}_{\frac{o}{K}}} / p^{r}\right)$ by replacing $\mathscr{O}_{\mathscr{D}_{A, \bar{K}}^{o}}$ in the above with $\mathscr{O}_{\mathscr{D}_{A, \bar{K}}^{o}} / p^{r}$, and the map 8.15 .5 induces a morphism

$$
\mathscr{J}_{X} / p^{r} \rightarrow \Phi^{a}\left(\mathscr{O}_{\mathscr{X}_{\frac{o}{K}}} / p^{r}\right)
$$

Proposition 8.16. For every integer $r \geq 1$, the induced morphism

$$
R \nu_{X *} \mathscr{J}_{X} / p^{r} \rightarrow R \nu_{X *} \Phi^{a}\left(\mathscr{O}_{\mathscr{X}_{\frac{o}{K}}} / p^{r}\right)
$$

is an isomorphism in $\widetilde{D}\left(\mathscr{O}_{X_{\bar{V}}}\right)$.

Proof. By a standard reduction it suffices to consider the case when $X=$ $\operatorname{Spec}(R)$ is the spectrum of a strictly henselian local ring, and to prove the corresponding result for global cohomology groups. In this case by our assumptions each $D_{A}$ also has the same form and we can also apply the purity theorem 2.17 to $D_{A}$. Let $S=V\left[T_{1}, \ldots, T_{d}\right]$ and let $S_{\infty}$ be as in 2.16. For any integer $r$ and $1 \leq i_{1}<$ $i_{2}<\cdots<i_{r} \leq d$ let $S_{i_{1} \ldots i_{r}, \infty}$ denote $\left(S_{\infty} /\left(T_{i_{1}}, \ldots, T_{i_{r}}\right)\right)_{\text {red }}=\left(S /\left(T_{i_{1}}, \ldots, T_{i_{r}}\right)\right)_{\infty}$. Then by 3.10 and 6.9 the complex $R \nu_{X *} \Phi^{a}\left(\mathscr{O}_{\mathscr{X}_{\frac{o}{K}}} / p^{r}\right)$ is almost isomorphic to

$$
R \Gamma\left(\Delta_{\infty}, R / p^{r} \otimes_{S}\left(S_{\infty} \rightarrow \oplus_{i} S_{i, \infty} \rightarrow \oplus_{i_{1}<i_{2}} S_{i_{1} i_{2}, \infty} \rightarrow \cdots\right)\right),
$$

and as before

$$
R \nu_{X *} \mathscr{J}_{X} / p^{r} \simeq R \Gamma\left(\Delta_{\infty}, R /\left(p^{r}\right) \otimes_{S} J_{\infty}^{(S)}\right) \quad \text { (almost isomorphism). }
$$

It therefore suffices to show that the natural map

$$
J_{\infty}^{(S)} \rightarrow\left(S_{\infty} \rightarrow \oplus_{i} S_{i, \infty} \rightarrow \oplus_{i_{1}<i_{2}} S_{i_{1} i_{2}, \infty} \rightarrow \cdots\right)
$$


is a quasi-isomorphism (since this is a complex of $p$-torsion free modules this will also imply that it is a quasi-isomorphism modulo $p^{r}$ ). For this note that the complex on the right side is equal to the tensor product of the complexes obtained from each $V\left[T_{i}\right]$ and $J_{\infty}^{(S)}$ is equal to the tensor product of the complexes obtained from the ideal defining the origin in each $V\left[T_{i}\right]$. It therefore suffices to consider the case when $d=1$ in which case the result is immediate.

8.17. More generally for a subset $E \subset\{1, \ldots, n\}$ there is a complex $\Phi_{E}^{a}\left(\mathscr{O}_{\mathscr{X}_{\frac{o}{K}}}\right)$ obtained by summing only over subsets $A \subset E$ in 8.15 .2 . The same argument used above shows that there is a canonical morphism

$$
\mathscr{J}_{E} \rightarrow \Phi_{E}^{a}\left(\mathscr{O}_{\mathscr{X}_{K}^{o}}\right)
$$

which induces an almost isomorphism after dividing by $p^{r}$ and applying $R \nu_{X *}$.

8.18. Fix $A \subset\{1, \ldots, n\}$. The presheaf

$$
(U, N) \mapsto \Gamma\left(\bar{N}_{A}^{o}, \widetilde{\mathscr{O}}_{\mathscr{D}_{A}^{o}}\right)
$$

can be described Galois-theoretically as follows. Let $U \rightarrow X$ be an étale morphism with $U=\operatorname{Spec}(R)$ affine, and assume that $U_{\bar{K}}^{o}$ satisfies the assumptions in 7.1 . Define groups $\Delta, H_{A}, I_{A}$ etc. as in 7.7. Let $R_{A}$ denote the coordinate ring of $D_{A}$. Let $\bar{R}_{A}$ denote the integral closure of $R_{A}$ in the maximal subextension of the chosen geometric generic point of $D_{A, \bar{K}}^{o}$ which is unramified over $D_{A, \bar{K}}^{o}$. Then $\bar{R}_{A}$ is a continuous representation of $\Delta_{A}$ and $\widetilde{\mathscr{O}}_{\mathscr{D}_{A}^{\circ}}$ is the corresponding presheaf on $\mathscr{S}_{A}$ (defined as in 8.15. The restriction of the presheaf 8.18.1 to $U_{\bar{K} \text {, Fet }}^{o}$ is then the sheaf corresponding to the induced representation $\operatorname{Ind}_{H_{A}}^{\Delta}\left(\vec{R}_{A}\right)$. Note in particular that by the projection formula for any continuous $\Delta$-representation $M$ there is a canonical isomorphism

$$
M \otimes \operatorname{Ind}_{H_{A}}^{\Delta}\left(\bar{R}_{A}\right) \rightarrow \operatorname{Ind}_{H_{A}}^{\Delta}\left(\left.M\right|_{\Delta_{A}} \otimes \bar{R}_{A}\right)
$$

This implies in particular that for any sheaf $\mathscr{L}$ in $\mathscr{X}_{\frac{o}{K}}$ there is a canonical map of complexes in $\mathscr{X}_{\bar{K}}^{o}$

$$
\Psi^{a}(\mathscr{L}) \rightarrow \mathscr{L} \otimes \Phi^{a}\left(\mathscr{O}_{\mathscr{X}_{\frac{o}{K}}}\right)
$$

and similarly for the complexes defining cohomology with partial compact support.

8.19. Fix now a decomposition $D=E \cup F$. Let $L$ be a locally constant constructible sheaf of flat $\mathbb{Z} / p^{r}$-modules on $X_{\bar{K}}^{o}$ and set $\mathscr{L}:=u_{X *} L$. We then get a morphism

$$
\mathscr{L} \otimes \mathscr{J}_{E} \rightarrow \mathscr{L} \otimes \Phi_{E}^{a}\left(\mathscr{O}_{\mathscr{X}_{\frac{o}{K}}^{o}}\right)
$$

which induces an almost quasi-isomorphism after applying $R \nu_{X *}$. Combining this with 8.18 .3 we obtain a morphism in $\bar{V}^{a}-\operatorname{Mod}$

$$
R \Gamma_{E, F}\left(X_{\bar{K}}^{o}, L\right) \simeq R \Gamma\left(\mathscr{X}_{\bar{K}}^{o}, \Psi_{E}^{a}(\mathscr{L})\right) \rightarrow R \Gamma\left(\mathscr{X}_{\bar{K}}^{o}, \mathscr{L} \otimes \mathscr{J}_{E}\right) .
$$

It follows from the construction that the resulting diagram

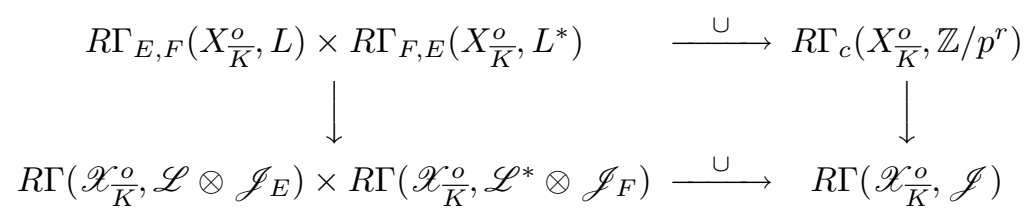


commutes.

This defines the map 6.16.1. To prove6.16 we prove the stronger statement that 8.19.2 is an isomorphism and in addition we will show that under this isomorphism the usual trace map on étale cohomology agrees with the one defined in 6.14 .

8.20. Let $\left(Y, D_{Y}\right)$ be another smooth proper $V$-scheme with $D_{Y}$ a divisor with simple normal crossings. Let $D_{Y}=E_{Y} \cup F_{Y}$ be a decomposition of the divisor into two divisors with no common irreducible component, and let $f: Y \rightarrow X$ be a morphism such that $f^{-1}(E) \subset E_{Y}$ and $f^{-1}(F) \subset F_{Y}$. It follows from the construction that then there is an induced morphism

$$
f^{*}: R \Gamma\left(\mathscr{X}_{\frac{o}{K}}, \mathscr{L} \otimes \mathscr{J}_{E}\right) \rightarrow R \Gamma\left(\mathscr{Y}_{\bar{K}}^{o}, f^{*} \mathscr{L} \otimes \mathscr{J}_{E_{Y}}\right) .
$$

and this morphism is compatible with cup products and the trace maps.

Let $d_{X}\left(\right.$ resp. $\left.d_{Y}\right)$ denote the relative dimension of $X($ resp. $Y$ ) over $V$. The map 8.20.1 induces a morphism

$$
\begin{gathered}
R \operatorname{Hom}\left(R \Gamma\left(\mathscr{Y}_{\bar{K}}^{o}, f^{*} \mathscr{L} \otimes \mathscr{J}_{E_{Y}}\right), \bar{V} / p^{r} \bar{V}\left(-d_{Y}\right)\left[-2 d_{Y}\right]\right) \\
\qquad \\
R \operatorname{Hom}\left(R \Gamma\left(\mathscr{X}_{\bar{K}}^{o}, \mathscr{L} \otimes \mathscr{J}_{E}\right), \bar{V} / p^{r} \bar{V}\left(-d_{X}\right)\left[-2 d_{X}\right]\right)\left(d_{X}-d_{Y}\right)\left[2\left(d_{X}-d_{Y}\right)\right] .
\end{gathered}
$$

Using 6.17.1 this morphism corresponds to a morphism

$$
f_{*}: R \Gamma\left(\mathscr{Y}_{\bar{K}}^{o}, f^{*} \mathscr{L}^{*} \otimes \mathscr{J}_{F_{Y}}\right) \rightarrow R \Gamma\left(\mathscr{X}_{K}^{o}, \mathscr{L}^{*} \otimes \mathscr{J}_{F}\right) \text {. }
$$

Similarly Poincaré duality for étale cohomology with partial compact supports 8.13 defines a morphism

$$
f_{*}^{\text {et }}: R \Gamma_{E_{Y}, F_{Y}}\left(Y_{\bar{K}}^{o}, f^{*} L\right) \rightarrow R \Gamma_{E, F}\left(X_{\bar{K}}^{o}, L\right)\left(d_{X}-d_{Y}\right)\left[2\left(d_{X}-d_{Y}\right)\right] .
$$

THEOREM 8.21. Assume $f$ is a closed immersion, and that $Y$ meets $D$ transversally, and $E_{Y}=E \cap Y$ and $F_{Y}=F \cap Y$. Then the diagram

$$
\begin{array}{cc}
R \Gamma\left(\mathscr{Y} \frac{o}{K}, f^{*} \mathscr{L} \otimes \mathscr{J}_{E_{Y}}\right) \stackrel{f_{*}}{\longrightarrow} & R \Gamma\left(\mathscr{X}_{\frac{o}{K}}^{o}, \mathscr{L} \otimes \mathscr{J}_{E}\right)\left(d_{X}-d_{Y}\right)\left[2\left(d_{X}-d_{Y}\right)\right] \\
\sigma_{Y} \uparrow & \uparrow \sigma_{X}
\end{array}
$$

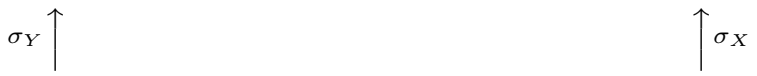

commutes in $\bar{V}^{a}$-Mod, where the vertical arrows are the morphisms 8.19.2.

Proof. Let us first consider the case when $Y \subset X$ is a smooth divisor meeting $D$ transversally (the general case will then be deduced from this special case). Let $Y^{o}$ denote $Y-(Y \cap D)$ and let $D_{Y}=Y \cap D=E_{Y} \cup F_{Y}$ be the decomposition of the divisor $D_{Y}$ obtained from $D=E \cup F$. Write $d$ for the relative dimension of $X$ over $V$, and let $\widetilde{X}^{o}$ denote $X^{o}-Y^{o}$. Let $\widetilde{\mathscr{X}_{\bar{K}}^{o}}$ denote the topos associated to the pair $(X, Y \cup D)$. Let $\widetilde{E}=E$ and $\widetilde{F}=F \cup Y$ so that we obtain a decomposition $Y \cup D=\widetilde{E} \cup \widetilde{F}$. The topos $\widetilde{\mathscr{X}} \frac{o}{K}$ is the category of sheaves on the site $\widetilde{\mathscr{S}}$ whose objects are pairs $(U, N)$ where $U \rightarrow X$ is étale and $N \rightarrow \widetilde{X}_{\bar{K}}^{o} \times_{X} U$ is finite étale and surjective. There is a natural functor

$$
\mathscr{S} \rightarrow \widetilde{\mathscr{S}}, \quad(U, N) \mapsto\left(U, N \times_{X^{\circ}} \tilde{X}^{o}\right)
$$


which induces a morphism of topoi $j: \widetilde{\mathscr{X}_{\bar{K}}^{o}} \rightarrow \mathscr{X}_{\bar{K}}^{o}$. There is also a morphism of topoi $i: \mathscr{Y}_{\bar{K}} \rightarrow \mathscr{X}_{\bar{K}}^{o}$. If $\mathscr{S}_{Y}$ denotes the site in the definition of $\mathscr{Y}_{\bar{K}}^{o}$ then the morphism $i$ is obtained from the functor

$$
\mathscr{S} \rightarrow \mathscr{S}_{Y}, \quad(U, N) \mapsto\left(U \times_{X} Y, N \times_{X} Y\right) .
$$

Proposition 8.22. Let $\mathscr{L}$ be as in 8.21. There is a canonical distinguished triangle

(8.22.1) $\mathscr{L} \otimes \mathscr{J}_{E} \rightarrow R j_{*}\left(j^{*} \mathscr{L} \otimes \mathscr{J}_{\widetilde{E}}\right) \rightarrow i_{*}\left(i^{*} \mathscr{L} \otimes \mathscr{J}_{E_{Y}}\right)(-1)[-1] \rightarrow \mathscr{L} \otimes \mathscr{J}_{E}[1]$ in $\widetilde{D}\left(\mathscr{X}_{\bar{K}}^{o}\right)$.

Proof. By the projection formula it suffices to consider the case when $\mathscr{L}=$ $\mathbb{Z} / p^{r}$ for some $r$. Let $(U, W) \in \mathscr{S}$ be an object. Then the localized topos $\left.\widetilde{\mathscr{X}_{\bar{K}}}\right|_{j^{-1}(U, W)}$ is isomorphic to the topos of sheaves on the site whose objects are pairs $\left(U^{\prime}, W^{\prime}\right)$, where $U^{\prime} \rightarrow U$ is étale and $W^{\prime} \rightarrow W \times_{X^{\circ}} \widetilde{X}^{o}$ is a finite étale morphism. Let $\widetilde{W}$ denote $W \times_{X^{o}} \widetilde{X}^{o}$. Any finite étale surjective morphism $W^{\prime} \rightarrow \widetilde{W}$ defines such a pair (namely $\left(U, W^{\prime}\right)$ ) and hence we obtain a morphism of topoi

$$
\epsilon:\left.\widetilde{\mathscr{X}}_{\bar{K}}^{o}\right|_{j-1}(U, W) \rightarrow \widetilde{W}_{\mathrm{Fet}} \text {. }
$$

If $U$ admits an étale morphism

$$
\pi: U \rightarrow \operatorname{Spec}\left(V\left[X_{1}, \ldots, X_{d+l}\right]\right) \times \operatorname{Spec}(V[y])
$$

for some integers $d, l \geq 0$, such that $Y$ is the inverse image of $\{y=0\}$ and $D$ is the inverse image of $\left\{X_{1} \cdots X_{d}=0\right\}$ (and say $E$ is the inverse image of $\left\{X_{1} \cdots X_{s}=\right.$ $0\})$ and $W=\operatorname{Spec}(R)$ for some $R$, then using the almost purity theorem we can compute

$$
H^{*}\left(\left.\widetilde{\mathscr{X}_{\bar{K}}^{o}}\right|_{j^{-1}(U, W)}, \mathscr{J}_{E} / p^{r} \mathscr{J}_{E}\right)
$$

as follows. Namely, let $\widetilde{S}_{\infty}$ denote the ring

$$
\widetilde{S}_{\infty}:=V\left[X_{1}^{1 / p^{\infty}}, \ldots, X_{d}^{1 / p^{\infty}}, y^{1 / p^{\infty}}\right]
$$

and let

$$
S_{\infty}:=V\left[X_{1}^{1 / p^{\infty}}, \ldots, X_{d}^{1 / p^{\infty}}, y\right] .
$$

Then if $\bar{J}_{\widetilde{E}} \subset \widetilde{S}_{\infty}$ denotes the ideal $\left(X_{1} \cdots X_{s}\right)$ we have as in the proof of 6.13 almost isomorphisms

$$
\begin{aligned}
H^{*}\left(\left.\widetilde{\mathscr{X}}_{\bar{K}}^{o}\right|_{j-1}(U, W), \mathscr{J}_{\widetilde{E}} / p^{r} \mathscr{J}_{\widetilde{E}}\right) & \simeq H^{*}\left(\widetilde{W}_{\mathrm{Fet}}, \epsilon_{*} \mathscr{J}_{\widetilde{E}} / p^{r} \mathscr{J}_{\widetilde{E}}\right) \\
& \simeq R \otimes_{S} H^{*}\left(\widetilde{\Delta}_{\infty}, \bar{J}_{\widetilde{E}} / p^{r} \bar{J}_{\widetilde{E}}\right),
\end{aligned}
$$

where $\widetilde{\Delta}_{\infty}$ denotes the Galois group of $\widetilde{S}_{\infty}$ over $S$. Let $\Delta_{\infty}$ denote the Galois group of $S_{\infty}$ over $S$ so that there is a surjection $\widetilde{\Delta}_{\infty} \rightarrow \Delta_{\infty}$ with kernel $Q$ isomorphic to $\mathbb{Z}_{p}(1)$. The kernel $Q$ corresponds to the étale covers of $\widetilde{W}$ obtained by taking roots of $y$. We then have the Hochschild-Serre spectral sequence

$$
E_{2}^{i j}=H^{i}\left(\Delta_{\infty}, H^{j}\left(Q, \bar{J}_{\widetilde{E}} / p^{r} \bar{J}_{\widetilde{E}}\right)\right) \Longrightarrow H^{i+j}\left(\widetilde{\Delta}_{\infty}, \bar{J}_{\widetilde{E}} / p^{r} \bar{J}_{\widetilde{E}}\right) .
$$

Now for any cohomology class $c \in H^{i}\left(\Delta_{\infty}, H^{j}\left(Q, \bar{J}_{\widetilde{E}} / p^{r} \bar{J}_{\widetilde{E}}\right)\right)$ with $i>0$, there exists a finite index subgroup $\Delta_{\infty}^{\prime} \subset \Delta_{\infty}$, corresponding to a covering $W^{\prime} \rightarrow$ $W$ obtained by extracting some roots of the $X_{i}$, such that $c$ maps to zero in 
$H^{i}\left(\Delta_{\infty}^{\prime}, H^{j}\left(Q, \bar{J}_{\widetilde{E}} / p^{r} \bar{J}_{\widetilde{E}}\right)\right)$. It follows that the sheaf on $W_{\text {Fet }}$ associated to the presheaf sending a finite étale morphism $W^{\prime} \rightarrow W$ to

$$
H^{i}\left(\left.\widetilde{\mathscr{X}_{\bar{K}}^{o}}\right|_{j-1}\left(U, W^{\prime}\right), \mathscr{J}_{\widetilde{E}} / p^{r} \mathscr{J}_{\widetilde{E}}\right)
$$

is almost isomorphic to the sheaf corresponding to the $\Delta_{\infty}$-representation

$$
H^{i}\left(Q, \bar{J}_{\widetilde{E}} /\left(p^{r}\right)\right) \otimes_{S} R
$$

We conclude that the sheaf on $\mathscr{S}$ associated to the presheaf

$$
(U, W) \mapsto H^{i}\left(\left.\widetilde{\mathscr{X}_{\bar{K}}^{o}}\right|_{j-1}\left(U, W^{\prime}\right), \mathscr{J}_{\widetilde{E}} / p^{r} \mathscr{J}_{\widetilde{E}}\right)
$$

is almost isomorphic to the sheaf associated to the presheaf whose restriction to $W_{\text {Fet }}$ for any object $(U, W)$ as above is the sheaf corresponding to $H^{i}\left(Q, \bar{J}_{\widetilde{E}} /\left(p^{r}\right)\right) \otimes_{S}$ $R$.

Note that as a $Q$-module, $\bar{J}_{\widetilde{E}}$ is isomorphic to $\bar{J}_{E} \otimes \bar{V}\left[y^{1 / p^{\infty}}\right]$. Thus we have

$$
H^{i}\left(Q, \bar{J}_{\widetilde{E}} / p^{r} \bar{J}_{\widetilde{E}}\right) \otimes_{S} R \simeq H^{i}\left(Q, \bar{V}\left[y^{1 / p^{\infty}}\right] /\left(p^{r}\right)\right) \otimes_{V[y]} J_{E} \otimes_{S} R .
$$

This reduces us to the case when $R=V[y]$. We may further localize along $1+y$. So we consider the ring $R=V\left[\kappa^{ \pm}\right]$with divisor defined by $y:=\kappa-1$, and even pass to the covering obtained by taking roots of $\kappa$ (which gives an étale covering after inverting $p$ ). Let $V_{m}$ denote $V\left[\pi^{1 / m}\right]$, and let

$$
R_{m}:=V_{m}\left[\kappa^{ \pm 1 / p^{n}}\right]^{\text {sh }} \text {, }
$$

where the superscript 'sh' denotes strict henselization at the point defined by $\left(\pi^{1 / m}, \kappa^{1 / p^{n}}-1\right)$. Let $R_{\infty}$ denote $\lim _{m} R_{m}$, which is a $\bar{V}$-algebra. Also let $S_{n, m}$ denote the normalization of the ring $R_{m}\left[y^{1 / p^{n}}\right]$, and let $S_{n}=\lim _{m} S_{n, m}$. Finally set $S_{\infty}=\lim _{\longrightarrow} S_{n}$. Let $J_{n, m} \subset S_{n, m}$ (resp. $J_{n} \subset S_{n}$ ) be the ideal generated by $y^{1 / p^{n}}$.

We then need to compute the cohomology groups $H^{i}\left(\mathbb{Z}_{p}(1), S_{\infty} / p^{r} S_{\infty}\right)$. This is a rather delicate computation which is explained in detail in [13. pp. 231-233]. The answer is the following (almost isomorphisms):

(i) $H^{i}\left(\mathbb{Z}_{p}(1), S_{\infty} / p^{r} S_{\infty}\right) \simeq R_{\infty} / p^{s} R_{\infty}$;

(ii) $H^{1}\left(\mathbb{Z}_{p}(1), S_{\infty} / p^{r} S_{\infty}\right)=\left(R_{\infty} / y R_{\infty}\right)^{\text {norm }} \otimes\left(\mathbb{Z} / p^{r}\right)(-1)$, where $\left(R_{\infty} / y R_{\infty}\right)^{\text {norm }}$ denotes the normalization of $R_{\infty} / y R_{\infty}$.

(iii) $H^{i}\left(\mathbb{Z}_{p}(1), S_{\infty} / p^{r} S_{\infty}\right)=0$ for $i>1$.

This implies that $R^{1} j_{*} j^{*} \mathscr{J}_{\widetilde{E}} / p^{r} \mathscr{J}_{\widetilde{E}}$ is annihilated by $y$ and there is a canonical almost epimorphism

$$
i_{*}\left(\mathscr{J}_{E_{Y}} / p^{r} \mathscr{J}_{E_{Y}}\right) \rightarrow R^{1} j_{*} j^{*} \mathscr{J}_{\widetilde{E}} / p^{r} \mathscr{J}_{\widetilde{E}} .
$$

That this morphism is in fact an almost isomorphism follows by reduction to the case of $\mathbb{A}^{1}$ via the same argument used above in which case it follows from (ii).

Similarly on $(X-F)_{\bar{K}}$ there is a distinguished triangle of étale sheaves

$$
j_{E !} L \rightarrow R j_{*} j^{*} j_{E !} L \rightarrow i_{*} i^{*} j_{E !} L(-1)[-1] \rightarrow j_{E !} L[1],
$$

where $j_{E}: X-D \hookrightarrow X-F$ and $j: X-Y \cup F \hookrightarrow X-F$ are the inclusions. Indeed using 8.14 it suffices to consider the higher direct images of $j^{*} L$ under $j: X-Y \cup D \rightarrow X-D$. Furthermore, by the projection formula it suffices to consider the case of $L=\mathbb{Z} /\left(p^{r}\right)$. By working locally on $X$ one further reduces to computing $R^{1} j_{*} \mathbb{Z} /\left(p^{r}\right)$ for the inclusion of $\mathbb{G}_{m} \hookrightarrow \mathbb{A}^{1}$ which follows from a group cohomology computation as in 3.5 . 
Proposition 8.23. The morphisms denoted momentarily by $i_{*}^{\prime}$ and $i_{*}^{\text {et }}$

$$
i_{*}^{\prime}: R \Gamma\left(\mathscr{Y}_{\bar{K}}^{o}, i^{*} \mathscr{L} \otimes \mathscr{J}_{\widetilde{E}}\right) \rightarrow R \Gamma\left(\mathscr{X}_{\bar{K}}^{o}, \mathscr{L} \otimes \mathscr{J}_{E}\right)(1)[2]
$$

and

$$
i_{*}^{\text {et }}: R \Gamma_{\widetilde{E}, \widetilde{F}}\left(Y_{\bar{K}}^{o}, i^{*} L\right) \rightarrow R \Gamma_{E, F}\left(X_{\bar{K}}^{o}, L\right)(1)[2]
$$

obtained from the boundaries in the long exact sequences obtained from 8.22.1 and 8.22.11 agree with the maps $i_{*}$ and $i_{*}^{\text {et }}$ respectively.

Proof. Note first of all that the diagrams

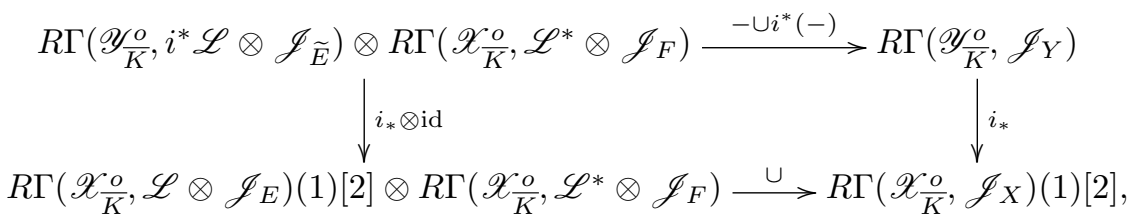

and

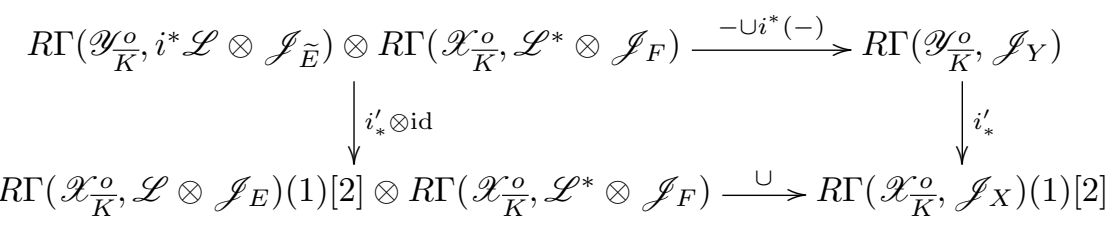

commute. It follows that it suffices to consider the case when $L=\mathbb{Z} /\left(p^{r}\right)$, and $E=D($ so $F=\emptyset)$. Furthermore, by Poincaré duality it suffices to show that the boundary maps

$$
i_{*}^{\prime}: H^{2(d-1)}\left(\mathscr{Y}_{\bar{K}}^{o}, i^{*} \mathscr{L} \otimes \mathscr{J}_{Y}\right) \rightarrow H^{2 d}\left(\mathscr{X}_{\bar{K}}^{o}, \mathscr{L} \otimes \mathscr{J}_{X}\right)
$$

and

$$
i_{*}^{\text {et }}: H_{c}^{2(d-1)}\left(Y_{\bar{K}}^{o}, i^{*} L\right) \rightarrow H_{c}^{2 d}\left(X_{\bar{K}}^{o}, L\right)
$$

are compatible with the trace maps.

This is well-known in the case of étale cohomology. Indeed by the construction the induced map

$$
H_{c}^{*}\left(Y_{\bar{K}}^{o}, R i^{!} \mathbb{Z} / p^{r}\right) \simeq H_{c}^{*}\left(Y_{\bar{K}}^{o}, \mathbb{Z} / p^{r}(-1)[-2]\right) \rightarrow H_{c}^{*}\left(X_{\bar{K}}^{o}, \mathbb{Z} / p^{r}\right)
$$

given by the boundary of 8.22 .1 is equal to the map induced by the adjunction $R i_{1} R i^{!} \mathbb{Z} / p^{r} \rightarrow \mathbb{Z} / p^{r}$. This combined with construction of the trace map in 9 , Cycle, 2.3] shows that the two trace maps in the étale theory coincide.

For the compatibility of $i_{*}^{\prime}$ with the trace maps it suffices to study the sequence

$$
\cdots R^{d} \nu_{X *} \mathscr{J}_{X} \rightarrow R j_{*} j^{*} R^{d} \nu_{X *} \mathscr{J}_{X} \rightarrow R^{d-1} \nu_{X *} i_{*} \mathscr{J}_{Y} \rightarrow 0 .
$$

obtained by applying $R \nu_{X *}$ to the sequence 8.22.1. Now a local calculation as in the proof of 4.7 shows that this sequence is almost isomorphic to the sequence

$$
\cdots \Omega_{X}^{d} \rightarrow \Omega_{X}^{d}(\log Y) \rightarrow \Omega_{Y}^{d-1} \rightarrow 0 .
$$

Here we have used the isomorphism between $\Omega_{Y}^{d-1}$ and

$$
\operatorname{Coker}\left(\Omega_{X}^{d} \rightarrow \Omega_{X}^{d}(\log Y)\right) \simeq i^{*} \Omega_{X}^{d}(\log Y)
$$


obtained by choosing locally a local coordinate $y$ for $Y$ in $X$ and defining the isomorphism

$$
\Omega_{Y}^{d-1} \rightarrow i^{*} \Omega_{X}^{d}(\log Y), \quad \omega \mapsto i^{*}(\tilde{\omega} \wedge d \log (y)),
$$

where $\tilde{\omega} \in \Omega_{X}^{d-1}$ is a lifting of $\omega$. If $u \in \mathscr{O}_{X}^{*}$ is a unit, then $d \log (u y)$ is equal to $u^{-1} d u+d \log (y)$ from which one sees that the map 8.23 .7 is independent of the choices. We thus get a global isomorphism. It follows that $i_{*}^{\prime}$ is induced from the boundary map

$$
H^{d-1}\left(Y, \Omega_{Y}^{d-1}\right) \rightarrow H^{d}\left(X, \Omega_{X}^{d}\right)
$$

obtained from the short exact sequence

$$
0 \rightarrow \Omega_{X}^{d} \rightarrow \Omega_{X}^{d}(\log Y) \rightarrow i_{*} \Omega_{Y}^{d-1} \rightarrow 0 .
$$

We leave to the reader the task of verifying that this map is equal under Serre duality to the canonical isomorphism

$$
H^{0}\left(X, \mathscr{O}_{X}\right) \simeq H^{0}\left(Y, \mathscr{O}_{Y}\right)
$$

thereby completing the proof.

8.24. We obtain a diagram

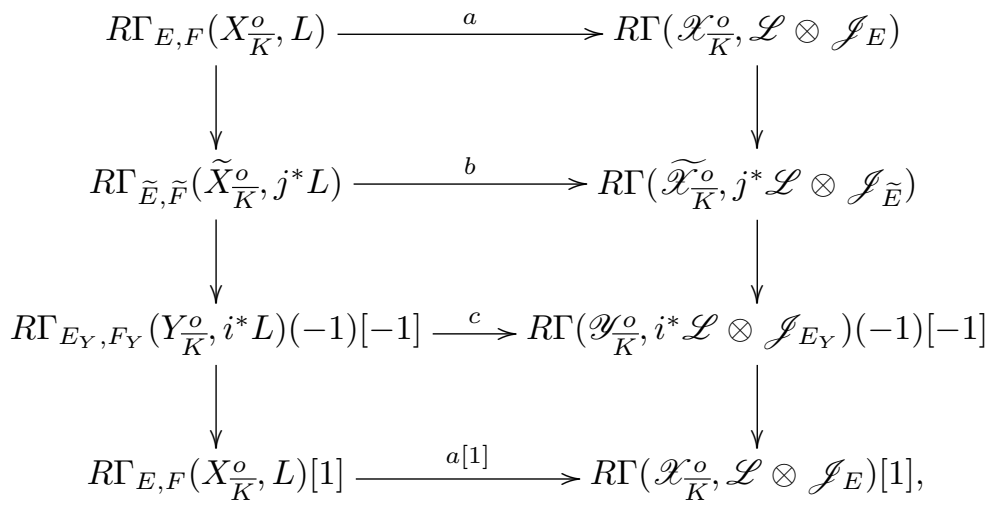

where the columns are the distinguished triangles obtained by applying the global section functor to 8.22.1 and 8.22.11, and the horizontal arrows are the morphisms 8.19 To prove 8.21 in the present special case it suffices to show that 8.24.1 commutes.

8.25. First let us make some general observations about the construction of the complexes $\Psi(-), \bar{\Psi}(-)$, and $\Phi(-)$.

Let $\widetilde{\Delta}^{+}$denote the category whose objects are finite ordered sets (including the empty set!) and whose morphisms are order preserving monomorphisms. Let $\underline{\Delta}^{+} \subset \widetilde{\Delta}^{+}$denote the full subcategory of nonempty ordered sets. For $n \geq 0$ let $[n]$ denote the ordered set $\{0,1, \ldots, n\}$ and by convention we define $[-1]$ to be the empty set.

For a topos $T$ let $T \widetilde{\underline{\Delta}}^{+}$denote the constant $\widetilde{\widetilde{\Delta}}^{+}$-topos associated to $T$ (see for example $\left[1, \mathrm{~V}^{\mathrm{bis}}\right]$ for the notion of a $D$-topos for a category $D$ ). So a sheaf $F \in T \widetilde{\widetilde{\Delta}}^{+}$ consists of a sheaf $F_{n}$ for every $[n] \in \widetilde{\widetilde{\Delta}}^{+}$and for every inclusion $[n] \hookrightarrow[m]$ a morphism $F_{n} \rightarrow F_{m}$. In other words, the restriction of $F$ to $\underline{\Delta}^{+}$is a strictly 
cosimplicial sheaf in $T$ and so the category $T^{\widetilde{\Xi}^{+}}$is equivalent to the category of triples $\left(F_{-1}, F_{+}, \sigma\right)$, where $F_{-1}$ is in $T, F_{+}$is a strictly cosimplicial sheaf in $T$, and $\sigma: F_{-1} \rightarrow F_{+}$is an augmentation (where $F_{-1}$ is viewed as a constant cosimplicial sheaf). If $S$ is a site defining $T$, then the topos $T \widetilde{\underline{\Delta}}^{+}$is isomorphic to the topos associated to the site $S \underline{\underline{\Delta}}^{+}$whose objects are pairs $(U,[n])$, where $U \in S$ and $[n] \in \widetilde{\widetilde{\Delta}}^{+}$, and whose morphisms $\left(U^{\prime},\left[n^{\prime}\right]\right) \rightarrow(U,[n])$ are pairs $f: U^{\prime} \rightarrow U$ and $\delta:[n] \hookrightarrow\left[n^{\prime}\right]$, where $f$ is a morphism in $S$ and $\delta$ is a morphism in $\underline{\widetilde{\Delta}}^{+}$. Such a morphism is a covering if $f$ is a covering in $S$ and $[n]=\left[n^{\prime}\right]$.

8.26. If $\Lambda$ is a ring in $T$, then $T \widetilde{\widetilde{\Delta}}^{+}$is also ringed by the sheaf of rings which in degree $n$ is equal to $\Lambda$. Let $D^{+}\left(T \widetilde{\widetilde{\Delta}}^{+}, \Lambda\right)$ denote the bounded below derived category of complexes of $\Lambda$-modules in $T \widetilde{\underline{\Delta}}^{+}$. There is a total complex functor

$$
\int: D^{+}\left(T^{\widetilde{\Delta}^{+}}, \Lambda\right) \rightarrow D^{+}(T, \Lambda)
$$

defined as follows. A $\Lambda$-module $K \in T \widetilde{\underline{\Delta}}^{+}$defines a complex in $T$ whose degree $r$-term is $K([r-1])$ and whose transition morphism $K([r-1]) \rightarrow K([r])$ is given by the sum

$$
\sum_{i=0}^{r}(-1)^{i} \partial_{i}: K([r-1]) \rightarrow K([r]),
$$

where $\partial_{i}:[r-1] \rightarrow[r]$ is the unique injective map whose image does not contain $i$ (if $r=0$ we take the map induced by the unique map $[-1] \rightarrow[0]$ ). This construction is functorial in $K$ and therefore if $K^{\bullet}$ is a complex of $\Lambda$-modules in $T^{\widetilde{\Omega}^{+}}$we obtain a bicomplex by applying the above construction in each degree. We define $\int K^{\bullet}$ to be the associated single complex. We also sometimes refer to this complex as the normalized complex of $K^{\bullet}$.

8.27. A complex $K^{\bullet}$ in $T \widetilde{\underline{\Delta}}^{+}$is equivalent to a complex $K_{-1}^{\bullet}$ in $T$, a strictly cosimplicial complex $K_{+}^{\bullet}$ in $T$, and an augmentation $K_{-1}^{\bullet} \rightarrow K_{+}^{\bullet}$. If $\operatorname{Tot}\left(K_{+}^{\bullet}\right)$ denotes the usual total complex of the cosimplicial complex $K_{+}^{\bullet}$, then the augmentation $K_{-1}^{\bullet} \rightarrow K_{+}^{\bullet}$ induces a morphism of complexes $K_{-1}^{\bullet} \rightarrow \operatorname{Tot}\left(K_{+}^{\bullet}\right)$ and $\int K_{\bullet}$ is the cone of this morphism of complexes. It follows that $\int$ takes quasi-isomorphisms to quasi-isomorphisms and hence passes to the derived category. Moreover this implies that $\int$ takes distinguished triangles to distinguished triangles. This defines the triangulated functor 8.26 .1 .

For a morphism $f: T^{\prime} \rightarrow T$ of topoi there is an induced morphism of topoi $T^{\prime \widetilde{\underline{\Delta}}^{+}} \rightarrow T \widetilde{\underline{\underline{\Delta}}}^{+}$which we usually denote by the same letter $f$. If $T^{\prime}$ and $T$ are ringed by $\Lambda^{\prime}$ and $\Lambda$ respectively, and $f$ is a morphism of ringed topoi then the diagram

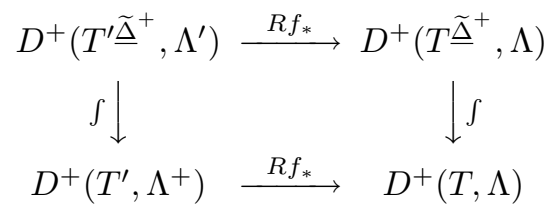

commutes.

We use this to prove that 8.24 .1 commutes as follows. To ease the notation, we give the proof in the case when $E=D$ so that $F=\emptyset$. The general case is obtained by exactly the same argument replacing $X$ in the following by $X-F$. 
Let $s: X^{o} \hookrightarrow X$ be the inclusion, and write also $s$ for the inclusions $Y^{o} \hookrightarrow Y$, $\widetilde{X}^{o} \hookrightarrow \tilde{X}, X_{\bar{K}}^{o} \hookrightarrow X_{\bar{K}}$ etc.

Lemma 8.28. Let $U \rightarrow X$ be an étale morphism and let $L$ be a sheaf of $\Lambda_{-}^{-}$ modules on $U_{\bar{K}, \mathrm{Fet}}^{o}$. Set $Y_{U, \bar{K}}:=U_{\bar{K}} \times_{X} Y$ so that we have a cartesian diagram

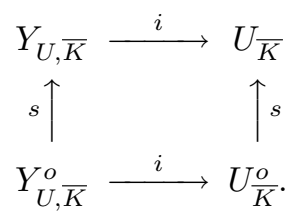

Then the natural map in the derived category of $\Lambda$-modules in $Y_{U, \bar{K} \text {,et }}$

$$
i^{*} s_{*} L \rightarrow s_{*} i^{*} L
$$

is an isomorphism.

Proof. Let $\bar{y} \rightarrow Y_{U, \bar{K}}$ be a geometric point. Base changing the diagram 8.28.1 to $\operatorname{Spec}\left(\mathscr{O}_{U_{\bar{K}}, \bar{y}}\right)$ we reduce the proof to the analogous statement for $\operatorname{Spec}\left(\mathscr{O}_{U_{\bar{K}}, \bar{y}}\right)$. In this case the result is immediate because $U_{\bar{K}}^{o}$ and $Y_{U, \bar{K}}^{o}$ are $K(\pi, 1)$ 's and the map $Y_{U, \bar{K}}^{o} \rightarrow U_{\bar{K}}^{o}$ induces an isomorphism on fundamental groups.

8.29. Let $U . \in H R(X)^{\prime}$ be a hypercover of $X$, where $H R(X)^{\prime}$ is the category defined in 9.4 Let $\widetilde{U}_{\cdot, \bar{K}}$ denote $U_{\cdot, \bar{K}} \times_{X} \widetilde{X}$, and let $Y_{U, \cdot, \bar{K}}$ denote $U_{\cdot, \bar{K}} \times_{X} Y$. We then have a commutative diagram of simplicial topoi

$$
\widetilde{U}_{\cdot, \bar{K}, \text { et }} \stackrel{j}{\longrightarrow} U_{\cdot, \bar{K}, \text { et }} \stackrel{i}{\longleftarrow} Y_{U, \cdot, \bar{K}, \text { et }} .
$$

For a sheaf $F$. in $U_{,{ }^{\prime}, \bar{K}, \text { Fet }}^{o}$ we then obtain as in 7.21 complexes $\bar{\Psi}\left(j^{*} F\right), \bar{\Psi}(F)$, and $\bar{\Psi}\left(i^{*} F\right)$ on $\widetilde{U}_{\cdot, \bar{K}, \text { et }}, U_{\cdot, \bar{K}, \text { et }}$, and $Y_{U, \cdot, \bar{K} \text {,et }}$ respectively. It follows immediately from the construction of these complexes that $\bar{\Psi}\left(j^{*} F\right)$ is canonically isomorphic to $j^{*} \bar{\Psi}(F)$. Also the maps 8.28 .2 define a morphism

$$
i^{*} \bar{\Psi}(F) \rightarrow \bar{\Psi}\left(i^{*} F\right)
$$

which by 8.28 is an isomorphism.

The diagram 8.29.1 extends to a diagram of $\underline{\widetilde{\Delta}}^{+}$-topoi

$$
\widetilde{U}_{\cdot, \bar{K}, \text { et }}^{\widetilde{\widetilde{\Delta}}^{+}} \stackrel{j}{\longrightarrow} U_{\cdot, \bar{K}, \text { et }}^{\widetilde{\widetilde{\Delta}}^{+}} \stackrel{i}{\longleftarrow} Y_{U, \cdot, \bar{K}, \text { et }}^{\widetilde{\widetilde{\Delta}}^{+}} .
$$

Define $\bar{\Psi}_{\widetilde{\Delta}^{+}}(F)$ to be the sheaf in $U_{\cdot, \bar{K}, \text { et }} \widetilde{\widetilde{\Delta}}^{+}$whose restriction to $U_{r, \bar{K} \text {,et }}($ for $r \geq-1$ ) is $\bar{\Psi}(F)^{r+1}$ and whose transition maps are given by the morphisms 7.21 .2 . Then $\bar{\Psi}(F)$ is equal to $\int \bar{\Psi}_{\widetilde{\underline{\Delta}}^{+}}(F)$. Similarly we have sheaves $\bar{\Psi}_{\widetilde{\Delta}^{+}}\left(j^{*} F\right)$ and $\bar{\Psi}_{\widetilde{\underline{\Delta}}^{+}}\left(i^{*} F\right)$ on $\widetilde{U}_{,, \bar{K}, \text { et }}^{\widetilde{\widetilde{\Delta}}^{+}}$and $Y_{U, \cdot, \bar{K}, \text { et }}^{\widetilde{\widetilde{\Delta}}^{+}}$respectively. The isomorphism 8.29 .2 is then induced by an isomorphism $i^{*} \bar{\Psi}_{\widetilde{\underline{\Delta}}^{+}}(F) \rightarrow \bar{\Psi}_{\widetilde{\underline{\Delta}}^{+}}\left(i^{*} F\right)$.

Lemma 8.30. Assume $F$ is an injective sheaf in $U_{,, \bar{K}, \mathrm{Fet}}^{o}$. Then the natural map of sheaves in $U_{\cdot, \bar{K}, \text { et }}^{\widetilde{\underline{\Delta}}^{+}}$

$$
\bar{\Psi}_{\widetilde{\underline{\Delta}}^{+}}(F) \otimes^{\mathbb{L}} R j_{*} \Lambda \rightarrow R j_{*} j^{*} \bar{\Psi}_{\underline{\underline{\widetilde{D}}}^{+}}(F)
$$

is an isomorphism. 
Proof. It suffices to verify that 8.30 .1 induces an isomorphism on stalks at a geometric point $\bar{y} \rightarrow U_{n, \bar{K}}$. Let $W$ denote $\operatorname{Spec}\left(\mathscr{O}_{U_{n, \bar{K}}, \bar{y}}\right)$, let $Y_{W} \subset W$ denote the inverse image of $Y$, let $\widetilde{W}$ denote the inverse image of $\widetilde{U}$, and define $W_{A}^{o}$ etc. as before. Let $\Delta$ denote the fundamental group of $W^{o}$, and let $\widetilde{\Delta}$ denote the fundamental group of $\widetilde{W}^{o}$. The fundamental group of $W^{o}$ (resp. $\widetilde{W}^{o}$ ) is isomorphic to $\widehat{\mathbb{Z}}(1)^{d}$ (resp. $\widehat{\mathbb{Z}}(1)^{d+1}$ ) for some integer $d$, and the map from $\Delta$ (resp. $\widetilde{\Delta}$ ) to the fundamental group of $U_{n, \bar{K}}^{o}$ (resp. $\widetilde{U}_{n, \bar{K}}^{o}$ ) is injective since we have by assumption an étale morphism 7.1.1. It follows from 7.18 that the pullback of $F$ to $W^{o}$ is an injective $\Delta$-representation, so it suffices to prove the lemma for $W$.

Let $M$ be the representation corresponding to $F$. Then $\bar{\Psi}_{\widetilde{\Delta}^{+}}(F)$ is a direct sum of sheaves of the form $j_{A *} j_{A}^{*} j_{*} F$ for subsets $A \subset\{1, \ldots, m\}$. Let $I_{A} \subset \Delta$ (resp. $\widetilde{I}_{A} \subset \widetilde{\Delta}$ ) denote the inertia group of $D_{A}$. Then the right side of 8.30 .1 computes the direct sum of the groups

$$
H^{*}\left(\widetilde{\Delta} / \widetilde{I}_{A}, M^{I_{A}}\right) .
$$

On the other hand, the map $\widetilde{I}_{A} \rightarrow I_{A}$ is an isomorphism, so the restriction of $M$ to $\widetilde{I}_{A}$ is an injective representation. Therefore 8.30 .2 is isomorphic to

$$
H^{*}(\widetilde{\Delta}, M) \text {. }
$$

From the Leray spectral sequence associated to the map $\widetilde{\Delta} \rightarrow \Delta$ (whose kernel is isomorphic to $\widehat{\mathbb{Z}}(1))$ and the fact that $M$ is an injective $\Delta$-representation we find that

$$
H^{0}(\widetilde{\Delta}, M) \simeq M^{\Delta}, \quad H^{1}(\widetilde{\Delta}, M) \simeq H^{0}\left(\Delta, M \otimes H^{1}(\widehat{\mathbb{Z}}(1), \Lambda)\right),
$$

and all other cohomology groups are zero.

To compute the left side of 8.30 .1 note that the distinguished triangle

$$
\Lambda \rightarrow R j_{*} \Lambda \rightarrow i_{*} \Lambda(-1)[-1] \rightarrow \Lambda[1],
$$

where $i: W_{Y} \hookrightarrow W$ denotes the inclusion shows that we have

$$
H^{0}\left(W, \bar{\Psi}_{\widetilde{\underline{\Delta}}^{+}}(F) \otimes^{\mathbb{L}} R j_{*} \Lambda\right)=\oplus_{A \subset\{1, \ldots, m\}} M^{\Delta},
$$

and

$$
\begin{aligned}
& H^{i}\left(W, \bar{\Psi}_{\widetilde{\underline{\Omega}}^{+}}(F) \otimes^{\mathbb{L}} R j_{*} \Lambda\right)=H^{i-1}\left(W, \bar{\Psi}_{\widetilde{\underline{\underline{D}}}^{+}}(F) \otimes i_{*} \Lambda(-1)\right) \\
& \simeq H^{i-1}\left(W_{Y}, i^{*} \bar{\Psi}_{\underline{\underline{\Delta}}^{+}}(F)\right)(-1) \text {. }
\end{aligned}
$$

for $i>0$. Since $W_{Y}$ is strictly henselian local, we get that

$$
H^{i}\left(W, \bar{\Psi}_{\widetilde{\underline{\Delta}}^{+}}(F) \otimes \mathbb{L} R j_{*} \Lambda\right)=0
$$

for $i>1$, and that

$H^{1}\left(W, \bar{\Psi}_{\widetilde{\underline{\underline{ }}}^{+}}(F) \otimes^{\mathbb{L}} R j_{*} \Lambda\right)=H^{0}\left(W_{Y}, i^{*} \bar{\Psi}_{\widetilde{\underline{\Delta}}^{+}}(F)\right)(-1)=\oplus_{A \subset\{1, \ldots, m\}} M^{\Delta}(-1)$.

8.31. Assume now that $F$ is an injective sheaf in $U_{\cdot, \bar{K}, \mathrm{Fet}}$. In $U_{\cdot, \bar{K}, \text { et }}^{\widetilde{\widetilde{\Delta}}^{+}}$we then obtain a distinguished triangle

$$
\bar{\Psi}_{\widetilde{\underline{\Delta}}^{+}}(F) \rightarrow R j_{*} \bar{\Psi}_{\widetilde{\underline{\Delta}}^{+}}\left(j^{*} F\right) \rightarrow i_{*} \bar{\Psi}_{\widetilde{\underline{\Delta}}^{+}}\left(i^{*} F\right)(-1)[-1] \rightarrow \bar{\Psi}_{\widetilde{\underline{\Delta}}^{+}}(F)[1]
$$


by tensoring the usual distinguished triangle

$$
\Lambda \rightarrow R j_{*} \Lambda \rightarrow i_{*} \Lambda(-1)[-1] \rightarrow \Lambda[1]
$$

with $\bar{\Psi}_{\widetilde{\Delta}^{+}}(F)$ and applying 8.30 . Applying the total complex functor we obtain a distinguished triangle

$$
\bar{\Psi}(F) \rightarrow R j_{*} \bar{\Psi}\left(j^{*} F\right) \rightarrow i_{*} \bar{\Psi}\left(i^{*} F\right)(-1)[-1] \rightarrow \bar{\Psi}(F)[1]
$$

in $U \cdot, \bar{K}$, et .

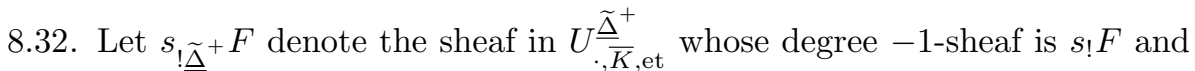
0 in all degrees $\geq 0$, and define $s_{! \underline{\underline{\Delta}}}\left(j^{*} F\right)$ and $s_{! \underline{\underline{\Delta}}}^{+}\left(i^{*} F\right)$ similarly. The maps

$$
s_{!} F \rightarrow \bar{\Psi}(F)^{0}, \quad s ! j^{*} F \rightarrow \bar{\Psi}\left(j^{*} F\right)^{0}, \quad s ! i^{*} F \rightarrow \bar{\Psi}\left(i^{*} F\right)^{0}
$$

induce morphisms of sheaves

$$
s_{! \underline{\underline{\Delta}}^{+}}(F) \rightarrow \bar{\Psi}_{\widetilde{\underline{\Delta}}^{+}}(F), \quad s_{! \underline{\tilde{\Delta}^{+}}} j^{*} F \rightarrow \bar{\Psi}_{\widetilde{\underline{\Delta}}^{+}}\left(j^{*} F\right), \quad s_{! \underline{\tilde{\Delta}^{+}}}\left(i^{*} F\right) \rightarrow \bar{\Psi}_{\underline{\underline{\underline{\Delta}}}^{+}}\left(i^{*} F\right) .
$$

These maps induce a diagram in the derived category of $\Lambda$-modules on $U_{\cdot, \bar{K}}^{\widetilde{\Delta}^{+} \text {et }}$

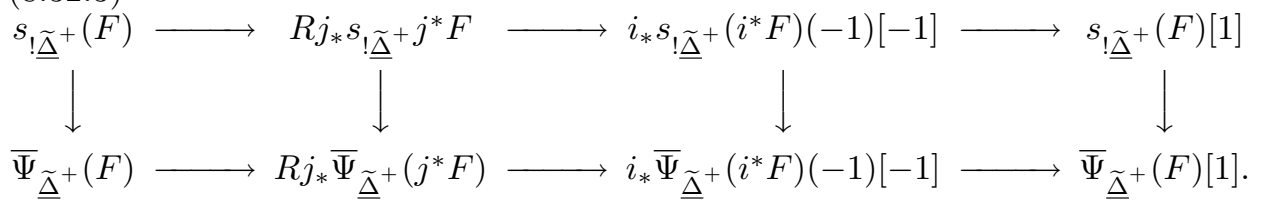

LEMMA 8.33. The diagram 8.32.3 commutes.

PROOF. This follows from the construction.

8.34. Similarly, we can consider the diagram of $\underline{\Delta}^{+}$-topoi

$$
\widetilde{\mathscr{X}}_{\bar{K}}^{o \widetilde{\Delta}^{+}} \stackrel{j}{\longrightarrow} \mathscr{X}_{\frac{o}{K}}^{o \widetilde{\Delta}^{+}} \longleftarrow i \quad \mathscr{Y}_{\bar{K}}^{o \underline{\Delta}^{+}}
$$

Let $G$ be a sheaf of $\Lambda$-modules in $\mathscr{X}_{\frac{o}{K}}$. The complexes $\Phi^{a}\left(\mathscr{O}_{\mathscr{X}_{\frac{o}{K}}}\right)$ and $\Psi^{a}(G)$ defined in 8.15 and 8.3 are then also obtained from $\widetilde{\underline{\Delta}}^{+}$-versions $\Phi_{\widetilde{\Delta}^{+}}^{a}\left(\mathscr{O}_{\mathscr{X}_{\frac{\mathrm{K}}{K}}}\right)$ and $\Psi_{\widetilde{\underline{\Delta}}^{+}}^{a}(G)$. Namely, let $\Phi_{\widetilde{\Delta}^{+}}^{a}\left(\mathscr{O}_{\mathscr{X}_{\frac{\mathrm{K}}{\mathrm{K}}}}\right)$ be the sheaf whose degree $r$-component $(r \geq-1)$ is equal to $\Phi^{a}\left(\mathscr{O}_{\mathscr{X}_{\frac{o}{K}}}\right)^{r+1}$ and whose transition maps are defined as in 8.15.4. Note that the sheaf $\Phi_{\widetilde{\underline{\Delta}}^{+}}^{a}\left(\mathscr{O}_{\mathscr{X}_{\frac{o}{K}}}\right)$ is actually a sheaf of rings. The sheaf $\Psi_{\widetilde{\underline{\Delta}}^{+}}^{a}(G)$ is the sheaf whose degree $r$ component is $\Psi^{a}(G)^{r+1}$ and again the transition maps are defined as in 7.26 .

Assume now further that every $(U, N) \in \mathscr{S}$ (object of the site defining $\mathscr{X}_{\bar{K}}$ ) the corresponding sheaf $G_{(U, N)}$ on $N_{\text {Fet }}$ is a locally constant constructible sheaf of flat $\Lambda$-modules. Then using the same argument that proved 8.22 , one sees that there is a distinguished triangle $(8.34 .2)$

$G \otimes \Phi_{\widetilde{\underline{\Delta}}^{+}}^{a}\left(\mathscr{O}_{\mathscr{X}_{\frac{o}{K}}}\right) \rightarrow R j_{*}\left(j^{*} G \otimes \Phi_{{\underline{\widetilde{\Delta}^{+}}}^{+}}^{a}\left(\mathscr{O}_{\widetilde{\mathscr{X}}_{\frac{o}{K}}}\right)\right) \rightarrow i_{*}\left(i^{*} G \otimes \Phi_{\underline{\widetilde{\Delta}}^{+}}^{a}\left(\mathscr{O}_{\mathscr{Y}_{\frac{o}{K}}}\right)\right)(-1)[-1] \stackrel{+1}{\rightarrow}$

in the almost derived category of sheaves in $\mathscr{X}_{\bar{K}}^{o \widetilde{\Delta}^{+}}$. 
Let $\mathscr{J} \widetilde{\underline{\Delta}}^{+}$denote the sheaf in $\mathscr{X}_{\bar{K}}^{o \widetilde{\Delta}^{+}}$whose -1 -component is $\mathscr{J}$ (the ideal defining the boundary) and whose components for $r \geq 0$ are zero, and similarly define $\mathscr{J}^{\widetilde{\underline{\Delta}}^{+}}$and $\mathscr{J}_{\bar{Y}}^{\widetilde{\widetilde{\Delta}}^{+}}$in $\widetilde{\mathscr{X}}_{\bar{K}}^{o \widetilde{\Delta}^{+}}$and $\mathscr{Y}_{\bar{K}}^{o \widetilde{\underline{\Delta}}^{+}}$respectively. We then have maps

$$
\mathscr{J}^{\widetilde{\underline{\Delta}}^{+}} \rightarrow \Phi_{\widetilde{\underline{\Delta}}^{+}}^{a}\left(\mathscr{O}_{\mathscr{X}_{\bar{K}}^{o}}\right), \quad \widetilde{\mathscr{J}}^{\widetilde{\Delta}^{+}} \rightarrow \Phi_{\underline{\widetilde{\underline{\Delta}}}^{+}}^{a}\left(\mathscr{O}_{\widetilde{\mathscr{X}}_{\bar{K}}}\right), \quad \mathscr{J}_{\widetilde{\widetilde{Y}}^{+}} \rightarrow \Phi_{\underline{\widetilde{\underline{\Delta}}}^{+}}^{a}\left(\mathscr{O}_{\mathscr{Y} \frac{o}{K}}\right)
$$

inducing the maps

$$
\mathscr{J} \rightarrow \Phi^{a}\left(\mathscr{O}_{\mathscr{X}_{\bar{K}}^{o}}\right), \quad \widetilde{\mathscr{J}} \rightarrow \Phi^{a}\left(\mathscr{O}_{\widetilde{\mathscr{X}}_{\bar{K}}^{o}}\right), \quad \mathscr{J}_{Y} \rightarrow \Phi^{a}\left(\mathscr{O}_{\mathscr{Y}_{\frac{o}{K}}}\right)
$$

defined in 8.15 .5 by normalization. It follows from the construction of the distinguished triangles 8.22 .1 and 8.34 .2 that the diagram

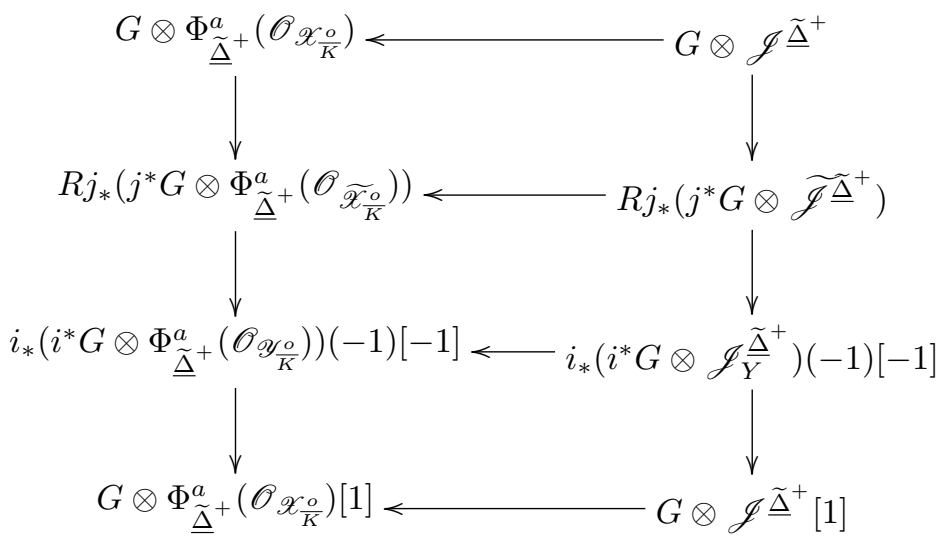

commutes and becomes an isomorphism between the two distinguished triangles, after applying $R \nu_{*}$.

Note also that if $\Lambda=\mathbb{Z} / p^{r} \rightarrow \Phi_{\widetilde{\Delta}^{+}}^{a}\left(\mathscr{O}_{\mathscr{X}_{K}^{o}}\right) / p^{r}$ is the unique morphism of rings in $\mathscr{X}_{\bar{K}}^{o \widetilde{\Delta}^{+}}$(where $\Lambda$ denotes the ring which in every degree is $\Lambda$ ), then there is a canonical map

$$
\Psi_{\underline{\underline{\underline{x}}}^{+}}^{a}(\Lambda) \rightarrow \mathbb{Z} / p^{r} \otimes \Phi_{\underline{\widetilde{\underline{O}}}^{+}}^{a}\left(\mathscr{O}_{\mathscr{X}_{\frac{\mathrm{K}}{o}}}\right) .
$$

This defines for every sheaf $G$ of $\Lambda$-modules a morphism

$$
\Psi^{a}(G) \rightarrow G \otimes \Psi_{\widetilde{\underline{\Delta}}^{+}}^{a}(\Lambda) \rightarrow G \otimes \Phi_{\underline{\underline{\unlhd}}^{+}}^{a}\left(\mathscr{O}_{\mathscr{X}_{\frac{\mathrm{K}}{K}}}\right) .
$$

It follows from the construction that this map induces 8.18 .3 by normalization.

8.35. Next we need the analogue of the sequence 8.31.1 for the sheaves $\Psi^{a}(F)$ in $\mathscr{X}_{K} \frac{}{K}$.

Let $U \rightarrow X$ be either an étale morphism such that the conditions of 7.1 hold (i.e. each of the $D_{A}^{o}$ as well as $\widetilde{U}_{\bar{K}}:=U \times_{X} \widetilde{X}_{\bar{K}}^{o}$ are $K(\pi, 1)$ 's) or the strict henselization of $X$ at a geometric point (in which case the conditions of 7.1 as well as condition (R) with respect to $D \cup Y$ hold). Let $Y_{U} \subset U$ be the inverse image of $Y$.

Let $\Delta$ denote the fundamental group of $U_{\bar{K}}^{o}$ (with respect to a geometric generic point), $\widetilde{\Delta}$ the fundamental group of $\widetilde{U}_{\bar{K}}$, let $\Delta_{Y_{U}}$ denote the fundamental group of $Y_{U, \bar{K}}^{o}$ and the choice of a specialization morphism defines a homomorphism $\Delta_{Y_{U}} \rightarrow$ $\Delta$ (well-defined up to conjugation). If condition (R) with respect to $D \cup Y$ holds then this map $\Delta_{Y_{U}} \rightarrow \Delta$ is an inclusion. 
Consider the diagram of topoi

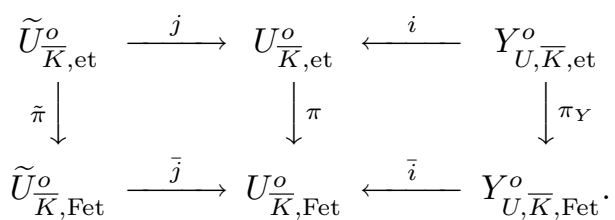

LEMmA 8.36. Let $F$ be a sheaf of $\Lambda$-modules in $U_{\bar{K}}^{o}$,Fet. Then there is a canonical isomorphism

$$
R^{1} \bar{j}_{*} \bar{j}^{*} F \simeq \bar{i}_{*} \bar{i}^{*} F(-1) .
$$

If condition (R) holds then $R^{s} \bar{j}_{*} \bar{j}^{*} F=0$ for all $s>1$.

Proof. Since $\widetilde{U}_{\bar{K}}^{o}$ is a $K(\pi, 1)$ we have

$$
R^{s} \bar{j}_{*} \bar{j}^{*} F \simeq R^{s}(\pi \circ j)_{*} j^{*} \pi^{*} F,
$$

and since $Y_{U, \bar{K}}^{o}$ is a $K(\pi, 1)$ we have

$$
R^{s} \bar{i}_{*} \bar{i}^{*} F \simeq R^{s} \pi_{*}\left(i_{*} i^{*} \pi^{*} F\right) .
$$

The purity triangle on $U_{\bar{K}, \text { et }}^{o}$

$$
\pi^{*} F \rightarrow R j_{*} j^{*} \pi^{*} F \rightarrow i_{*} i^{*} \pi^{*} F(-1)[-1]
$$

induces an exact sequence

$$
R^{1} \pi_{*} \pi^{*} F \rightarrow R^{1}(\pi \circ j)_{*} j^{*} \pi^{*} F \rightarrow \pi_{*} i_{*} i^{*} \pi^{*} F(-1) \rightarrow R^{2} \pi_{*} \pi^{*} F .
$$

Since $U_{\bar{K}}^{o}$ is a $K(\pi, 1)$ we have $R^{i} \pi_{*} \pi^{*} F=0$ for $i>0$ which implies the first part of the lemma, and in fact shows that for $s \geq 1$ we have

$$
R^{s} \bar{j}_{*} \bar{j}^{*} F \simeq R^{s-1} \bar{i}_{*} \bar{i}^{*} F(-1) .
$$

If condition (R) holds then $\bar{i}_{*}$ is given in terms of group cohomology by induction via the inclusion $\Delta_{Y_{U}} \hookrightarrow \Delta$ which is an exact functor. Hence the higher cohomology groups vanish in this case.

COROLlary 8.37. Let $\Sigma$ denote the kernel of the surjective homomorphism $\widetilde{\Delta} \rightarrow \Delta$. There is a canonical isomorphism of $\Delta$-representations

$$
H^{1}(\Sigma, \Lambda) \simeq \operatorname{Ind}_{\Delta_{Y_{U}}}^{\Delta}(\Lambda)(-1) .
$$

Proof. For a $\Delta$-representation $M$ let $M^{\sim}$ denote the corresponding sheaf on $U_{\mathrm{Fet}}^{o}$. We then have

$$
\begin{aligned}
H^{1}(\Sigma, \Lambda)^{\sim} & \simeq R^{1} \bar{j}_{*} \Lambda \\
& \simeq \bar{i}_{*} \Lambda(-1) \\
& \simeq \operatorname{Ind}_{\Delta_{Y_{U}}}^{\Delta}(\Lambda)^{\sim}(-1)
\end{aligned}
$$

8.38. Let $I_{A} \subset H_{A} \subset \Delta$ and $\widetilde{I}_{A} \subset \widetilde{H}_{A} \subset \widetilde{\Delta}$ be the decomposition and inertia groups of $D_{A} \subset U$ and $\widetilde{D}_{A}:=D_{A} \cap \widetilde{U} \subset \widetilde{U}$.

The structure of the inertia groups is very simple. Write $U_{\bar{K}}=\operatorname{Spec}(R)$, and let $\bar{R}$ be the integral closure of $R$ in the compositum of all finite extensions $L$ of $\operatorname{Frac}(R)$ (in the fixed geometric generic point) for which the normalization of $R$ in $L$ is étale over $U_{\bar{K}}^{o}$. The decomposition groups $H_{A}$ is defined by the choice of a 
geometric point $\bar{y} \rightarrow \operatorname{Spec}(\bar{R})$ mapping to the generic point of $D_{A}^{o}$. The inertia group $I_{A}$ is equal to the subgroup of $\Delta$ of elements which fix $\bar{y}$ and act trivially on the residue field of $\mathscr{O}_{\operatorname{Spec}(\bar{R}), \bar{y}}$. Let $\widehat{\mathscr{O}}_{R, \bar{y}}$ denote the completion of the strict henselization of $R$ at $\bar{y}$ and let $\widetilde{R}_{\bar{y}}$ denote the local ring of $\bar{y}$ in $\operatorname{Spec}\left(\bar{R} \otimes_{R} \widehat{\mathscr{O}}_{R, \bar{y}}\right)$. If $|A|=r$, then $\widehat{R}_{\bar{y}}$ is isomorphic to $S\left[\left[t_{1}, \ldots, t_{r}\right]\right]$ for some $\operatorname{ring} S$ with $D_{A}$ defined by $t_{1}=t_{2}=\cdots=t_{r}=0$. The extension $\widetilde{R}_{\bar{y}}$ is then by Abhyankar's lemma equal to the extensions obtained by taking roots of the variables $t_{i}$. This defines an isomorphism $I_{A} \simeq \widehat{\mathbb{Z}}(1)^{r}$. Applying the same analysis to $\widetilde{U}$ and $Y_{U}$ we obtain:

CoROLlary 8.39. The projection $\widetilde{I}_{A} \rightarrow I_{A}$ is an isomorphism. The projection $\Sigma \cap \widetilde{H}_{A} \rightarrow \widetilde{H}_{A} / \widetilde{I}_{A}$ is injective and identifies $\Sigma \cap \widetilde{H}_{A}$ with the kernel of the map $\widetilde{H}_{A} / \widetilde{I}_{A} \rightarrow H_{A} / I_{A}$. In particular, if $\Sigma_{A}$ denotes the kernel of the homomorphism $\widetilde{\Delta}_{A} \rightarrow \Delta_{A}$ (where $\Delta_{A}$ denotes the fundamental group of $D_{A, \bar{K}}^{o}$ etc.), then we have a commutative diagram

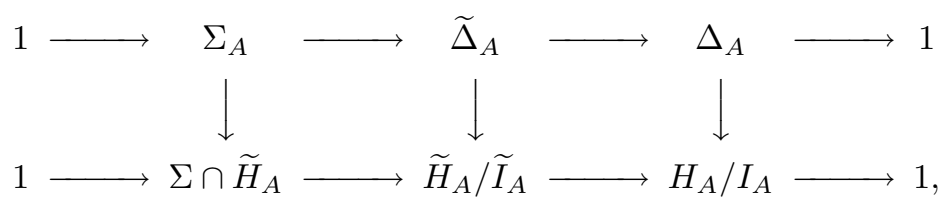

where the rows are exact and the vertical maps are surjective.

If condition $(R)$ holds, then the vertical maps in 8.39.1 are isomorphisms, and there is a commutative diagram with exact rows

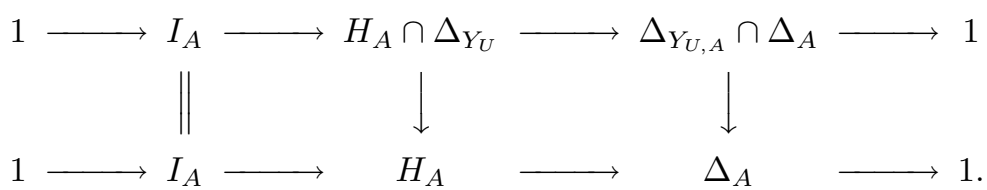

Corollary 8.40. Let $H_{A, Y}$ denote the decomposition group of $D_{A} \cap Y_{U} \subset Y_{U}$, and let $\Delta_{Y_{U}, A}$ denote the fundamental group of $D_{A}^{o} \cap Y_{U}^{o}$. The natural map of $\Delta_{A}$-representations

$$
\operatorname{Ind}_{H_{A, Y}}^{H_{A}}(\Lambda) \simeq \operatorname{Ind}_{H_{A, Y} / I_{A}}^{H_{A} / I_{A}}(\Lambda) \rightarrow \operatorname{Ind}_{\Delta_{Y_{U, A}}^{A}}^{\Delta_{A}}(\Lambda)
$$

is an isomorphism if condition $(R)$ holds.

COROLLARY 8.41. There is a canonical morphism

$$
H^{1}\left(\Sigma \cap \widetilde{H}_{A}, \Lambda\right) \rightarrow \operatorname{Ind}_{\Delta_{Y_{U}, A}}^{\Delta_{A}}(\Lambda)(-1)
$$

which is an isomorphism if condition $(R)$ holds. Furthermore, if $(R)$ holds then $H^{s}\left(\Sigma \cap \widetilde{H}_{A}, \Lambda\right)=0$ for all $s>1$.

Proof. Applying 8.37 to

$$
\Sigma_{A}:=\operatorname{Ker}\left(\widetilde{\Delta}_{A} \rightarrow \Delta_{A}\right)
$$

we obtain an isomorphism

$$
H^{1}\left(\Sigma_{A}, \Lambda\right) \simeq \operatorname{Ind}_{\Delta_{Y_{U}, A}}^{\Delta_{A}}(\Lambda)(-1) .
$$

From the map $\Sigma_{A} \rightarrow \Sigma \cap \widetilde{H}_{A}$ we then obtain a morphism

$$
H^{1}\left(\Sigma \cap \widetilde{H}_{A}, \Lambda\right) \rightarrow H^{1}\left(\Sigma_{A}, \Lambda\right) \simeq \operatorname{Ind}_{\Delta_{Y_{U}, A}}^{\Delta_{A}}(\Lambda)(-1) .
$$


That it is an isomorphism when $(\mathrm{R})$ holds follows from 8.40 and the fact that when (R) holds the map $\Sigma_{A} \rightarrow \Sigma \cap \widetilde{H}_{A}$ is an isomorphism. The last statement follows from 8.36 .

8.42. Now let $F$ be an injective sheaf in $U_{\bar{K}}^{o}$, Fet and consider the sheaf $\psi_{A}(F)$ on $U_{\bar{K}, \text { Fet }}^{o}$. Recall (see 7.11 that if $F$ corresponds to an injective $\Delta$-representation $M$ then $\psi_{A}(F)$ corresponds to the induced representation $\operatorname{Ind}_{H_{A}}^{\Delta}\left(\left.M\right|_{H_{A}}\right)$. Consider again the diagram

$$
\widetilde{U}_{\bar{K}, \text { Fet }}^{o} \stackrel{\bar{j}}{\longrightarrow} U_{\bar{K}, \text { et }}^{o} \stackrel{\bar{i}}{\longleftarrow} Y_{U, \bar{K}, \text { Fet }}^{o}
$$

The group $H^{s}\left(\widetilde{U}_{\bar{K}, \text { Fet }}, \psi_{A}\left(j^{*} F\right)\right)$ is equal to

$$
H^{s}\left(\widetilde{H}_{A}, M\right) \text {. }
$$

The Leray spectral sequence corresponding to $\widetilde{H}_{A} \rightarrow H_{A}$ gives a spectral sequence

$$
H^{p}\left(H_{A}, H^{q}\left(\Sigma \cap \widetilde{H}_{A}, M\right)\right) \Longrightarrow H^{p+q}\left(\widetilde{H}_{A}, M\right),
$$

which we can also write as

$$
H^{p}\left(H_{A}, M \otimes H^{q}\left(\Sigma \cap \widetilde{H}_{A}, \Lambda\right)\right) \Longrightarrow H^{p+q}\left(\widetilde{H}_{A}, M\right)
$$

since $M$ is a flat $\Lambda$-module by 7.15 .

In particular, for $s=1$ we have $H^{1}\left(H_{A}, M\right)=0$ since $M$ is injective so we get an isomorphism

$$
H^{1}\left(\widetilde{H}_{A}, M\right) \simeq H^{0}\left(H_{A}, M \otimes H^{1}\left(\Sigma \cap \widetilde{H}_{A}, \Lambda\right)\right) .
$$

We then obtain a diagram

$$
\begin{gathered}
H^{1}\left(\widetilde{H}_{A}, M\right) \longrightarrow H^{0}\left(\Delta_{A}, M^{I_{A}} \otimes \operatorname{Ind}_{\Delta_{Y_{U}, A}}^{\Delta_{A}}(\Lambda)(-1)\right) \\
H^{0}\left(H_{A}, M \otimes \operatorname{Ind}_{H_{A, Y}}^{H_{A}}(\Lambda)(-1)\right) \\
\simeq \uparrow \\
H^{0}\left(H_{A, Y}, M(-1)\right) .
\end{gathered}
$$

If condition $(\mathrm{R})$ holds then all these maps are isomorphisms.

8.43. Let $F$ be an injective sheaf in $\mathscr{X}_{\frac{O}{K}}^{o}$ and consider the diagram

$$
\widetilde{\mathscr{X}}_{\bar{K}}^{o} \underline{\widetilde{\Delta}}^{+} \stackrel{j}{\longrightarrow} \mathscr{X}_{\frac{o}{K}}^{o \widetilde{\Delta}^{+}} \stackrel{i}{i} \mathscr{Y}_{\bar{K}}^{o \widetilde{\Delta}^{+}} \text {. }
$$

Let

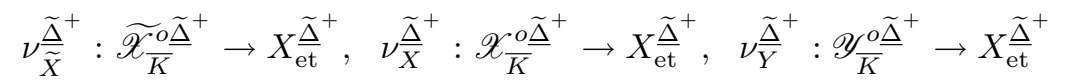

be the projections.

LEMMA 8.44. We have canonical isomorphisms

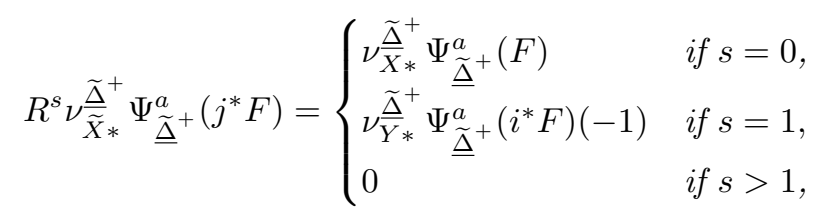


and

for $t>0$.

$$
R^{t} \nu_{\bar{Y}^{*}}^{\widetilde{\Delta}^{+}} \Psi_{\widetilde{\Delta}^{+}}^{a}\left(i^{*} F\right)=0
$$

Proof. Fix $[r] \in \underline{\widetilde{\Delta}}^{+}$. The restriction of $R^{s} \nu_{\widetilde{\widetilde{X}}^{+}}^{+} \Psi_{\widetilde{\underline{\Delta}}^{+}}^{a}\left(j^{*} F\right)$ to the $[r]$-component of $X_{\text {et }}^{\widetilde{\widetilde{\Delta}}^{+}}$is the sheaf $R^{s} \nu_{\widetilde{X} *} \Psi_{\widetilde{\Delta}^{+}}^{a}\left(j^{*} F\right)_{r}$ where $\Psi_{\widetilde{\underline{\Delta}}^{+}}^{a}\left(j^{*} F\right)_{r}$ is the sheaf on $\widetilde{\mathscr{X}_{\bar{K}}^{o}}$ associated to the presheaf whose restriction to $\widetilde{U}_{\bar{K}}^{o}$, Fet is equal to

$$
\oplus_{A \subset\{1, \ldots, m\},|A|=r} \psi_{A}\left(j^{*} F_{U}\right),
$$

and

$$
\nu_{\widetilde{X}}: \widetilde{\mathscr{X}_{\bar{K}}^{o}} \rightarrow X_{\text {et }}
$$

is the natural morphism of topoi. By 6.9 the sheaf $R^{s} \nu_{\widetilde{X} *} \Psi_{\widetilde{\Delta}^{+}}^{a}\left(j^{*} F\right)_{r}$ is equal to the sheaf associated to the presheaf which to any étale $U \rightarrow X$, satisfying the conditions in 7.1 assigns

$$
\oplus_{A \subset\{1, \ldots, m\},|A|=r} H^{s}\left(\widetilde{H}_{A}, M_{U}\right),
$$

where $\widetilde{H}_{A}$ is as in 8.38 and $M_{U}$ denotes the $\Delta$-representation corresponding to $F_{U}$. From this the case $s=0$ follows immediately, and the vanishing for $s>1$ follows from 8.41 by a standard limit argument.

Similarly the sheaf $R^{t} \nu_{\bar{\Upsilon}^{*}}^{\widetilde{\Delta}^{+}} \Psi_{\widetilde{\Delta}^{+}}^{a}\left(i^{*} F\right)_{r}$ is equal to the sheaf associated to the presheaf which to $U \rightarrow X$ as above associates

$$
\oplus_{A \subset\{1, \ldots, m\},|A|=r} H^{t}\left(H_{A, Y}, M_{U}\right) .
$$

From this description, a limit argument again, and 7.18 it follows that

for $t>0$.

$$
R^{t} \nu_{\overline{\underline{\Delta}}^{*}}^{\widetilde{\widetilde{\Delta}}^{+}} \Psi_{\widetilde{\underline{\Delta}}^{+}}^{a}\left(i^{*} F\right)_{r}=0
$$

Furthermore the maps in 8.42 .6 and a limit argument using the fact that for any geometric point $\bar{x} \rightarrow X$ condition (R) holds for $\operatorname{Spec}\left(\mathscr{O}_{X, \bar{x}} \otimes_{V} \bar{K}\right)$ yield the isomorphism

$$
R^{1} \nu_{\widetilde{\widetilde{X}}_{*}^{+}}^{\widetilde{\widetilde{\Delta}}^{+}} \Psi_{\widetilde{\underline{\Delta}}^{+}}^{a}\left(j^{*} F\right)_{r} \simeq \nu_{\overline{\mathrm{S}}^{*}}^{\widetilde{\widetilde{\Delta}}^{+}} \Psi_{\widetilde{\underline{\Delta}}^{+}}^{a}\left(i^{*} F\right)(-1)_{r} .
$$

We leave to the reader the verification that these isomorphisms are compatible with the simplicial structure, as $r$ varies.

Corollary 8.45. Let $F$ be a sheaf of $\Lambda$-modules in $\mathscr{X}_{\bar{K}}$. Then there is a canonical distinguished triangle in $X_{\text {et }}^{\widetilde{\widehat{\Delta}}^{+}}$

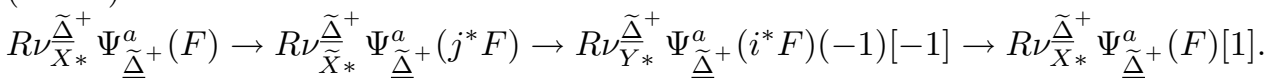

In particular, we obtain by normalization a distinguished triangle in $X_{\mathrm{et}}$

$$
R \nu_{X *} \Psi^{a}(F) \rightarrow R \nu_{\tilde{X}_{*}} \Psi^{a}\left(j^{*} F\right) \rightarrow R \nu_{Y *} \Psi^{a}\left(i^{*} F\right)(-1)[-1] \rightarrow R \nu_{X *} \Psi^{a}(F)[1],
$$

where

$$
\nu_{\widetilde{X}}: \widetilde{\mathscr{X}_{\bar{K}}^{o}} \rightarrow X_{\mathrm{et}}, \quad \nu_{X}: \mathscr{X}_{\bar{K}}^{o} \rightarrow X_{\mathrm{et}}, \quad \nu_{Y}: \mathscr{Y} \frac{o}{K} \rightarrow X_{\mathrm{et}}
$$

are the projections. 
Proof. In the case when $F$ is an injective sheaf of $\Lambda$-modules this follows from 8.44

To show the general case, it suffices to construct the triangle for a bounded below complex $F^{\bullet}$ of injective sheaves in $\mathscr{X}_{\bar{K}}^{o}$, instead of just a single injective sheaf. To handle this case, choose a bicomplex $J^{\bullet \bullet}$ of injectives in $\widetilde{\mathscr{X}_{K}^{o}}$ and a map of bicomplexes $j^{*} F^{\bullet} \rightarrow J^{\bullet \bullet}$ such that for every $j$ the map $j^{*} F^{j} \rightarrow J^{j \bullet}$ is a quasiisomorphism. Let $\tau_{\geq 1} \nu_{\tilde{X}_{*} *} \Psi_{\widetilde{\Delta}^{+}}\left(J^{\bullet \bullet}\right)$ be the truncation with respect to the second index. We then obtain from 8.44 a quasi-isomorphism

$$
\tau_{\geq 1} \nu_{\widetilde{X}_{*}} \Psi_{\widetilde{\underline{\Delta}}^{+}}^{a}\left(J^{\bullet \bullet}\right) \simeq \nu_{Y *} \Psi_{\underline{\widetilde{\underline{S}}}^{+}}^{a}\left(i^{*} F^{\bullet}\right)
$$

defining the desired triangle 8.45 .1 .

8.46. For any sheaf $F$ of $\Lambda$-modules in $\mathscr{X}_{\bar{K}}^{o}$ we obtain a diagram

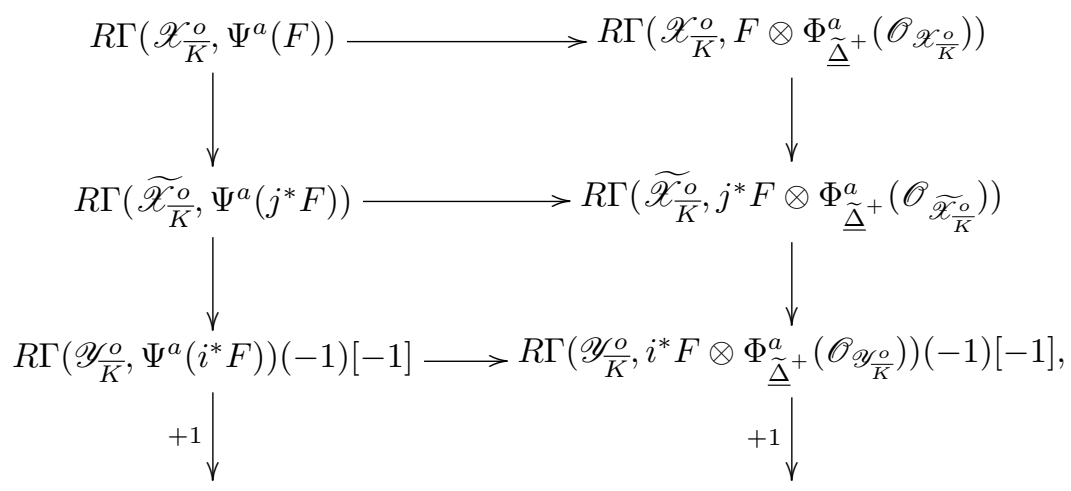

where the left triangle is obtained from 8.45.2, the right triangle is obtained from 8.34 .2 , and the horizontal arrows are the maps 8.34.7. It follows from the construction that this diagram commutes.

8.47. Let $L$ be a locally constant constructible sheaf on $X \bar{K}$, and let $\mathscr{L}:=u_{X *} L$ denote the induced sheaf on $\mathscr{X}_{K}^{o}$. Choose an injective resolution $\mathscr{L} \rightarrow I^{\bullet}$. For a hypercover $U . \in H R(X)^{\prime}$ such that each $U_{n, \bar{K}}$ also satisfies the conditions of 7.1 with respect to the divisor $D \cup Y$, we then have by 8.31.1 a distinguished triangle

$$
\bar{\Psi}\left(\left.I^{\bullet}\right|_{U^{\circ}, \bar{K}}\right) \rightarrow R j_{*} \bar{\Psi}\left(\left.I^{\bullet}\right|_{\widetilde{U}_{\cdot, \bar{K}}^{o}}\right) \rightarrow i_{*} \bar{\Psi}\left(\left.I^{\bullet}\right|_{U_{,, Y, \bar{K}}^{o}}\right)(-1)[-1] \rightarrow \bar{\Psi}\left(\left.I^{\bullet}\right|_{U^{\circ, \bar{K}}} ^{o}\right)[1]
$$

on $U_{\cdot, \bar{K}, \text { et }}$, where $U_{\cdot, Y}$ denotes the base change $U . \times_{X} Y$ and $\left.I^{\bullet}\right|_{U^{o}, \bar{K}}$ denotes the pushforward of $I^{\bullet}$ to $U_{\cdot, \bar{K}, \text { Fet }}^{o}$. 
We obtain a diagram

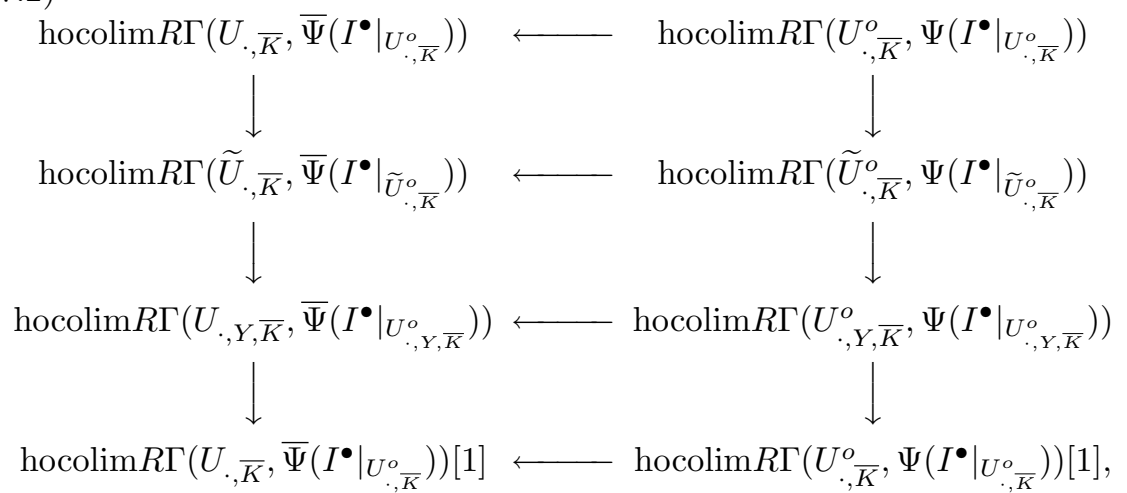

where the columns are the distinguished triangles obtained from 8.47.1 and 8.45.2, and the horizontal arrows are the morphisms obtained from 8.8.1, and the homotopy colimits are taken over $U . \in H R(X)^{\prime}$.

Proposition 8.48. The diagram 8.47.2 commutes.

Proof. Let pt denote the punctual topos, and let $T$ denote the topos $(\mathrm{pt} \underline{\underline{\Delta}}) \underline{\widetilde{\Delta}}^{+}$. So a $\Lambda$-module in $T$ consists of a $\Lambda$-module $F_{n, m}$ for every pair of objects $[n] \in \Delta$ and $[m] \in \widetilde{\Delta}^{+}$together with transition morphisms. We refer to $F_{n, m}$ as the $(n, m)$ component of $F$.

For a simplicial scheme $Z$. there is a canonical morphism of topoi

$$
q: Z{\stackrel{\tilde{\Delta}^{+}}{\rightarrow}}^{+} T
$$

For a sheaf $F \in Z^{\widetilde{\Delta}^{+}}$we write $\widetilde{\Gamma}(F)$ for $q_{*} F$ and $R \widetilde{\Gamma}(F)$ for $R q_{*} F$. Taking the total complex (in both the $\Delta$-direction and $\widetilde{\Delta}^{+}$-direction) defines a triangulated functor

$$
\text { Tot }: D(T, \Lambda) \rightarrow D(\Lambda) \text {. }
$$

The diagram 8.47 .2 is obtained by applying Tot to a diagram in $D(T, \Lambda)$. Namely, we have complexes of sheaves $\Psi_{\widetilde{\underline{\Delta}}^{+}}\left(I^{\bullet}\right)$ and $\bar{\Psi}_{\widetilde{\underline{\Delta}}^{+}}\left(I^{\bullet}\right)$ in $U_{\cdot, \overline{\bar{K}}}^{o \widetilde{\widetilde{\Delta}}^{+} \text {Fet }}$ and $U_{\cdot, \bar{K} \text {, et }}^{\widetilde{\widetilde{\Delta}}^{+}}$ respectively. The diagram 8.47 .2 is then obtained by applying Tot to the diagram (8.48.3)

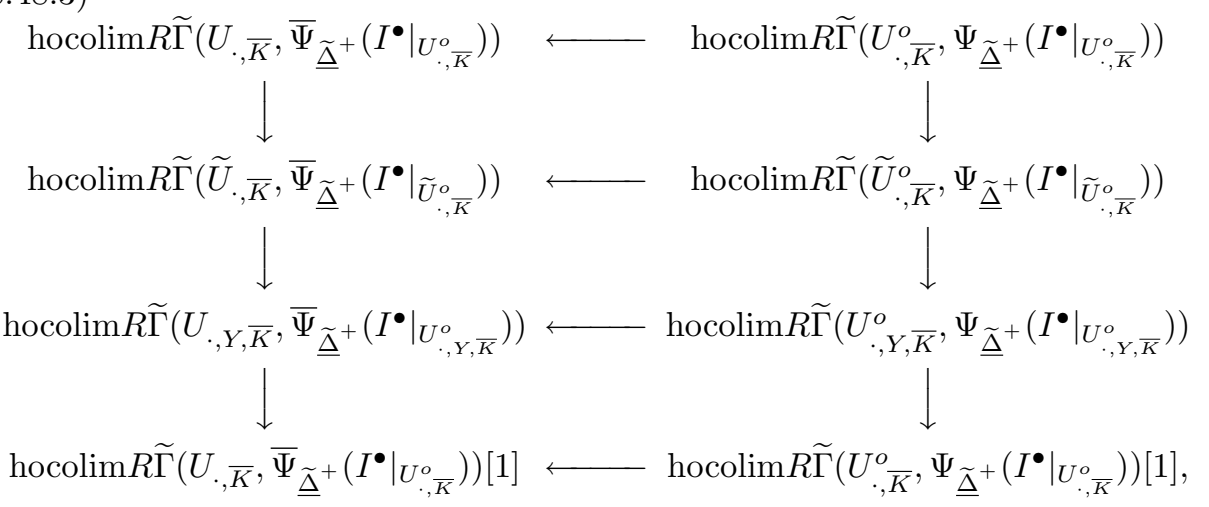

where the homotopy colimits are taken over $U . \in H R(X)^{\prime}$. 
Choose a bicomplex $J^{\bullet \bullet}$ of injective objects in $\widetilde{\mathscr{X}_{\bar{K}}^{o}}$ and a morphism $\left.I^{\bullet}\right|_{\widetilde{\mathscr{X}_{K}} \frac{o}{K}} \rightarrow$ $J^{\bullet \bullet}$ such that for every $j$ the map

$$
I^{j} \rightarrow J^{j \bullet}
$$

is a quasi-isomorphism. For a hypercover $U$. $\in H R(X)^{\prime}$ and for any $j \geq 0$, the complex in $T$

$$
\widetilde{\Gamma}\left(\widetilde{U}_{\cdot, \bar{K}, \mathrm{Fet}}^{o}, \Psi_{\widetilde{\underline{\Delta}}^{+}}\left(\left.J^{j \bullet}\right|_{U .}\right)\right)
$$

has $(n, m)$-component a complex computing the cohomology $R \Gamma\left(\widetilde{U}_{n, \bar{K}}^{o}, \mathrm{Fet}, \Psi\left(I^{j}\right)^{m}\right)$.

Also choose a cofinal projective system of étale hypercovers $\left\{U^{\alpha}\right\}$ of $X$.

From 8.36 we have:

LEMma 8.49. There are canonical isomorphisms

(i) $\lim _{\longrightarrow} R^{0} \Gamma\left(\widetilde{U}_{n, \bar{K}, \mathrm{Fet}}^{\alpha o}, \Psi\left(\left.I^{j}\right|_{\widetilde{U}_{\cdot \bar{K}}^{o}}\right)^{m}\right) \simeq \lim _{\longrightarrow} \Gamma\left(U_{n, \bar{K}, \mathrm{Fet}}^{\alpha o}, \Psi\left(I^{j}\right)^{m}\right)$,

(ii) $\lim _{\longrightarrow} R^{1} \Gamma\left(\widetilde{U}_{n, \bar{K}, \mathrm{Fet}}^{\alpha o}, \Psi\left(\left.I^{j}\right|_{\widetilde{U}_{\cdot, \bar{K}}^{o}}\right)^{m}\right) \simeq \lim _{\longrightarrow} \Gamma\left(Y_{n, \bar{K}, \mathrm{Fet}}^{\alpha o}, \Psi\left(\left.I^{j}\right|_{U_{,, Y, \bar{K}}^{o}}\right)^{m}\right)(-1)$,

and

(iii) for any $i>1$ we have

$$
\underset{\alpha}{\lim _{\alpha}} R^{i} \Gamma\left(\widetilde{U}_{n, \bar{K}, \text { Fet }}^{\alpha o}, \Psi\left(\left.I^{j}\right|_{\widetilde{U}_{\cdot, \bar{K}}^{o}}\right)^{m}\right)=0 .
$$

Let $\Theta\left(J^{j \bullet}\right)^{m}$ denote the kernel of the map

$$
\Psi\left(J^{j 1}\right)^{m} \rightarrow \Psi\left(J^{j 2}\right)^{m}
$$

induced by the morphism $J^{j 1} \rightarrow J^{j 2}$. By the above we then have an exact sequence (in the vertical direction) of complexes in $T$

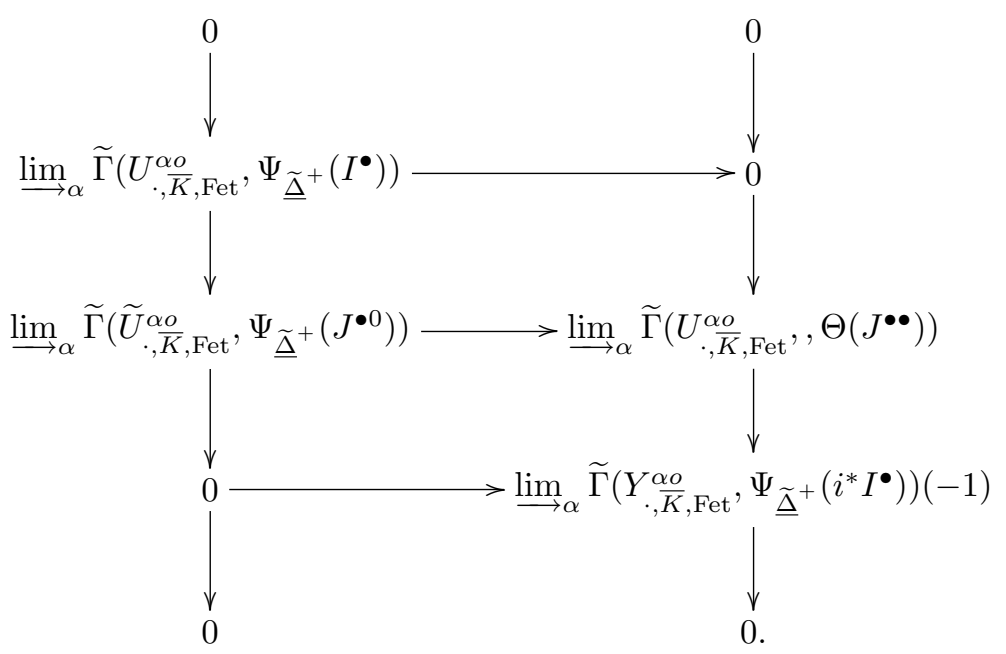

A very similar argument can be carried out with the complexes $\bar{\Psi}\left(I^{\bullet}\right)$ and $\bar{\Psi}\left(J^{\bullet \bullet}\right)$ (we leave the details to the reader). Let

$$
\bar{\Theta}\left(J^{j \bullet}\right)^{m}:=\operatorname{Ker}\left(\bar{\Psi}\left(J^{j 1}\right)^{m} \rightarrow \bar{\Psi}\left(J^{j 2}\right)^{m}\right) .
$$


We then also obtain an exact sequence of complexes in $T$

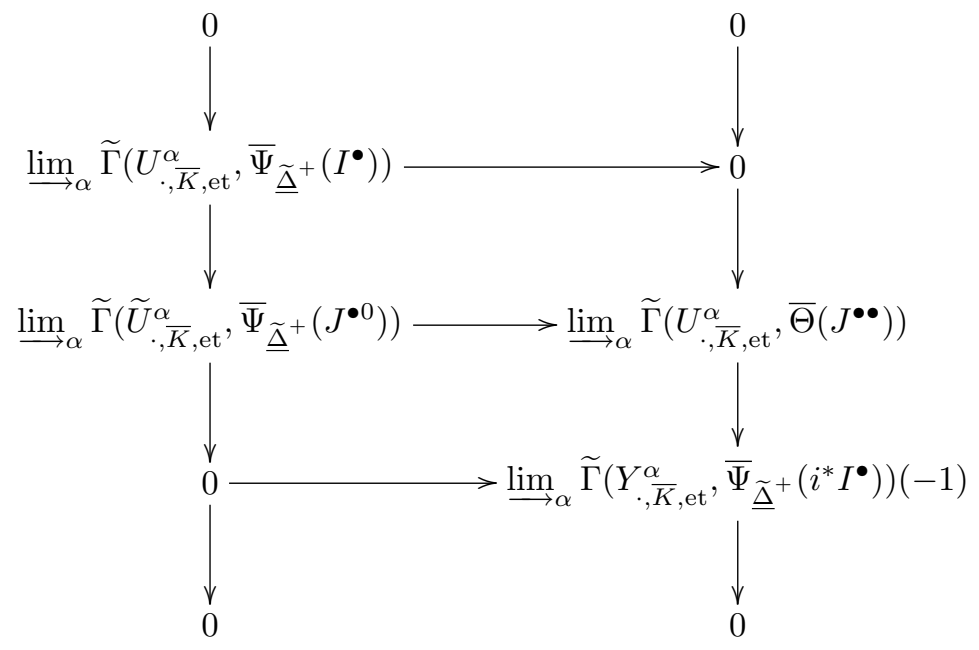

The construction of 8.8 .1 gives isomorphisms between the individual terms of 8.49 .1 and 8.49.4, and to prove 8.48 we have to check that in fact these maps give a morphism of diagrams (for then the diagram 8.47 .2 is obtained by taking total complexes).

To prove that we in fact get a morphism of diagrams from 8.49 .4 to 8.49 .1 , it suffices to show that for every $j \geq 0, m \geq-1$, and $n \geq 0$ the diagram

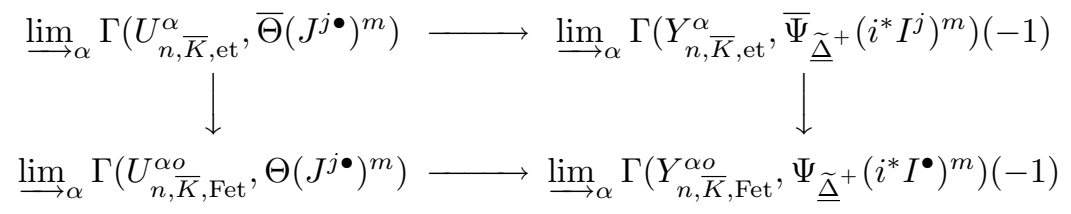

commutes (the other parts of the verification that we get a map of diagrams from 8.49 .4 to 8.49 .1 are immediate). Equivalently we need to show that the diagram

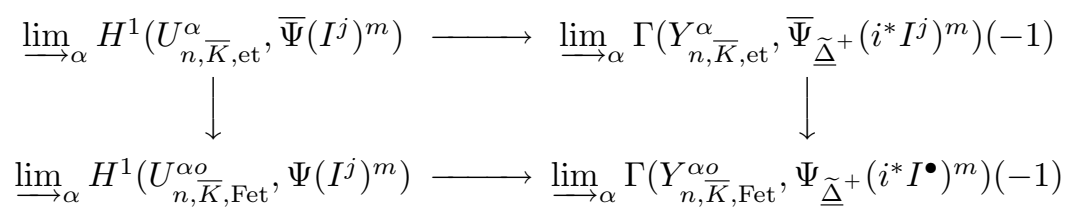

commutes, where the horizontal arrows are obtained from 8.49 and the vertical arrows are given by 8.8.1. The sheaves $\bar{\Psi}\left(I^{j}\right)^{m}$ and $\Psi\left(I^{j}\right)^{m}$ are by construction direct sums of sheaves

$$
\Psi\left(I^{j}\right)^{m}=\oplus \Psi_{A}\left(I^{j}\right)^{m}, \quad \bar{\Psi}\left(I^{j}\right)^{m}=\oplus \bar{\Psi}_{A}\left(I^{j}\right)^{m},
$$

and 8.49 .6 is obtained by taking the direct sum of diagrams

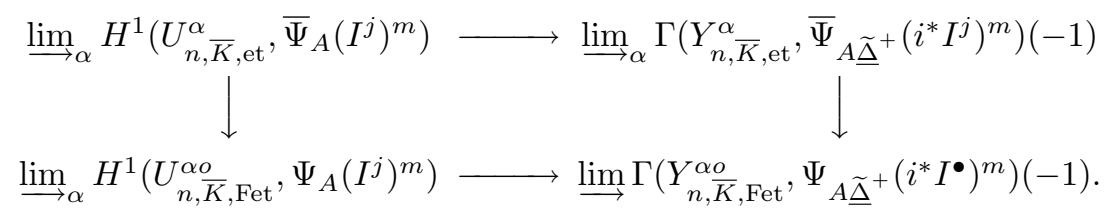


The proof of 8.48 is then completed by showing that 8.49 .8 commutes, which is a straightforward verification (which we leave to the reader). This completes the proof of 8.48 , and hence also of 8.21 in the case of a divisor.

8.50. To treat the case of a higher codimension inclusion $i: Y \hookrightarrow X$ we first need some computations.

If $\mathscr{L}$ is a line bundle on $X$ we define its first Chern class $c_{1}(\mathscr{L}) \in H^{2}\left(\mathscr{X}_{\bar{K}}^{o}, \mu_{p^{s}}\right)$ as follows. Raising to the $p^{s}$-th power induces an exact sequence

$$
0 \rightarrow \mu_{p^{s}} \rightarrow \mathscr{O}_{\mathscr{X}_{\bar{K}}^{\circ}}^{*} \stackrel{p^{s}}{\rightarrow} \mathscr{O}_{\mathscr{T}_{K}^{o}}^{*} \rightarrow 0,
$$

and we define $c_{1}(\mathscr{L})$ to be the image of the class of $\mathscr{L}$ under the induced map

$$
H^{1}\left(X, \mathscr{O}_{X}^{*}\right) \rightarrow H^{1}\left(\mathscr{X}_{\bar{K}}^{o}, \mathscr{O}_{\mathscr{X}_{K}^{\circ}}^{*}\right) \rightarrow H^{2}\left(\mathscr{X}_{\bar{K}}^{o}, \mu_{p^{s}}\right) .
$$

By 6.5 there is a canonical isomorphism $H^{2}\left(\mathscr{X}_{\bar{K}}^{o}, \mu_{p^{s}}\right) \simeq H^{2}\left(X_{\bar{K}}^{o},{ }_{\text {et }}, \mu_{p^{s}}\right)$ and it follows from the definition that under this isomorphism $c_{1}(\mathscr{L})$ is equal to the class obtained from the Kummer sequence $0 \rightarrow \mu_{p^{s}} \rightarrow \mathbb{G}_{m} \rightarrow \mathbb{G}_{m} \rightarrow 0$.

Computation 8.51. Let $X=\mathbb{P}_{V}^{n}$, and let $D$ be the union of the standard hyperplanes $\left\{x_{i}=0\right\}$. Let $\mathscr{C}$. be the Cech complex computing the coherent cohomology of $\mathscr{O}_{X}$ with respect to the standard covering. For any integer $n$, raising the coordinates to the $p^{n}$-th power defines a morphism

$$
\sigma_{n}: \mathbb{P}_{V}^{n} \rightarrow \mathbb{P}_{V}^{n},
$$

which induces a morphism

$$
\sigma_{n}^{*}: \mathscr{C}^{\cdot} \rightarrow \mathscr{C}
$$

The natural action of $\mu_{p^{n}}^{d}$ on $\mathbb{P}_{V}^{n}$ induces an action of $\Delta_{\infty}:=\mathbb{Z}_{p}(1)^{d}$ on $\lim _{n} \mathscr{C}$, where the limit is taken with respect to the maps $\sigma_{n}^{*}$. It follows from 3.10 , that we then have an almost isomorphism

$$
R \Gamma\left(\mathscr{X}_{\bar{K}}^{o}, \overline{\mathscr{O}} \mathscr{X}_{\bar{K}}^{o} / p^{s}\right) \simeq R \Gamma_{\Delta_{\infty}}\left(\underline{\lim }_{\longrightarrow} \mathscr{C} \cdot \otimes_{V} \bar{V} / p^{s}\right) .
$$

Since the natural map

$$
\bar{V} / p^{s} \rightarrow \lim _{\longrightarrow} \mathscr{C} \cdot \otimes_{V} \bar{V} / p^{s}
$$

is a quasi-isomorphism, we conclude from $3.5(\mathrm{i}))$ that there is an almost isomorphism

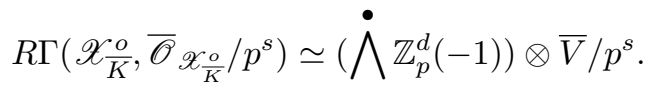

In the case when $n=1$, let $j: \mathbb{G}_{m} \hookrightarrow \mathbb{A}^{1}$ be the natural inclusion, and let $i: \operatorname{Spec}(V) \cup \operatorname{Spec}(V) \hookrightarrow \mathbb{P}^{1}$ be the inclusions of 0 and $\infty$. By 8.22 there is then a distinguished triangle in $\widetilde{D}\left(\mathscr{X}_{\bar{K}}\right)$

$$
\mathscr{O}_{\mathscr{X}_{\bar{K}}} \rightarrow R j_{*} \mathscr{O}_{\mathscr{X}_{\bar{K}}^{\circ}} \rightarrow i_{*} \mathscr{O}_{\mathscr{D}_{\bar{K}}^{\circ}}(-1)[-1] \rightarrow \mathscr{O}_{\mathscr{X}_{\bar{K}}}[1]
$$

where $D=D_{0} \amalg D_{\infty} \subset X$ denotes the inclusion $\operatorname{Spec}(V) \amalg \operatorname{Spec}(V) \hookrightarrow \mathbb{P}^{1}$ of 0 and $\infty$. By the above computation we know that $H^{*}\left(\mathscr{X}_{\bar{K}}, R j_{*} \mathscr{O}_{\mathscr{X}_{\bar{K}}}\right) \simeq \Lambda \bullet \bar{V} / p^{s}(-1)$ (almost isomorphism). Looking at the long exact sequence corresponding to 8.51.1 one sees that $H^{0}\left(\mathscr{X}_{\bar{K}}, \mathscr{O}_{\mathscr{X}_{\bar{K}}^{\circ}} / p^{s}\right) \simeq \bar{V} / p^{s}, H^{1}\left(\mathscr{X}_{\bar{K}}, \mathscr{O}_{\mathscr{X}_{\bar{K}}} / p^{s}\right)=0$, and

$$
H^{2}\left(\mathscr{X}_{\bar{K}}, \mathscr{O}_{\mathscr{X}_{\bar{K}}} / p^{s}\right) \simeq \bar{V} / p^{s}(-1)
$$

with generator the image under the boundary map of a generator of $H^{0}\left(\mathscr{D}_{0, \bar{K}}, \mathscr{O}_{\mathscr{D}_{K}}\right)$. A straightforward verification shows that this generator is equal to $-c_{1}\left(\mathscr{O}_{\mathbb{P}^{1}}(1)\right)$. 
Computation 8.52. Let $d \geq 1$ be an integer, and let $X=\mathbb{P}^{d}$. Denote by $H_{0}, \ldots, H_{d}$ the standard hyperplanes (so $H_{i}$ is the locus of points where the $i$-th coordinate is zero). For $0 \leq i \leq d$ let $\mathscr{X}_{\bar{K}, i}^{o}$ denote the topos obtained by taking $D=H_{0} \cup \cdots \cup H_{i}$. We claim that then $H^{*}\left(\mathscr{X}_{\bar{K}, i}^{o}, \overline{\mathscr{O}}_{X} / p^{s}\right)$ is isomorphic to the exterior algebra $\Lambda^{\bullet}\left(\bar{V} / p^{s}\right)^{i}(-1)$. This we prove by descending induction on $i$.

The case $i=d$ was done in the preceding computation.

For the general case note that the distinguished triangle 8.22 induces a long exact sequence

$$
\rightarrow H^{j}\left(\mathscr{X}_{\bar{K}, i}^{o}, \mathscr{O}_{\mathscr{X}_{\frac{o}{K}}}\right) \rightarrow H^{j}\left(\mathscr{X}_{\bar{K}, i+1}^{o}, \mathscr{O}_{\mathscr{X}_{\frac{o}{K}}}\right) \rightarrow H^{j-1}\left(\mathbb{P}^{d-1}-\{i \text { hyperplanes }\}\right)(-1) \rightarrow
$$

Using this and also induction on $d$ one obtains the general case.

Note in particular that for $D=H_{0}$ we find that $H^{i}\left(\mathscr{X}_{\bar{K}}^{o}, \overline{\mathscr{O}}_{X} / p^{s}\right)=0$ for $i>0$ and for $i=0$ we get $\bar{V} / p^{s}$. Using this we can also compute the cohomology of projective space $\mathbb{P}^{d}$ with $D=\emptyset$. We claim that in this case

$$
H^{*}\left(\mathscr{X}_{\bar{K}}, \mathscr{O}_{\mathscr{X}_{\bar{K}}} / p^{s}\right) \simeq \oplus_{i=0}^{d} \bar{V} / p^{s}(-i) \cdot \xi^{i}
$$

where $\xi \in H^{2}\left(\mathscr{X}_{\bar{K}}, \mathscr{O}_{\mathscr{X}_{\bar{K}}} / p^{s}\right)(1)$ denotes the Chern class of $\mathscr{O}_{X}(1)$. This is proven by induction on $d$, the case $d=0$ being trivial. For general $d$, let $X^{o} \operatorname{denote} X-H$ where $H$ is a hyperplane so that by 8.22 we have a long exact sequence

$$
\cdots \rightarrow H^{i}\left(\mathscr{X}_{\bar{K}}, \mathscr{O}_{\mathscr{X}_{\bar{K}}} / p^{s}\right) \rightarrow H^{i}\left(\mathscr{X}_{\bar{K}}^{o}, \mathscr{O}_{\mathscr{X}_{\bar{K}}^{o}} / p^{s}\right) \rightarrow H^{i-1}\left(\mathscr{H}_{\bar{K}}, \mathscr{O}_{\mathscr{H}_{\bar{K}}} / p^{s}\right)(-1) \rightarrow \cdots
$$

By the above we have $H^{i}\left(\mathscr{X}_{\frac{o}{K}}^{o} \mathscr{O}_{\mathscr{X}_{\frac{o}{K}}} / p^{s}\right)=0$ for $i>0$, and by the above the boundary map

$$
H^{i-1}\left(\mathscr{H}, \mathscr{O}_{\mathscr{H}_{\bar{K}}} / p^{s}\right)(-1) \rightarrow H^{i+1}\left(\mathscr{X}_{\bar{K}}, \mathscr{O}_{\mathscr{X}_{\bar{K}}} / p^{s}\right)
$$

is equal to $i_{*}$. Now we have $i_{*}\left(\xi^{j}\right)=\xi^{j+1}$. Indeed we have already shown that $i_{*}$ agrees with the étale pushforward $i_{*}^{\text {et }}$ for the inclusion of smooth divisors, so it suffices to verify this formula in the étale theory and by the projection formula it in turn suffices to show that $i_{*}(1)=\xi$ which is true for example by [9. Cycle, 2.9].

Proposition 8.53. Let $\mathscr{E}$ be a vector bundle of rank $r+1$ on $X$ and let $\pi$ : $Y=\mathbb{P}(\mathscr{E}) \rightarrow X$ be the corresponding projective bundle. Let $\widetilde{D} \subset Y$ be the inverse image of $D$, and for a decomposition $D=E \cup F$ let $\widetilde{D}=\widetilde{E} \cup \widetilde{F}$ be the decomposition obtained by taking inverse images of $E$ and $F$. Let $\mathscr{O}(1)$ denote the tautological line bundle on $Y$, and let $\xi \in H^{2}\left(\mathscr{Y}^{0}, \mu_{p^{s}}\right)$ denote the Chern class $c_{1}(\mathscr{O}(1))$. Then for any integer $s$ and sheaf $L$ the cup product map

$$
\oplus_{i=0}^{r} \cup \xi^{i}: \bigoplus_{i=0}^{r} H^{s-2 i}\left(\mathscr{X}_{\frac{o}{K}}^{o}, L \otimes \mathscr{J}_{E}\right)(-i) \rightarrow H^{s}\left(\mathscr{Y}_{\bar{K}}^{o}, \pi^{*} L \otimes \mathscr{J}_{\widetilde{E}}\right)
$$

is an almost isomorphism.

PROOF. It suffices to prove the proposition after making an étale localization on $X$. We may therefore consider the analogous statement for $X$ the spectrum of a strictly henselian local ring. In this case $Y$ is isomorphic to $X \times \mathbb{P}^{r}$. In this case the result follows from the computation 8.52 and the observation that if $P$ denotes $\mathbb{P}^{d}$ then $H^{*}\left(\mathscr{Y}^{o}, \mathscr{J}_{\widetilde{E}}\right) \simeq J_{E} \otimes_{V} H^{*}\left(\mathscr{P}_{\bar{K}}, \mathscr{O}_{\mathscr{P}_{\bar{K}}}\right)$, which follows from a similar argument to the one used in 8.52 using 3.16 
Corollary 8.54. With notation as in 8.53, the image of $\xi^{r}$ under the map

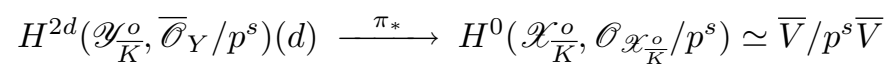

is equal to 1 .

Proof. Everything we have done is functorial with respect to morphisms $f$ : $\left(X^{\prime}, D^{\prime}\right) \rightarrow(X, D)$ such that $f^{-1}(D) \subset D^{\prime}$. After making a finite extension of $V$, we may assume that there exists a section $\operatorname{Spec}(V) \rightarrow X^{o}$. Base changing to $\operatorname{Spec}(V)$ it therefore suffices to consider the case when $X=\operatorname{Spec}(V)$ and $D$ is trivial so $Y=\mathbb{P}^{d}$.

We can now finally prove 8.21 in general. Let $i: Y \hookrightarrow X$ be as in the theorem and let $\widetilde{X}$ denote the blow-up of $X$ along $Y$. Let $\widetilde{D} \subset \widetilde{X}$ be the inverse image of $D$, and let $E \subset \widetilde{X}$ be the inverse image of $Y$ so we have a cartesian square

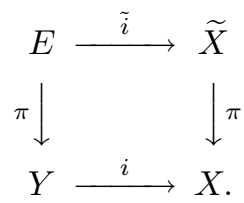

Let $r+1$ denote the codimension of $Y$ in $X$ so that $E$ is isomorphic to the projective bundle associated to the rank $r+1$-normal bundle $\mathscr{N}_{Y / X}$ on $Y$. Let $\xi \in H^{2 r}\left(E_{\bar{K}, \text { et }}^{o}, \mathbb{Z} / p^{s}(r)\right)$ denote the $r$-th power of the first Chern class of the tautological line bundle on $E=\mathbb{P}_{Y}\left(\mathscr{N}_{Y / X}\right)$. Since $\pi_{*} \xi=1$ (this follows for example from [24, VII.2.2.6]), the composite morphism

$$
\pi_{*}\left(\pi^{*}(-) \cup \xi\right): R \Gamma_{E_{Y}, F_{Y}}\left(Y_{\bar{K}, \mathrm{et}}^{0}, f^{*} u_{X}^{*} L\right) \rightarrow R \Gamma_{E_{Y}, F_{Y}}\left(Y_{\bar{K}, \mathrm{et}}^{0}, f^{*} u_{X}^{*} L\right)
$$

is the identity. Similarly, the morphism

$$
\pi_{*}\left(\pi^{*}(-) \cup \xi\right): R \Gamma\left(\mathscr{Y}_{K}^{o}, f^{*} L \otimes \mathscr{J}_{E_{Y}}\right) \rightarrow R \Gamma\left(\mathscr{Y}_{\bar{K}}^{o}, f^{*} L \otimes \mathscr{J}_{E_{Y}}\right)
$$

is the identity.

By [24, VII.8.4.3], the two maps $\pi^{*} i_{*}(-), \tilde{i}_{*}\left(\pi^{*}(-) \cup \xi\right)$

$$
R \Gamma_{E_{Y}, F_{Y}}\left(Y_{\bar{K}, \mathrm{et}}^{o}, f^{*} u_{X}^{*} L\right) \rightarrow R \Gamma_{E_{\widetilde{X}}, F_{\widetilde{X}}}\left(\widetilde{X}_{\bar{K}, \mathrm{et}}^{o}, \pi^{*} u_{X}^{*} L\right)(r+1)[2(r+1)]
$$

are equal. Since the transformations $\sigma_{Y}$ and $\sigma_{X}$ commute with $\tilde{i}_{*}$ by the case of a smooth divisor already treated we get that $\pi^{*} \sigma_{X}\left(i_{*}-\right)=\tilde{i}_{*}\left(\pi^{*}\left(\sigma_{Y}(-)\right) \cup \xi\right)$. Since $\pi_{*} \pi^{*}=$ id on $R \Gamma\left(\mathscr{X}_{K}^{o}, L \otimes \mathscr{J}_{E}\right)$ we can apply $\pi_{*}$ to obtain

$$
\sigma_{X}\left(i_{*}-\right)=\pi_{*} \tilde{i}_{*}\left(\pi^{*}\left(\sigma_{Y}(-)\right) \cup \xi\right)
$$

We then find that

$$
\begin{array}{rlc}
\sigma_{X}\left(i_{*}-\right) & =\pi_{*} \tilde{i}_{*}\left(\pi^{*}\left(\sigma_{Y}(-)\right) \cup \xi\right) \\
& = & i_{*} \pi_{*}\left(\pi^{*}\left(\sigma_{Y}(-)\right) \cup \xi\right) \\
& = & i_{*} \sigma_{Y}(-) .
\end{array}
$$

This completes the proof of 8.21

8.55. The next step in the proof of 6.16 is to prove some facts about the behavior of cohomology under blowups of the boundary. So let $D_{1}, D_{2} \subset D$ be two smooth divisors meeting tranversally, and let $\pi: \widetilde{X} \rightarrow X$ be the blow-up of their intersection. Let $\widetilde{D} \subset \widetilde{X}$ be the inverse image of $D$. The subscheme $\widetilde{D} \subset \widetilde{X}$ is also 
a divisor with simple normal crossings. In fact, $\widetilde{D}$ is equal to the union of the strict transforms of the components of $D$ together with the exceptional divisor $N \subset \widetilde{X}$.

Now let $D=E \cup F$ be a decomposition of the divisor $D$ such that $D_{1} \in E$ and $D_{2} \in F$, and let $\widetilde{D}=E_{1} \cup F_{1}$ be the decomposition obtained by taking $E_{1}$ equal to the union of the strict transforms of the divisors in $E$ together with $N$, and let $\widetilde{D}=E_{2} \cup F_{2}$ be the decomposition obtained by taking $E_{2}$ to be the union of the strict transforms of the components in $E$ (so $N \subset F_{2}$ ). In $\mathscr{X}_{\bar{K}}^{o}$ we then have morphisms

$$
\pi^{*} \mathscr{J}_{E} \rightarrow \mathscr{J}_{E_{2}} \leftarrow \mathscr{J}_{E_{1}} .
$$

Proposition 8.56. Let $L$ be a sheaf of flat $\Lambda$-modules in $\mathscr{X}_{\bar{K}}^{o}$. If $\pi: \widetilde{\mathscr{X}_{\bar{K}}^{o}} \rightarrow \mathscr{X}_{\bar{K}}^{o}$ is the induced morphism of topoi and $\nu_{X}: \mathscr{X}_{\bar{K}}^{o} \rightarrow X_{\text {et }}$ the projection, then the maps

$$
R \nu_{X *} L \otimes \mathscr{J}_{E} \rightarrow R \nu_{X *} R \pi_{*} \pi^{*} L \otimes \mathscr{J}_{E_{2}} \leftarrow R \nu_{X *} R \pi_{*} \pi^{*} L \otimes \mathscr{J}_{E_{1}} .
$$

are all almost isomorphisms. In particular the natural maps

$$
H^{*}\left(\mathscr{X}_{\bar{K}}^{o}, L \otimes \mathscr{J}_{E}\right) \rightarrow H^{*}\left(\widetilde{\mathscr{X}_{\bar{K}}^{o}}, \pi^{*} L \otimes \mathscr{J}_{E_{2}}\right) \leftarrow H^{*}\left(\widetilde{\mathscr{X}_{K}^{o}}, \pi^{*} L \otimes \mathscr{J}_{E_{1}}\right)
$$

are all almost isomorphisms.

Proof. We may work étale locally on $X$, and hence can assume that $X=$ $\operatorname{Spec}\left(V\left[X_{1}, \ldots, X_{r}, X, Y\right]\right)$ where $D$ is defined by $\left(X_{1} \cdots X_{r} X Y\right)$ and $\widetilde{X}$ is the blowup of the ideal $(X, Y)$ with $D_{1}=(X)$ and $D_{2}=(Y)$. We can further assume that $E=D_{1}$ and by the projection formula that $L$ is the trivial sheaf.

Write $R=V\left[X_{1}, \ldots, X_{r}, X, Y\right]$ and

$$
\widetilde{X}=\operatorname{Proj}(R[U, V] / X V=Y U)
$$

and let $U_{1} \subset \tilde{X}$ be the open set

$$
\operatorname{Spec}(R[u] / X=Y u) \simeq \operatorname{Spec}\left(V\left[X_{1}, \ldots, X_{r}, Y, u\right]\right)
$$

and let $U_{2}$ be the other open set

$$
U_{2}=\operatorname{Spec}(R[v] / X v=Y) \simeq \operatorname{Spec}\left(V\left[X_{1}, \ldots, X_{r}, X, v\right]\right) .
$$

Let $R_{i}$ denote the coordinate ring of $U_{i}$ and let $R_{12}$ denote the coordinate ring of the intersection $U_{1} \cap U_{2}$. Let $R_{\infty}, R_{i \infty}$ and $R_{12 \infty}$ be the rings obtained by taking all the $p^{n}$-th power roots of the variables $(n \geq 1)$, and let $J_{i, \infty} \subset R_{i, \infty}$ be the ideal defined by the roots of the variable $X$ on $U_{2}$ and the variables $Y$ and $u$ on $U_{1}$ (the inverse image of $D_{1}$ ). Also let $J_{\infty} \subset R_{\infty}$ be the ideal defined by the roots of $X$. The ideals $J_{i, \infty}$ define the same ideal in $R_{12, \infty}$ which we denote simply by $J_{12, \infty}$. Also define $I_{i, \infty} \subset R_{i, \infty}$ to be the unit ideal on $U_{2}$ and the ideal defined by the $p^{n}$-th roots of $u$ on $U_{1}$ (the strict transform of $D_{1}$ ). The ideals $I_{i}$ define the same ideal in $R_{12, \infty}$ which we denote by $I_{12, \infty}$ (the unit ideal).

Note that the automorphism groups of $U_{i, \infty}^{o}\left(\right.$ resp. $\left.U_{12, \infty}^{o}, \operatorname{Spec}\left(R_{\infty}\right)^{o}\right)$ over $U_{i}^{o}$ (resp. $\left.U_{12}^{o}, \operatorname{Spec}(R)^{o}\right)$ are all isomorphic via the natural maps. Call this group $\Delta$. Then the cohomology of $\mathscr{J}_{E}$ is given by $H^{*}\left(\Delta, J_{\infty}\right)$, the cohomology of $\mathscr{J}_{E_{1}}$ is given by the cohomology $H^{*}\left(\Delta, J_{1, \infty} \oplus J_{2, \infty} \rightarrow J_{12, \infty}\right)$, and the cohomology of $\mathscr{J}_{E_{2}}$ is given by $H^{*}\left(\Delta, I_{1, \infty} \oplus I_{2, \infty} \rightarrow I_{12, \infty}\right)$. Then the lemma follows from observing that the natural maps

$$
J_{\infty} \rightarrow\left(J_{1, \infty} \oplus J_{2, \infty} \rightarrow J_{12, \infty}\right)
$$


and

$$
J_{\infty} \rightarrow\left(I_{1, \infty} \oplus I_{2, \infty} \rightarrow I_{12, \infty}\right)
$$

are quasi-isomorphism which is an immediate verification.

8.57. The analogous result also holds for étale cohomology. Consider again the commutative diagram

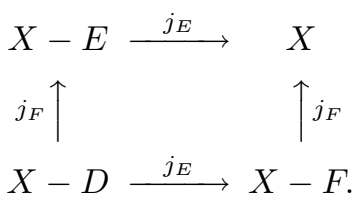

Let $L$ be a locally constant constructible sheaf on $X-D$. Set $M=R j_{E *} j_{F !} L$. On $\widetilde{X}$ we can then also consider $M_{1}=R j_{E_{1} *} j_{F_{1}} ! \pi^{*} L$ and $M_{2}=R j_{E_{2} *} j_{F_{2}} ! \pi^{*} L$. We then have maps

$$
\pi^{*} M \rightarrow M_{2} \leftarrow M_{1}
$$

Proposition 8.58. The induced maps

$$
M \rightarrow R \pi_{*} M_{2} \leftarrow R \pi_{*} M_{1}
$$

are isomorphisms.

Proof. By the proper base change theorem [1, XII.5.1] it suffices to show that for any geometric point $\bar{x} \rightarrow X$ with $e: N_{\bar{x}}=\widetilde{X} \times_{X} \bar{x} \rightarrow \widetilde{X}$ the fiber, the maps

$$
M_{\bar{x}} \rightarrow H^{*}\left(N_{\bar{x}}, e^{*} M_{2}\right) \leftarrow H^{*}\left(N_{\bar{x}}, e^{*} M_{1}\right)
$$

are isomorphisms. This is clear if $\bar{x}$ has image in the complement of $D_{1} \cap D_{2}$ so it suffices to consider the case when $\bar{x}$ has image in $D_{1} \cap D_{2}$. In this case $N_{\bar{x}}$ is isomorphic to $\mathbb{P}^{1}, M_{\bar{x}}=0$, and $e^{*} M_{2}$ is also trivial. Thus the only issue is $e^{*} M_{1}$.

The pair $(X, D)$ is étale locally isomorphic to $X_{1} \times \mathbb{A}^{2}$ together with a divisor $D^{\prime} \subset X_{1}$ such that $D_{1}=X_{1} \times \mathbb{A}^{1} \times\{0\}, D_{2}=X_{1} \times\{0\} \times \mathbb{A}^{1}$, and $D=D_{1} \cup$ $D_{2} \cup D^{\prime} \times \mathbb{A}^{2}$. Furthermore, étale locally on $X$ the sheaf $L$ is isomorphic to the sheaf associated to a representation of $\widehat{\mathbb{Z}}(1)^{r}$ (the Galois group of the extension of $X$ obtained by taking roots of the components of the divisor $D$ ). By considering the distinguished triangles obtained from filtering $L$ it suffices to consider the case when $L$ is a simple sheaf, and hence of the form $L_{\mu}$ for some character $\mu$ of $\widehat{\mathbb{Z}}(1)^{d}$ (notation as in 3.5). Furthermore, by the projection formula one reduces to the case when $L$ is obtained by pullback via the second projection $X_{1} \times \mathbb{G}_{m}^{2} \rightarrow \mathbb{G}_{m}^{2}$ from a rank 1 sheaf on $\mathbb{G}_{m}^{2}$. By the proper base change theorem this reduces the proof to the case when $X=\mathbb{P}^{1} \times \mathbb{P}^{1}$ with divisor $D=\left\{0 \times \mathbb{P}^{1}, \mathbb{P}^{1} \times 0, \infty \times \mathbb{P}^{1}, \mathbb{P}^{1} \times \infty\right\}$ and $D_{1}=\infty \times \mathbb{P}^{1}$ and $D_{2}=\mathbb{P}^{1} \times \infty$.

With these assumptions, consider first the case of the constant sheaf $\Lambda$. The exceptional fiber $e: N \hookrightarrow \widetilde{X}$ is equal to $\mathbb{P}^{1}$ and we can compute the pullback $e^{*} j_{E_{1} !} R j_{F_{1} *} \Lambda$ explicitly. Indeed let $X^{\prime}$ denote $X-E_{1}$ and consider the diagram

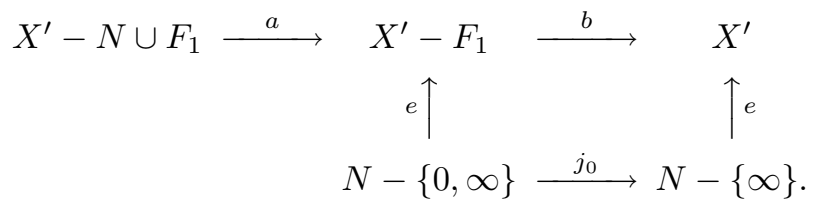


Then $e^{*} j_{E_{1}}$ ! $R j_{F_{1} *} \Lambda$ is isomorphic to the extension by zero along $N-\infty \hookrightarrow N$ of $e^{*} R b_{*} R a_{*} \Lambda$. By the proper base change theorem we have $e^{*} R b_{*} R a_{*} \Lambda \simeq R j_{0 *} e^{*} R a_{*} \Lambda$. By cohomological purity [1, XVI.3.9] there is a distinguished triangle on $X^{\prime}-F_{1}$

$$
\cdots \rightarrow \Lambda \rightarrow R a_{*} \Lambda \rightarrow e_{*} \Lambda(-1)[-1] \rightarrow \cdots
$$

which upon applying $e^{*}$ gives a distinguished triangle on $N-\{0, \infty\}$

$$
\cdots \rightarrow \Lambda \rightarrow e^{*} R a_{*} \Lambda \rightarrow \Lambda(-1)[-1] \rightarrow \cdots
$$

Applying $j_{\infty}$ ! and $R j_{0 *}$ we obtain a distinguished triangle on $N$

$$
\cdots \rightarrow j_{\infty} ! R j_{0 *} \Lambda \rightarrow e^{*} j_{E_{1} !} R j_{F_{1} *} \Lambda \rightarrow j_{\infty} ! R j_{0 *} \Lambda(-1)[-1] \rightarrow \cdots,
$$

where $j_{0}$ and $j_{\infty}$ are the inclusions of the complements of the points $0, \infty \in \mathbb{P}^{1}$ respectively. Thus it suffices to show that $H_{c}^{*}\left(\mathbb{A}^{1}, R j_{0 *} \Lambda\right)$ is zero. For this consider again the distinguished triangle on $\mathbb{A}^{1}$ (where $i:\{0\} \hookrightarrow \mathbb{A}^{1}$ is the inclusion)

$$
\Lambda \rightarrow R j_{0 *} \Lambda \rightarrow i_{*} \Lambda(-1)[-1] \rightarrow \Lambda[1] .
$$

Consideration of the associated long exact sequence then gives the result.

For the case of a general sheaf $L$, we show that $H^{i}\left(N_{\bar{x}}, e^{*} M_{1}\right)$ is zero by induction on $i$. For the base case we take $i=-1$ where the result is vacuous.

For the inductive step, fix $i$ and assume that $H^{j}\left(N_{\bar{x}}, e^{*} M_{1}\right)=0$ for $j<i$ and any locally constant constructible sheaf $L$ on $X-D$. We then show that $H^{i}\left(N_{\bar{x}}, e^{*} M_{1}\right)=0$. By the same argument used above it suffices to consider the case when $L$ has rank 1. Let $N$ be an integer such that the pullback of $L$ under the map $\rho: \mathbb{G}_{m}^{2} \rightarrow \mathbb{G}_{m}^{2}$ given by multiplication by $N$ on each factor is trivial. Then we get an inclusion

$$
L \hookrightarrow \rho_{*} \Lambda
$$

giving a short exact sequence

$$
0 \rightarrow L \rightarrow \rho_{*} \Lambda \rightarrow Q \rightarrow 0
$$

This short exact sequence induces a distinguished triangle

$$
R j_{E_{1} *} j_{F_{1}} ! \pi^{*} L \rightarrow R j_{E_{1} *} j_{F_{1}} ! \pi^{*} \rho_{*} \Lambda \rightarrow R j_{E_{1} *} j_{F_{1}} ! \pi^{*} Q \rightarrow R j_{E_{1} *} j_{F_{1}} ! \pi^{*} L[1]
$$

and hence an exact sequence

$H^{i-1}\left(N_{\bar{x}}, e^{*} R j_{E_{1} *} j_{F_{1}} ! \pi^{*} Q\right) \rightarrow H^{i}\left(N_{\bar{x}}, e^{*} R j_{E_{1} *} j_{F_{1}} ! \pi^{*} L\right) \rightarrow H^{i}\left(N_{\bar{x}}, e^{*} R j_{E_{1} *} j_{F_{1}} ! \pi^{*} \rho_{*} \Lambda\right)$.

Since $H^{i-1}\left(N_{\bar{x}}, e^{*} R j_{E_{1} *} j_{F_{1}} ! \pi^{*} Q\right)=0$ by the induction hypothesis it therefore suffices to consider $L=\rho_{*} \Lambda$.

Let $\rho: \mathbb{P}^{1} \times \mathbb{P}^{1} \rightarrow \mathbb{P}^{1} \times \mathbb{P}^{1}$ also denote the map raising the coordinates to the $N$-th power. Then there is a commutative diagram

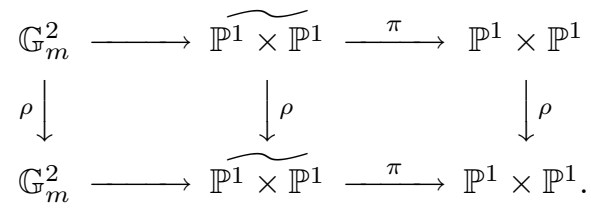

Using this one we are then reduced to the case of the constant sheaf $\Lambda$ which was already shown. 
8.59. We apply all this to complete the proof of 6.16 as follows. Let $(X, D)$ be as in 8.2 Let $Y$ be the blow-up of $X \times X$ along the intersections $D_{i} \times D_{i}$ of the divisors $D_{i} \times X$ and $X \times D_{i}$. Then the diagonal map $X \rightarrow X \times X$ induces a canonical morphism $i: X \hookrightarrow Y$ such that $D$ meets the boundary divisor $D_{Y}$ of $Y$ (the inverse image of $D \times X \cup X \times D$ ) transversally. Let $\pi: Y \rightarrow X \times X$ be the projection. Let $D_{Y}=E_{1} \cup F_{1}$ be the decomposition obtained by taking $E_{1}$ equal to the union of the strict transforms of the divisors $X \times D_{i}$, and let $D_{Y}=E_{2} \cup F_{2}$ be the decomposition obtained by taking $E_{2}$ equal to the union of the exceptional divisors with the strict transforms of the $X \times D_{i}$. Then cup product for étale cohomology is given by the composite

$$
\begin{aligned}
R \Gamma\left(X_{\bar{K}}^{o}, L\right) \otimes R \Gamma_{c}\left(X_{\bar{K}}^{o}, L^{*}\right) & \simeq R \Gamma_{E_{1}, F_{1}}\left(Y_{\bar{K}}^{o}, L \otimes L^{*}\right) \\
& \stackrel{\sim}{\simeq} \Gamma_{E_{2}, F_{2}}\left(Y_{\bar{K}}^{o}, L \otimes L^{*}\right) \\
& R \Gamma_{c}\left(X_{\bar{K}}^{o}, \operatorname{End}(L)\right),
\end{aligned}
$$

where $L \otimes L^{*}$ denotes the pullback to $Y$ of the sheaf $\operatorname{pr}_{1}^{*} L \otimes \operatorname{pr}_{2}^{*} L^{*}$ on $X \times X$. In other words, let $\delta_{\text {et }} \in H_{F_{2}, E_{2}}^{2 d}\left(Y_{K}^{o}, L^{*} \otimes L\right)(d)$ denote the class $i_{*}(1)$, where $1 \in$ $H^{0}\left(X_{\bar{K}}^{o}\right.$, End $\left.(L)\right)$ is the class corresponding to the identity map $L \rightarrow L$. Then the Poincaré duality pairing

$$
R \Gamma\left(X_{\bar{K}}^{o}, L\right) \otimes R \Gamma_{c}\left(X_{\bar{K}}^{o}, L^{*}\right) \rightarrow \mathbb{Z} / p^{r}(-d)[-2 d]
$$

is given by the composite

$$
\begin{array}{rlc}
R \Gamma\left(X_{\bar{K}}^{o}, L\right) \otimes R \Gamma_{c}\left(X_{\bar{K}}^{o}, L^{*}\right) & \simeq & R \Gamma_{E_{1}, F_{1}}\left(Y_{K}^{o}, L \otimes L^{*}\right) \\
& \stackrel{\sim}{\simeq} \Gamma_{E_{2}, F_{2}}\left(Y_{K}^{o}, L \otimes L^{*}\right) \\
& \stackrel{\cup \text { et }}{\longrightarrow} & R \Gamma_{c}\left(Y_{K}^{o}, \mathbb{Z} /\left(p^{r}\right)\right)(d)[2 d] \\
& \stackrel{\mathbb{t r}}{\longrightarrow} & \mathbb{Z} / p^{r}(-d)[-2 d] .
\end{array}
$$

Similarly, the Poincaré duality pairing

$$
R \Gamma\left(\mathscr{X}_{\bar{K}}^{o}, L \otimes \mathscr{O}_{\mathscr{X}_{K}^{o}}\right) \otimes R \Gamma\left(\mathscr{X}_{\bar{K}}^{o}, L^{*} \otimes \mathscr{J}_{X}\right) \rightarrow \bar{V} /\left(p^{r}\right)(-d)[-2 d]
$$

is given by the composite

$$
\begin{aligned}
& R \Gamma\left(\mathscr{X}_{\frac{o}{K}}^{o}, L \otimes \mathscr{O}_{\mathscr{X}_{\frac{o}{K}}}\right) \otimes R \Gamma\left(\mathscr{X}_{\frac{o}{K}}^{o}, L^{*} \otimes \mathscr{J}_{X}\right) \simeq R \Gamma\left(\mathscr{Y}_{K}^{o}, L \otimes L^{*} \otimes \mathscr{J}_{E_{1}}\right) \\
& \underset{\sim}{\simeq} R \Gamma\left(\mathscr{Y} \frac{o}{K}, L \otimes L^{*} \otimes \mathscr{J}_{E_{2}}\right) \\
& \underset{\operatorname{tr}}{\stackrel{\cup}{\longrightarrow}} \quad R \Gamma\left(\mathscr{\mathscr { Y }} \frac{o}{K}, \mathscr{J}_{\mathscr{Y}} \frac{o}{K}\right)(d)[2 d] \\
& \stackrel{\operatorname{tr}}{\rightarrow} \quad \bar{V} / p^{r}(-d)[-2 d]
\end{aligned}
$$

where $\delta \in H^{2 d}\left(\mathscr{Y} \frac{o}{K}, L^{*} \otimes L \otimes \mathscr{J}_{F_{2}}\right)(d)$ is the class $i_{*}(1)$. If $\sigma: R \Gamma_{F_{2}, E_{2}}\left(Y_{\bar{K}}, \mathbb{Z} / p^{r}\right) \rightarrow$ $R \Gamma\left(\mathscr{Y}_{\bar{K}}, \mathscr{J}_{F_{2}}\right)$ is the transformation 8.19 .2 we have by 8.21 that $\sigma\left(\delta_{\mathrm{et}}\right)=\delta$. Also there is a similar description of the Poincaré duality pairings for cohomology with partial compact support. 
Now since the trace map in either theory has the property that it sends the cohomology class of a point in the interior to 1, the diagram

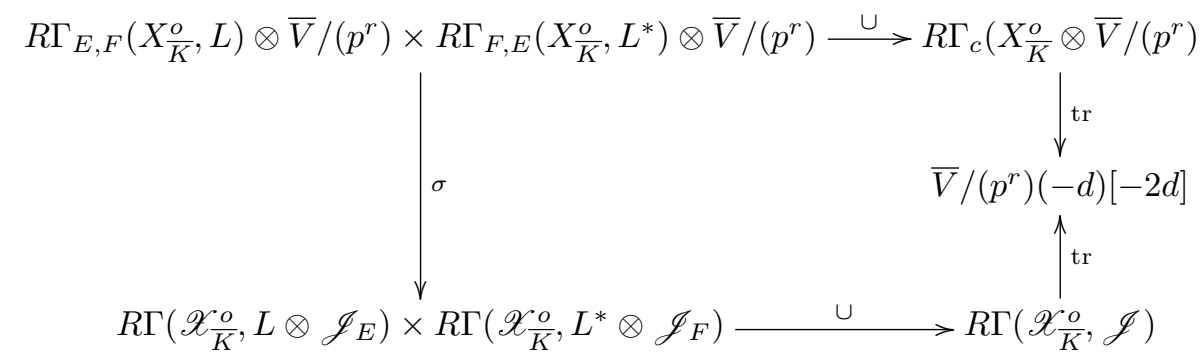

commutes (somewhat abusively we say that $\sigma$ is an "isometry").

Let $\gamma: R \Gamma\left(\mathscr{X}_{\bar{K}}^{o}, L \otimes \mathscr{J}_{E}\right) \rightarrow R \Gamma_{E, F}\left(X_{\bar{K}}^{o}, L\right) \otimes \bar{V} / p^{r}$ denote the adjoint of $\sigma$.

Lemma 8.60. The diagram $(8.60 .1)$

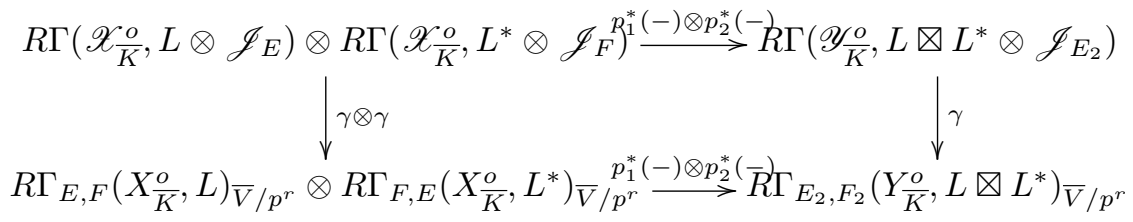

commutes.

Proof. Let $\rho_{1}$ (resp. $\rho_{2}$ ) denote the composite morphism

$$
\rho_{1}:=\left(p_{1}^{*}(-) \otimes p_{2}^{*}(-)\right) \circ(\gamma \otimes \gamma) \quad\left(\operatorname{resp} . \quad \rho_{2}:=\gamma \circ\left(p_{1}^{*}(-) \otimes p_{2}^{*}(-)\right)\right) .
$$

Let $\tau_{i}$ denote the composite morphism

$$
\begin{gathered}
\left(R \Gamma\left(\mathscr{X}_{\bar{K}}^{o}, L \otimes \mathscr{J}_{E}\right) \otimes R \Gamma\left(\mathscr{X}_{\bar{K}}^{o}, L^{*} \otimes \mathscr{J}_{F}\right)\right) \otimes\left(R \Gamma_{F, E}\left(X_{\bar{K}}^{o}, L^{*}\right) \otimes R \Gamma_{E, F}\left(X_{\bar{K}}^{o}, L\right)\right) \\
\downarrow \rho_{i} \otimes\left(p_{1}^{*}(-) \otimes p_{2}^{*}(-)\right) \\
R \Gamma_{E_{2}, F_{2}}\left(Y_{\bar{K}}^{o}, L \otimes L^{*}\right)_{\bar{V} / p^{r}} \otimes R \Gamma_{F_{2}, E_{2}}\left(Y_{\bar{K}}^{o}, L^{*} \otimes L\right) \\
\downarrow \\
\quad \text { Poincaré duality } \\
\bar{V} / p^{r}(-2 d)[-4 d] .
\end{gathered}
$$

By duality, to show that $\rho_{1}=\rho_{2}$ it suffices to show that $\tau_{1}=\tau_{2}$. This follows from the definition of $\gamma$, which implies that both $\tau_{1}$ and $\tau_{2}$ are equal to the composite

$$
\begin{aligned}
& \left(R \Gamma\left(\mathscr{X}_{\bar{K}}^{o}, L \otimes \mathscr{J}_{E}\right) \otimes R \Gamma\left(\mathscr{X}_{\bar{K}}^{o}, L^{*} \otimes \mathscr{J}_{F}\right)\right) \otimes\left(R \Gamma_{F, E}\left(X_{\bar{K}}^{o}, L^{*}\right) \otimes R \Gamma_{E, F}\left(X_{\bar{K}}^{o}, L\right)\right) \\
& \downarrow \operatorname{id} \otimes \mathrm{id} \otimes \sigma \otimes \sigma \\
& R \Gamma\left(\mathscr{X}_{\bar{K}}^{o}, L \otimes \mathscr{J}_{E}\right) \otimes R \Gamma\left(\mathscr{X}_{\bar{K}}^{o}, L^{*} \otimes \mathscr{J}_{F}\right) \otimes R \Gamma\left(\mathscr{X}_{\bar{K}}^{o}, L^{*} \otimes \mathscr{J}_{F}\right) \otimes R \Gamma\left(\mathscr{X}_{\bar{K}}^{o}, L \otimes \mathscr{J}_{E}\right) \\
& \downarrow(\alpha, \beta, \gamma, \delta) \mapsto\langle\alpha, \gamma\rangle \cdot\langle\beta, \delta\rangle \\
& \bar{V} / p^{r}(-2 d)[-4 d],
\end{aligned}
$$

where the last map is the map induced from the Poincaré duality pairings

$$
R \Gamma\left(\mathscr{X}_{\bar{K}}^{o}, L \otimes \mathscr{J}_{E}\right) \otimes R \Gamma\left(\mathscr{X}_{\bar{K}}^{o}, L^{*} \otimes \mathscr{J}_{F}\right) \rightarrow \bar{V} / p^{r}(-d)[-2 d],
$$


and

$$
R \Gamma\left(\mathscr{X}_{\bar{K}}^{o}, L^{*} \otimes \mathscr{J}_{F}\right) \otimes R \Gamma\left(\mathscr{X}_{K}^{o}, L \otimes \mathscr{J}_{E}\right) \rightarrow \bar{V} / p^{r}(-d)[-2 d] .
$$

8.61. It follows that the diagram

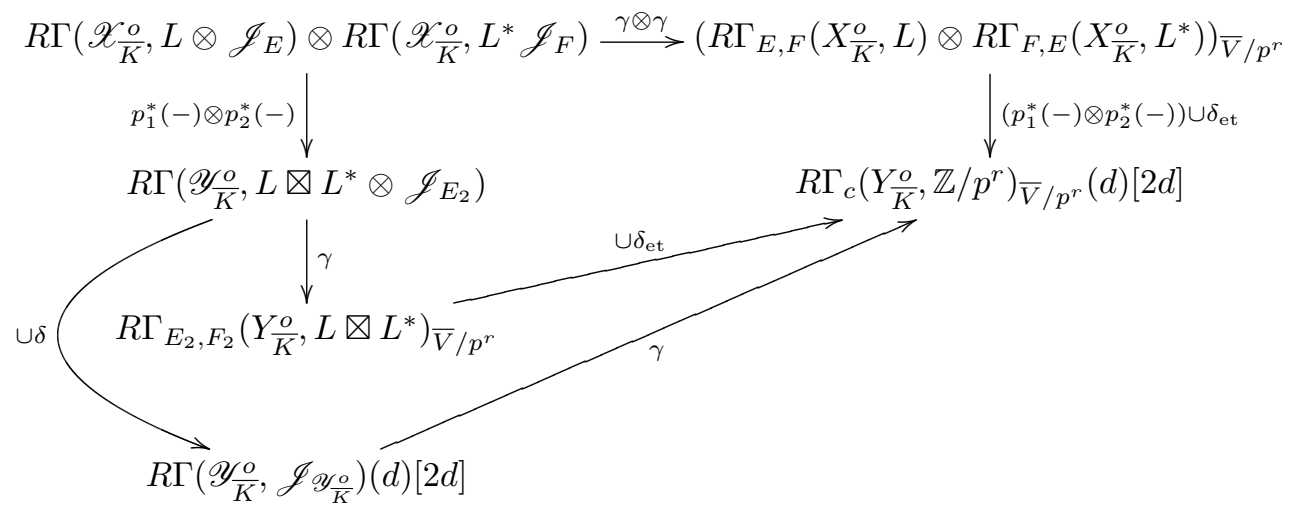

commutes, which implies that the diagram

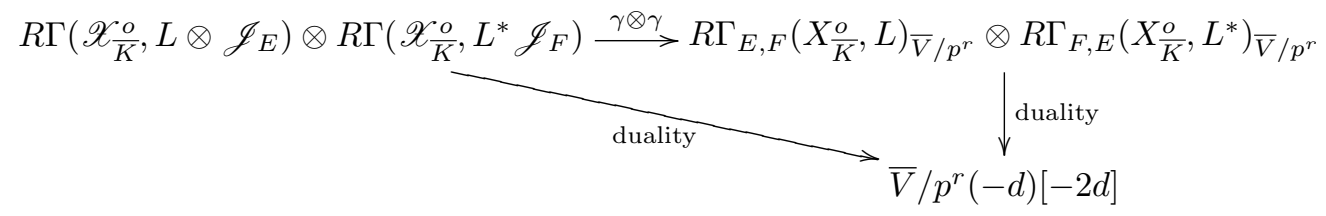

commutes ( $\gamma$ is an isometry).

Since $\gamma \circ \sigma$ is the identity, it follows that both $\sigma$ and $\gamma$ are isomorphisms. This completes the proof of 6.16 .

\section{The topos $\widehat{\mathscr{X}_{\bar{K}}^{o}}$}

9.1. Let $(X, D)$ be as in 6.1 and assume in addition that $X$ is proper over $V$. Let $\widehat{X}$ denote the formal scheme over $\operatorname{Spf}(V)$ obtained by taking the $p$-adic completion of $X$ and define another site $\widehat{\mathscr{S}}$ as follows. The objects of $\widehat{\mathscr{S}}$ are pairs $(U, W)$ where $U \rightarrow \widehat{X}$ is an étale morphism of formal schemes with $U$ affine, and $W \rightarrow \operatorname{Spec}\left(\Gamma\left(U, \mathscr{O}_{U}\right) \otimes_{V} \bar{K}\right)^{o}$ is a finite étale morphism of schemes. Morphisms $\left(U^{\prime}, W^{\prime}\right) \rightarrow(U, W)$ in $\widehat{\mathscr{S}}$ are commutative diagrams

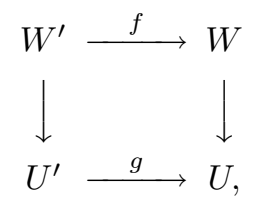

where $g$ is an $\widehat{X}$-morphism. A collection of morphisms $\left\{\left(U_{i}, W_{i}\right) \rightarrow(U, W)\right\}$ is a covering if the collections $\left\{U_{i} \rightarrow U\right\}$ and $\left\{W_{i} \rightarrow W\right\}$ are étale coverings in the usual sense. We denote by $\widehat{\mathscr{X}_{\bar{K}}^{o}}$ the associated topos. There is a sheaf of rings $\mathscr{O}_{\widehat{\mathscr{X}_{K}}}$ in $\widehat{\mathscr{X}_{\bar{K}}} \mathrm{o}$ given by

$$
(U, W) \mapsto \Gamma\left(\bar{W}, \mathscr{O}_{\bar{W}}\right),
$$


where $\bar{W}$ denotes the normalization of $\operatorname{Spec}\left(\Gamma\left(U, \mathscr{O}_{U}\right)\right)$ in $W$. More generally, for any decomposition $D=E \cup F$ of the boundary we have a sheaf of ideals $\mathscr{J}_{\widehat{E}} \subset \mathscr{O}_{\widehat{\mathscr{K}_{K}}}$ which to any $(U, W)$ associates the ideal of the fiber product $\left(\bar{W} \times \operatorname{Spec}\left(\Gamma\left(U, \mathscr{O}_{U}\right)\right)\right.$ $\widehat{E})_{\text {red }} \subset \bar{W}$, where $\widehat{E} \subset \widehat{X}$ denotes the $p$-adic completion of $E$.

9.2. There is a natural morphism of topoi

$$
p: \widehat{\mathscr{X}_{\bar{K}}^{o}} \rightarrow \mathscr{X}_{\bar{K}}^{o}
$$

induced from the morphism of sites $\mathscr{S} \rightarrow \widehat{\mathscr{S}}$ sending a pair $(U, W)$, with $U$ affine, to the $p$-adic completion $\widehat{U}$ with the morphism $W \times_{U} \operatorname{Spec}\left(\Gamma\left(\widehat{U}, \mathscr{O}_{\widehat{U}}\right) \otimes_{V} \bar{K}\right)^{o} \rightarrow$ $\operatorname{Spec}\left(\Gamma\left(\widehat{U}, \mathscr{O}_{\widehat{U}}\right) \otimes_{V} \bar{K}\right)^{o}$. For any decomposition $D=E \cup F$ there is a natural morphism $p^{*} \mathscr{J}_{E} \rightarrow \mathscr{J}_{\widehat{E}}$ inducing a morphism

$$
\mathscr{J}_{E} \rightarrow R p_{*} \mathscr{J}_{\widehat{E}}
$$

Note also that the map $U \mapsto\left(U, \operatorname{Spec}\left(\Gamma\left(U, \mathscr{O}_{U}\right)\right) \frac{o}{K}\right)$ induces a morphism of sites $\operatorname{Et}(\widehat{X}) \rightarrow \widehat{\mathscr{S}}$ inducing a morphism of topoi $\nu_{\widehat{X}}: \widehat{\mathscr{X}}_{\bar{K}}^{o} \rightarrow \widehat{X}_{\text {et }}$ sitting in a commutative diagram

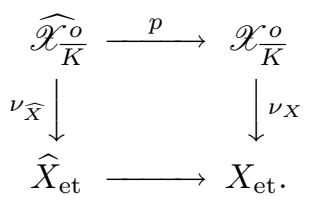

For any affine $U=\operatorname{Spf}(R) \in \operatorname{Et}(\widehat{X})$ there is also a canonical morphism of topoi

$$
\epsilon_{U}: \widehat{\mathscr{X}_{\bar{K}}^{o}} \rightarrow \operatorname{Spec}(R \otimes \bar{K})_{\mathrm{Fet}}^{o}
$$

induced by the morphism of sites sending $W \rightarrow \operatorname{Spec}(R \otimes \bar{K})^{o}$ to $(U, W) \in \widehat{\mathscr{S}}$.

Proposition 9.3. Let $L$ be a locally constant sheaf of $\mathbb{Z} /\left(p^{r}\right)$-modules on $X_{\bar{K}}^{o}$,et' and let $\mathscr{L}$ (resp. $\widehat{\mathscr{L}}$ ) denote the induced sheaf on $\mathscr{X}_{\bar{K}}^{o}$ (resp. $\left.\widehat{\mathscr{X}_{\bar{K}}}\right)$. Then the natural map

$$
p^{*} R \nu_{X *} \mathscr{L} \otimes \mathscr{J}_{E} \rightarrow R \nu_{\widehat{X} *} \widehat{\mathscr{L}} \otimes \mathscr{J}_{\widehat{E}}
$$

is an almost isomorphism and for every $i$ the sheaves

$$
R^{i} \nu_{X *}\left(\mathscr{L} \otimes \mathscr{J}_{E}\right) \text { and } R^{i} \nu_{\widehat{X} *} \widehat{\mathscr{L}} \otimes \mathscr{J}_{\widehat{E}}
$$

are almost isomorphic to coherent sheaves.

Proof. We may work étale locally on $X$ so can assume that $X=\operatorname{Spec}(R)$ and that there is a formally étale morphism $\operatorname{Spec}(R) \rightarrow \operatorname{Spec}\left(V\left[X_{1}, \ldots, X_{d}\right]\right)$ as in 2.16.1. Let $\widehat{R}$ denote the $p$-adic completion of $X, R_{\infty}$ the extension of $R$ obtained by taking all the $p^{n}$-th roots of the variables $X_{i}$, and let $\widehat{R}_{\infty}$ denote the $p$-adic completion of $R_{\infty} \otimes_{R} \widehat{R}$. Let $J_{\infty} \subset R_{\infty}$ denote the ideal generated by the roots of the element $\prod_{i \in E} X_{i}$. Let $\Delta_{\infty} \simeq \mathbb{Z}_{p}(1)^{d}$ denote the Galois group of $R_{\infty}$ over $R$, which is also the Galois group of $\widehat{R}_{\infty}$ over $\widehat{R}$. Then by 3.10 we may assume that $L$ is obtained from a representation of $\Delta_{\infty}$ and then the morphism 9.3.1 is identified in $\widetilde{D}(\widehat{R})$ with the morphism of complexes of quasi-coherent sheaves associated to the morphism of complexes of $\widehat{R}$-modules

$$
R \Gamma_{\Delta_{\infty}}\left(L \otimes J_{\infty}\right) \otimes_{R} \widehat{R} \rightarrow R \Gamma_{\Delta_{\infty}}\left(L \otimes J_{\infty} \otimes_{R_{\infty}} \widehat{R}_{\infty}\right) .
$$


The proposition therefore follows from 3.12 .

COROLlary 9.4. For any locally constant sheaf of $\mathbb{Z} /\left(p^{r}\right)$-modules $L$ in $\mathscr{X}_{\bar{K}}^{\circ}$ the natural map

$$
H^{*}\left(\mathscr{X}_{\bar{K}}^{o}, \mathscr{L} \otimes \mathscr{J}_{E}\right) \rightarrow H^{*}\left(\widehat{\mathscr{X}_{K}^{o}}, p^{*} \mathscr{L} \otimes \mathscr{J}_{\widehat{E}}\right)
$$

is an almost isomorphism.

Proof. Combine 9.3 with Grothendieck's comparison theorem [11, III.5.1.2].

\section{An aside on Galois cohomology}

In order to compare étale cohomology to crystalline cohomology it is necessary to compute global cohomology using group cohomology. This is a standard application of simplicial techniques so we just sketch the necessary details here.

Let $\Delta$ denote the standard simplicial category of finite order sets with order preserving morphisms.

10.1. Let us first recall the standard complex representing group cohomology. Let $X$ be a connected normal scheme and let $\eta: \operatorname{Spec}(\Omega) \rightarrow X$ be a geometric point mapping to the generic point. Denote by $\Delta$ the fundamental group of $X$ with respect to the base point $\eta$. Then the topos $X_{\mathrm{Fet}}$ is equivalent to the category of (discrete) sets with continuous action of the group $\Delta$. It follows that for an abelian sheaf in $X_{\mathrm{Fet}}$ the cohomology groups $H^{*}\left(X_{\mathrm{Fet}}, L\right)$, or better the complex $R \Gamma(L)$, can be computed as follows.

Write also $L$ for the continuous $\Delta$-representation corresponding to the sheaf $L$. For an integer $n$ let $\mathcal{C}^{n}(\Delta, L)$ denote the group

$$
\mathcal{C}^{n}(\Delta, L):=\left\{\text { continuous } \Delta \text {-equivariant maps } \Delta^{n+1} \rightarrow L\right\},
$$

where $\Delta$ acts on $\Delta^{n+1}$ via the diagonal action on the left. For any morphism $\delta:[n] \rightarrow[m]$ there is an induced map

$$
\Delta^{m+1}=\operatorname{Hom}([m], \Delta) \stackrel{\delta^{*}}{\rightarrow} \operatorname{Hom}([n], \Delta)=\Delta^{n+1}
$$

which induces a map $\delta^{*}: \mathcal{C}^{n}(\Delta, L) \rightarrow \mathcal{C}^{m}(\Delta, L)$. In this way $[n] \mapsto \mathcal{C}^{n}(\Delta, L)$ becomes a cosimplicial abelian group and we define $\mathcal{C}^{\bullet}(\Delta, L)$ to be the total complex of this cosimplicial group. It is well-known that this complex $\mathcal{C}^{\bullet}(\Delta, L)$ computes the group cohomology of $L$ (see for example [44, 2.2]).

If $L^{\bullet}$ is a complex of $\Delta$-modules, then we can apply the $\mathcal{C}^{\bullet}(\Delta,-)$ to each $L^{i}$ to obtain a bicomplex. We denote by $\mathcal{C}^{\bullet}\left(\Delta, L^{\bullet}\right)$ the resulting total complex. Note that there is a canonical map of complexes $L^{\Delta} \rightarrow \mathcal{C}^{\bullet}\left(\Delta, L^{\bullet}\right)$.

10.2. The advantage of this construction is that it is functorial. Let $f: Y \rightarrow X$ be a morphism of connected normal schemes and $\epsilon: \operatorname{Spec}(\Omega) \rightarrow Y$ a lifting of $\eta$ to $Y$. If $\Delta_{Y}$ (resp. $\Delta_{X}$ ) denotes the fundamental group of $Y$ (resp. $X$ ) with respect to the given geometric points, then there is an induced morphism $\Delta_{Y} \rightarrow \Delta_{X}$ of topological groups. This morphism induces a map

$$
f^{*}: \mathcal{C}^{n}\left(\Delta_{X}, L\right) \rightarrow \mathcal{C}^{n}\left(\Delta_{Y}, L\right)
$$

compatible with the cosimplicial structure. The resulting map of complexes $f^{*}$ : $\mathcal{C}^{\bullet}\left(\Delta_{X}, L\right) \rightarrow \mathcal{C}^{\bullet}\left(\Delta_{Y}, L\right)$ represents the pullback morphism on cohomology. 
10.3. More generally, let $X=X_{1} \amalg \cdots \amalg X_{r}$ be a disjoint union of connected normal schemes and assume that for each $i$ we are given a geometric generic point $\eta_{i}: \operatorname{Spec}(\Omega) \rightarrow X_{i}$. We then define $\mathcal{C}^{\bullet}(\Delta, L)$ to be the product of the complexes $\mathcal{C} \bullet\left(\Delta_{X_{i}}, L\right)$. This is again functorial with respect to morphisms of pointed schemes.

A convenient way to deal with non-connected schemes is as follows. If $X$ is a disjoint union of normal schemes as above define a base point for $X$ to be a disjoint union of geometric points $E=\coprod_{i=1}^{r} \operatorname{Spec}(\Omega)$ with a morphism of schemes $E \rightarrow X$ such that for each connected component $X_{i}$ of $X$ there exists a unique point of $E$ mapping to the generic point of $X_{i}$. Then we can define $\mathcal{C}^{\bullet}(\Delta, L)$ using $E$ and this complex is functorial with respect to commutative diagrams

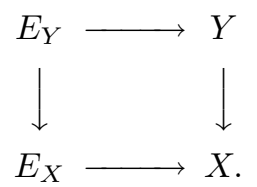

In particular, if $U$. is a simplicial scheme such that each $U_{n}$ is a disjoint union of normal schemes and $E$. $\rightarrow U$. is a morphism of simplicial schemes such that for every $n$ the morphism $E_{n} \rightarrow U_{n}$ is a base point, then we obtain a cosimplicial complex $\mathcal{C}^{\bullet}(U ., L)$.

If $X=\coprod X_{i}$ is a disjoint union of normal schemes as above and $E \rightarrow X$ is a base point, we define a Galois module on $X$ to be the data of a continuous representation of each $\pi_{1}\left(X_{i}\right)$ (with respect to the base point given by $E$ ). For such a module $L$ we can then define $\mathcal{C}^{\bullet}(\Delta, L)$

10.4. The main example we will consider is the following. Let $X / V$ be a smooth proper geometrically connected $V$-scheme and $D \subset X$ a divisor with relative normal crossings. As usual we write $X^{o}$ for $X-D$ and for any $X$-scheme $U$ we write $U^{o}$ for $U \times_{X} X^{o}$. Fix a geometric point $\operatorname{Spec}(\Omega) \rightarrow X$ mapping to the generic point of $X$. Let $U$. $\rightarrow X$ be a smooth hypercover by affine schemes such that for every $n$ the scheme $U_{n}$ is a disjoint union of open subschemes of $X$ and such that each $U_{n, \bar{K}}^{o}$ is a $K(\pi, 1)$. Let $\pi_{0}\left(U_{n}\right)$ denote the set of connected components of $U_{n, \bar{K}}$. The set of connected components is functorial so we obtain a simplicial set $\pi_{0}(U$.), and since each connected component of $U_{n}$ is an open subset there is a canonical base point $\operatorname{Spec}(\Omega)^{\pi_{0}(U .)} \rightarrow U$.. Thus for a sheaf $L$ on $U_{\text {.,Fet }}^{o}$ we obtain a complex $\mathcal{C}^{\bullet}(U ., L)$. We write $G C(U ., L)$ for the associated simple complex. If $L$ is obtained from a locally constant constructible sheaf on $X_{\bar{K}}^{o}$ then since each $U_{n, \bar{K}}^{o}$ is a $K(\pi, 1)$ this complex computes $H^{*}\left(X_{\bar{K}, \text { et }}^{o}, L\right)$.

10.5. With $U$. as in the previous paragraph, we will also need to consider the $p$ adic completion $U^{\wedge}$ of $U$. The scheme $U_{n}^{\wedge}$ is the spectrum of the $p$-adic completion of the coordinate ring of $U_{n}$. Let $\widehat{\Omega}$ be the $p$-adic completion of the field $\Omega$. Then for any connected component $P$ of $U_{n}^{\wedge}$ there exists a unique morphism $\operatorname{Spec}(\widehat{\Omega}) \rightarrow P$ such that the diagram

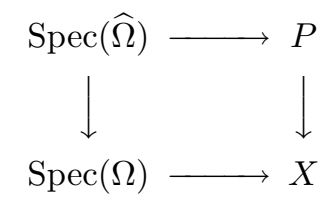


commutes. We therefore also obtain a base point $E$. $\rightarrow U^{\wedge}$. For a sheaf $L$ on $U_{\text {.,Fet }}^{\wedge}$ we can then define the complex $G C\left(U^{\wedge}, L\right)$ using this base point.

In what follows, we usually omit the initial choice of base point from the discussion. The careful reader should make the straightforward verification that all the results are independent of the choice of the geometric generic point of $X$.

REMARK 10.6. When dealing with algebraic spaces or Deligne-Mumford stacks, one must take extra care in dealing with the choice of base point as one cannot work Zariski topology and instead must work with the étale topology. The necessary technicalities for dealing with this problem are discussed in [41, §4].

\section{Fontaine's big rings}

11.1. Let $R$ be a $\bar{V}$-algebra which is an integral domain. Choose an algebraic closure $\operatorname{Frac}(R) \hookrightarrow \Omega$ and assume that Frobenius on $R / p R$ is surjective and that $\operatorname{Spec}(R / p R)$ is connected and nonempty. Then Fontaine's theory gives rings $\mathrm{A}_{\text {cris }}(R)$ and $\mathrm{B}_{\text {cris }}(R)$ defined as follows. First set

$$
S_{R}:=\lim _{\longleftarrow} R / p R
$$

where the projective limit is taken with respect to the Frobenius morphism on $R / p R$ ( $S_{R}$ is the perfection of $R / p R$ ). Since $S_{R}$ is perfect, the ring of Witt vectors $W\left(S_{R}\right)$ has a canonical lift of Frobenius. An element $x \in W\left(S_{R}\right)$ can be represented by a vector $\left(x_{0}, x_{1}, x_{2}, \ldots\right)$ where each $x_{i}=\left(x_{i 0}, x_{i 1}, \ldots\right)$ is an infinite vector with $x_{i j} \in R / p R$ and such that $x_{i(j+1)}^{p}=x_{i j}$. There is a natural map

$$
\theta: W\left(S_{R}\right) \longrightarrow \widehat{R}
$$

defined by sending $x$ as above to

$$
\theta(x)=\underset{m}{\lim }\left(\tilde{x}_{0 m}^{p^{m}}+p \tilde{x}_{1 m}^{p^{m-1}}+\cdots+p^{m} \tilde{x}_{m m}\right),
$$

where $\tilde{x}_{i j} \in \widehat{R}$ is any lift of $x_{i j}$. Here $\widehat{R}$ denotes the $p$-adic completion of $R$. The assumption that Frobenius on $R / p R$ is surjective ensures that the map $\theta$ is surjective [49, A1.1].

For $n \geq 0$ we write $W_{n}\left(S_{R}\right)$ for the ring of Witt vector of length $n+1$.

We set $J=\operatorname{Ker}(\theta)$ and define $\mathrm{A}_{\text {cris }}(R)$ to be the $p$-adic completion of the divided power envelope $D_{J}\left(W\left(S_{R}\right)\right)$. We thus obtain a diagram

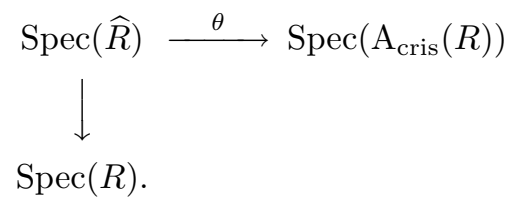

Let Fil $\mathrm{A}_{\text {cris }}(R)$ denote the filtration on $\mathrm{A}_{\text {cris }}(R)$ obtained as the $p$-adic completion of the filtration on $D_{J}\left(W\left(S_{R}\right)\right)$ defined by the PD-ideals $J^{[r]} \subset D_{J}\left(W\left(S_{R}\right)\right)$. For an element $\alpha \in \operatorname{Fil}^{1} \mathrm{~A}_{\text {cris }}(R)$ and an integer $r \geq 1$ we write $\alpha^{[r]} \in \operatorname{Fil}^{r} \mathrm{~A}_{\text {cris }}(R)$ for the element obtained from the $r$-th divided power operation on $J^{[1]}$.

Choose elements $\epsilon_{m} \in \widehat{R}$ with $\epsilon_{0}=1, \epsilon_{m+1}^{p}=\epsilon_{m}$, and $\epsilon_{1} \neq 1$. Let $\epsilon \in S_{R}$ denote the element obtained from the reductions of the $\epsilon_{i}$, and let $[\epsilon] \in W\left(S_{R}\right)$ be 
the Teichmuller lift of $\epsilon$. Set $\pi_{\epsilon}:=[\epsilon]-1 \in W\left(S_{R}\right)$. Then $\pi_{\epsilon} \in \mathrm{Fil}^{1} \mathrm{~A}_{\text {cris }}(R)$ so we can define

$$
t=\log ([\epsilon])=\sum_{m \geq 1}(-1)^{m-1}(m-1) ! \pi_{\epsilon}^{[m]} \in \mathrm{A}_{\text {cris }}(R) .
$$

Define

$$
\mathrm{B}_{\text {cris }}(R)^{+}:=\mathrm{A}_{\text {cris }}(R) \otimes \mathbb{Q}
$$

and

$$
\mathrm{B}_{\text {cris }}(R):=\mathrm{B}_{\text {cris }}(R)^{+}\left[\frac{1}{t}\right] .
$$

In fact, by [49, A3.2] we have $t^{p-1} \in p \mathrm{~A}_{\text {cris }}(R)$ and therefore $\mathrm{B}_{\text {cris }}(R)=\mathrm{A}_{\text {cris }}(R)[1 / t]$. The element $t \in \mathrm{A}_{\text {cris }}(R)$ lies in $\operatorname{Fil}^{1} \mathrm{~A}_{\text {cris }}(R)$ and we obtain a filtration $\operatorname{Fil}_{\mathrm{B}_{\text {cris }}(R)}$ on $\mathrm{B}_{\text {cris }}(R)$ by declaring that $1 / t$ has degree -1 .

More canonically, we can describe this localization as follows. The group $\mathbb{Z}_{p}(1)$ is by definition the group of sequences $\left(\zeta_{n}\right)_{n \geq 0}$, where $\zeta_{n}$ is a $p^{n}$-th root of 1 and $\zeta_{n+1}^{p}=\zeta_{n}$. The above construction then induces a homomorphism

$$
\alpha: \mathbb{Z}_{p}(1) \rightarrow \mathrm{A}_{\text {cris }}(R)^{*}
$$

whose image is contained in the elements mapping to 1 in $\widehat{R}$. Taking the logarithm of this map we obtain a map

$$
\beta: \mathbb{Q}_{p}(1) \rightarrow \mathrm{B}_{\text {cris }}(R)
$$

whose image is the line spanned by $t$. This map induces for every $i$ an isomorphism

$$
\beta^{\otimes i}: \mathrm{B}_{\text {cris }}(R)(i) \rightarrow \mathrm{B}_{\text {cris }}(R) .
$$

11.2. The ring $\mathrm{A}_{\text {cris }}(R)$ has a lift of Frobenius $\varphi_{\mathrm{A}_{\text {cris }}(R)}$ induced by the canonical lift of Frobenius to $W\left(S_{R}\right)$. This lifting of Frobenius induces a semi-linear automorphism $\varphi_{\mathrm{B}_{\text {cris }}(R)}$ of $\mathrm{B}_{\text {cris }}(R)$ and preserves the filtration $\operatorname{Fil}_{\mathrm{B}_{\text {cris }}(R)}$.

Also define a second filtration $I^{[\cdot]}$ on $\mathrm{A}_{\text {cris }}(R)$ by

$$
I^{[r]}:=\left\{x \in \mathrm{A}_{\text {cris }}(R) \mid \varphi^{n}(x) \in \mathrm{Fil}^{r} \mathrm{~A}_{\text {cris }}(R) \text { for all } n \geq 0\right\} .
$$

Lemma 11.3. Let $S$ be a p-adically complete and separated ring flat over $\mathbb{Z}_{p}$. Let $\xi \in S$ be an element whose image in $S / p S$ is a regular element. Then

(i) $\xi$ is a regular element of $S$.

(ii) The sequence

$$
0 \longrightarrow S\langle u\rangle \stackrel{\xi-u}{\longrightarrow} S\langle u\rangle \stackrel{u \mapsto \xi}{\longrightarrow} S \otimes \mathbb{Q}
$$

is exact where $S\langle u\rangle$ denotes the free $P D$-polynomial algebra on one variable.

Proof. For (i) suppose $\beta \in S$ is an element with $\xi \beta=0$. We show by induction on $n$ that $\beta$ is in $p^{n} S$ for all $n$. This implies that $\beta=0$ since $\cap_{n \geq 1} p^{n} S=0$. So assume that $\beta \in p^{n} S$ and write $\beta=p^{n} \beta^{\prime}$. Then $\xi \beta^{\prime}=0$ since $S$ is flat over $\mathbb{Z}_{p}$ and $p^{n} \xi \beta^{\prime}=\xi \beta=0$. Since $\xi$ maps to a regular element in $S / p S$ it follows that $\beta^{\prime} \in p S$ and therefore $\beta \in p^{n+1} S$.

For (ii) note that the injectivity of multiplication by $\xi-u$ on $S\langle u\rangle$ follows from (i): If $f=\sum_{i \geq i_{0}} a_{i} u^{[i]}$ is an element with $a_{i_{0}} \neq 0$ then

$$
(\xi-u) f=a_{i_{0}} \xi u^{\left[i_{0}\right]}+\sum_{i>i_{0}} b_{i} u^{[i]}
$$


for some $b_{i} \in S$. Since $\xi a_{i_{0}} \neq 0$ by (i) it follows that $(\xi-u) f \neq 0$.

For exactness in the middle of 11.3.1 suppose $f=\sum_{i} a_{i} u^{[i]}$ maps to zero in $S \otimes \mathbb{Q}$. We show by induction on the smallest integer $n$ for which $f$ can be written as

$$
f=a_{i_{0}} u^{\left[i_{0}\right]}+\cdots+a_{i_{0}+n} u^{\left[i_{0}+n\right]}
$$

that $f$ is in the image of $(\xi-u)$. So consider $f$ mapping to zero in $S \otimes \mathbb{Q}$ and write $f$ in the form 11.3.3. Since $f$ maps to zero in $S \otimes \mathbb{Q}$ we have

$$
a_{i_{0}} \frac{\xi^{i_{0}}}{i_{0} !}+\cdots+a_{i_{0}+n} \frac{\xi^{i_{0}+n}}{\left(i_{0}+n\right) !}=0
$$

in $S \otimes \mathbb{Q}$, and since $S$ is flat over $\mathbb{Z}_{p}$ this implies that

$$
\frac{\left(i_{0}+n\right) !}{i_{0} !} a_{i_{0}} \xi^{i_{0}}+\cdots+a_{i_{0}+n} \xi^{i_{0}+n}=0
$$

in $S$ (note that the coefficients in this expression are integers). Since $\xi$ is a regular element in $S$ this implies that

$$
\frac{\left(i_{0}+n\right) !}{i_{0} !} a_{i_{0}}=\xi \beta
$$

for some $\beta \in S$. Furthermore, since $\xi$ maps to a regular element in $S / p S$ it follows that $\beta=\frac{\left(i_{0}+n\right) !}{i_{0} !} \beta^{\prime}$ for some $\beta^{\prime} \in S$ and hence by the flatness of $S$ over $\mathbb{Z}_{p}$ we have $a_{i_{0}}=\xi \beta^{\prime}$ for some $\beta^{\prime} \in S$. Subtracting $(\xi-u) \beta^{\prime} u^{\left[i_{0}\right]}$ from $f$ we are then reduced to the case when $f$ can be written in the form

$$
f=a_{i_{0}} u^{\left[i_{0}+1\right]}+\cdots+a_{i_{0}+n-1} u^{\left[i_{0}+1+n-1\right]}
$$

which by induction on $n$ completes the proof of (ii).

11.4. Choose $\nu_{m} \in R$ such that $\nu_{0}=-p$ and $\nu_{m+1}^{p}=\nu_{m}$ (note that this is possible since $R$ is a $\bar{V}$-algebra). Let $-p \in S_{R}$ denote the element defined by the reductions $\nu_{m}(\bmod p)$. Let $[-p] \in \bar{W}\left(S_{R}\right)$ denote the Teichmuller lifting of $\underline{-p}$, and let $\xi:=[\underline{-p}]+p$. By [49, A2.2] the element $\xi$ generates the kernel of $\theta: W\left(S_{R}\right) \rightarrow \widehat{R}$ and $\xi$ satisfies the assumptions of 11.3 . In particular the sequence

$$
0 \longrightarrow W\left(S_{R}\right)\langle u\rangle \stackrel{\xi-u}{\longrightarrow} W\left(S_{R}\right)\langle u\rangle \stackrel{\tau}{\longrightarrow} W\left(S_{R}\right) \otimes \mathbb{Q}
$$

is exact, where $\tau$ is the map sending $u^{[i]}$ to $\xi^{i} / i$ !.

As before let $D_{J}\left(W\left(S_{R}\right)\right)$ denote the divided power envelope of the surjection $W\left(S_{R}\right) \rightarrow \widehat{R}$. By [49, A2.8] there exists a unique inclusion $D_{J}\left(W\left(S_{R}\right)\right) \hookrightarrow$ $W\left(S_{R}\right) \otimes \mathbb{Q}$ whose composite with $W\left(S_{R}\right) \rightarrow D_{J}\left(W\left(S_{R}\right)\right)$ is the inclusion $W\left(S_{R}\right) \hookrightarrow$ $W\left(S_{R}\right) \otimes \mathbb{Q}$, and via this inclusion $D_{J}\left(W\left(S_{R}\right)\right)$ is identified with the image of $\tau$. It follows from this that $D_{J}\left(W\left(S_{R}\right)\right)$ is $p$-torsion free, and that for every $n$ there is an exact sequence

$0 \longrightarrow W\left(S_{R}\right) / p^{n}\langle u\rangle \stackrel{\xi-u}{\longrightarrow} W\left(S_{R}\right) / p^{n}\langle u\rangle \stackrel{\tau}{\longrightarrow} D_{J}\left(W\left(S_{R}\right)\right) / p^{n} \longrightarrow 0$. 
Also for all integers $r<n$ we obtain a commutative diagram with exact rows (11.4.3)

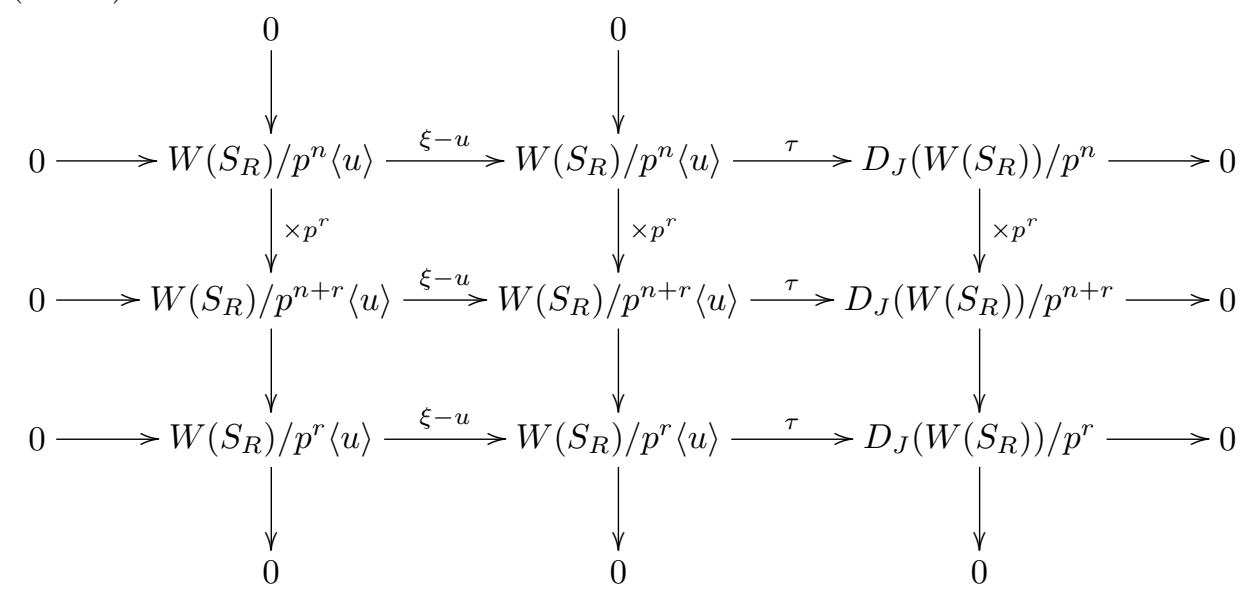

By the snake lemma and the fact that the first two columns are exact we conclude that the sequence

$$
0 \longrightarrow D_{J}\left(W\left(S_{R}\right)\right) / p^{n} \stackrel{\times p^{r}}{\longrightarrow} D_{J}\left(W\left(S_{R}\right)\right) / p^{n+r} \longrightarrow D_{J}\left(W\left(S_{R}\right)\right) / p^{r} \longrightarrow 0
$$

is also exact. As $n$ varies the short exact sequences 11.4 .4 form an exact sequence of projective systems and passing to the limit we obtain an exact sequence

$$
0 \longrightarrow \mathrm{A}_{\text {cris }}(R) \stackrel{\times p^{r}}{\longrightarrow} \mathrm{A}_{\text {cris }}(R) \longrightarrow D_{J}\left(W\left(S_{R}\right)\right) / p^{r} \longrightarrow 0 .
$$

In particular, we can rewrite 11.4 .2 as

$0 \longrightarrow W\left(S_{R}\right) / p^{n}\langle u\rangle \stackrel{\xi-u}{\longrightarrow} W\left(S_{R}\right) / p^{n}\langle u\rangle \stackrel{\tau}{\longrightarrow} \mathrm{A}_{\text {cris }}(R) / p^{n} \longrightarrow 0$.

Lemma 11.5. For any integer $n$ the natural map

$$
W_{n}\left(S_{R}\right) \otimes_{W_{n}\left(S_{\bar{V}}\right)} \mathrm{A}_{\text {cris }}(\bar{V}) \rightarrow \mathrm{A}_{\text {cris }}(R) / p^{n+1}
$$

is an isomorphism. 
Proof. This follows from the exactness of 11.4.6 and consideration of the commutative diagram

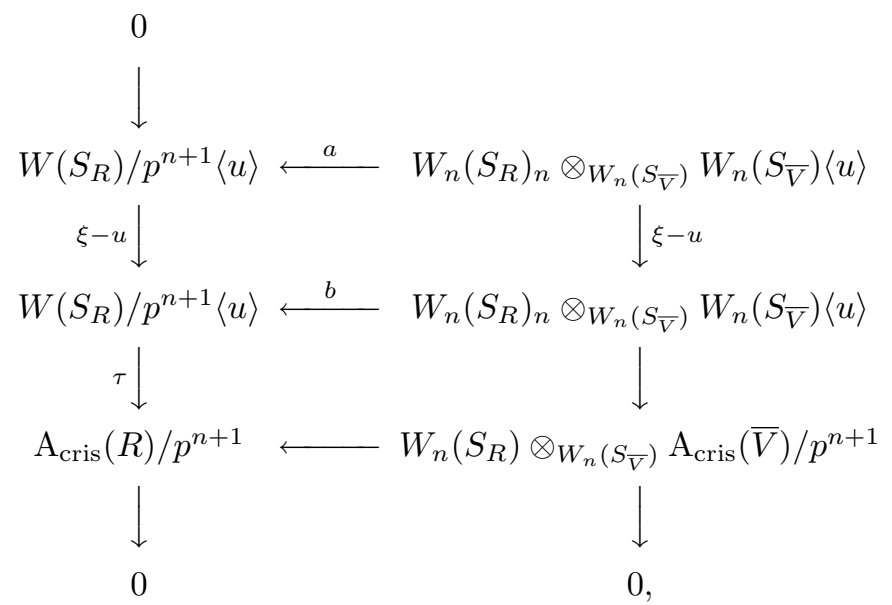

where the maps labelled $a$ and $b$ are clearly isomorphisms.

Lemma 11.6. For any integer $n \geq 0$ the map

$$
R^{\wedge} \rightarrow \operatorname{gr}_{\text {Fil }_{\mathrm{A}_{\text {cris }}}^{n}} \mathrm{~A}_{\text {cris }}\left(R^{\wedge}\right), f \mapsto f \cdot \xi^{[n]}
$$

is an isomorphism. In particular, the natural map

$$
\operatorname{gr}_{\mathrm{Fil}_{\mathrm{A}_{\text {cris }}}} \mathrm{A}_{\text {cris }}(\bar{V}) \otimes_{\bar{V}^{\wedge}} R^{\wedge} \rightarrow \operatorname{gr}_{\mathrm{Fil}_{\mathrm{A}_{\text {cris }}}} \mathrm{A}_{\text {cris }}\left(R^{\wedge}\right)
$$

is an isomorphism.

Proof. This is shown in [49, A2.11].

11.7. Since the image of $\xi$ in $\mathrm{A}_{\text {cris }}(\bar{V})$ is in the divided power ideal of $\mathrm{A}_{\text {cris }}(\bar{V})$, the map

$$
W\left(S_{\bar{V}}\right) /(p) \rightarrow \mathrm{A}_{\text {cris }}(\bar{V}) / p
$$

factors through $W\left(S_{\bar{V}}\right) /\left(p, \xi^{p}\right)$ since $\xi^{p}=p ! \xi^{[p]}=0$ in $\mathrm{A}_{\text {cris }}(\bar{V}) / p$.

Lemma 11.8. Let $i \in \mathbb{N}$ be an integer. For any integer $1 \leq j \leq p$ the map

$$
\times \xi^{[i p]}: W\left(S_{\bar{V}}\right) /\left(p, \xi^{j}\right) \rightarrow J^{[i p]} /\left(p, J^{[i p+j]}\right)
$$

is an isomorphism, where $J=\operatorname{Ker}\left(\theta: \mathrm{A}_{\text {cris }}(\bar{V}) \rightarrow \bar{V}^{\wedge}\right)$.

Proof. The case $j=1$ follows from [49, A2.9]. The general case follows by induction and consideration of the diagrams (11.8.2)

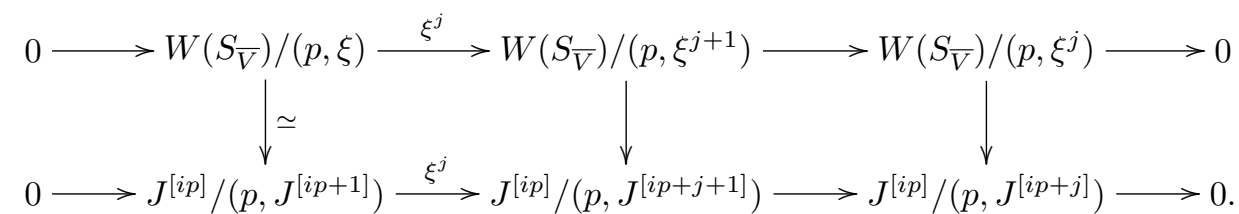


Here the top row is exact since $\xi$ maps to a regular element in $W\left(S_{\bar{V}}\right) /(p)$, and the bottom row is exact since for $j<p$ we have

$$
\xi^{[i p+j]}=\frac{(i p) !}{(i p+j) !} \xi^{j} \cdot \xi^{[i p]}
$$

and $(i p) ! /(i p+j)$ ! has $p$-adic valuation 0 .

Lemma 11.9. The map of $W\left(S_{\bar{V}}\right) /\left(p, \xi^{p}\right)$-modules

$$
\oplus_{i \geq 0} W\left(S_{\bar{V}}\right) /\left(p, \xi^{p}\right) \stackrel{\oplus \xi^{[i p]}}{\longrightarrow} \mathrm{A}_{\text {cris }}(\bar{V}) / p
$$

is an isomorphism. In particular, $\mathrm{A}_{\text {cris }}(\bar{V}) / p$ is flat over $W\left(S_{\bar{V}}\right) /\left(p, \xi^{p}\right)$.

Proof. The map is clearly surjective. That it is injective follows from the preceding lemma.

11.10. In what follows we will need to consider "almost mathematics" over the ring $\mathrm{B}_{\text {cris }}(\bar{V})$. Let $\Lambda \subset \mathbb{Q}$ be the subring $\mathbb{Z}[1 / p]$, and let $\Lambda_{+}:=\Lambda \cap \mathbb{Q}_{>0}$. Fix a sequence $\left(\tau_{m}\right)_{m \geq 0}$ of elements of $\bar{V}$ with $\tau_{0}=p$ and $\tau_{m+1}^{p}=\tau_{m}$ for all $m \geq 0$. We define $\tau_{m}:=0$ for $m<0$. Then for any $n \in \mathbb{Z}$ define $\lambda_{1 / p^{n}}$ to be the element $\left(a_{m}\right)_{m \geq 0} \in S_{\bar{V}}$ with

$$
a_{m}=\tau_{m+n} .
$$

Note that $\lambda_{1 / p^{n}}^{p}=\lambda_{1 / p^{n-1}}$.

Lemma 11.11. For every $m \in \mathbb{Z}$ the map

$$
\times \lambda_{1 / p^{n}}: S_{\bar{V}} \rightarrow S_{\bar{V}}
$$

is injective.

Proof. Let $F: S_{\bar{V}} \rightarrow S_{\bar{V}}$ be the Frobenius morphism (which is a bijection). Then $F\left(\lambda_{1 / p^{n}}\right)=\lambda_{1 / p^{n-1}}$ so the diagram

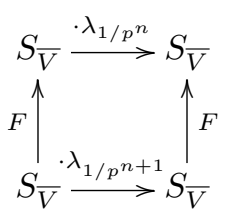

commutes. It follows that multiplication by $\lambda_{1 / p^{n}}$ is injective if and only if multiplication by either $\lambda_{1 / p^{n-1}}$ or $\lambda_{1 / p^{n+1}}$ is injective. It follows that it suffices to consider the case $n=0$. Now with the notation of [49, A2] we have $-\lambda_{1}=\underline{-p}$, and therefore the result in the case $n=0$ follows from [49, A2.1].

11.12. For $n \in \mathbb{Z}$, let $\delta_{1 / p^{n}} \in W\left(S_{\bar{V}}\right)$ denote the Teichmuller lifting $\left[\lambda_{1 / p^{n}}\right]$ of $\lambda_{1 / p^{n}}$. Since the Teichmuller lifting is multiplicative we have

$$
\delta_{1 / p^{n}}^{p}=\sigma\left(\delta_{1 / p^{n}}\right)=\delta_{1 / p^{n-1}},
$$

where $\sigma: W\left(S_{\bar{V}}\right) \rightarrow W\left(S_{\bar{V}}\right)$ is the canonical lifting of Frobenius.

Since $W\left(S_{\bar{V}}\right)$ is $p$-torsion free and $p$-adically separated, it follows from 11.11 that

$$
\times \delta_{1 / p^{n}}: W\left(S_{\bar{V}}\right) \rightarrow W\left(S_{\bar{V}}\right)
$$

is injective. 
For $\alpha \in \Lambda_{+}$, we define $\delta_{\alpha} \in W\left(S_{\bar{V}}\right)$ as follows. Write $\alpha=s / p^{n}$ for some $s \in \mathbb{Z}_{>0}$ and $n \in \mathbb{Z}$, and define

$$
\delta_{\alpha}:=\delta_{1 / p^{n}}^{s}
$$

Note that if $s=p^{r} s^{\prime}$ with $\left(s^{\prime}, p\right)=1$ then

$$
\delta_{1 / p^{n}}^{s}=\left(\delta_{1 / p^{n}}^{p^{r}}\right)^{s^{\prime}}=\delta_{1 / p^{n-r}}^{s^{\prime}},
$$

which implies that this definition of $\delta_{\alpha}$ is independent of how we write $\alpha$ as a fraction $s / p^{n}$.

Similarly we can define $\lambda_{\alpha} \in S_{\bar{V}}$ for any $\alpha \in \Lambda_{+}$. Note that since $\delta_{1 / p^{n}}$ (resp. $\left.\lambda_{1 / p^{n}}\right)$ is not a zero divisor in $W\left(S_{\bar{V}}\right)$ (resp. $S_{\bar{V}}$ ), the element $\delta_{\alpha}$ (resp. $\lambda_{\alpha}$ ) is not a zero divisor in $W\left(S_{\bar{V}}\right)$ (resp. $S_{\bar{V}}$ ) either.

Lemma 11.13. For every $\alpha, \beta \in \Lambda_{+}$we have $\lambda_{\alpha} \cdot \lambda_{\beta}=\lambda_{\alpha+\beta}$ and $\delta_{\alpha} \cdot \delta_{\beta}=\delta_{\alpha+\beta}$.

Proof. Since the Teichmuller lifting is multiplicative, it suffices to prove the statement about the $\lambda$ 's. Write $\alpha=s / p^{m}$ and $\beta=t / p^{m}$ (note that without loss of generality we may assume that the denominators are the same). Then by definition

$$
\lambda_{\alpha}=\lambda_{1 / p^{m}}^{s}, \quad \lambda_{\beta}=\lambda_{1 / p^{m}}^{t}, \quad \text { and } \quad \lambda_{\alpha+\beta}=\lambda_{1 / p^{m}}^{s+t}
$$

which implies the result.

11.14. For $\alpha \in \Lambda_{+}$let $\mathfrak{m}_{\alpha} \subset W\left(S_{\bar{V}}\right)$ denote the ideal generated by $\delta_{\alpha}$, and let $\mathfrak{m}:=\cup_{\alpha \in \Lambda_{+}} \mathfrak{m}_{\alpha}$. We can then apply the almost theory of section 2 for modules over $W\left(S_{\bar{V}}\right)$.

Since $\mathrm{A}_{\text {cris }}(\bar{V})$ is an integral domain, for any $\alpha \in \Lambda_{+}$the element $\delta_{\alpha}$ is also not a zero divisor in $\mathrm{A}_{\text {cris }}(\bar{V})$ and $\mathrm{B}_{\text {cris }}(\bar{V})$.

Thus we can also apply the almost theory of section 2 with $\mathrm{B}_{\text {cris }}(\bar{V})$ and the ideals $\mathfrak{m}_{\text {cris }, \alpha} \subset \mathrm{B}_{\text {cris }}(\bar{V})$ generated by $\delta_{\alpha}$. In what follows, when we consider almost mathematics of modules over the ring $\mathrm{B}_{\text {cris }}(\bar{V})$ (resp. $W\left(S_{\bar{V}}\right)$ ) it will always be with respect to the ideals $\mathfrak{m}_{\text {cris }, \alpha}$ (resp. $\left.\mathfrak{m}_{\alpha}\right)$.

Let $\widetilde{\mathrm{B}}_{\text {cris }}(\bar{V})$ denote $\mathrm{B}_{\text {cris }}(\bar{V})\left[\delta_{\alpha}^{-1}\right]_{\alpha \in \Lambda_{+}}$.

11.15. The action of $G_{K}$ on $\bar{V}$ induces an action of $G_{K}$ on $S_{\bar{V}}, W\left(S_{\bar{V}}\right)$, and $\mathrm{A}_{\text {cris }}(\bar{V})$ by functoriality. Let $\chi: G_{K} \rightarrow \mathbb{Z}_{p}^{*}$ denote the cyclotomic character. Then it follows from the construction that $G_{K}$ acts on $t$ by

$$
g * t=\chi(g) t .
$$

In particular, the action of $G_{K}$ on $\mathrm{A}_{\text {cris }}(\bar{V})$ induces an action on $\mathrm{B}_{\text {cris }}(\bar{V})$. Also the choice of the elements $\tau_{m}$ defines a homomorphism

$$
\rho: G_{K} \rightarrow \mathbb{Z}_{p}(1)=\varliminf_{n}^{\lim } \mu_{p^{n}}
$$

If $g \in G_{K}$ then the image $\rho(g)=\left(\zeta_{n}\right)$ in $\mathbb{Z}_{p}(1)$ is characterized by the equality

$$
g\left(\tau_{n}\right)=\zeta_{n} \tau_{n} .
$$

One verifies immediately from the construction that for $g \in G_{K}$ we have

$$
g * \delta_{1}=\alpha(\rho(g)) \cdot \delta_{1},
$$

where $\alpha$ is the map 11.1.8. In particular, the $G_{K}$-action on $\mathrm{B}_{\text {cris }}(\bar{V})$ induces an action of $G_{K}$ on $\widetilde{\mathrm{B}}_{\text {cris }}(\bar{V})$. 
11.16. We will apply the preceding discussion as follows. Let $X / V$ be a smooth proper scheme and $D \subset X$ a divisor with normal crossings relative to $V$. For any étale morphism $\operatorname{Spec}(R) \rightarrow X$ for which there exists a morphism as in 2.16.1. let $R^{\wedge}$ denote the $p$-adic completion of $R$, and let $\bar{R}^{\wedge}$ denote the integral closure of $R^{\wedge}$ in the maximal subextension of the field of fractions of $R^{\wedge}$ which is unramified over $\operatorname{Spec}\left(R^{\wedge}\right) \times_{X} X_{\bar{K}}^{o}$. We then apply the preceding discussion to $\bar{R} \wedge$, and denote the resulting ring by $\mathrm{A}_{\text {cris }}\left(R^{\wedge}\right)$. Note that there is a natural action of $\operatorname{Gal}\left(\bar{R}^{\wedge} / R^{\wedge}\right)$ on $S:=S_{\bar{R}} \wedge$ which induces an action of $\operatorname{Gal}\left(\bar{R}^{\wedge} / R^{\wedge}\right)$ on $\mathrm{A}_{\text {cris }}\left(R^{\wedge}\right)$ which in turn induces an action $\rho_{\mathrm{B}_{\text {cris }}\left(R^{\wedge}\right)}$ of Galois on $\mathrm{B}_{\text {cris }}\left(R^{\wedge}\right)$. This action is continuous and compatible with the filtration. Furthermore the induced action on the diagram 11.1.4 commutes with the lift of Frobenius.

If $s: \operatorname{Spec}\left(\Omega^{\prime}\right) \rightarrow \operatorname{Spec}(\Omega)$ is a morphism of geometric generic points of $\operatorname{Spec}(R)$, then there is a natural isomorphism

$$
\iota_{s}: s^{*} \mathrm{~B}_{\text {cris }}\left(R^{\wedge}\right) \rightarrow \mathrm{B}_{\text {cris }}\left(R^{\wedge}\right)^{\prime},
$$

where $\mathrm{B}_{\text {cris }}\left(R^{\wedge}\right)^{\prime}$ denotes the $\operatorname{Gal}\left(\bar{R}^{\prime \wedge} / R^{\wedge}\right)$-module obtained by replacing $\Omega$ with $\Omega^{\prime}$ in the above construction.

11.17. There is a natural $\log$ structure $M_{\mathrm{A}_{\text {cris }}\left(R^{\wedge}\right)}$ on $\operatorname{Spec}\left(\mathrm{A}_{\text {cris }}\left(R^{\wedge}\right)\right)$ defined as follows. Choose an étale map as in 2.16, and write $t_{1}, \ldots, t_{s} \in R$ for the images of the $T_{i}(i=1, \ldots, s)$. For each $i$ and $l$, the extension $R[X] /\left(X^{p^{l}}-t_{i}\right)$ is étale over $R\left[1 /\left(p t_{1} \cdots t_{s}\right)\right]$. It follows that for each $i$, we can choose a sequence $\tau_{i, n}$ of elements in $\bar{R} \wedge$ such that $\tau_{i, n}^{p}=\tau_{i, n-1}$ and $\tau_{i, 0}=t_{i}$. Let $\tau_{i} \in S$ denote the corresponding element. We then get a map

$$
\lambda: \mathbb{N}^{s} \rightarrow W(S), \quad e_{i} \mapsto\left[\tau_{i}\right],
$$

where $\left[\tau_{i}\right]$ denotes the Teichmuller lift of $\tau_{i}$. This defines a log structure on $W(S)$ and hence in turn also a $\log$ structure on $\mathrm{A}_{\text {cris }}\left(R^{\wedge}\right)$. Note that the log structure on $\bar{R}^{\wedge}$ induced by this map $\lambda$ composed with $\theta$ is simply the log structure induced by pulling back $M_{R}$ via the map $\operatorname{Spec}\left(\bar{R}^{\wedge}\right) \rightarrow \operatorname{Spec}(R)$.

We show that the above log structure on $\mathrm{A}_{\text {cris }}\left(R^{\wedge}\right)$ is independent of the choices as follows. Consider a second map as in 2.16 giving elements $t_{1}^{\prime}, \ldots, t_{s}^{\prime} \in R$ defining the $\log$ structure, and let $\tau_{i, n}^{\prime}$ be a choice of roots of the $t_{i}^{\prime}$. Then there exists a unique sequence $u_{i, n} \in \bar{R}^{\wedge *}$ such that $u_{i, n}^{p}=u_{i, n-1}$ and such that $\tau_{i, n}=u_{i, n} \tau_{i, n}^{\prime}$. Letting $u_{i}$ denote the corresponding element of $S$, we see that $\left[\tau_{i}\right]=\left[u_{i}\right] \cdot\left[\tau_{i}^{\prime}\right]$ in $\mathrm{A}_{\text {cris }}\left(R^{\wedge}\right)$, and hence we get a canonical isomorphism between the associated log structures.

It follows from the above discussion that the enlargement 11.1.4 has a natural structure of a logarithmic enlargement

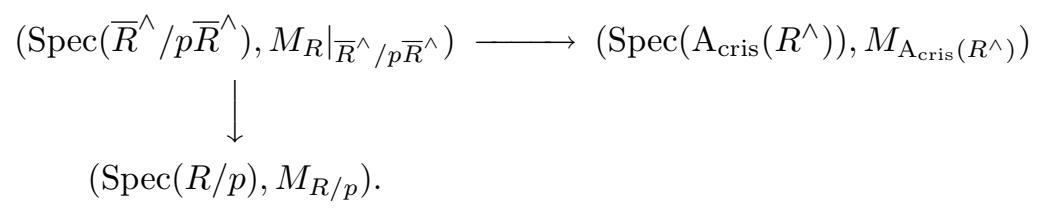

Note also that the action of $\operatorname{Gal}\left(\bar{R}^{\wedge} / R^{\wedge}\right)$ extends naturally to an action on the log scheme $\left(\operatorname{Spec}\left(\mathrm{A}_{\text {cris }}\left(R^{\wedge}\right)\right), M_{\mathrm{A}_{\text {cris }}\left(R^{\wedge}\right)}\right)$. 
11.18. The rings $\mathrm{A}_{\text {cris }}\left(R^{\wedge}\right)$ define a sheaf of rings $\mathscr{A}_{\text {cris }}$ on $\widehat{\mathscr{X}}_{K}^{o}$ as follows. For any object $(U, N) \in \widehat{\mathscr{S}}$ with $U=\operatorname{Spf}\left(R^{\wedge}\right)$ as in 2.16, choose a geometric generic point $\eta: \operatorname{Spec}(\Omega) \rightarrow N$ and set $\mathscr{A}_{\text {cris }}(U, N)$ equal to $\mathrm{A}_{\text {cris }}\left(R^{\wedge}\right)^{\pi_{1}(N, \eta)}$ with the induced filtration. Then this is up to canonical isomorphism independent of the choice of geometric point and $\mathscr{A}_{\text {cris }}$ is defined to be the associated sheaf.

The filtrations $I^{[\cdot]}$ and $\mathrm{Fil}_{\mathrm{A}_{\text {cris }}}$ define two filtrations $\mathscr{I}^{[\cdot]}$ and $\mathrm{Fil} \mathscr{A}_{\text {cris }}$ on $\mathscr{A}_{\text {cris }}$.

11.19. We will also need to consider certain ideals to study cohomology with (partial) compact support. Let $D=E \cup F$ be a decomposition of the boundary so that we have ideals $J_{E} \subset R$ and $\bar{J}_{E} \subset \bar{R}$. Since $\bar{J}_{E} \cap R_{\infty}=J_{E, \infty}$ (notation as in the proof of 8.16 the natural map

$$
J_{E, \infty} \otimes_{R_{\infty}} \bar{R} \rightarrow \bar{J}_{E}
$$

is by 3.4 an almost isomorphism. Since the Frobenius morphism of $R_{\infty} / p R_{\infty}$ clearly induces a surjection on $J_{E, \infty} / p J_{E, \infty}$ this implies that the Frobenius morphism on $\bar{R} / p \bar{R}$ induces an almost surjection on $\bar{J}_{E} / p \bar{J}_{E}$. Also $R_{\infty} / J_{E, \infty}$ is $p$-torsion free, so the sequence

$$
0 \rightarrow \bar{J}_{E} / p \bar{J}_{E} \rightarrow \bar{R} / p \bar{R} \rightarrow \bar{R} /\left(p, \bar{J}_{E}\right) \rightarrow 0
$$

is almost exact. Passing to the inverse limit with respect to the Frobenius morphisms we get by 2.22 an almost exact sequence

$$
0 \rightarrow \lim _{\text {Frob }} \bar{J}_{E} / p \bar{J}_{E} \rightarrow S_{\bar{R} / p \bar{R}} \rightarrow S_{\bar{R} /\left(p, \bar{J}_{E}\right)} \rightarrow 0 .
$$

Define

$$
J_{n, E}^{\inf }:=\operatorname{Ker}\left(W_{n}\left(S_{\bar{R}}\right) \rightarrow W_{n}\left(S_{\bar{R} /\left(p, \bar{J}_{E}\right)}\right)\right)
$$

and

$$
J_{n, E}^{\text {cris }}:=\operatorname{Ker}\left(\mathrm{A}_{\text {cris }}(\bar{R}) / p^{n+1} \rightarrow \mathrm{A}_{\text {cris }}\left(\bar{R} / \bar{J}_{E}\right) / p^{n+1}\right) .
$$

Lemma 11.20. For every $n$ the sequence

$$
0 \rightarrow J_{n, E}^{\mathrm{inf}} \rightarrow W_{n}\left(S_{\bar{R}}\right) \rightarrow W_{n}\left(S_{\bar{R} /\left(p, \bar{J}_{E}\right)}\right) \rightarrow 0
$$

is almost exact.

Proof. The case $n=0$ is 11.19 .3 . The general case follows by induction and consideration of the commutative diagrams

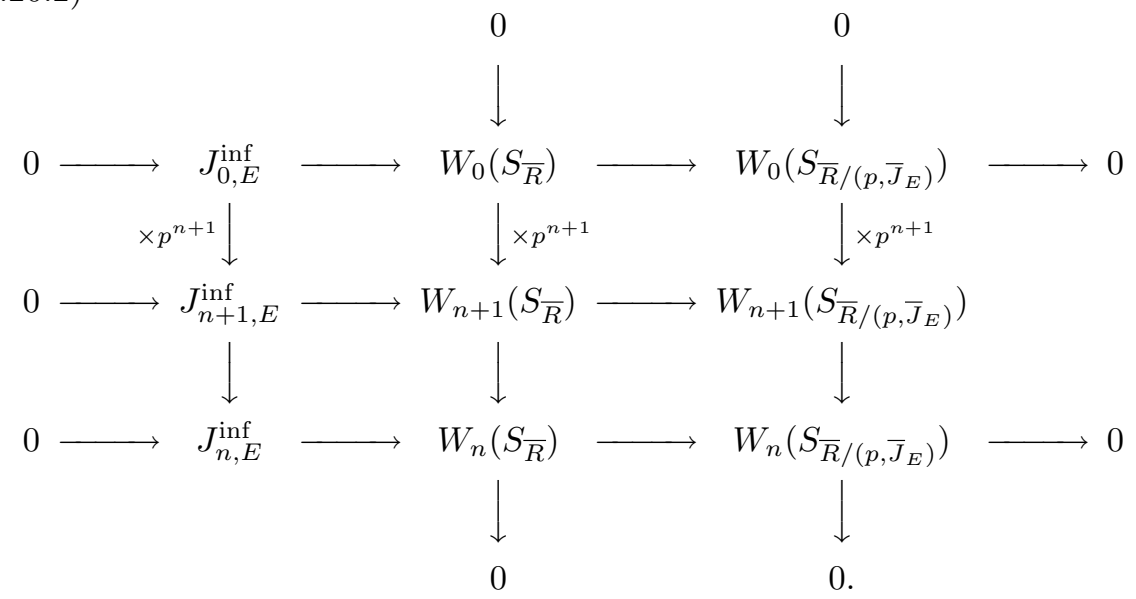


Corollary 11.21. For every integer $n$ the sequence

$$
0 \rightarrow J_{n, E}^{\text {cris }} \rightarrow \mathrm{A}_{\text {cris }}(\bar{R}) / p^{n+1} \rightarrow \mathrm{A}_{\text {cris }}\left(\bar{R} / \bar{J}_{E}\right) / p^{n+1} \rightarrow 0
$$

is almost exact.

Proof. The only issue is the almost surjectivity of the map $\mathrm{A}_{\text {cris }}(\bar{R}) / p^{n+1} \rightarrow$ $\mathrm{A}_{\text {cris }}\left(\bar{R} / \bar{J}_{E}\right) / p^{n+1}$. By 11.5 this map can be identified with the map

$$
\mathrm{A}_{\text {cris }}(\bar{V}) \otimes_{W_{n}\left(S_{\bar{V}}\right)} W_{n}\left(S_{\bar{R}}\right) \rightarrow \mathrm{A}_{\text {cris }}(\bar{V}) \otimes_{W_{n}\left(S_{\bar{V}}\right)} W_{n}\left(S_{\bar{R} / \bar{J}_{E}}\right)
$$

so the almost surjectivity follows from the almost exactness of 11.20 .1

Lemma 11.22. For every integer $n$ the sequence

$$
0 \longrightarrow J_{0, E}^{\mathrm{inf}} \stackrel{\times p^{n}}{\longrightarrow} J_{n, E}^{\mathrm{inf}} \longrightarrow J_{n-1, E}^{\mathrm{inf}} \longrightarrow 0
$$

is almost exact.

Proof. This follows from consideration of the commutative diagram 11.20.2.

LEMma 11.23. For every integer $r \geq 1$ the sequence

$$
0 \rightarrow J_{0, E}^{\inf } / \xi^{r} J_{0, E}^{\inf } \rightarrow S_{\bar{R}} /\left(\xi^{r}\right) \rightarrow S_{\bar{R} / \bar{J}_{E}} /\left(\xi^{r}\right) \rightarrow 0
$$

is almost exact.

Proof. This follows from the almost exactness of 11.20.1 (with $n=0$ ) and the fact that $\xi$ is a regular element in $S_{\bar{R} / \bar{J}_{E}}$.

LEMma 11.24. For every integer $n$ the sequence

$$
0 \rightarrow \mathrm{A}_{\text {cris }}(\bar{V}) \otimes J_{n, E}^{\mathrm{inf}} \rightarrow \mathrm{A}_{\text {cris }}(\bar{V}) \otimes W_{n}\left(S_{\bar{R}}\right) \rightarrow \mathrm{A}_{\text {cris }}(\bar{V}) \otimes W_{n}\left(S_{\bar{R} / \bar{J}_{E}}\right) \rightarrow 0
$$

is almost exact, where the tensor products are taken over $W\left(S_{\bar{V}}\right)$

Proof. Note that for $n=0$ the sequence 11.24.1 can be identified with the sequence obtained by applying $\mathrm{A}_{\text {cris }}(\bar{V}) /(p) \otimes_{S_{\bar{V}} /\left(\xi^{p}\right)}(-)$ to the almost exact sequence

$$
0 \rightarrow J_{0, E}^{\mathrm{inf}} / \xi^{p} J_{0, E}^{\mathrm{inf}} \rightarrow S_{\bar{R}} /\left(\xi^{p}\right) \rightarrow S_{\bar{R} / \bar{J}_{E}} /\left(\xi^{p}\right) \rightarrow 0 .
$$

Since $S_{\bar{V}} /\left(\xi^{p}\right) \rightarrow \mathrm{A}_{\text {cris }}(\bar{V}) / p$ is a flat morphism by 11.9 this proves the case $n=0$. 
The result for general $n$ follows by induction on $n$ and consideration of the diagram (where all tensor products are taken over $W\left(S_{\bar{V}}\right)$ ) $(11.24 .3)$

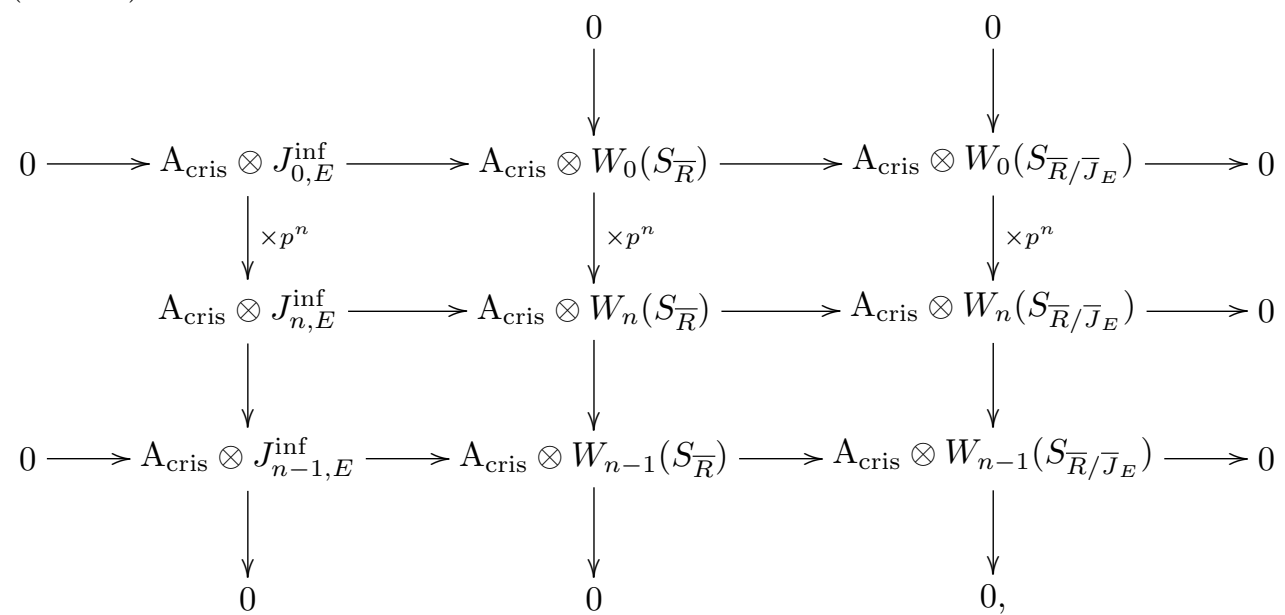

where we write $\mathrm{A}_{\text {cris }}$ for $\mathrm{A}_{\text {cris }}(\bar{V})$, the top and bottom rows are exact by induction and the middle and right columns are exact since they can by 11.5 be identified with the almost exact sequences (note that $\mathrm{A}_{\text {cris }}(\bar{R})$ and $\mathrm{A}_{\text {cris }}\left(\bar{R} / \overline{J_{E}}\right.$ ) are $p$-torsion free)

$$
0 \longrightarrow \mathrm{A}_{\text {cris }}(\bar{R}) / p \stackrel{\times p^{n}}{\longrightarrow} \mathrm{A}_{\text {cris }}(\bar{R}) / p^{n+1} \longrightarrow \mathrm{A}_{\text {cris }}(\bar{R}) / p^{n} \longrightarrow 0
$$

and

$$
0 \longrightarrow \mathrm{A}_{\text {cris }}\left(\bar{R} / \bar{J}_{E}\right) / p \stackrel{\times p^{n}}{\longrightarrow} \mathrm{A}_{\text {cris }}\left(\bar{R} / \bar{J}_{E}\right) / p^{n+1} \longrightarrow \mathrm{A}_{\text {cris }}\left(\bar{R} / \bar{J}_{E}\right) / p^{n} \longrightarrow 0 .
$$

Corollary 11.25. For every integer $n$, the natural map

$$
J_{n, E}^{\mathrm{inf}} \otimes_{W_{n}\left(S_{\bar{V}}\right)} \mathrm{A}_{\text {cris }}(\bar{V}) / p^{n+1} \rightarrow J_{n, E}^{\text {cris }}
$$

is an almost isomorphism. 
Proof. Consider the commutative diagram with almost exact columns (where the tensor products are taken over $\left.W_{n}\left(S_{\bar{V}}\right)\right)$

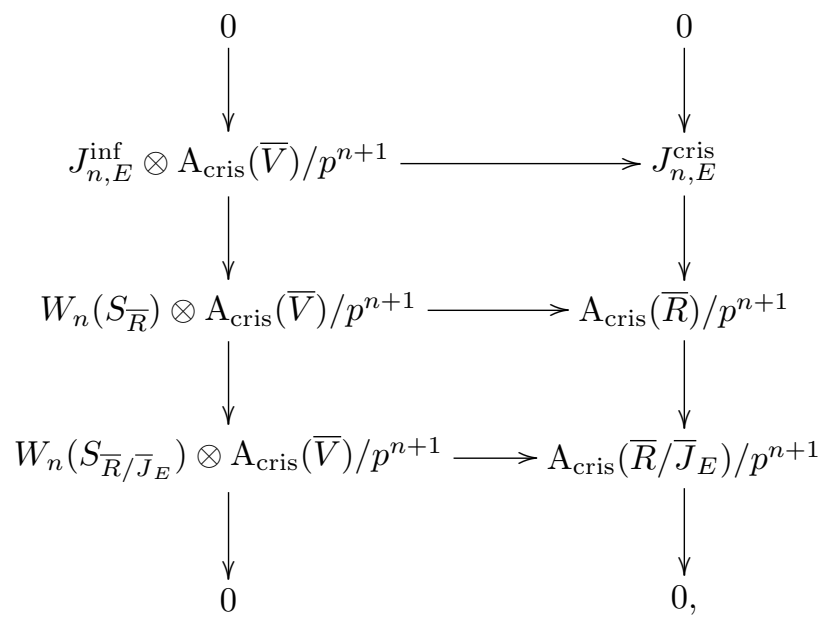

where the right column is almost exact by 11.21 , and the bottom two horizontal arrows are isomorphisms by 11.5 .

\section{More computations of cohomology}

We continue with the notation of 11.16

THEOREM 12.1. Let $L$ be a smooth sheaf of $\mathbb{Z} / p^{n}$-modules on $X_{\bar{K}}^{o}$, let $U$. $\rightarrow X$ be an étale hypercover with each $U_{n}$ admitting a morphism as in 2.16.1 and affine, and let $\widehat{U} . \rightarrow \widehat{X}$ be the p-adic completion of $U$.. Then the transformation

$$
\mathrm{A}_{\text {cris }}(\bar{V}) \otimes_{\mathbb{Z}_{p}} G C\left(U_{\cdot \bar{K}}^{o}, L\right) \rightarrow G C\left(\widehat{U}_{\cdot, \bar{K}}^{o}, L \otimes_{\mathbb{Z}_{p}} \mathrm{~A}_{\text {cris }}(\widehat{U} .)\right)
$$

is an almost isomorphism.

Proof. Consideration of the long exact sequences associated to the short exact sequences

$$
0 \longrightarrow L / p L \stackrel{p^{n}}{\longrightarrow} L / p^{n+1} L \longrightarrow L / p^{n} L \longrightarrow 0
$$

reduces the proof to the case when $n=1$.

Let $S_{\overline{\mathscr{O}}_{\widehat{U}}}$ be the Galois representation which in degree $n$ is given by applying the construction in 11.1 to the coordinate ring of $\widehat{U}_{n}$.

LEMMA 12.2. For any integer $j \geq 1$ the transformation

$$
\left(S_{\bar{V}} /\left(\xi^{j}\right)\right) \otimes_{\mathbb{F}_{p}} G C\left(U_{\cdot, \bar{K}}^{o}, L\right) \rightarrow G C\left(\widehat{U}_{\bar{K}}^{o}, S_{\overline{\mathscr{O}}_{\widehat{U} .}} /\left(\xi^{j}\right) \otimes L\right)
$$

is an almost isomorphism.

Proof. For $j=1$ this is 9.4 and 6.16 . The general case follows by induction on $j$ and consideration of the short exact sequences

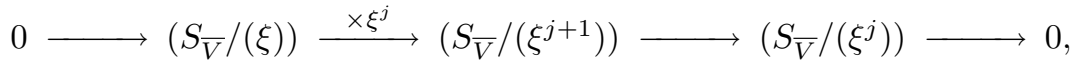


and

$0 \longrightarrow\left(S_{\overline{\mathscr{O}}_{\widehat{U} .}} /(\xi)\right) \stackrel{\times \xi^{j}}{\longrightarrow}\left(S_{\overline{\mathscr{O}}_{\widehat{U} .}} /\left(\xi^{j+1}\right)\right) \longrightarrow\left(S_{\overline{\mathscr{O}}_{\widehat{U} .}} /\left(\xi^{j}\right)\right) \longrightarrow 0$

To deduce that 12.1.1 is an isomorphism, note that by the flatness of $S_{\bar{V}} /\left(\xi^{p}\right) \rightarrow$ $\mathrm{A}_{\text {cris }}(\bar{V}) / p$ 11.9, the map

$$
\begin{gathered}
\mathrm{A}_{\text {cris }}(\bar{V}) /(p) \otimes_{S_{\bar{V}} /\left(\xi^{p}\right)} G C\left(\widehat{U}_{\bar{K}}^{o}, S_{\overline{\mathscr{O}}_{\widehat{U} .}} /\left(\xi^{p}\right) \otimes L\right) \\
\qquad \\
\downarrow \\
G C\left(\widehat{U}_{\bar{K}}^{o}, \mathrm{~A}_{\text {cris }}(\bar{V}) /(p) \otimes_{S_{\bar{V}} / \xi^{p}} S_{\overline{\mathscr{O}}_{\widehat{U} .}} /\left(\xi^{p}\right) \otimes L\right)
\end{gathered}
$$

is an isomorphism. Since

$$
\mathrm{A}_{\text {cris }}(\widehat{U} .) / p \simeq \mathrm{A}_{\text {cris }}(\bar{V}) /(p) \otimes_{S_{\bar{V}} / \xi^{p}} S_{\overline{\mathscr{O}}_{\widehat{U} .}} /\left(\xi^{p}\right),
$$

theorem 12.1 follows from the fact that 12.2.1 and 12.2.4 are quasi-isomorphisms.

THEOREM 12.3. Let $L=\lim _{\longleftarrow} L_{n}$ be a smooth sheaf of $\mathbb{Z}_{p}-$ modules on $X_{\bar{K}}^{o}$ (where

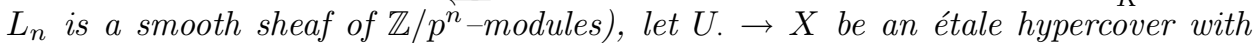
each $U_{n}$ admitting a morphism as in 2.16.1 and affine, and let $\widehat{U}$. $\rightarrow \widehat{X}$ be the p-adic completion of $U$.. Then the transformation

$$
\mathrm{A}_{\text {cris }}(\bar{V}) \otimes_{\mathbb{Z}_{p}} G C\left(U_{\cdot, \bar{K}}^{o}, L\right) \rightarrow G C\left(\widehat{U}_{\bar{K}}^{o}, L \otimes_{\mathbb{Z}_{p}} \mathrm{~A}_{\text {cris }}(\widehat{U} .)\right)
$$

is an almost quasi-isomorphism.

ProOF. The map of projective systems of complexes

$$
\left\{\mathrm{A}_{\text {cris }}(\bar{V}) \otimes_{\mathbb{Z}_{p}} G C\left(U_{\cdot, \bar{K}}^{o}, L_{n}\right)\right\}_{n} \rightarrow\left\{G C\left(\widehat{U}_{\bar{K}}^{o}, L_{n} \otimes_{\mathbb{Z}_{p}} \mathrm{~A}_{\text {cris }}(\widehat{U} .)\right)\right\}_{n}
$$

is an almost quasi-isomorphism in each degree, and therefore the assumptions of 2.23 are satisfied for both projective systems in 12.3.2 (since the left projective system clearly satisfies the assumptions of 2.23 . Therefore

$$
H^{i}\left(G C\left(\widehat{U}_{\bar{K}}^{o}, L \otimes_{\mathbb{Z}_{p}} \mathrm{~A}_{\text {cris }}(\widehat{U} .)\right)\right) \rightarrow \underset{\lim }{ } H^{i}\left(G C\left(\widehat{U}_{\bar{K}}^{o}, L_{n} \otimes_{\mathbb{Z}_{p}} \mathrm{~A}_{\text {cris }}(\widehat{U} .)\right)\right)
$$

is an almost isomorphism, and by 2.21 the composite map

$$
\begin{aligned}
\mathrm{A}_{\text {cris }}(\bar{V}) \otimes_{\mathbb{Z}_{p}} H^{i}\left(G C\left(U_{\cdot, \bar{K}}^{o}, L\right)\right) & =\underset{\lim }{\longleftarrow}\left(\mathrm{A}_{\text {cris }}(\bar{V}) \otimes H^{i}\left(G C\left(U_{\cdot, \bar{K}}^{o}, L_{n}\right)\right)\right) \\
& \rightarrow \lim ^{i} H^{i}\left(G C\left(\widehat{U}_{\bar{K}}^{o}, L_{n} \otimes_{\mathbb{Z}_{p}} \mathrm{~A}_{\text {cris }}(\widehat{U} \cdot)\right)\right)
\end{aligned}
$$

is also an almost isomorphism.

The preceding result also extends to the filtered context:

COROLlaRY 12.4. With notation as in 12.3, for any integer $n \geq 0$ the natural map

$$
\mathrm{Fil}_{\mathrm{A}_{\text {cris }}(\bar{V})}^{n} \otimes_{\mathbb{Z}_{p}} G C\left(U_{\cdot, \bar{K}}^{o}, L\right) \rightarrow G C\left(\widehat{U}_{\bar{K}}^{o}, L \otimes_{\mathbb{Z}_{p}} \operatorname{Fil}_{\mathrm{A}_{\text {cris }}\left(\widehat{U}_{.}\right)}^{n}\right)
$$

is an almost quasi-isomorphism. 
Proof. By induction on $n$. The case $n=0$ is 12.3 For the inductive step consider the short exact sequences

$$
0 \rightarrow \mathrm{Fil}_{\mathrm{A}_{\text {cris }}(\bar{V})}^{n+1} \rightarrow \mathrm{Fil}_{\mathrm{A}_{\text {cris }}(\bar{V})}^{n} \rightarrow \bar{V}^{\wedge} \cdot \xi^{[n]} \rightarrow 0,
$$

and

$$
0 \rightarrow \operatorname{Fil}_{\mathrm{A}_{\text {cris }}^{n+1}(\widehat{U} .)}^{n} \rightarrow \operatorname{Fil}_{\mathrm{A}_{\text {cris }}(\widehat{U} .)}^{n} \rightarrow \overline{\mathscr{O}}_{\widehat{U} .} \cdot \xi^{[n]} \rightarrow 0
$$

COROLlaRY 12.5. With notation as in 12.3, the natural map

$$
\widetilde{\mathrm{B}}_{\text {cris }}(\bar{V}) \otimes_{\mathbb{Q}_{p}} G C\left(U_{\cdot, \bar{K}}^{o}, L \otimes \mathbb{Q}_{p}\right) \rightarrow G C\left(\widehat{U}_{\cdot, \bar{K}}^{o}, L \otimes_{\mathbb{Z}_{p}} \widetilde{\mathrm{B}}_{\text {cris }}(\widehat{U} .)\right)
$$

is a filtered quasi-isomorphism.

12.6. We will also need variants of the above results for cohomology with partial compact support along the boundary.

For simplicity we explain just the situation for cohomology with compact support along the entire boundary, leaving to the reader to make the necessary modifications to treat partial compact support. Also fix an ordering of the boundary components $D=D_{1} \cup \cdots \cup D_{m}$. Write $\widehat{U}_{n}:=\operatorname{Spec}\left(R_{n}\right)$ and let $\bar{R}_{n}$ be the normalization of $R_{n}$ in $\widehat{U}_{n} \times_{X} X_{\bar{K}}^{o}$. For $A \subset\{1, \ldots, m\}$ let $\bar{R}_{n, A}$ be as in 8.18. We can then apply the functor $\mathrm{A}_{\text {cris }}(-)$ to get a Galois module over $\widehat{U}_{n, \bar{K}}^{o}$ which we denote by $\mathrm{A}_{\text {cris }}\left(\widehat{U}_{n} / \bar{J}_{A}\right)$. This construction is functorial in $\widehat{U}_{n}$ so we obtain a sheaf $\mathrm{A}_{\text {cris }}\left(\widehat{U}_{\cdot} / \bar{J}_{A}\right)$ on $\widehat{U}_{\bar{K}, \text { Fet }}^{o}$. Define a complex $\Phi\left(\mathrm{A}_{\text {cris }}(\widehat{U} .)\right)^{\bullet}$ by setting

$$
\Phi\left(\mathrm{A}_{\text {cris }}(\widehat{U} .)\right)^{r}:=\oplus_{A \subset\{1, \ldots, m\},|A|=r} \mathrm{~A}_{\text {cris }}\left(\widehat{U} . / \bar{J}_{A}\right)
$$

with transition maps defined as in 8.15 . Let $J_{n}^{\text {cris }} \subset \mathrm{A}_{\text {cris }}(\widehat{U}.) / p^{n+1}$ be the ideal corresponding to $D$ as in 11.19 .5

There is a similar construction with $W_{r}\left(S_{-}\right)$instead of $\mathrm{A}_{\text {cris }}(-)$. Namely, for $[n] \in \underline{\Delta}$ and $A \subset\{1, \ldots, m\}$, let $W_{r}\left(S_{\widehat{U}_{n} / \bar{J}_{A}}\right)$ denote the truncated Witt ring of the perfection of $\bar{R}_{n, A}$. Then we again obtain a complex $\left.\Phi\left(W_{r}\left(S_{\widehat{U}}\right)\right)\right)^{\bullet}$ with

$$
\Phi\left(W_{r}\left(S_{\widehat{U} .}\right)\right)^{k}:=\oplus_{A \subset\{1, \ldots, m\},|A|=k} W_{r}\left(S_{\widehat{U} \cdot / \bar{J}_{A}}\right) .
$$

Note also that there is a natural augmentation

$$
J_{r}^{\mathrm{inf}} \rightarrow \Phi\left(W_{r}\left(S_{\widehat{U} .}\right)\right)^{\bullet} .
$$

If $r=0$ we write simply $\Phi\left(S_{\widehat{U}}\right)^{\bullet}$ for $\Phi\left(W_{0}\left(S_{\widehat{U}}\right)\right)^{\bullet}$.

Lemma 12.7. For every integer $j \geq 1$ the map

$$
J_{0}^{\mathrm{inf}} / \xi^{j} J_{0}^{\mathrm{inf}} \rightarrow \Phi\left(S_{\widehat{U}}\right) /\left(\xi^{j}\right)^{\bullet} .
$$

is an almost quasi-isomorphism.

Proof. We proceed by induction on $j$.

For the case $j=1$ note that $S_{\widehat{U}} /(\xi)=\bar{R}$. $/(p)$, and if $\bar{J} \subset \bar{R}$. denotes the ideal of the boundary as in 3.15 then $J_{0}^{\text {inf }} / \xi J_{0}^{\text {inf }} \simeq \bar{J} / p \bar{J}$ (by 11.23). The result in the case $j=1$ then follows from an argument similar to the one used in the proof of 8.16 
The inductive step is obtained by devissage and noting that for every integer $j$ we have exact sequences (the first being termwise exact)

$(12.7 .2)$

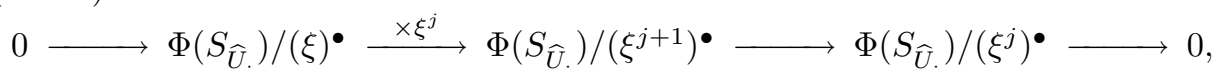

and

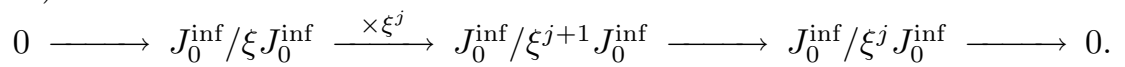

Proposition 12.8. The inclusion $J_{n}^{\text {cris }} \subset \mathrm{A}_{\text {cris }}(\widehat{U}.) / p^{n+1}=\Phi\left(\mathrm{A}_{\text {cris }}(\widehat{U} .)\right)^{0} / p^{n+1}$ induces an almost quasi-isomorphism

$$
J_{n}^{\text {cris }} \rightarrow \Phi\left(\mathrm{A}_{\text {cris }}(\widehat{U} .)\right)^{\bullet} / p^{n+1} .
$$

Proof. By devissage one is reduced to the case $n=0$. In this case 12.8.1 is obtained from the morphism of complexes

$$
J_{0}^{\inf } / \xi^{p} J_{0}^{\inf } \rightarrow \Phi\left(S_{\widehat{U}}\right) /\left(\xi^{p}\right)^{\bullet} .
$$

by making the flat base change $S_{\bar{V}} /\left(\xi^{p}\right) \rightarrow \mathrm{A}_{\text {cris }}(\bar{V}) /(p)$ (and using the identifications provided by 11.25. It therefore suffices to show that 12.8 .2 is an almost quasi-isomorphism which is 12.7

12.9. Let $L$ be a smooth sheaf of $\mathbb{Z} / p^{n+1}$-modules on $X_{\bar{K}}^{o}$, and let $\Psi(L)$ be as in 8.3 As in 8.18 .3 , there is a natural map

$$
\left.\Psi(L)\right|_{\widehat{U}_{,, \bar{K}, \mathrm{Fet}}} \rightarrow L \otimes \Phi\left(\mathrm{A}_{\mathrm{cris}}\left(\widehat{U}_{.}\right) / p^{n+1}\right) .
$$

We then obtain a diagram

$$
G C\left(\widehat{U} ., L \otimes J_{n}^{\text {cris }}\right) \rightarrow G C\left(\widehat{U} ., L \otimes \Phi\left(\mathrm{A}_{\text {cris }}(\widehat{U} .) / p^{n+1}\right)\right) \leftarrow G C\left(U_{\bar{K}}^{o}, \Psi(L)\right),
$$

where the first map is an almost quasi-isomorphism. Passing to the limit over all hypercovers $U$. we get maps

$$
\begin{gathered}
H^{*}\left(G C\left(\widehat{U} ., L \otimes J_{n}^{\text {cris }}\right)\right) \longrightarrow H^{*}\left(G C\left(\widehat{U} ., L \otimes \Phi\left(\mathrm{A}_{\text {cris }}(\widehat{U} .) / p^{n+1}\right)\right)\right) \\
H_{c}^{*}\left(X_{\bar{K}}^{o}, L\right) \otimes \mathrm{A}_{\text {cris }}(\bar{V}) / p^{n+1} .
\end{gathered}
$$

TheOREM 12.10. All the maps in 12.9.3 are almost isomorphisms.

Proof. By the usual devissage one is reduced to the case when $n=0$. As in the proof of 12.1 by the flatness of $S_{\bar{V}} /\left(\xi^{p}\right) \rightarrow \mathrm{A}_{\text {cris }}(\bar{V}) / p$ it then suffices to show that the map

$$
H_{c}^{*}\left(X_{\bar{K}}^{o}, L\right) \otimes S_{\bar{V}} / \xi^{p} \rightarrow H^{*}\left(G C\left(\widehat{U} ., L \otimes \Phi\left(S_{\widehat{U} .}\right) / \xi^{p}\right)\right)
$$

is an almost isomorphism. Filtering again by powers of $\xi$ we are reduced to 6.16 .

As in 12.3, we then also obtain results for adic sheaves: 
TheOREm 12.11. Let $L=\lim _{\longleftarrow} L_{n}$ be a smooth $\mathbb{Z}_{p}$-sheaf on $X_{\bar{K}}^{o}$. Then there is a natural almost isomorphism

$$
H^{*}\left(G C\left(\widehat{U}, L \otimes J^{\text {cris }}\right)\right) \simeq H_{c}^{*}\left(X_{\bar{K}}^{o}, L\right) \otimes \mathrm{A}_{\text {cris }}(\bar{V}) .
$$

In particular there is an actual isomorphism

$$
H^{*}\left(G C\left(\widehat{U} ., L \otimes J^{\text {cris }} \otimes_{\mathrm{A}_{\text {cris }}(\widehat{U} .)} \widetilde{\mathrm{B}}_{\text {cris }}(\widehat{U} .)\right)\right) \simeq H_{c}^{*}\left(X_{\bar{K}}^{o}, L\right) \otimes \widetilde{\mathrm{B}}_{\text {cris }}(\bar{V}) .
$$

\section{Crystalline cohomology and crystalline sheaves}

13.1. Since we are interested in $\mathbb{Q}_{p}$-coefficients and isocrystals we will work throughout with the convergent topos as opposed to the crystalline topos. The reference for the convergent topos in the non-logarithmic setting is $\mathbf{3 9}$ and in the logarithmic setting the paper 46 .

Let $X / V$ be a smooth proper scheme, $D \subset X$ a divisor with relative normal crossings defining a log structure $M_{X}$ on $X$, and let $\left(Y, M_{Y}\right)$ denote the closed fiber of $\left(X, M_{X}\right)$. Recall that the convergent topos $\left(\left(Y, M_{Y}\right) / V\right)_{\text {conv }}$ is the topos associated to the site $\operatorname{Conv}\left(\left(Y, M_{Y}\right) / V\right)$ whose objects are strict closed immersions $\left(U, M_{U}\right) \hookrightarrow\left(T, M_{T}\right)$, where $U \rightarrow X$ is an étale morphism, $M_{U}$ is the pullback of $M_{X}$, and $\left(T, M_{T}\right)$ is a formal $\log$ scheme flat over $\operatorname{Spf}(V)$ with the $\pi$-adic topology. The diagram

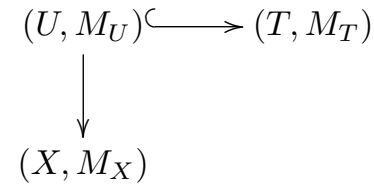

is called an enlargement. We often write simply $\left(T, M_{T}\right)$ for such an enlargement. A morphism $\left(\left(U^{\prime}, M_{U^{\prime}}\right) \hookrightarrow\left(T^{\prime}, M_{T^{\prime}}\right)\right) \rightarrow\left(\left(U, M_{U}\right) \hookrightarrow\left(T, M_{T}\right)\right)$ is a commutative diagram of $\log$ schemes

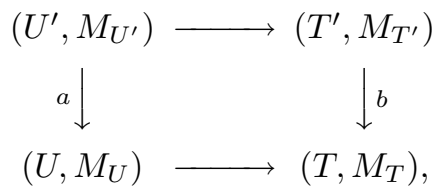

where $a$ is a $X$-morphism. Coverings are defined to be collections of morphisms $\left\{\left(T_{i}, M_{T_{i}}\right) \rightarrow\left(T, M_{T}\right)\right\}_{i}$ such that the map $\amalg T_{i} \rightarrow T$ is an étale covering.

We write $\mathscr{O}_{\left(Y, M_{Y}\right) / V}$ for the structure sheaf $\left(T, M_{T}\right) \mapsto \Gamma\left(T, \mathscr{O}_{T}\right)$ and $\mathscr{K}_{\left(Y, M_{Y}\right) / V}$ for the sheaf $\left(T, M_{T}\right) \mapsto \Gamma\left(T, \mathscr{O}_{T}\right) \otimes \mathbb{Q}$. For a sheaf $\mathscr{F}$ of $\mathscr{K}_{\left(Y, M_{Y}\right) / V}$-modules and an object $\left(T, M_{T}\right)$ of $\operatorname{Conv}\left(\left(Y, M_{Y}\right) / V\right)$ we write $\mathscr{F}_{T}$ for the induced sheaf on $T_{\text {et }}$. An isocrystal is a sheaf $\mathscr{F}$ of $\mathscr{K}_{\left(Y, M_{Y}\right) / V}$-modules such that for every $\left(T, M_{T}\right)$ the sheaf $\mathscr{F}_{T}$ is coherent and such that for any morphism $f:\left(T^{\prime}, M_{T^{\prime}}\right) \rightarrow\left(T, M_{T}\right)$ in $\operatorname{Conv}\left(\left(Y, M_{Y}\right) / V\right)$ the induced morphism $f^{*} \mathscr{F}_{T} \rightarrow \mathscr{F}_{T^{\prime}}$ is an isomorphism.

Let $W$ denote the ring of Witt vectors of the residue field $k$ of $W$. Then we can also consider the convergent topos $\left(\left(Y, M_{Y}\right) / W\right)_{\text {conv }}$. There is a natural morphism of topoi

$$
\pi:\left(\left(Y, M_{Y}\right) / V\right)_{\mathrm{conv}} \rightarrow\left(\left(Y, M_{Y}\right) / W\right)_{\mathrm{conv}} .
$$

For any isocrystal $\mathscr{F}$ in $\left(\left(Y, M_{Y}\right) / W\right)_{\text {conv }}$ the induced morphism

$$
H^{*}\left(\left(\left(Y, M_{Y}\right) / W\right)_{\mathrm{conv}}, \mathscr{F}\right) \rightarrow H^{*}\left(\left(\left(Y, M_{Y}\right) / V\right)_{\mathrm{conv}}, \pi^{*} \mathscr{F}\right)
$$


is an isomorphism (without log structures this is [40,3.2], and the same argument yields the case with log structures).

Note that Frobenius induces a morphism of topoi

$$
\sigma:\left(\left(Y, M_{Y}\right) / W\right)_{\mathrm{conv}} \rightarrow\left(\left(Y, M_{Y}\right) / W\right)_{\mathrm{conv}} .
$$

We can therefore define an $F$-isocrystal to be a pair $\left(\mathscr{F}, \varphi_{\mathscr{F}}\right)$ where $\mathscr{F}$ is an isocrystal in $\left(\left(Y, M_{Y}\right) / W\right)$ and $\varphi_{\mathscr{F}}: \sigma^{*} \mathscr{F} \rightarrow \mathscr{F}$ is an isomorphism.

13.2. Let $\left(\widehat{X}, M_{\widehat{X}}\right)$ denote the $p$-adic completion of $\left(X, M_{X}\right)$ so that $\left(Y, M_{Y}\right) \hookrightarrow$ $\left(\widehat{X}, M_{\widehat{X}}\right)$ is an object of $\operatorname{Conv}\left(\left(Y, M_{Y}\right) / V\right)$. As usual, there is then a morphism of sites

$$
\operatorname{Et}(\widehat{X}) \rightarrow \operatorname{Conv}\left(\left(Y, M_{Y}\right) / V\right), \quad(T \rightarrow \widehat{X}) \mapsto\left(\left(T \times_{X} Y,\left.M_{Y}\right|_{T \times_{X} Y}\right) \hookrightarrow\left(T,\left.M_{\widehat{X}}\right|_{T}\right)\right)
$$

inducing a morphism of topoi

$$
u_{X / V}:\left(\left(Y, M_{Y}\right) / V\right)_{\text {conv }} \rightarrow \widehat{X}_{\text {et }} .
$$

As in the classical case, the category of isocrystals is identified with the category of coherent $\mathscr{K}_{\widehat{X}}$-modules $\mathcal{F}$ with an integrable connection

$$
\nabla: \mathcal{F} \rightarrow \mathcal{F} \otimes_{\mathscr{O}_{\widehat{X}}} \Omega_{\left(\widehat{X}, M_{\widehat{X}}\right) / V}^{1}
$$

satisfying a $p$-adic convergence condition. Write $D R(\mathcal{F}, \nabla)$ for the associated de Rham complex. By [46, 2.3.5] there is then a canonical quasi-isomorphism $R u_{X / V *} \mathscr{F} \simeq D R(\mathcal{F}, \nabla)$. Note also that since $X / V$ is proper an isocrystal is induced by a unique coherent $\mathscr{O}_{X_{K}}$-module with integrable connection satisfying a convergence condition, and the de Rham complex $D R(\mathcal{F}, \nabla)$ computes the de Rham cohomology of this module with integrable connection on the generic fiber by [11. III.5.1.2].

13.3. Let $D=E \cup F$ be a decomposition of the boundary of $X$. We can then define an isocrystal $J_{E}$ as follows. First let $\bar{M}$ denote the sheaf on $\operatorname{Conv}\left(\left(Y, M_{Y}\right) / W\right)$ which to any $\left(U, M_{U}\right) \hookrightarrow\left(T, M_{T}\right)$ associates $\Gamma\left(T, \bar{M}_{T}\right)=\Gamma\left(U, \bar{M}_{U}\right)$. For any geometric point $\bar{t} \rightarrow U$ the stalk $\bar{M}_{U}$ is canonically isomorphic to the free monoid with generators indexed by the irreducible components of the inverse image of $D$ in $\operatorname{Spec}\left(\mathscr{O}_{U, \bar{t}}\right)$. In particular, there is a canonical submonoid $K_{E, \bar{t}} \subset \bar{M}_{\bar{t}}$ generated by the sum of the generators of $\bar{M}_{\bar{t}}$ corresponding to those irreducible components which are in $E$. One verifies immediately that there is a subsheaf of monoids $K_{E} \subset \bar{M}$ whose stalks at each geometric point $\bar{t}$ agrees with $K_{E, \bar{t}}$. Define $J_{E}^{\prime} \subset \mathscr{O}_{\left(Y, M_{Y}\right) / V}$ to be the sheaf of ideals whose restriction to any $\left(T, M_{T}\right)$ is generated by the images of sections $m \in M_{T}$ mapping to $K_{E}$ in $\bar{M}_{T}$.

If $U \rightarrow Y$ is étale and admits a chart 2.16.1 and if $\left(U, M_{U}\right) \hookrightarrow\left(Z, M_{Z}\right)$ is an embedding into a formally log smooth $W$-scheme, then a chart for $\left(U, M_{U}\right)$ extends to a chart

$$
\left(Z, M_{Z}\right) \rightarrow \operatorname{Spf}\left(W\left[X_{1}, \ldots, X_{d}\right]\right) .
$$

If $E$ is defined by the inverse image of $\left\{X_{1} \cdots X_{s}=0\right\}$ then $J_{E, Z} \subset \mathscr{O}_{Z}$ is simply the ideal defined by the image of $X_{1} \cdots X_{s}$. This implies that if $f:\left(Z^{\prime}, M_{Z^{\prime}}\right) \rightarrow$ $\left(Z, M_{Z}\right)$ is a morphism of $\log$ smooth objects of $\operatorname{Conv}\left(\left(Y, M_{Y}\right) / W\right)$ then the natural map $f^{*} J_{E, Z} \rightarrow J_{E, Z^{\prime}}$ is an isomorphism. In particular, applying this to the completion of $\left(Z, M_{Z}\right) \times_{V}\left(Z, M_{Z}\right)$ (this should be the "strictification" of the diagonal; 
see [29, 5.8] and [41, Appendix A]) we see that $J_{E, Z}$ has a canonical integrable connection defining an isocrystal $J_{E}$. Concretely, in local coordinates 13.3.1 this just amounts to saying that the connection

$$
d: W\left[X_{1}, \ldots, X_{d}\right] \rightarrow W\left[X_{1}, \ldots, X_{d}\right] \otimes_{W}\left(\oplus_{i} W \cdot d \log X_{i}\right)
$$

sends the ideal $\left(X_{1} \cdots X_{s}\right)$ to $\left(X_{1} \cdots X_{s}\right) \otimes_{W}\left(\oplus_{i} W \cdot d \log X_{i}\right)$ which is immediate. Let $J_{E}$ denote the corresponding isocrystal on $\operatorname{Conv}\left(\left(Y, M_{Y}\right) / W\right)$. There is a natural surjection $J_{E} \rightarrow J_{E}^{\prime}$ which is an isomorphism on formally smooth objects of $\operatorname{Conv}\left(\left(Y, M_{Y}\right) / W\right)$ (the kernel is "parasitic"). If $E=D$ we also sometimes write just $J_{X}$ for $J_{E}$.

Lemma 13.4. Let $\left(U, M_{U}\right) \hookrightarrow\left(T, M_{T}\right)$ be an object of $\operatorname{Conv}\left(\left(Y, M_{Y}\right) / W\right)$ with $T$ affine, and assume given a chart $c: \mathbb{N}^{r} \rightarrow M_{U}$ such that $E$ is defined by the image $f$ of $(1, \ldots, 1,0, \ldots, 0)$ ( 1 's in the first $s$ places). Assume that for some lifting $\tilde{c}: \mathbb{N}^{r} \rightarrow M_{T}$ of the chart the image $\tilde{f}$ of $(1, \ldots, 1,0, \ldots, 0)$ in $\mathscr{O}_{T}$ is a non-zero divisor. Then the map $J_{E}(T) \rightarrow J_{E}^{\prime}(T)$ is an isomorphism.

PROOF. This follows immediately from the preceding discussion.

Corollary 13.5. Let $U=\operatorname{Spec}(R) \rightarrow X$ be an étale morphism admitting a morphism as in 2.16.1. Then the natural map $J_{E}\left(\mathrm{~A}_{\text {cris }}\left(R^{\wedge}\right)\right) \rightarrow J_{E}^{\prime}\left(\mathrm{A}_{\text {cris }}\left(R^{\wedge}\right)\right)$ is an isomorphism.

13.6. Note that there is a natural map $\sigma^{*} J_{E} \rightarrow J_{E}$, which is not usually an isomorphism. Thus for any $F$-isocrystal $\left(\mathscr{F}, \varphi_{\mathscr{F}}\right)$ we can consider the cohomology with compact support along $E$

$$
H^{*}\left(\left(\left(Y, M_{Y}\right) / W\right)_{\text {conv }}, \mathscr{F} \otimes J_{E}\right)
$$

which has a semi-linear Frobenius endomorphism. There is a canonical pairing

$$
\begin{gathered}
H^{*}\left(\left(\left(Y, M_{Y}\right) / W\right)_{\text {conv }}, \mathscr{F} \otimes J_{E}\right) \otimes H^{2 d-*}\left(\left(\left(Y, M_{Y}\right) / W\right)_{\text {conv }}, \mathscr{F}^{*} \otimes J_{F}\right) \\
\downarrow \\
H^{2 d}\left(\left(\left(Y, M_{Y}\right) / W\right)_{\text {conv }}, J_{X}\right)
\end{gathered}
$$

compatible with Frobenius.

LEMma 13.7. This pairing is a perfect pairing.

Proof. It suffices to verify this after base changing to $K$. Thus we may consider the topos $\left(\left(Y, M_{Y}\right) / V\right)_{\text {conv }}$ instead of $\left(\left(Y, M_{Y}\right) / W\right)_{\text {conv }}$. In this case it suffices to show the analogous result for de Rham cohomology of the generic fiber. Write also $J_{E}$ for the ideal in $\mathscr{O}_{X_{K}}$ defining $E_{K}$. Then we wish to show that the natural map

$$
R \Gamma\left(D R\left(\mathcal{F} \otimes J_{E}, \nabla\right)\right) \rightarrow \mathrm{RHom}\left(R \Gamma\left(D R\left(\mathcal{F}^{*} \otimes J_{F}, \nabla\right)\right), \Omega_{X_{K}}^{d}\right)
$$

is an isomorphism or better the local version that

$$
D R\left(\mathcal{F} \otimes J_{E}, \nabla\right) \rightarrow \mathscr{R} H o m\left(D R\left(\mathcal{F}^{*} \otimes J_{F}, \nabla\right), \Omega_{X_{K}}^{d}\right)
$$

is an isomorphism which is immediate.

LEMma 13.8 (46, 2.3.6]). Let $\mathscr{F}$ be an isocrystal. Associated to the lifting $\left(\widehat{X}, M_{\widehat{X}}\right)$ is a canonical resolution $\mathscr{F} \rightarrow \mathbb{R}^{\bullet}$ such that each $\mathbb{R}^{i}$ is acyclic for the projection $u_{X / V *}$. This resolution is functorial in $\mathscr{F}$. 
Proof. Let $T$ denote the object of the convergent site of $\left(Y, M_{Y}\right)$ given by $\left(Y, M_{Y}\right) \hookrightarrow\left(\widehat{X}, M_{\widehat{X}}\right)$. There is then a diagram of topoi

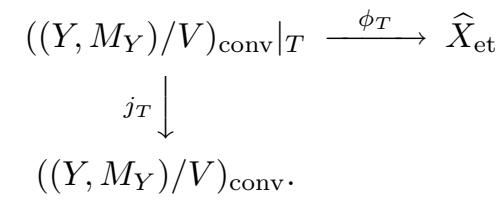

As explained in [46, 2.3.6] (in loc. cit. our $\mathbb{R}^{j}$ is denoted $\omega_{P}^{j}(\mathcal{E})$ ) the resolution is then given by $\mathbb{R}^{j}:=j_{T *}\left(\phi_{T}^{*} \Omega_{\left(X, M_{X}\right) / V}^{j} \otimes j_{T}^{*} \mathscr{F}\right)$.

REMARK 13.9. Note that since $\mathscr{F}$ is an isocrystal, there is a canonical isomorphism $j_{T}^{*} \mathscr{F} \simeq \phi_{T}^{*} \mathcal{F}$. Therefore if $\mathcal{F}$ comes is equipped with a filtration $A^{\bullet}$ satisfying Griffiths transversality (see 13.11 .1 below), then each of the sheaves $\mathbb{R}^{i}$ is naturally filtered by the subsheaves $j_{T *}\left(\phi_{T}^{*} \Omega_{\left(X, M_{X}\right) / V}^{j} \otimes \phi_{T}^{*} A^{i}\right)$ and $\mathbb{R}^{\bullet}$ is a filtered complex (where $j_{T *}\left(\phi_{T}^{*} \Omega_{\left(X, M_{X}\right) / V}^{j} \otimes \phi_{T}^{*} A^{i}\right)$ lies in the $(i+j)$-th step of the filtration). Furthermore, if $(\mathcal{F}, \nabla)$ is the module with integrable connection corresponding to $\mathscr{F}$ then the natural map $\left.(\mathcal{F}, \nabla) \rightarrow \mathbb{R}^{\bullet}\right|_{\widehat{X}}$ is a filtered quasi-isomorphism.

13.10. Let $\mathscr{F} \rightarrow \mathbb{R}^{\bullet}$ be the resolution corresponding to our lifting $\left(X, M_{X}\right) / V$. The sheaves $\mathbb{R}^{i}$ are not isocrystals, but still the value $\mathbb{R}^{i}\left(Z, M_{Z}\right)$ of $\mathbb{R}^{i}$ on any affine enlargment $\left(U, M_{U}\right) \hookrightarrow\left(Z, M_{Z}\right)$, with $\left(Z, M_{Z}\right)$ formally smooth over $V$, has a canonical integrable connection

$$
\mathbb{R}^{i}\left(Z, M_{Z}\right) \rightarrow \mathbb{R}^{i}\left(Z, M_{Z}\right) \otimes \Omega_{\left(Z, M_{Z}\right) / V}^{1}
$$

This follows from the construction of $\mathbb{R}^{i}$ (see the proof of [46, 2.3.5] and without log structures [39, 0.5.4]). Moreover, by the proof of the convergent Poincaré lemma [46, 2.3.5 (2)], the complex $\mathbb{R}^{\bullet}\left(Z, M_{Z}\right)$ is a resolution of $\mathscr{F}\left(Z, M_{Z}\right)$.

13.11. As in [14 there is a category $M F_{X}^{\nabla}(\Phi)$ defined as follows. Fix a geometric generic point $\eta: \operatorname{Spec}(\Omega) \rightarrow X$, and let $\widehat{\Omega}$ denote the $p$-adic completion. If $\mathscr{F}$ is an isocrystal on $\left(Y, M_{Y}\right) / W$, let $\left(\mathcal{F}, \nabla_{\mathcal{F}}\right)$ denote the module with logarithmic connection on $\left(X_{K}, M_{X_{K}}\right)$ obtained by evaluating $\mathscr{F}$ on the enlargement $\left(Y, M_{Y}\right) \hookrightarrow\left(X^{\wedge}, M_{X^{\wedge}}\right)$, where $\left(X^{\wedge}, M_{X^{\wedge}}\right)$ denotes the $p$-adic completion of $\left(X, M_{X}\right) / V$. The category $M F_{X}^{\nabla}(\Phi)$ is defined to be the category of triples $\left(\mathscr{F}, \varphi_{\mathscr{F}}, \operatorname{Fil}_{\mathcal{F}}\right)$, where $\left(\mathscr{F}, \varphi_{\mathscr{F}}\right)$ is an $F$-isocrystal on $\left(Y, M_{Y}\right) / W$ and $\operatorname{Fil}_{\mathcal{F}}$ is a decreasing filtration on $\mathcal{F}$ satisfying Griffiths transversality

$$
\nabla_{\mathcal{F}}\left(\mathrm{Fil}_{\mathcal{F}}^{i}\right) \subset \operatorname{Fil}_{\mathcal{F}}^{i-1} \otimes \Omega_{\left(X_{K}, M_{X_{K}}\right) / K}^{1}
$$

13.12. If $\left(\mathscr{F}, \varphi_{\mathscr{F}}, \operatorname{Fil}_{\mathcal{F}}\right) \in M F_{X}^{\nabla}(\Phi)$ and $\operatorname{Spec}(R) \rightarrow X$ a disjoint union of open subsets of $X$ admitting morphisms as in 2.16.1 we can evaluate $\mathscr{F}$ on the enlargement 11.17 .2 to get a $\mathrm{A}_{\text {cris }}\left(R^{\wedge}\right) \otimes \mathbb{Q}-$ module $\mathscr{F}\left(\left(\operatorname{Spec}\left(\mathrm{A}_{\text {cris }}\left(R^{\wedge}\right)\right), M_{\mathrm{A}_{\text {cris }}\left(R^{\wedge}\right)}\right)\right)$. Inverting $t \in \mathrm{A}_{\text {cris }}\left(R^{\wedge}\right)$, we get a $\mathrm{B}_{\text {cris }}\left(R^{\wedge}\right)$-module which we denote simply by $\mathscr{F}\left(\mathrm{B}_{\text {cris }}\left(R^{\wedge}\right)\right)$. The $F$-isocrystal structure $\varphi_{\mathscr{F}}$ induces a semi-linear automorphism of the $\mathrm{B}_{\text {cris }}\left(R^{\wedge}\right)$-module $\mathscr{F}\left(\mathrm{B}_{\text {cris }}\left(R^{\wedge}\right)\right)$.

The $\mathrm{B}_{\text {cris }}\left(R^{\wedge}\right)$-module $F\left(\mathrm{~B}_{\text {cris }}\left(R^{\wedge}\right)\right)$ also has a natural filtration $\operatorname{Fil}_{F\left(\mathrm{~B}_{\text {cris }}\left(R^{\wedge}\right)\right)}$ defined as follows. Since $\left(X, M_{X}\right) / V$ is smooth, we can choose a morphism

$$
r:\left(\operatorname{Spec}\left(\mathrm{A}_{\text {cris }}\left(R^{\wedge}\right)\right), M_{\mathrm{A}_{\text {cris }}\left(R^{\wedge}\right)}\right) \rightarrow\left(\operatorname{Spec}(R), M_{\operatorname{Spec}(R)}\right)
$$


such that the diagram

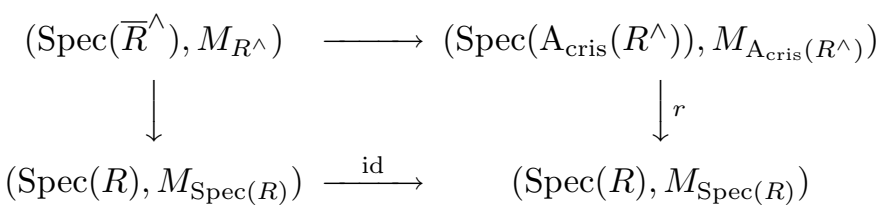

commutes. The choice of such an $r$ gives an isomorphism

$$
\sigma_{r}: F\left(\mathrm{~B}_{\text {cris }}\left(R^{\wedge}\right)\right) \simeq \mathcal{F}(\operatorname{Spec}(R)) \otimes_{R} \mathrm{~B}_{\text {cris }}\left(R^{\wedge}\right),
$$

and we define $\operatorname{Fil}_{F\left(\mathrm{~B}_{\text {cris }}\left(R^{\wedge}\right)\right)}$ to be the tensor product filtration of $\operatorname{Fil}_{\mathcal{F}(\operatorname{Spec}(R))}$ and the filtration $\mathrm{Fil}_{\mathrm{B}_{\text {cris }}\left(R^{\wedge}\right)}$ on $\mathrm{B}_{\text {cris }}\left(R^{\wedge}\right)$.

LEMmA 13.13. The filtration $\mathrm{Fil}_{F\left(\mathrm{~B}_{\mathrm{cris}}\left(R^{\wedge}\right)\right)}$ is independent of the choice of $r$.

Proof. See [50] or [41, 5.8].

13.14. The module $F\left(\mathrm{~B}_{\text {cris }}\left(R^{\wedge}\right)\right)$ also comes equipped with a continuous action of $\operatorname{Gal}\left(\bar{R}^{\wedge} / R^{\wedge}\right)$ which commutes with the Frobenius automorphism induced by the $F$-isocrystal structure as well as the filtration. As in 11.16 this $\operatorname{Gal}\left(\bar{R}^{\wedge} / R^{\wedge}\right)^{-}$ module $F\left(\mathrm{~B}_{\text {cris }}\left(R^{\wedge}\right)\right)$ is functorial for morphisms $s: \operatorname{Spec}\left(\Omega^{\prime}\right) \rightarrow \operatorname{Spec}(\Omega)$ of geometric generic points of $\operatorname{Spec}(R)$.

If $U=\operatorname{Spec}(R) \rightarrow X$ is a disjoint union of open subsets of $X$ admitting a morphism as in 2.16.1 and $\left(F, \varphi_{F}, \operatorname{Fil}_{\mathcal{F}}\right) \in M F_{X}^{\nabla}(\Phi)$, we write $F\left(\mathrm{~B}_{\text {cris }}\left(U^{\wedge}\right)\right.$ ) (or $F\left(\mathrm{~B}_{\text {cris }}\left(R^{\wedge}\right)\right)$ ) for the filtered Galois module with semi-linear automorphisms on $U_{K}^{\wedge o}:=\operatorname{Spec}\left(R^{\wedge}\right) \times_{X} X_{K}^{o}$ obtained from the construction 13.12 on each connected component.

13.15. If $L$ is a smooth $\mathbb{Q}_{p}$-sheaf on $X_{K}^{o}$ and $U \rightarrow X$ is étale and admitting a morphism 2.16.1 the pullback of $L$ to $U_{K}^{\wedge o}$ is a Galois module on $U_{K}^{\wedge o}$ which we denote by $L_{U_{K}^{\wedge}{ }^{o}}$. Define an association $\iota$ between $\left(F, \varphi_{F}, \operatorname{Fil}_{\mathcal{F}}\right) \in M F_{X}^{\nabla}(\Phi)$ and a smooth $\mathbb{Q}_{p}$-sheaf $L$ on $X_{K}^{o}$ to be a collection of isomorphisms of Galois modules, one for each étale $U \rightarrow X$ as in 2.16.1.

$$
\iota_{U}: F\left(\mathrm{~B}_{\text {cris }}\left(U^{\wedge}\right)\right) \simeq L_{U_{K}^{\wedge}} \otimes \mathrm{B}_{\text {cris }}\left(U^{\wedge}\right)
$$

compatible with the semi-linear Frobenius automorphisms, and the filtrations. Furthermore, we require that the isomorphisms $\iota_{U}$ be compatible with morphisms over $X$. A smooth sheaf $L$ on $X_{K}$ is called crystalline if it is associated to some object in $M F_{X}^{\nabla}(\Phi)$.

Remark 13.16. Crystalline sheaves arise as follows. Let $X$ and $Y$ be smooth proper $V$-schemes with normal crossing divisors $D \subset X$ and $E \subset Y$, and let $f: Y \rightarrow X$ be a morphism with $f^{-1}(D) \subset E$. Assume that the induced morphism of $\log$ schemes $f:\left(Y, M_{Y}\right) \rightarrow\left(X, M_{X}\right)$ (log structures defined by $E$ and $D$ ) is of Cartier type [29, 4.8]. For example, if étale locally on $X$ and $Y$ we can describe the morphism as

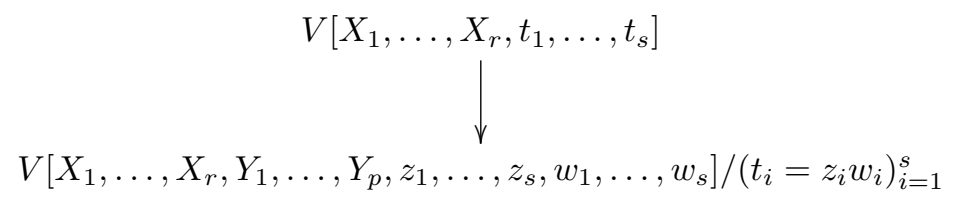


such that $D$ is the divisor defined by $t_{1} \cdots t_{s}=0$ and $E$ is the divisor defined by $z_{1} \ldots, z_{s} w_{1} \cdots w_{s}=0$. Then for any crystalline sheaf $L$ on $Y$ associated to some object $\left(F, \varphi_{F}, \operatorname{Fil}_{\mathcal{F}}\right) \in M F_{Y}^{\nabla}(\Phi)$ and integer $i$ the direct image $R^{i} f_{*} L$ on $X_{\bar{K}}^{o}$ is crystalline and is associated to $R^{i} f_{*} F$ (with the filtration and $F$-isocrystal structure induced by that on $F$ ). This is shown in [14,6.3]. In particular we can take $L=\mathbb{Q}_{p}$ which is associated to the trivial $F$-isocrystal.

13.17. Let $L$ be a crystalline sheaf associated to $\left(F, \varphi_{F}, \operatorname{Fil}_{\mathcal{F}}\right)$. For any decomposition $D=E \cup F$ we now define a transformation

$$
\alpha: \widetilde{\mathrm{B}}_{\mathrm{cris}}(\bar{V}) \otimes_{K} H^{*}\left(\left(\left(Y, M_{Y}\right) / V\right)_{\mathrm{conv}}, F \otimes J_{E}\right) \rightarrow \widetilde{\mathrm{B}}_{\mathrm{cris}}(\bar{V}) \otimes_{\mathbb{Q}_{p}} H_{E, F}^{*}\left(X_{\bar{K}, \mathrm{et}}^{o}, L\right)
$$

compatible with Galois action, filtrations, and Frobenius as well as cup-products.

For this choose an étale hypercover $U . \rightarrow X$ with each $U_{n}$ admitting a morphism 2.16.1. Let $Z$. $\rightarrow Y$ be the reduction of $U$.

For any étale morphism $Z \rightarrow Y$, let $h_{Z}$ denote the sheaf on $\operatorname{Conv}\left(\left(Y, M_{Y}\right) / V\right)$ which to any $\left(\left(U, M_{U}\right) \hookrightarrow\left(T, M_{T}\right)\right.$ associates the set of morphisms $U \rightarrow Z$ over $Y$. Then the localized topos $\left.\left(\left(Y, M_{Y}\right) / V\right)_{\text {conv }}\right|_{h_{Z}}$ is canonically equivalent to the topos $\left(\left(Z, M_{Z}\right) / V\right)_{\text {conv }}$. Write $\mathrm{A}_{\text {cris }}\left(U^{\wedge}\right)$ for the simplicial object of $\operatorname{Conv}\left(\left(Y, M_{Y}\right) / V\right)$ defined by the enlargements $\mathrm{A}_{\text {cris }}\left(U_{n}^{\wedge}\right)$. Then there is a natural morphism of sheaves $\mathrm{A}_{\text {cris }}\left(U^{\wedge}\right) \rightarrow h_{Z}$. which induces a morphism of topoi

$$
\pi:\left.\left.\left(\left(Y, M_{Y}\right) / V\right)_{\text {conv }}\right|_{\mathrm{A}_{\text {cris }}\left(U^{\wedge}\right)} \rightarrow\left(\left(Y, M_{Y}\right) / V\right)_{\text {conv }}\right|_{h_{Z}} .
$$

This morphism sits naturally in a commutative diagram of topoi

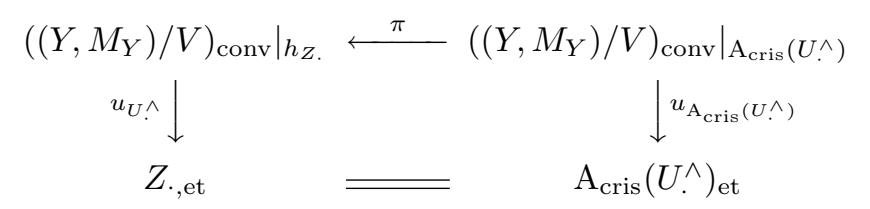

Let $\pi_{0}\left(U_{\cdot, \bar{K}}\right)$ denote the simplicial set

$$
[n] \mapsto\left\{\text { the set of connected components of } U_{n, \bar{K}}\right\} \text {. }
$$

We view $\pi_{0}\left(U_{\cdot, \bar{K}}\right)$ as a simplicial topos with the discrete topology. Let $\operatorname{Vec}_{K}^{\pi_{0}\left(U_{\cdot, \bar{K}}\right)}$ denote the category of sheaves of $K$-vector spaces in this topos. Concretely, $\operatorname{Vec}_{K}^{\pi_{0}(U, \bar{K})}$ assigns in a functorial manner a $K$-vector space to each connected component of each $U_{n, \bar{K}}$. Taking global section defines a functor

$$
\Gamma_{\bullet}:\left(\mathscr{K}_{X / V^{-}} \text {modules in }\left.\left(\left(Y, M_{Y}\right) / V\right)_{\text {conv }}\right|_{h_{Z} .}\right) \rightarrow \operatorname{Vec}_{K}^{\pi_{0}(U \cdot, \bar{K})} .
$$

We can also consider the evaluation on the enlargements $\mathrm{A}_{\text {cris }}\left(U_{n}^{\wedge}\right)$ which gives a functor

$$
\operatorname{ev}_{\mathrm{A}_{\text {cris }}(U \wedge)}:\left(\mathscr{K}_{X / V^{-}} \text {modules in }\left.\left(\left(Y, M_{Y}\right) / V\right)_{\text {conv }}\right|_{h_{Z} .}\right) \rightarrow \operatorname{Vec}_{K}^{\pi_{0}(U, \bar{K})} .
$$

There is a natural transformation of functors $\Gamma_{\bullet} \rightarrow \operatorname{ev}_{\mathrm{A}_{\text {cris }}\left(U^{\wedge}\right)}$. Since the functor $\mathrm{ev}_{\mathrm{A}_{\text {cris }}\left(U^{\wedge}\right)}$ is exact on quasi-coherent isocrystals this induces a natural morphism

$$
R \Gamma \bullet\left(F \otimes J_{E}\right) \rightarrow \operatorname{ev}_{\mathrm{A}_{\text {cris }}\left(U^{\wedge}\right)}\left(F \otimes J_{E}\right)
$$

in the derived category $D\left(\operatorname{Vec}_{K}^{\pi_{0}\left(U_{,, \bar{K}}\right)}\right)$. 
We can also consider the topos $\left(\left(Y, M_{Y}\right) / W\right)_{\text {conv }} \mid h_{Z}$. which sits in a diagram $(13.17 .8)$

$\left.\left.\left(\left(Y, M_{Y}\right) / W\right)_{\mathrm{conv}}\right|_{h_{Z} .} \leftarrow\left(\left(Y, M_{Y}\right) / V\right)_{\mathrm{conv}}\left|h_{Z .} \leftarrow\left(\left(Y, M_{Y}\right) / V\right)_{\mathrm{conv}}\right|_{\mathrm{A}_{\mathrm{cris}}\left(U^{\wedge}\right)}\right)$ If

$$
\Gamma_{\bullet}^{\prime}:\left(\mathscr{K}_{\left(Y, M_{Y}\right) / W^{-}} \text {modules in }\left(\left(Y, M_{Y}\right) / W\right)_{\text {conv }} \mid h_{Z}\right) \rightarrow \operatorname{Vec}_{K}^{\pi_{0}\left(U_{\cdot, \bar{K}}\right)}
$$

denotes the global section functor we then obtain a diagram

$$
R \Gamma_{\bullet}^{\prime}\left(F \otimes J_{E}\right) \rightarrow R \Gamma_{\bullet}\left(F \otimes J_{E}\right) \rightarrow \mathrm{ev}_{\mathrm{A}_{\text {cris }}\left(U^{\wedge}\right)}\left(F \otimes J_{E}\right) .
$$

It follows from the construction that the composite

$$
R \Gamma_{\bullet}^{\prime}\left(F \otimes J_{E}\right) \rightarrow \operatorname{ev}_{\mathrm{A}_{\text {cris }}\left(U^{\wedge}\right)}\left(F \otimes J_{E}\right)
$$

is compatible with the Frobenius actions. This map induces a morphism in the category $D\left(\operatorname{Vec}_{K}^{\pi_{0}\left(U_{, \bar{K}}\right)}\right)$

$$
R \Gamma_{\bullet}^{\prime}\left(F \otimes J_{E}\right) \rightarrow\left(F \otimes J_{E}\right)\left(\mathrm{B}_{\text {cris }}\left(U_{.}^{\wedge}\right)\right) .
$$

13.18. Next we need some facts about Galois representations. Let $\operatorname{Rep}_{K}^{\text {cts }}(\Delta$.) denote the simplicial category whose fiber over $[n] \in \underline{\Delta}$ is the category of collections $\left(L_{i}\right)_{i \in \pi_{0}\left(U_{n, \bar{K}}\right)}$, where each $L_{i}$ is a continuous representation of the fundamental group (with respect to the fixed generic base point) of the $i$-th connected component of $U_{\bar{K}}^{o}$. The category $\operatorname{Rep}_{K}^{\text {cts }}(\Delta$.) is easily seen to be an abelian tensor category with enough injectives. We denote by $D\left(\operatorname{Rep}_{K}^{\text {cts }}(\Delta).\right)$ its derived category.

The functor $\operatorname{ev}_{\mathrm{A}_{\text {cris }}\left(U^{\wedge}\right)}$ is naturally viewed as taking values in $\operatorname{Rep}_{K}^{\text {cts }}(\Delta$.), and the transformation of functors $\Gamma_{\bullet} \rightarrow \mathrm{ev}_{\mathrm{A}_{\text {cris }}\left(U^{\wedge}\right)}$ in fact factors through the subfunctor of invariants in $\mathrm{ev}_{\mathrm{A}_{\text {cris }}\left(U^{\wedge}\right)}$.

From this it follows that the morphism 13.17 .12 induces a morphism in the derived category $D\left(\operatorname{Vec}_{K}^{\pi_{0}\left(U_{\cdot, \bar{K}}\right)}\right)$

$$
R \Gamma_{\bullet}\left(F \otimes J_{E}\right) \rightarrow G C\left(\left(F \otimes J_{E}\right)\left(\mathrm{B}_{\mathrm{cris}}\left(U_{.}^{\wedge}\right)\right)\right)
$$

compatible with Frobenius.

Lemma 13.19. The induced morphism

$$
R \Gamma \bullet\left(F \otimes J_{E}\right) \rightarrow G C\left(\left(F \otimes J_{E}\right)\left(\mathrm{B}_{\text {cris }}\left(U_{.}^{\wedge}\right)\right)\right)
$$

extends canonically to a morphism in the filtered derived category.

Proof. The map can be represented on the level of filtered complexes as follows. Let $F \otimes J_{E} \rightarrow \mathbb{R}^{\bullet}$ be the resolution given by 13.8 . By 13.9 the complex $R \Gamma \bullet\left(F \otimes J_{E}\right)$ can then be represented, as a filtered complex, by $\mathbb{R}^{\bullet}\left(U^{\wedge}\right)^{\nabla}$ with the filtration induced by the filtration on the $\mathbb{R}^{i}$. Then 13.19 .1 is given by the map in the filtered derived category

$$
\mathbb{R}^{\bullet}\left(U^{\wedge}\right)^{\nabla} \longrightarrow G C\left(\mathbb{R}^{\bullet}\left(\mathrm{B}_{\text {cris }}\left(U^{\wedge}\right)\right)\right) \stackrel{\simeq}{\longleftarrow} G C\left(\left(F \otimes J_{E}\right)\left(\mathrm{B}_{\text {cris }}\left(U^{\wedge}\right)\right)\right) .
$$

13.20. Combining all of this with 12.5 we obtain the transformation 13.17 .1 . By functoriality of the above construction and the fact that any two hypercovers as in 13.17 can be refined by a third, the map $\alpha$ is independent of the choices. The main comparison theorem, whose proof occupies the next section, is then the following: 
THEOREM 13.21. The transformation 13.17 .1 is an isomorphism compatible with Frobenius, Galois action, and strictly compatible with the filtrations. In addition 13.17 .1 is compatible with Chern classes of vector bundles on $X$.

\section{Proof of the comparison theorem}

14.1. Let us begin by proving that the transformation $\alpha$ in 13.17 .1 is compatible with Chern classes of vector bundles.

First consider the case of a line bundle $\mathscr{L}$ on $Y$. For the crystalline Chern class it is now necessary to recall the definition of the crystalline topos (as opposed to the convergent). For this let $\operatorname{Cris}\left(\left(Y, M_{Y}\right) / W\right)$ denote the site whose objects are strict closed immersions $\left(\left(U, M_{U}\right) \hookrightarrow\left(T, M_{T}\right)\right)$, where $U$ is an étale $Y$-scheme, together with a divided power structure $\gamma$ on the ideal of $U$ in $T$ such that the ideal of $U$ in $T$ is a nil-ideal. Morphisms and coverings are defined as in [29, 5.2], and the associated topos is denoted $\left(\left(Y, M_{Y}\right) / W\right)_{\text {cris }}$. Let $\mathscr{O}_{\left(Y, M_{Y}\right) \text { cris }}$ denote the structure sheaf sending $\left(U, M_{U}\right) \hookrightarrow\left(T, M_{T}\right)$ to $\Gamma\left(T, \mathscr{O}_{T}\right)$, and let $\mathscr{O}_{\left(Y, M_{Y}\right)}$ denote the sheaf $\left(\left(U, M_{U}\right) \hookrightarrow\left(T, M_{T}\right)\right) \mapsto \Gamma\left(U, \mathscr{O}_{U}\right)$. There is a natural surjection $\mathscr{O}_{\left(Y, M_{Y}\right) \text { cris }} \rightarrow$ $\mathscr{O}_{\left(Y, M_{Y}\right)}$ whose kernel $F^{1}$ is a PD-ideal. Looking at the units we get an exact sequence

$$
0 \rightarrow 1+F^{1} \rightarrow \mathscr{O}_{\left(Y, M_{Y}\right) \text { cris }}^{*} \rightarrow \mathscr{O}_{\left(Y, M_{Y}\right)}^{*} \rightarrow 0 .
$$

Since $F^{1}$ has divided powers there is also a logarithm map

$$
\log : 1+F^{1} \rightarrow \mathscr{O}_{\left(Y, M_{Y}\right) \text { cris }}, \quad 1+t \mapsto \sum_{m \geq 1}(-1)^{m-1}(m-1) ! t^{[m]} .
$$

Passing to cohomology we therefore get a map

$$
\begin{array}{rlc}
H^{1}\left(Y, \mathscr{O}_{Y}^{*}\right) & \underset{\log \otimes \mathbb{Q}}{\longrightarrow} & H^{2}\left(\left(\left(Y, M_{Y}\right) / W\right)_{\text {cris }}, 1+F^{1}\right) \\
& H^{2}\left(\left(\left(Y, M_{Y}\right) / W\right)_{\text {cris }}, \mathscr{O}_{\left(Y, M_{Y}\right) \text { cris }}\right) \otimes \mathbb{Q} \\
\simeq & H^{2}\left(\left(\left(Y, M_{Y}\right) / W\right)_{\text {conv }}, \mathscr{K}_{\left(Y, M_{Y}\right) / W}\right),
\end{array}
$$

where the last isomorphism is by [46, 3.1.1]. This defines the crystalline Chern $\operatorname{class} c_{1}^{\operatorname{cr}}(\mathscr{L})$.

To identify the image of $c_{1}^{\text {cr }}(\mathscr{L})$ under $\alpha$, assume that $\mathscr{L}$ is trivialized on each $U_{n}$ (after further refinement we may assume this is the case). Let $R_{n}$ denote the coordinate ring of $U_{n}$ so we have an exact sequence of simplicial groups

$$
0 \rightarrow 1+F_{\mathrm{A}_{\text {cris }}\left(U^{\wedge}\right)} \rightarrow \mathrm{A}_{\text {cris }}\left(U_{.}^{\wedge}\right)^{*} \rightarrow\left(\bar{R}_{.}^{\wedge} / p \bar{R}_{.}^{\wedge}\right)^{*} \rightarrow 0 .
$$

Choose trivializations of $\mathscr{L}$ on each $U_{n}$ so that the gluing data is given by an element $u \in\left(R_{1}^{\wedge} / p R_{1}^{\wedge}\right)^{*}$. By the method of 13.18 this defines a class in $H^{1}\left(G C\left(\left(\bar{R}_{.}^{\wedge} / p \bar{R}_{.}^{\wedge}\right)^{*}\right)\right)$ and hence by composing with the boundary map and the logarithm map we get $\alpha\left(c_{1}^{\mathrm{cr}}(\mathscr{L})\right)$ from the composite

$$
\begin{array}{rlc}
H^{1}\left(G C\left(\bar{R}_{.}^{\wedge} / p \bar{R}_{.}^{\wedge}\right)^{*}\right) & \rightarrow & \left.H^{2}\left(G C\left(1+F_{\mathrm{A}_{\text {cris }}(U \wedge}\right)\right)\right) \\
& \rightarrow & H^{2}\left(G C\left(\mathrm{~A}_{\text {cris }}\left(U^{\wedge}\right)\right)\right) \\
& \rightarrow & H^{2}\left(G C\left(\mathrm{~B}_{\text {cris }}\left(U^{\wedge}\right)\right)\right) .
\end{array}
$$

14.2. The image of the étale Chern class $c_{1}^{\text {et }}(\mathscr{L})$ in $H^{2}\left(G C\left(\mathrm{~B}_{\text {cris }} U^{\wedge}\right)\right)(1)$ under the isomorphism 12.5 is obtained as follows. Consider the sequence

$$
0 \rightarrow \mathbb{Z}_{p}(1) \rightarrow \lim _{\longleftarrow} \bar{R}^{\wedge *} \rightarrow \bar{R}^{\wedge *} \rightarrow 0,
$$


where the projective limit in the middle is given by the maps $\bar{R}^{\wedge *} \rightarrow \bar{R}^{\wedge *}$ sending $f$ to $f^{p}$. Then $c_{1}^{\text {et }}(\mathscr{L})$ is given by the map

$$
\begin{array}{rlc}
H^{1}\left(R^{\wedge *}\right) & \rightarrow & H^{1}\left(G C\left(\bar{R}^{\wedge *}\right)\right) \\
& \rightarrow & H^{2}\left(G C\left(\mathbb{Z}_{p}(1)\right)\right) \\
& \rightarrow & H^{2}\left(G C\left(\mathrm{~A}_{\text {cris }}\left(R_{.}^{\wedge}\right)\right)\right)(1) .
\end{array}
$$

Here the first map is induced by the maps $R_{m}^{\wedge *} \rightarrow G C\left(\bar{R}_{m}^{\wedge *}\right)$ obtained from the natural inclusion of $R_{m}^{\wedge *}$ into the Galois invariants in $\bar{R}_{m}^{\wedge *}$.

Let $\alpha: \mathbb{Z}_{p}(1) \rightarrow 1+F^{1}$ be the map defined in 11.1 .8 and let $\sigma: \lim _{\longleftarrow} \bar{R}^{\wedge *} \rightarrow$ $\mathrm{A}_{\text {cris }}\left(R^{\wedge}\right)$ be the composite

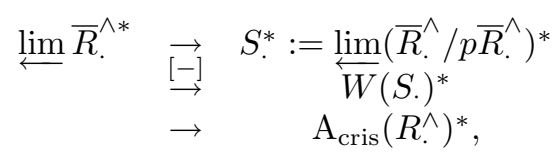

where $[-]: S^{*} \rightarrow W(S .)^{*}$ denote the Teichmuller lifting. Then it follows from the definitions that the diagram

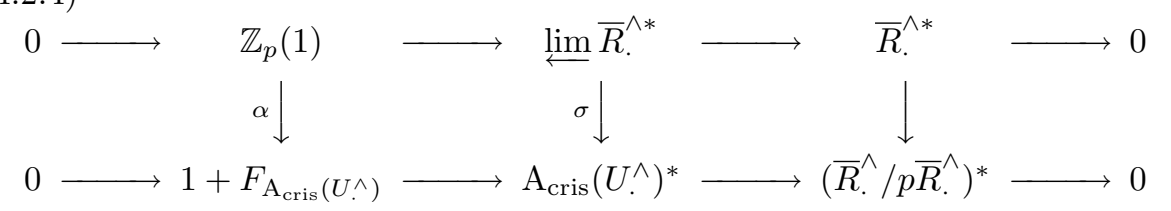

commutes, and from this one obtains the following:

Proposition 14.3. The diagram

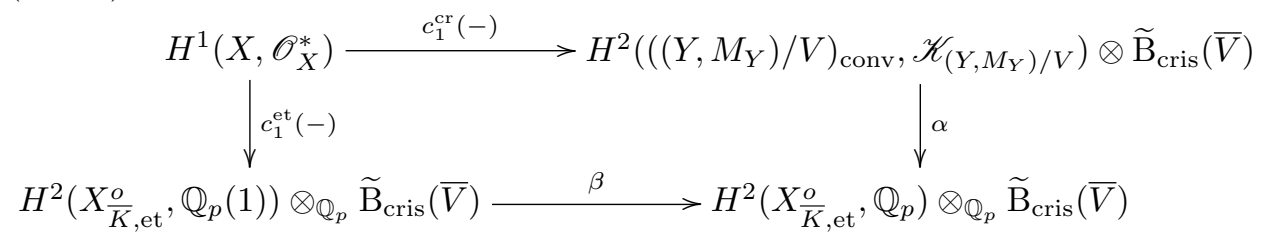

commutes, where the map $\beta$ is obtained from the map 11.1.9.

14.4. It is now an essentially formal consequence that $\alpha$ is also compatible with higher Chern classes of vector bundles. For this let $H^{*}$ denote either étale cohomology (with appropriate Tate twists) or crystalline cohomology and let $c_{1}$ : $H^{1}\left(X, \mathscr{O}_{X}^{*}\right) \rightarrow H^{2}(X)$ denote the Chern class map. Then for a vector bundle $\mathscr{E}$ on $X$ the higher Chern classes $c_{i}(\mathscr{E}) \in H^{2 i}(X)$ are defined as follows. Let $Y=\mathbb{P}_{X}\left(\mathscr{E}^{*}\right)$ denote the projective bundle associated to the dual of $\mathscr{E}$. Let $\xi \in H^{2}(Y)$ denote the first Chern class of the tautological line bundle on $Y$. Then the cohomology ring $H^{*}(Y)$ is a free $H^{*}(X)$-module with basis $1, \xi, \ldots, \xi^{r-1}$. In particular, there exists a unique relation

$$
\xi^{r}+c_{1}(\mathscr{E}) \xi^{r-1}+\cdots+c_{r-1}(\mathscr{E}) \xi+c_{r}(\mathscr{E})=0
$$

in $H^{2 r}(Y)$ with $c_{i}(\mathscr{E}) \in H^{2 i}(X)$. This defines the higher Chern classes of a vector bundle. Since the transformation $\alpha$ is functorial with respect to the map $Y \rightarrow X$ the following proposition follows from the case of a line bundle. 
Proposition 14.5. For any vector bundle $\mathscr{E}$ on $X$, we have

$$
\alpha\left(c_{i}^{\mathrm{cr}}(\mathscr{E})\right)=\beta^{\otimes i}\left(c_{i}^{\mathrm{et}}(\mathscr{E})\right),
$$

where $\beta^{\otimes i}$ is defined as in 11.1 .10 .

14.6. By construction the transformation $\alpha$ is functorial with respect to morphisms $f:\left(Y, D_{Y}\right) \rightarrow\left(X, D_{X}\right)$, where $X$ and $Y$ are smooth $V$-schemes of dimension $d_{Y}$ and $d_{X}$ respectively, $D_{Y} \subset Y$ and $D_{X} \subset X$ are divisors with normal crossings relative to $V$, and $f^{-1}\left(D_{X}\right) \subset D_{Y}$. For such a morphism $f$ the pullback functor $f^{*}$ has an adjoint $f_{*}$ with respect to the pairings provided by Poincaré duality. Precisely, for any decompositions $D_{X}=E \cup F$ and $D_{Y}=E_{Y} \cup F_{Y}$ such that $f^{-1}(E) \subset E_{Y}$ we get as in 8.20 maps

$f_{*}^{\text {cr }}: H^{i}\left(\left(\left(Y, M_{Y}\right) / V\right)_{\text {conv }}, f^{*} M \otimes J_{E_{Y}}\right) \rightarrow H^{2 d_{X}-2 d_{Y}+i}\left(\left(\left(X, M_{X}\right) / V\right)_{\text {conv }}, M \otimes J_{E}\right)$ and

$$
f_{*}^{\text {et }}: H_{E_{Y}, F_{Y}}^{i}\left(Y_{\bar{K}}^{o}, f^{*} L\right) \rightarrow H_{E, F}^{2 d_{X}-2 d_{Y}+i}\left(X_{\bar{K}}^{o}, L\right)\left(d_{X}-d_{Y}\right) .
$$

Proposition 14.7. Assume that $f$ is a closed immersion of pure codimension e. Then $\alpha \circ f_{*}^{\text {cr }}=\beta^{\otimes e} \circ f_{*}^{\text {et }} \circ \alpha$.

Proof. To ease notation write just

$$
\begin{gathered}
H_{\text {cris }}^{i}(X) \text { for } H^{i}\left(\left(\left(X, M_{X}\right) / V\right)_{\text {conv }}, M \otimes J_{E}\right), \\
H_{\text {cris }}^{i}(Y) \text { for } H^{i}\left(\left(\left(Y, M_{Y}\right) / V\right)_{\text {conv }}, f^{*} M \otimes J_{E_{Y}}\right), \\
H_{\text {et }}^{i}(X) \text { for } H_{E, F}^{i}\left(X \frac{o}{K}, L\right) \text { etc. }
\end{gathered}
$$

Let $Z$ denote the blow-up of $X \times \mathbb{P}^{1}$ along $Y \times\{\infty\}$. A calculation in local coordinates shows that the strict transform $W \subset Z$ of $Y \times \mathbb{P}^{1}$ maps isomorphically to $Y \times \mathbb{P}^{1}$ so we obtain a lifting $\tilde{f}: Y \times \mathbb{P}^{1} \hookrightarrow Z$. For any point $t \in \mathbb{P}^{1}$, let $Z_{t}$ denote the fiber of $Z$ over $t$ and let $f_{t}: Y \hookrightarrow Z_{t}$ be the inclusion induced by $\tilde{f}$. Let $\mathscr{N}$ denote the normal bundle of $Y$ in $X$. A calculation shows that $Z_{\infty}$ has two irreducible components $Z_{\infty}^{\prime}$ and $Z_{\infty}^{\prime \prime}$, where $Z_{\infty}^{\prime}$ is the projective bundle $\mathbb{P}_{Y}\left(\mathscr{N}^{*} \oplus \mathscr{O}_{Y}\right)$ and $Z_{\infty}^{\prime \prime}$ is the blow-up of $X$ along $Y$. Furthermore, the intersection $Z_{\infty}^{\prime} \cap Z_{\infty}^{\prime \prime}$ is the projective bundle $\mathbb{P}_{Y}\left(\mathscr{N}^{*}\right)$ sitting in $\mathbb{P}_{Y}\left(\mathscr{N}^{*} \oplus \mathscr{O}_{Y}\right)$ by the embedding given by the projection $\mathscr{N}^{*} \oplus \mathscr{O}_{Y} \rightarrow \mathscr{N}^{*}$ to the first factor. Finally the image of $Y$ in $Z_{\infty}$ is contained in $Z_{\infty}^{\prime}$ and does not meet $Z_{\infty}^{\prime} \cap Z_{\infty}^{\prime \prime}$.

Let $H^{*}(Z)$ denote the cohomology of $Z$ with partial compact support along the strict transform $\bar{D}$ of $D$, and let $H^{*}\left(Z, Z_{\infty}\right)$ denote the cohomology with compact support along $\bar{D} \cup Z_{\infty}$. If $j: Z-\left(\bar{D} \cup Z_{\infty}\right) \rightarrow Z-D$ and $i: Z_{\infty}-\left(\bar{D} \cap Z_{\infty}\right) \hookrightarrow Z-D$ are the inclusions, then for any sheaf $L$ on $Z_{\text {et }}$ there is an exact sequence of étale sheaves

$$
0 \rightarrow j ! j^{*} L \rightarrow L \rightarrow i_{*} i^{*} L \rightarrow 0 .
$$

It follows that the sequence

$$
H_{\mathrm{et}}^{*}\left(Z, Z_{\infty}\right) \stackrel{p}{\longrightarrow} H_{\mathrm{et}}^{*}(Z) \stackrel{q}{\longrightarrow} H_{\mathrm{et}}^{*}\left(Z_{\infty}\right)
$$

is exact. We also have a pullback map $j_{0}^{*}: H_{\mathrm{et}}^{*}(Z) \rightarrow H_{\mathrm{et}}^{*}\left(Z_{0}\right)$.

Lemma 14.8. The composite

$$
H_{\mathrm{et}}^{*}\left(Z, Z_{\infty}\right) \stackrel{p}{\longrightarrow} H_{\mathrm{et}}^{*}(Z) \stackrel{j_{0}^{*}}{\longrightarrow} H_{\mathrm{et}}^{*}\left(Z_{0}\right)
$$

is zero. 
Proof. The scheme $Z-\left(D \cup Z_{\infty}\right)$ is isomorphic to $(X-D) \times \mathbb{A}^{1}$ and hence by Künneth we have $H_{\mathrm{et}}^{*}\left(Z, Z_{\infty}\right) \simeq H_{\mathrm{et}}^{*}(X-D) \otimes H_{c}^{2}\left(\mathbb{A}^{1}\right) \simeq H_{\mathrm{et}}^{*-2}(X-D)(1)$. With this identification the map $p$ is given by the pushforward map

$$
j_{0 *}: H_{\mathrm{et}}^{*-2}(X-D)(1) \rightarrow H_{\mathrm{et}}^{*}(Z) .
$$

Thus we wish to show that the map

$$
j_{0}^{*} j_{0 *}: H_{\mathrm{et}}^{*-2}(X-D)(1) \rightarrow H_{\mathrm{et}}^{*}(X-D)
$$

is zero. This follows from the self-intersection formula [24, VII.4.1] which shows that this map is given by cup-product by the first Chern class of the conormal bundle of $Z_{0}$ in $Z$ which is trivial.

Note also that the map

$$
H_{\mathrm{et}}^{*}\left(Z_{\infty}^{\prime}\right) \rightarrow H_{\mathrm{et}}^{*}\left(Z_{\infty}^{\prime} \cap Z_{\infty}^{\prime \prime}\right)
$$

is surjective since both are generated over $H_{\mathrm{et}}^{*}(Y)$ by the first Chern class of the tautological line bundle. From the excision sequence we deduce that the map

$$
H_{\mathrm{et}}^{*}\left(Z_{\infty}\right) \rightarrow H_{\mathrm{et}}^{*}\left(Z_{\infty}^{\prime}\right) \oplus H_{\mathrm{et}}^{*}\left(Z_{\infty}^{\prime \prime}\right)
$$

is injective so we obtain an exact sequence

$$
H_{\mathrm{et}}^{*}\left(Z, Z_{\infty}\right) \stackrel{p}{\longrightarrow} H_{\mathrm{et}}^{*}(Z) \longrightarrow H_{\mathrm{et}}^{*}\left(Z_{\infty}^{\prime}\right) \oplus H_{\mathrm{et}}^{*}\left(Z_{\infty}^{\prime \prime}\right) .
$$

In either theory, the composite

$$
H^{*}(Y) \stackrel{\operatorname{pr}_{1}^{*}}{\longrightarrow} H^{*}\left(Y \times \mathbb{P}^{1}\right) \stackrel{\tilde{f}_{*}}{\longrightarrow} H^{*}(Z) \stackrel{j_{0}^{*}}{\longrightarrow} H^{*}\left(Z_{0}\right)=H^{*}(X)
$$

is equal to $f_{*}$. Combining this with 14.8 and 14.8.6 it follows that it suffices to show that for any element $a \in H_{\text {cris }}^{*}(Y)$ the images of the classes $\alpha\left(\tilde{f}_{*}^{\text {cr }}(a)\right)$ and $\tilde{f}_{*}^{\text {et }}(\alpha(a))$ in $H_{\mathrm{et}}^{*}\left(Z_{\infty}^{\prime}\right)$ and in $H_{\mathrm{et}}^{*}\left(Z_{\infty}^{\prime \prime}\right)$ are equal. In either theory the formation of pushforwards commutes with pullbacks along smooth subvarieties transverse to the boundary. In the case of a divisor transverse to the boundary, this follows from the description of the pushforward as a boundary map 8.23 and its crystalline analog (which we leave to the reader). The general case is deduced from this using the trick following 8.54 in the proof of 8.21 . This reduces the proof of 14.7 to the case of $Y \hookrightarrow Z_{\infty}^{\prime}$ (note that the case of $Z_{\infty}^{\prime \prime}$ is trivial since $Y \times \mathbb{P}^{1} \cap Z_{\infty}^{\prime \prime}$ is empty).

The proof is now reduced to the case of the inclusion $f: Y \hookrightarrow \mathbb{P}_{Y}\left(\mathscr{N}^{*} \oplus \mathscr{O}_{Y}\right)$. For any class $\beta \in H^{*}(Y)$ we have $f_{*}(\beta)=f_{*}\left(f^{*} p^{*} \beta\right)=f_{*}(1) \cup p^{*} \beta$, and hence it suffices to consider the case of constant coefficients and $f_{*}(1)$. But in this case it is well-known that $f_{*}(1)$ in either theory is given by $c_{r}\left(\mathscr{N}^{*}\right)$ (this can for instance be verified in the rational Chow ring where it is standard). By 14.5 this completes the proof of 14.7

Corollary 14.9. Let $\operatorname{tr}^{\mathrm{cr}}: H^{2 d}\left(\left(\left(X, M_{X}\right) / V\right)_{\text {conv }}, M \otimes J_{D}\right) \otimes_{K} \widetilde{\mathrm{B}}_{\text {cris }}(\bar{V}) \rightarrow$ $\widetilde{\mathrm{B}}_{\text {cris }}(\bar{V})$ denote the crystalline trace map and $\operatorname{tr}^{\mathrm{et}}: H_{c}^{2 d}\left(X_{\bar{K}}^{o}, \mathbb{Q}_{p}\right) \otimes_{\mathbb{Q}_{p}} \widetilde{\mathrm{B}}_{\text {cris }}(\bar{V}) \rightarrow$ $\widetilde{\mathrm{B}}_{\text {cris }}(\bar{V})(d)$ the étale trace map. Then the diagram

$$
\begin{array}{ccc}
H^{2 d}\left(\left(\left(X, M_{X}\right) / V\right)_{\text {conv }}, M \otimes J_{D}\right) \otimes_{K} \widetilde{\mathrm{B}}_{\text {cris }}(\bar{V}) \stackrel{\operatorname{tr}^{\text {cr }}}{\longrightarrow} & \widetilde{\mathrm{B}}_{\text {cris }}(\bar{V}) \\
\alpha \downarrow & \beta^{\otimes d} \uparrow \\
H_{c}^{2 d}\left(X_{\bar{K}}^{o}, \mathbb{Q}_{p}\right) \otimes_{\mathbb{Q}_{p}} \widetilde{\mathrm{B}}_{\text {cris }}(\bar{V}) & \stackrel{\operatorname{tr}^{\mathrm{et}}}{\longrightarrow} \widetilde{\mathrm{B}}_{\text {cris }}(\bar{V})(d)
\end{array}
$$


commutes. In particular, the map

$$
\alpha: H^{2 d}\left(\left(\left(X, M_{X}\right) / V\right)_{\text {conv }}, M \otimes J_{D}\right) \otimes_{K} \widetilde{\mathrm{B}}_{\text {cris }}(\bar{V}) \rightarrow H_{c}^{2 d}\left(X_{\bar{K}}^{o}, \mathbb{Q}_{p}\right) \otimes_{\mathbb{Q}_{p}} \widetilde{\mathrm{B}}_{\text {cris }}(\bar{V})
$$

is an isomorphism.

Proof. It suffices to show this after making an extension of $V$ when $X^{o} \rightarrow$ $\operatorname{Spec}(V)$ has a section. In this case the corollary follows from observing that the trace map is characterized by the fact that it sends the class of a point in $X^{o}$ to 1.

14.10. It follows that there exists a unique map

$$
\alpha^{t}: H_{E, F}^{*}(X \bar{K}, L) \otimes_{\mathbb{Q}_{p}} \widetilde{\mathrm{B}}_{\text {cris }}(\bar{V}) \rightarrow H^{*}\left(\left(\left(X, M_{X}\right) / V\right)_{\text {conv }}, M \otimes J_{E}\right) \otimes_{K} \widetilde{\mathrm{B}}_{\text {cris }}(\bar{V})
$$

such that for any element

$$
a \in H^{2 d-*}\left(\left(\left(X, M_{X}\right) / V\right)_{\mathrm{conv}}, M \otimes J_{F}\right)
$$

we have

$$
\operatorname{tr}^{\mathrm{cr}}\left(\alpha^{t}(b) \cup a\right)=\beta^{\otimes d}\left(\operatorname{tr}^{\mathrm{et}}(b \cup \alpha(a))\right) .
$$

Using the same argument used in 8.60 and 8.61 one shows that $\alpha^{t}$ is also an isometry. It follows that $\alpha$ and $\alpha^{t}$ are both isomorphisms. This completes the proof that 13.17.1 is an isomorphism.

14.11. Finally let us verify that the isomorphism $\alpha$ is strictly compatible with the filtrations. For this it suffices to show that the morphism on the associated graded is an inclusion, for then the inverse for $\alpha$ also preserves the filtrations.

Let $D=E \cup F$ be a decomposition of the boundary, and let $F^{\bullet}$ denote the filtration on $H^{*}\left(X / K, M \otimes J_{E}\right)$ induced by the filtration on $M$ and let $\widehat{F}^{\bullet}$ denote the filtration on $H^{*}\left(X / K, M^{*} \otimes J_{F}\right)$ induced by the dual filtration on $M^{*}$. It then suffices to show that the induced pairing

$$
\operatorname{gr}_{F}^{s} H^{k}\left(X / K, M \otimes J_{E}\right) \otimes \operatorname{gr}_{\widehat{F}}^{-s} H^{2 d-k}\left(X / K, M^{*} \otimes J_{F}\right) \rightarrow H^{d}\left(X, \Omega_{X}^{d}\right)
$$

is a perfect pairing for all $k$ and $s$. To see that this suffices let $G$ denote the filtration on $H_{E, F}^{*}\left(X \frac{o}{K}, L\right) \otimes_{\mathbb{Q}_{p}} \widetilde{\mathrm{B}}_{\text {cris }}(\bar{V})$ induced by the filtration on $\widetilde{\mathrm{B}}_{\text {cris }}(\bar{V})$, and to ease the notation write

$$
\begin{aligned}
\operatorname{gr}_{F}^{s, k} & :=\operatorname{gr}_{F}^{s} H^{k}\left(X / K, M \otimes J_{E}\right) \otimes_{K} \widetilde{\mathrm{B}}_{\mathrm{cris}}(\bar{V}), \\
\operatorname{gr}_{\widehat{F}}^{-s, 2 d-k} & :=\operatorname{gr}_{\widehat{F}}^{-s} H^{2 d-k}\left(X / K, M^{*} \otimes J_{F}\right) \otimes_{K} \widetilde{\mathrm{B}}_{\text {cris }}(\bar{V}),
\end{aligned}
$$

and

$$
\operatorname{gr}_{G}^{s, k}:=\operatorname{gr}_{G}^{s} H_{E, F}^{k}\left(X \bar{K}^{o}, L\right) \otimes_{\mathbb{Q}_{p}} \widetilde{\mathrm{B}}_{\text {cris }}(\bar{V}) .
$$

We then have a commutative diagram

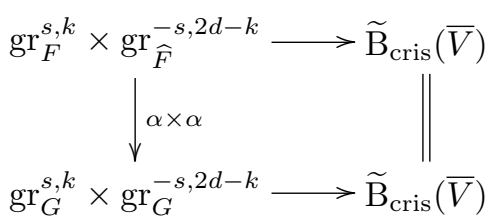

and since both the top and bottom lines are perfect pairings it follows that the map

$$
\operatorname{gr}_{F}^{s} H^{k}\left(X / K, M \otimes J_{E}\right) \otimes_{K} \widetilde{\mathrm{B}}_{\text {cris }}(\bar{V}) \rightarrow \operatorname{gr}_{G}^{s} H_{E, F}^{k}\left(X \bar{K}_{\bar{K}}^{o}, L\right) \otimes_{\mathbb{Q}_{p}} \widetilde{\mathrm{B}}_{\text {cris }}(\bar{V})
$$

is an inclusion. 
The following therefore completes the proof of 13.21

LEMMA 14.12. The pairing 14.11.1 is perfect.

Proof. For every integer $i$ there is a natural inclusion $(14.12 .1)$

$\widehat{F}^{-i} \subset\left\{c \in H^{2 d-k}\left(X / K, M^{*} \otimes J_{F}\right) \mid c \cup \beta=0\right.$ for all $\left.\beta \in F^{i} H^{k}\left(X / K, M \otimes J_{E}\right)\right\}$.

To prove the lemma it suffices to show that these inclusions are isomorphisms. Let $\mathcal{F}^{i} \otimes J_{E}$ denote the complex

$$
\mathrm{Fil}_{\mathcal{M}}^{i} \otimes J_{E} \rightarrow \mathrm{Fil}_{\mathcal{M}}^{i-1} \otimes J_{E} \otimes \Omega_{\left(X_{K}, M_{X_{K}}\right) / K}^{1} \rightarrow \cdots
$$

and let $\operatorname{DR}\left(\mathcal{M} \otimes J_{E}\right) / \mathcal{F}^{i}$ denote the quotient of the de Rham complex of $(\mathcal{M}, \nabla)$ tensored with the ideal $J_{E}$ by the subcomplex $\mathcal{F}_{i} \otimes J_{E}$. The subspace $F^{i}$ of $H^{k}\left(X / K, M \otimes J_{E}\right)$ is defined to be the image of the natural map

$$
H^{k}\left(X_{K}, \mathcal{F}^{i} \otimes J_{E}\right) \rightarrow H^{k}\left(X_{K}, D R\left(\mathcal{M} \otimes J_{E}\right)\right),
$$

and $\widehat{F}^{-i} \subset H^{2 d-k}\left(X_{K}, D R\left(\mathcal{M}^{*} \otimes J_{F}\right)\right)$ is the image of the natural map

$$
H^{2 d-k}\left(X_{K}, \widehat{\mathcal{F}}^{-i} \otimes J_{F}\right) \rightarrow H^{2 d-k}\left(X_{K}, D R\left(\mathcal{M}^{*} \otimes J_{F}\right)\right),
$$

One shows as in 13.7 that the natural pairing

$$
H^{k}\left(X_{K}, \mathcal{F}^{s} \otimes J_{E}\right) \times H^{2 d-k}\left(X_{K}, D R\left(\mathcal{M}^{*} \otimes J_{F}\right) / \widehat{F}^{-s} \otimes J_{F}\right) \rightarrow H^{d}\left(X_{K}, \Omega_{X_{K}}^{d}\right)
$$

is a perfect pairing, and from this it follows that the image of 14.12 .4 is via Poincaré duality identified with the elements annihilating the image of 14.12 .3 .

\section{From $\widetilde{\mathrm{B}}_{\text {cris }}(\bar{V})$ to $\mathrm{B}_{\text {cris }}(\bar{V})$}

In this section we explain how to lift the isomorphism 13.17.1 to an isomorphism over $\mathrm{B}_{\text {cris }}(\bar{V})$. This will be done using some general observations about the rings $\mathrm{B}_{\mathrm{dR}}, \widetilde{\mathrm{B}}_{\mathrm{cris}}(\bar{V})$, and $\mathrm{B}_{\text {cris }}(\bar{V})$. Let us start by recalling the definitions of these rings.

15.1. Let $V$ be a complete discrete valuation ring of mixed characteristic $(0, p)$, field of fractions $K$, and perfect residue field $k$. Let $W$ be the ring of Witt vectors of $k$, and let $K_{0} \subset K$ be the field of fractions of $W$. Let $K \hookrightarrow \bar{K}$ be an algebraic closure, and let $S_{\bar{V}}$ (resp. $\left.\mathrm{A}_{\text {cris }}(\bar{V}), \mathrm{B}_{\text {cris }}(\bar{V}), \widetilde{\mathrm{B}}_{\text {cris }}(\bar{V})\right)$ be the rings defined in 11.1 . As in 11.1 .2 there is a surjection

$$
\theta: W\left(S_{\bar{V}}\right) \rightarrow \bar{V}^{\wedge},
$$

where $\bar{V}^{\wedge}$ denotes the $p$-adic completion of $\bar{V}$. If $J$ denotes the kernel of $\theta$ then $\mathrm{A}_{\text {cris }}(\bar{V})$ is defined to be the $p$-adic completion of the divided power envelope $D_{J}\left(W\left(S_{\bar{V}}\right)\right)$.

Let $t \in \mathrm{A}_{\text {cris }}(\bar{V})$ be the element 11.1 .5 so that $\mathrm{B}_{\text {cris }}(\bar{V})$ is equal to the localization $\mathrm{A}_{\text {cris }}(\bar{V})[1 / t]$. Also for $\alpha \in \Lambda_{+}:=\mathbb{Z}[1 / p] \cap \mathbb{Q}_{>0}$ define $\delta_{\alpha} \in \mathrm{A}_{\text {cris }}(\bar{V})$ as in 11.12 so that $\widetilde{\mathrm{B}}_{\text {cris }}(\bar{V})=\mathrm{B}_{\text {cris }}(\bar{V})\left[1 / \delta_{\alpha}\right]_{\alpha \in \Lambda_{+}}$. Note that by construction $\delta_{1 / p^{n}}^{p^{n}}=\delta_{1}$ so we also have $\widetilde{\mathrm{B}}_{\text {cris }}(\bar{V})=\mathrm{B}_{\text {cris }}(\bar{V})\left[1 / \delta_{1}\right]$. 
15.2. Let us also recall the rings $B_{d R}^{+}$and its field of fractions $B_{d R}$. Let $\left(B_{n}, J_{n},[]\right)$ be the divided power ring which is the reduction of $\mathrm{A}_{\text {cris }}(\bar{V})$ modulo $p^{n}$ (for a cohomological interpretation of this ring see 49, A1.6 and paragraph following A2.10]). Then we set

$$
\mathrm{B}_{\mathrm{dR}}^{+}:={\underset{l}{r}}_{\lim }(\mathbb{Q} \otimes \underbrace{\lim }_{n} B_{n} / J_{n}^{[r]}) .
$$

There is a natural map $\mathrm{A}_{\text {cris }}(\bar{V}) \otimes \mathbb{Q} \rightarrow \mathrm{B}_{\mathrm{dR}}^{+}$, and one can show (see for example [16, 1.5.2]) that $\mathrm{B}_{\mathrm{dR}}^{+}$is a complete discrete valuation ring and that the image of $t \in \mathrm{A}_{\text {cris }}(\bar{V})$ in $\mathrm{B}_{\mathrm{dR}}^{+}$is a uniformizer. The field $\mathrm{B}_{\mathrm{dR}}$ is defined to be the field of fractions of $\mathrm{B}_{\mathrm{dR}}^{+}$. There is a natural inclusion $\mathrm{B}_{\text {cris }}(\bar{V}) \hookrightarrow \mathrm{B}_{\mathrm{dR}}$, which induces an inclusion $\widetilde{\mathrm{B}}_{\text {cris }}(\bar{V}) \hookrightarrow \mathrm{B}_{\mathrm{dR}}$. Note that the action of $G_{K}$ on $\mathrm{A}_{\text {cris }}(\bar{V})$ induces an action of $G_{K}$ on $\mathrm{B}_{\mathrm{dR}}^{+}$and $\mathrm{B}_{\mathrm{dR}}$ and the inclusion $\widetilde{\mathrm{B}}_{\text {cris }}(\bar{V}) \hookrightarrow \mathrm{B}_{\mathrm{dR}}$ is compatible with the $G_{K}$-actions.

LEMma 15.3. (i) The natural map

$$
K_{0} \simeq \mathrm{B}_{\text {cris }}(\bar{V})^{G_{K}} \rightarrow \widetilde{\mathrm{B}}_{\text {cris }}(\bar{V})^{G_{K}}
$$

is an isomorphism, where the first isomorphism is by [17, 5.1.2].

(ii) For any $G_{K}$-representation $W$ the natural map

$$
K \otimes_{K_{0}} \widetilde{\mathrm{B}}_{\text {cris }}(\bar{V}) \otimes_{\mathbb{Q}_{p}} W \rightarrow \mathrm{B}_{\mathrm{dR}} \otimes_{\mathbb{Q}_{p}} W
$$

is injective.

Proof. By [16, 1.5.7] we have $K \simeq \mathrm{B}_{\mathrm{dR}}^{G_{K}}$, so $\widetilde{\mathrm{B}}_{\text {cris }}(\bar{V})^{G_{K}}$ is a subring of $K$ containing $K_{0}$. Also by [16, 4.2.4] the natural map

$$
K \otimes_{K_{0}} \mathrm{~B}_{\text {cris }}(\bar{V}) \rightarrow \mathrm{B}_{\mathrm{dR}}
$$

is injective. Since localization is an exact functor this implies that the natural map

$$
K \otimes_{K_{0}} \widetilde{\mathrm{B}}_{\text {cris }}(\bar{V}) \rightarrow \mathrm{B}_{\mathrm{dR}}
$$

is also injective, which implies that the map

$$
K \otimes_{K_{0}} \widetilde{\mathrm{B}}_{\text {cris }}(\bar{V})^{G_{K}} \rightarrow \mathrm{B}_{\mathrm{dR}}^{G_{K}}=K
$$

is an inclusion. This is only possible if $\widetilde{\mathrm{B}}_{\text {cris }}(\bar{V})^{G_{K}}=K_{0}$ and we obtain (i).

For (ii) note that it suffices to consider $W=\mathbb{Q}_{p}$, in which case we already remarked the result (namely that 15.3 .4 is injective).

15.4. For any finite dimensional continuous $G_{K}$-representation $W$ set

$$
\begin{gathered}
D_{\text {cris }}(W):=\left(W \otimes_{\mathbb{Q}_{p}} \widetilde{\mathrm{B}}_{\text {cris }}(\bar{V})\right)^{G_{K}}, \\
D_{\text {cris }}(W):=\left(W \otimes_{\mathbb{Q}_{p}} \mathrm{~B}_{\text {cris }}(\bar{V})\right)^{G_{K}}, \\
D_{\mathrm{dR}}(W):=\left(W \otimes_{\mathbb{Q}_{p}} \mathrm{~B}_{\mathrm{dR}}\right)^{G_{K}} .
\end{gathered}
$$

There are natural inclusions

$$
D_{\text {cris }}(W) \subset D_{\text {cris }}(W) \subset D_{\mathrm{dR}}(W) .
$$

Since $D_{\mathrm{dR}}(W)$ is a finite dimensional $K$-vector space of dimension $\leq \operatorname{dim}_{\mathbb{Q}_{p}} W[\mathbf{1 7}$, 1.4.2 Proposition] and the natural map

$$
K \otimes_{K_{0}} D_{\text {cris }}(W) \rightarrow D_{\mathrm{dR}}(W)
$$


is injective (this follows from 15.3 (ii) by taking $G_{K}$-invariants), the $K_{0}$-vector space $D_{\text {cris }}(W)$ has dimension $\leq \operatorname{dim}_{\mathbb{Q}_{p}}(W)$. Moreover if

$$
\operatorname{dim}_{K_{0}} D_{\text {cris }}(W)=\operatorname{dim}_{\mathbb{Q}_{p}} W
$$

then the map 15.4 .5 is an isomorphism.

There is also a commutative diagram

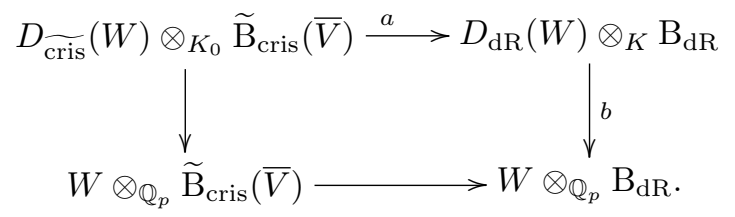

The map $a$ is an inclusion as it is equal to the composite of the inclusion

$$
D_{\text {cris }}(W) \otimes_{K_{0}} \widetilde{\mathrm{B}}_{\text {cris }}(\bar{V}) \hookrightarrow D_{\text {cris }}(W) \otimes_{K_{0}} \mathrm{~B}_{\mathrm{dR}}=\left(D_{\text {cris }}(W) \otimes_{K_{0}} K\right) \otimes_{K} \mathrm{~B}_{\mathrm{dR}}
$$

and the inclusion

$$
\left(D_{\text {cris }}(W) \otimes_{K_{0}} K\right) \otimes_{K} \mathrm{~B}_{\mathrm{dR}} \hookrightarrow D_{\mathrm{dR}}(W) \otimes_{K} \mathrm{~B}_{\mathrm{dR}}
$$

obtained from 15.4.5. The map $b$ is an injection by [17, 3.6 Proposition]. It follows that the natural map

$$
D_{\text {cris }}(W) \otimes_{K_{0}} \widetilde{\mathrm{B}}_{\text {cris }}(\bar{V}) \rightarrow W \otimes_{\mathbb{Q}_{p}} \widetilde{\mathrm{B}}_{\text {cris }}(\bar{V})
$$

is also an injection. We say that $W$ is $\widetilde{\mathrm{B}}_{\text {cris }}(\bar{V})$-admissible if this map 15.4 .10 is an isomorphism.

Similarly one obtains a notion of $\mathrm{B}_{\text {cris }}(\bar{V})$-admissible (resp. $\mathrm{B}_{\mathrm{dR}}$-admissible) representations. Following standard terminology we call a $\mathrm{B}_{\text {cris }}(\bar{V})$-admissible (resp. $\mathrm{B}_{\mathrm{dR}}$-admissible) representation a crystalline (resp. de Rham) representation. Note that we have the following implications:

$$
\text { crystalline } \Longrightarrow \widetilde{\mathrm{B}}_{\text {cris }}(\bar{V}) \text {-admissible } \Longrightarrow \text { de Rham. }
$$

The main result of this section is the following:

TheOREM 15.5. Any $\widetilde{\mathrm{B}}_{\text {cris }}(\bar{V})$-admissible representation is crystalline.

Proof. Let $W$ be a $\widetilde{\mathrm{B}}_{\text {cris }}(\bar{V})$-admissible representation and let $M_{0}$ denote $D_{\text {cris }}(W)$ so we have a $G_{K}$-equivariant isomorphism

$$
W \otimes_{\mathbb{Q}_{p}} \widetilde{\mathrm{B}}_{\text {cris }}(\bar{V}) \simeq M_{0} \otimes_{K_{0}} \widetilde{\mathrm{B}}_{\text {cris }}(\bar{V}) .
$$

Note that then the natural map

$$
M_{0} \rightarrow W \otimes_{\mathbb{Q}_{p}} \widetilde{\mathrm{B}}_{\text {cris }}(\bar{V}) \hookrightarrow W \otimes_{\mathbb{Q}_{p}} \mathrm{~B}_{\mathrm{dR}}
$$

induces an isomorphism

$$
M:=M_{0} \otimes_{K_{0}} K \rightarrow\left(W \otimes_{\mathbb{Q}_{p}} \mathrm{~B}_{\mathrm{dR}}\right)^{G_{K}} .
$$

Let $\pi \in V$ be a uniformizer and fix a sequence of elements $s=\left\{s_{n}\right\}_{n \geq 0}$ of $\bar{V}$ such that $s_{0}=\pi, s_{n+1}^{p}=s_{n}$. Denote by $\epsilon(s) \in W\left(S_{\bar{V}}\right)$ the Teichmuller lifting of the element of $S_{\bar{V}}$ defined by the reductions of the $s_{n}$. Then one can show (see for example [30, 2.2]) that the series $\log \left(\epsilon(s) \pi^{-1}\right)$ converges to an element $u_{s} \in \mathrm{B}_{\mathrm{dR}}^{+}$. Let $\mathrm{B}_{\mathrm{st}} \subset \mathrm{B}_{\mathrm{dR}}$ denote the subalgebra over $\mathrm{B}_{\text {cris }}(\bar{V})$ generated by $u_{s}$. Then as explained in $[\mathbf{1 6}, 3.1 .5]$ the element $u_{s}$ is transcendental over $\mathrm{B}_{\text {cris }}(\bar{V})$ so 
$\mathrm{B}_{\text {st }}$ is isomorphic to a polynomial algebra over $\mathrm{B}_{\text {cris }}(\bar{V})$. Also the $G_{K}$-action on $\mathrm{B}_{\mathrm{dR}}$ restricts to a $G_{K}$-action on $\mathrm{B}_{\mathrm{st}}$.

LEMMA 15.6. We have

$$
\mathrm{B}_{\text {cris }}(\bar{V})=\widetilde{\mathrm{B}}_{\text {cris }}(\bar{V}) \cap \mathrm{B}_{\mathrm{st}} \subset \mathrm{B}_{\mathrm{dR}} .
$$

Proof. Since localization is an exact functor, the subalgebra of $\mathrm{B}_{\mathrm{dR}}$ generated by $\widetilde{\mathrm{B}}_{\text {cris }}(\bar{V})$ and $u_{s}$ is isomorphic to a polynomial algebra $\widetilde{\mathrm{B}}_{\text {cris }}(\bar{V})[u]$ such that a polynomial $\sum_{i} a_{i} u^{i}$ is in $\mathrm{B}_{\mathrm{st}}=\mathrm{B}_{\text {cris }}(\bar{V})[u]$ if and only if all the coefficients $a_{i}$ are in $\mathrm{B}_{\text {cris }}(\bar{V})$. This implies the lemma.

From this we deduce 15.5 as follows. By [3, 0.7] any de Rham representation is potentially semistable which implies that there exists a finite extension $K \subset K^{\prime} \subset$ $\bar{K}$ such that

$$
K^{\prime} \otimes_{K_{0}^{\prime}}\left(W \otimes_{\mathbb{Q}_{p}} \mathrm{~B}_{\mathrm{st}}\right)^{G_{K^{\prime}}} \rightarrow\left(W \otimes_{\mathbb{Q}_{p}} \mathrm{~B}_{\mathrm{dR}}\right)^{G_{K^{\prime}}}
$$

is an isomorphism, where $K_{0}^{\prime}$ denotes the field of fractions of the ring of Witt vectors of the residue field of $K^{\prime}$. Let $\widetilde{\mathrm{B}}_{\text {st }}$ denote $\mathrm{B}_{\mathrm{st}} \otimes_{\mathrm{B}_{\text {cris }}(\bar{V})} \widetilde{\mathrm{B}}_{\text {cris }}(\bar{V})$. By a similar reasoning as in 15.3 we have $\widetilde{\mathrm{B}}_{\mathrm{st}}^{G_{K^{\prime}}}=K_{0}^{\prime}$. It follows that we have a diagram of injections of $K_{0}^{\prime}$-vector spaces of dimension equal to $\operatorname{dim}_{\mathbb{Q}_{p}} W$

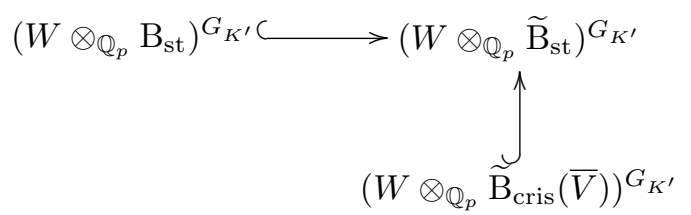

It follows that all these maps are isomorphisms. Therefore the image of the map

$$
M_{0} \rightarrow W \otimes_{\mathbb{Q}_{p}} \widetilde{\mathrm{B}}_{\mathrm{st}}
$$

is contained in

$$
\left(W \otimes_{\mathbb{Q}_{p}} \widetilde{\mathrm{B}}_{\text {cris }}(\bar{V})\right) \cap\left(W \otimes_{\mathbb{Q}_{p}} \mathrm{~B}_{\mathrm{st}}\right)
$$

which by 15.6 is equal to $W \otimes_{\mathbb{Q}_{p}} \mathrm{~B}_{\text {cris }}(\bar{V})$. Therefore $M_{0} \subset D_{\text {cris }}(W)$ which implies that

$$
\operatorname{dim}_{K_{0}} D_{\text {cris }}(W)=\operatorname{dim}_{\mathbb{Q}_{p}} W .
$$

Therefore $W$ is crystalline [17, 1.4.2 Proposition].

REMARK 15.7. It should be remarked that Berger's theorem that de Rham representations are potentially semistable was not available at the time of Tsuji's original proof of the comparison theorem.

15.8. Note that if $W$ is a crystalline representation, then the natural map $D_{\text {cris }}(W) \rightarrow \widetilde{D_{\text {cris }}}(W)$ is an isomorphism and the isomorphism

$$
D_{\text {cris }}(W) \otimes_{K_{0}} \widetilde{\mathrm{B}}_{\text {cris }}(\bar{V}) \simeq W \otimes_{\mathbb{Q}_{p}} \widetilde{\mathrm{B}}_{\text {cris }}(\bar{V})
$$

is induced by the isomorphism

$$
D_{\text {cris }}(W) \otimes_{K_{0}} \mathrm{~B}_{\text {cris }}(\bar{V})=D_{\text {cris }}(W) \otimes_{K_{0}} \mathrm{~B}_{\text {cris }}(\bar{V}) \simeq W \otimes_{\mathbb{Q}_{p}} \mathrm{~B}_{\text {cris }}(\bar{V}) .
$$


Corollary 15.9. The map $\alpha$ in 13.17.1 induces an isomorphism (15.9.1) $\mathrm{B}_{\text {cris }}(\bar{V}) \otimes_{K} H^{*}\left(\left(\left(Y, M_{Y}\right) / V\right)_{\text {conv }}, F \otimes J_{E}\right) \rightarrow \mathrm{B}_{\text {cris }}(\bar{V}) \otimes_{\mathbb{Q}_{p}} H_{E, F}^{*}\left(X_{\bar{J}, \text { et }}, L\right)$ compatible with Galois actions, Frobenius, and filtrations.

\section{Appendix A. Künneth formula in group cohomology}

The results of this appendix are standard, but we were unable to locate a suitable reference so we include them here.

A.1. Let $R$ be a ring and let $G$ and $H$ be profinite groups. For a continuous representation $M$ (resp. $N$ ) of $G$ (resp. $H$ ) over $R$, let $M \otimes N$ be the continuous representation of $G \times H$ over $R$ with underlying $R$-module $M \otimes_{R} N$ and action of $(g, h) \in G \times H$ given by

$$
m \otimes n \mapsto(g \cdot m) \otimes(h \cdot n) .
$$

A.2. Let $\Delta$ denote the standard simplicial category with objects the finite ordered sets $[n]=\{0, \ldots, n\}$ and morphisms the order preserving maps. Let $G^{[n]}$ denote the set of functions $[n] \rightarrow G^{[n]}$, and let

$$
\operatorname{Hom}_{G}^{\mathrm{cts}}\left(G^{[n]}, M\right)
$$

denote the set of continuous functions $\varphi: G^{[n]} \rightarrow M$ (where $M$ has the discrete topology) such that for every $g \in G$ and $\left(g_{0}, \ldots, g_{n}\right) \in G^{[n]}$ we have

$$
\varphi\left(g g_{0}, \ldots, g g_{n}\right)=g \cdot \varphi\left(g_{0}, \ldots, g_{n}\right) .
$$

For any morphism $\delta:[m] \rightarrow[n]$ in $\Delta$ there is an induced morphism

$$
\operatorname{Hom}_{G}^{\mathrm{cts}}\left(G^{[m]}, M\right) \rightarrow \operatorname{Hom}_{G}^{\mathrm{cts}}\left(G^{[n]}, M\right)
$$

sending $\varphi: G^{[m]} \rightarrow M$ to the function $G^{[n]} \rightarrow M$ sending

$$
\left(g_{0}, \ldots, g_{n}\right) \mapsto \varphi\left(g_{\delta(0)}, \ldots, g_{\delta(m)}\right) .
$$

In this way we get a cosimplicial $R$-module

$$
A_{G}:=\operatorname{Hom}_{G}^{\mathrm{cts}}\left(G^{[-]}, M\right)
$$

and as is well-known the corresponding total complex $\widetilde{A}_{G}$ computes $R \Gamma(G, M)$ (see for example [44, I $\S 2.2])$.

Similarly, $R \Gamma(H, N)$ and $R \Gamma(G \times H, M \otimes N)$ are computed by the total complexes $\widetilde{A}_{H}$ and $\widetilde{A}_{G \times H}$ respectively of the cosimplicial $R$-modules

$$
A_{H}:=\operatorname{Hom}_{H}^{\mathrm{cts}}\left(H^{[-]}, N\right) \text { and } A_{G \times H}:=\operatorname{Hom}_{G \times H}^{\mathrm{cts}}\left((G \times H)^{[-]}, M \otimes N\right) .
$$

A.3. Now in general (see for example [28, §I.1.2]) if $A$ and $B$ are cosimplicial $R$-modules, then we obtain a co-bisimplicial $R$-module $A \otimes B$ (i.e. functor $\Delta^{2} \rightarrow$ $\operatorname{Mod}_{R}$ ) by the formula

$$
([n],[m]) \mapsto A_{n} \otimes_{R} B_{m},
$$

whose associated total complex $\widetilde{A \otimes B}$ is equal to $\widetilde{A} \otimes_{R} \widetilde{B}$.

Let $A \otimes^{\Delta} B$ denote the restriction of $A \otimes B$ to the diagonal $\Delta \subset \Delta^{2}$, so $A \otimes^{\Delta} B$ is the cosimplicial $R$-module

$$
[n] \mapsto A_{n} \otimes B_{n}
$$

By the Eilenberg-Zilber theorem [28, I.1.2.2] there is a natural quasi-isomorphism

$$
\widetilde{A \otimes B} \rightarrow \widetilde{A \otimes^{\Delta} B}
$$


A.4. For any $[n] \in \Delta$ there is a natural map

$$
\operatorname{Hom}_{G}^{\mathrm{cts}}\left(G^{[n]}, M\right) \otimes_{R} \operatorname{Hom}_{H}^{\mathrm{cts}}\left(H^{[n]}, N\right) \rightarrow \operatorname{Hom}_{G \times H}^{\mathrm{cts}}\left((G \times H)^{[n]}, M \otimes N\right),
$$

sending

$$
\varphi \otimes \psi \mapsto\left(\left(g_{0}, h_{0}\right), \ldots,\left(g_{n}, h_{n}\right)\right) \mapsto \varphi\left(g_{0}, \ldots, g_{n}\right) \otimes \psi\left(h_{0}, \ldots, h_{n}\right) .
$$

A straightforward verification shows that this is compatible with the cosimplicial structures and therefore defines a morphism of cosimplicial $R$-modules

$$
A_{G} \otimes^{\Delta} A_{H} \rightarrow A_{G \times H} .
$$

This then defines a morphism

$$
\widetilde{A}_{G} \otimes \widetilde{A}_{H} \simeq\left(\widetilde{A_{G} \otimes A_{H}}\right) \rightarrow\left(\widetilde{A_{G} \otimes^{\Delta}} A_{H}\right) \rightarrow \widetilde{A_{G \times H}} .
$$

A.5. Now assume that both $M$ and $N$ are flat $R$-modules and that $R \Gamma(G, M)$ and $R \Gamma(H, N)$ lie in $D^{b}(R)$ (the bounded derived category of complexes of $R$ modules). Then $\widetilde{A}_{G}$ and $\widetilde{A}_{H}$ are also complexes of flat $R$-modules, so the map A.4.2 defines a morphism in $D(R)$

$$
R \Gamma(G, M) \otimes^{\mathbb{L}} R \Gamma(H, N) \rightarrow R \Gamma(G \times H, M \otimes N) .
$$

Theorem A.6 (Künneth formula). The map A.5.1 is an isomorphism.

Proof. It suffices to show that for any $[n] \in \Delta$, the map A.4.1 is an isomorphism.

For this note that the inclusion $G^{n} \hookrightarrow G^{[n]}$ sending

$$
\left(g_{1}, \ldots, g_{n}\right) \mapsto\left(1, g_{1}, \ldots, g_{n}\right) \in G^{[n]}
$$

defines an isomorphism

$$
\operatorname{Hom}_{G}^{\text {cts }}\left(G^{[n]}, M\right) \simeq \operatorname{Hom}^{\text {cts }}\left(G^{n}, M\right),
$$

and similarly for $H$ and $G \times H$. It therefore suffices to show that the natural map

$$
\operatorname{Hom}^{\text {cts }}\left(G^{n}, M\right) \otimes_{R} \operatorname{Hom}^{\text {cts }}\left(H^{n}, N\right) \rightarrow \operatorname{Hom}^{\text {cts }}\left((G \times H)^{n}, M \otimes N\right)
$$

is an isomorphism. Since $M$ (resp. $N$ ) is a continuous representation of $G$ (resp. $H$ ) we have

$$
M=\cup_{U} M^{U}, \quad\left(\text { resp. } N=\cup_{V} N^{V}\right),
$$

where $U$ (resp. $V$ ) runs over open normal subgroups of $G$ (resp. $H$ ). Since the formation of tensor product commutes with direct limits, it follows that we also have

$$
M \otimes N=\underset{(\overrightarrow{U, V})}{\lim _{(} M^{U} \otimes N^{V}}
$$

We therefore have

$$
\operatorname{Hom}^{\text {cts }}\left(G^{n}, M\right) \otimes \operatorname{Hom}^{\text {cts }}\left(H^{n}, N\right)=\underset{U, V}{\lim _{U}} \operatorname{Hom}^{\text {cts }}\left(G^{n}, M^{U}\right) \otimes \operatorname{Hom}^{\text {cts }}\left(H^{n}, N^{V}\right),
$$

and

$$
\operatorname{Hom}^{\operatorname{cts}}\left((G \times H)^{n}, M \otimes N\right)=\underset{U, V}{\lim _{U}} \operatorname{Hom}^{\operatorname{cts}}\left((G \times H)^{n}, M^{U} \otimes N^{V}\right) .
$$

It therefore suffices to prove that the map A.6.1 is an isomorphism in the case when $M=M^{U}$ and $N=N^{V}$ for some open normal subgroups $U \subset G$ and $V \subset H$. 
In this case, let $\left(U_{i}\right)_{i \in I}$ (resp. $\left.\left(V_{j}\right)_{j \in J}\right)$ be the set of open normal subgroups in $G$ (resp. $H$ ) contained in $U$ (resp. $V$ ). We then have

and

$$
\begin{aligned}
& \operatorname{Hom}^{\text {cts }}\left(G^{n}, M\right)=\underset{i}{\lim } \operatorname{Hom}\left(\left(G / U_{i}\right)^{n}, M\right), \\
& \operatorname{Hom}^{\text {cts }}\left(H^{n}, N\right)=\underset{j}{\lim } \operatorname{Hom}\left(\left(H / V_{j}\right)^{n}, N\right),
\end{aligned}
$$

$$
\operatorname{Hom}^{\text {cts }}\left((G \times H)^{n}, M \otimes N\right)=\underset{i, j}{\lim _{i, j}} \operatorname{Hom}^{\text {cts }}\left(\left(G / U_{i} \times H / V_{j}\right)^{n}, M \otimes N\right) .
$$

This reduces the proof to showing that the maps

$$
\operatorname{Hom}\left(\left(G / U_{i}\right)^{n}, M\right) \otimes \operatorname{Hom}\left(\left(H / V_{j}\right)^{n}, N\right) \rightarrow \operatorname{Hom}^{\text {cts }}\left(\left(G / U_{i} \times H / V_{j}\right)^{n}, M \otimes N\right)
$$

are isomorphisms, which is immediate.

COROllary A.7. Suppose in addition to the hypotheses of A.5 that the groups $H^{i}(G, M)$ and $H^{i}(H, N)$ are projective $R$-modules for all $i$. Then the natural map of graded $R$-modules

$$
H^{*}(G, M) \otimes_{R} H^{*}(H, N) \rightarrow H^{*}(G \times H, M \otimes N)
$$

is an isomorphism.

Proof. Since $R \Gamma(G, M)$ and $R \Gamma(H, N)$ are bounded complexes of $R$-modules with cohomology groups projective $R$-modules, we have isomorphisms in $D(R)$

$$
R \Gamma(G, M) \simeq H^{*}(G, M), \quad R \Gamma(H, N) \simeq H^{*}(H, N),
$$

where $H^{*}(G, M)$ and $H^{*}(H, N)$ are viewed as complexes with zero differentials. From A.6 we conclude that

$$
R \Gamma(G \times H, M \otimes N) \simeq H^{*}(G, M) \otimes_{R} H^{*}(H, N),
$$

and in particular for every $i$ the $R$-module $H^{i}(G \times H, M \otimes N)$ is a projective $R$-module. Therefore we also have

$$
R \Gamma(G \times H, M \otimes N) \simeq H^{*}(G \times H, M \otimes N)
$$

which implies the corollary.

\section{References}

[1] M. Artin, A. Grothendieck, and J.-L. Verdier, Théorie des topos et cohomologie étale des schémas, Lecture Notes in Mathematics 269, 270, 305, Springer-Verlag, Berlin (1972).

[2] L. Berger, An introduction to the theory of p-adic representations, in "Geometric aspects of Dwork theory", Walter de Gruyter, Berlin (2004), 255-292.

[3] _ Représentations p-adiques et équations différentielles, Invent. Math. 148 (2002), 219-284.

[4] P. Berthelot and A. Ogus, Notes on crystalline cohomology, Princeton U. Press, Princeton, 1978.

[5] C. Breuil, Integral p-adic Hodge theory, Adv. Stud. Pure Math., 36 (2002), 51-80.

[6] Sur quelques représentations modulaires et p-adiques de $\mathrm{GL}_{2}\left(\mathbf{Q}_{p}\right)$. I, Compositio Math. 138 (2003), 165-188.

[7] _ Sur quelques représentations modulaires et p-adiques de $\mathrm{GL}_{2}\left(\mathbf{Q}_{p}\right)$. II, J. Inst. Math. Jussieu 2 (2003), 23-58.

[8] C. Breuil and W. Messing, Torsion étale and crystalline cohomologies, Astérisque 279 (2002), 81-124.

[9] P. Deligne, Cohomologie étale, Lecture Notes in Mathematics 569 (1977).

[10] P. Deligne, Théorie de Hodge: II, Publ. Math. IHES 40 (1971), 5-57. 
[11] J. Dieudonné and A. Grothendieck, Éléments de géométrie algébrique, Inst. Hautes Études Sci. Publ. Math. 4, 8, 11, 17, 20, 24, 28, 32 (1961-1967).

[12] D. Eisenbud, Commutative algebra. With a view toward algebraic geometry, Graduate Texts in Mathematics 150, Springer-Verlag, New York, 1995.

[13] G. Faltings, Almost étale extensions, Astérisque 279 (2002), 185-270.

[14] _ Crystalline cohomology and p-adic Galois-representations, Algebraic analysis, geometry, and number theory (Baltimore, MD, 1988), Johns Hopkins Univ. Press, Baltimore, MD (1989), 191-224.

[15] _ P-adic Hodge theory, J. Amer. Math. Soc. 1 (1988), 255-299.

[16] J. M. Fontaine, Le Corps des Périodes p-adiques, Asterisque 223 (1994), 59-111.

[17] _ Représentations p-adique semi-stable, Asterisque 223 (1994), 113-184.

[18] J. M. Fontaine and W. Messing, $p$-adic periods and p-adic étale cohomology, in Contemporary Math 67 (1987), 179-207.

[19] W. Fulton, Intersection theory, Second edition. Ergebnisse der Mathematik und ihrer Grenzgebiete. 3. Folge. A Series of Modern Surveys in Mathematics, 2. Springer-Verlag, Berlin, 1998.

[20] H. Furusho, p-adic multiple zeta values. I. $p$-adic multiple polylogarithms and the $p$-adic KZ equation, Inven. Math. 155 (2004), 253-286.

[21] O. Gabber and L. Ramero, Almost ring theory, Lecture Notes in Math 1800, Springer-Verlag (2003).

[22] $\longrightarrow$ Foundations of p-adic Hodge theory, preprint (2005).

[23] P. Gabriel, Des catégories abéliennes, Bull. Soc. Math. France 90 (1962), 323-448.

[24] A. Grothendieck, Cohomologie l-adic et Fonctions L, Lectures Notes in Math 589 (1977).

[25] _ Revêtements étales et groupe fondamental, Lectures Notes in Math 224 (1971).

[26] O. Hyodo and K. Kato, Semi-stable reduction and crystalline cohomology with logarithmic poles, Asterisque 223 (1994), 221-268.

[27] L. Illusie, Crystalline cohomology, Proc. Sympos. Pure Math 55 (1994), 43-70.

[28] L. Illusie, Complexe cotangent et déformations. I., Lecture Notes in Mathematics 239, Springer-Verlag, Berlin-New York (1971).

[29] K. Kato, Logarithmic structures of Fontaine-Illusie, Algebraic analysis, geometry, and number theory (Baltimore, MD, 1988), Johns Hopkins Univ. Press, Baltimore, MD, 1989, pp. 191224.

[30] _ Semi-stable reduction and p-adic étale cohomology, Asterisque 223 (1994), 269-293.

[31] _ On p-adic vanishing cycles (Application of ideas of Fontaine-Messing), Advanced Studies in Pure Math 10 (1987), 207-251.

[32] K. Kato and W. Messing, Syntomic cohomology and p-adic étale cohomology, Tohoku Math. J. 44 (1992), 1-9.

[33] K. Kato and T. Saito, On the conductor formula of Bloch, Inst. Hautes Études Sci. Publ. Math. 100 (2004).

[34] K. Kedlaya, P-adic cohomology, this volume.

[35] M. Kim, The motivic fundamental group of the projective line minus three points and the theorem of Siegel, Invent. Math. 161 (2005), 629-656.

[36] W. Niziol, Crystalline conjecture via K-theory, Ann. Sci. École Norm. Sup. 31 (1998), 659681.

[37] _ Semistable conjecture via K-theory, Duke Math. J. 141 (2008), 151-178.

[38] A. Ogus, F-crystals, Griffiths transversality, and the Hodge decomposition, Astérisque 221 (1994).

[39] _ The convergent topos in characteristic p, The Grothendieck Festschrift, Vol. III, Progr. Math. 88 (1990), 133-162.

[40] _ F-isocrystals and de Rham cohomology II - Convergent Isocrystals, Duke Math Journal 51 (1984), 765-849.

[41] M. Olsson, Towards non-abelian P-adic Hodge theory in the good reduction case, preprint (2004).

[42] Sheaves on Artin stacks, J. Reine Angew. Math. (Crelle's Journal) 603 (2007), $55-112$.

[43] J.-P. Serre, Local fields, Graduate Texts in Mathematics 67, Springer-Verlag (1979).

[44] _ Galois cohomology, Springer-Verlag, Berlin (1997). 
[45] A. Shiho, Crystalline fundamental groups. I. Isocrystals on log crystalline site and log convergent site, J. Math. Sci. Univ. Tokyo 7 (2000), 509-656.

[46] Crystalline fundamental groups. II. Log convergent cohomology and rigid cohomology, J. Math. Sci. Univ. Tokyo 9 (2002), 1-163.

[47] J. Tate, p-divisible groups, in Proc. of a conference on local fields, NUFFIC Summer school, Driebergen, Springer (1967), 158-183.

[48] R. Thomason, Algebraic K-theory and étale cohomology, Ann. Sci. cole Norm. Sup. 18 (1985)437-552.

[49] T. Tsuji, p-adic étale cohomology and crystalline cohomology in the semi-stable reduction case, Inv. Math. 137 (1999), 233-411.

[50] _ Crystalline sheaves, syntomic cohomology, and p-adic polylogarithms, notes from a seminar at Cal Tech on Feb. 20, 2001.

[51] _ Semi-stable conjecture of Fontaine-Jannsen: a survey. Cohomologies p-adiques et applications arithmétiques, II, Astérisque 279 (2002), 323-370.

[52] V. Vologodsky, Hodge structure on the fundamental group and its application to p-adic integration, Mosc. Math. J. 3 (2003), 205-247.

[53] C. Weibel, An introduction to homological algebra, Cambridge Univ. Press (1994).

Department of Mathematics, University of California, Berkeley, CA 94720-3840 USA

E-mail address: molsson@math.berkeley.edu 